\title{
Certified Reduced Basis Methods for PARAmetrized SAdDle Point Problems
}

Von der Fakultät für Mathematik, Informatik und Naturwissenschaften der RWTH Aachen University zur Erlangung des akademischen Grades einer Doktorin der Naturwissenschaften genehmigte Dissertation

\author{
vorgelegt von \\ Diplom-Mathematikerin \\ Anna-Lena Gerner \\ aus Hannover
}

Berichter: Jun.-Prof. Karen Veroy-Grepl, Ph.D.

Univ.-Prof. Dr. Arnold Reusken

Tag der mündlichen Prüfung: 16. Oktober 2012

Diese Dissertation ist auf den Internetseiten der Hochschulbibliothek online verfügbar. 

To my parents. 



\section{Abstract}

In this thesis, we present a new reduced basis approach to parametrized saddle point problems. The proposed methods allow us to consider flow problems in parametrized domains with notably greater ease.

Designed for the real-time and many-query context of parameter estimation, optimization, and control, the reduced basis method permits the efficient yet reliable approximation of input-output relationships induced by parametrized partial differential equations. The essential ingredients are: (i) dimension reduction, through Galerkin projection onto a lowdimensional reduced basis space; (ii) certainty, through rigorous a posteriori bounds for the errors in the reduced basis approximations; (iii) computational efficiency, through an Offline-Online computational strategy; and (iv) effectiveness, through a greedy sampling approach. Although reduced basis methods are well developed for several classes of partial differential equations, parametrized saddle point problems pose additional difficulties that have not been fully addressed: Parameter-dependent constraints cause complications not only in the choice of stable reduced basis approximation spaces, but also in the construction of rigorous and computationally efficient a posteriori error bounds.

Based on an analysis of Brezzi's theory for saddle point problems, this work extends and improves existing reduced basis approaches with respect to both the construction of effective reduced basis approximation spaces as well as a posteriori error bounds. This is achieved as follows: First, we develop new rigorous a posteriori error bounds, which perform better in terms of sharpness as well as computational efficiency. Second, we present and analyze several options to construct the reduced basis approximation spaces. Stability is achieved through enriching the reduced basis approximation space for the primal system variable appropriately; different strategies may be applied, favoring either the approximations for the primal or the Lagrange multiplier variables. Third, both a posteriori error bounds and enrichment strategies are employed in a new adaptive sampling procedure for constructing reduced basis approximation spaces that are not only stable but also efficient.

Motivated by applications in the field of microfluidics, the methods are applied to a Stokes flow in a parametrized domain. Providing a direct comparison with former reduced basis techniques, numerical results demonstrate the methods' successful performance in practice. 



\section{Zusammenfassung}

In der vorliegenden Arbeit präsentieren wir einen neuen Zugang zu Reduzierte-Basis-Methoden für parametrisierte Sattelpunktprobleme. Die daraus resultierenden numerischen Verfahren vereinfachen die Analyse von Strömungsproblemen in parameterabhängigen Gebieten erheblich.

Reduzierte-Basis-Methoden ermöglichen eine effiziente und zuverlässige Approximierung von Input-Output Beziehungen, die durch parametrisierte partielle Differentialgleichungen beschrieben werden. Die wesentlichen Bestandteile sind die Folgenden: (i) Dimensionsreduktion, durch Galerkin Projektion auf einen niedrigdimensionalen Approximationsraum; (ii) Gewissheit, durch a posteriori Fehlerschranken; (iii) Effizienz, durch eine Offline-Online Rechenstrategie; und (iv) Effektivität, durch ein Greedy-Sampling-Verfahren. ReduzierteBasis-Methoden sind gut entwickelt für bestimmte Klassen von partiellen Differentialgleichungen. Parametrisierte Sattelpunktprobleme jedoch stellen uns vor zusätzliche Herausforderungen, die noch nicht vollständig behandelt wurden: Parameterabhängige Nebenbedingungen verursachen Komplikationen nicht nur in der Konstruktion von stabilen Approximationsräumen, sondern auch in der Entwicklung effizienter a posteriori Fehlerschätzer.

Diese Arbeit erweitert und verbessert bestehende Ansätze basierend auf einer Analyse der Sattelpunkttheorie von F. Brezzi. Dies erreichen wir wie folgt: Erstens entwickeln wir neue $a$ posteriori Fehlerschätzer, die sowohl effizienter als auch schärfer sind als bisherige Schranken. Zweitens präsentieren und untersuchen wir verschiedene Optionen zur Konstruktion des reduzierten Approximationsraums. Stabilität wird erzielt durch eine geeignete Anreicherung des Approximationsraums für die primale Systemvariable; verschiedene Strategien stehen hier zur Auswahl, die sich entweder auf die Approximation der primalen Systemvariable oder die des Lagrange-Multiplikators konzentrieren. Drittens stellen wir ein neues adaptives Sampling-Verfahren vor, das angepasst ist an die Bedürfnisse von Sattelpunktproblemen. Die entwickelten Fehlerschätzer und Stabilisierungstechniken werden hier zielführend eingesetzt zur Konstruktion von Approximationsräumen, die nicht nur stabil, sondern auch effizient sind.

Die hergeleiteten Methoden werden angewendet auf ein Stokes Strömungsproblem, welches motiviert ist durch Problemstellungen im Bereich der Mikrofluidik. Numerische Ergebnisse demonstrieren die Leistungsfähigkeit der entwickelten Verfahren durch einen direkten Vergleich mit derzeit bestehenden Techniken. 



\section{Acknowledgments}

I have had the most wonderful fortune of having Professor Karen Veroy-Grepl as my supervisor. To her, I would like to express my deep gratitude. I am most thankful not only for her extraordinary support, guidance, and counsel during the past three years, but also for her patience, humor, passion, and trust. Despite her full schedule, she would be approachable at any time to share her knowledge; she would always have a useful suggestion of how to tackle a specific issue, check my notes very carefully to point out difficulties or imprecisions, and provide detailed feedback on my presentations.

I further enjoyed the great fortune of having Professor Arnold Reusken as my second advisor. I would like to thank him for many invaluable comments and suggestions as well as for his encouragement and patience. Even at short notice, he would find the time to read my work or discuss my questions. Often confronted with a particular problem that could not yet be formulated very clearly, he would find the words that I was looking for.

I would also like to thank the members of my thesis committee, Professor Wolfgang Dahmen and Professor Josef Bemelmans, for their comments, suggestions, as well as their time for patiently answering my questions.

During my doctoral studies, I have had the opportunity to highly benefit from the friendly and inspiring atmosphere within the reduced basis community. I am truly grateful to Professor Anthony T. Patera for having me at MIT and for his constant support and counsel throughout my studies. I would like to thank Professor Stefan Volkwein for inviting me to the University of Konstanz, where I felt very welcome and enjoyed numerous interesting and helpful discussions. I am also very grateful to Professor Martin A. Grepl of RWTH Aachen University and Professor Yvon Maday of Laboratoire Jacques-Louis Lions for their comments, suggestions, and thought-provoking questions. For the numerical results presented in this thesis, I extensively used open source software. I would like to thank Dr. David J. Knezevic of Harvard University for his invaluable assistance and support on rb00mit [65], providing excellent help typically within minutes. I also very much appreciate the dependable support of the developers of libMesh [62].

I would like to thank my fellow colleagues at $\mathrm{AICES}^{1}$ for the open, supportive, and encouraging atmosphere, the AICES service team for their support on administrative and organizational tasks, as well as Professor Marek Behr and Dr. Nicole Faber for leading AICES in such a dedicated way. I am particularly grateful to my current and former office mates: Markus Bachmayr, for many helpful discussions off and on the topic as well as for an excellent teamwork while being student representatives; Lorenzo Zanon, for sharing

\footnotetext{
${ }^{1}$ Aachen Institute for Advanced Study in Computational Engineering Science http://www. aices.rwth-aachen.de
} 
numerous computational difficulties and bearing my grim debugging-face without complaint; and Mark Kärcher, for often being concerned with similar questions, for many valuable discussions and comments, as well as for proof-reading parts of this thesis very carefully.

I would like to thank Kathrin Knappmann for finding me right at the beginning of the first semester, studying with me in what I call a most fruitful long-term assignment-partnership, and staying in my life ever since as my close friend. I am truly grateful to Jan Hendrik Witte for his exceptional patience and confidence, and particularly, for believing in me and being my soul mate in many nerdy ways.

Finally, I would like to express my deepest gratitude and love to my family. I would like to thank my brother Martin, my sister-in-law Kattrin, and my two lovable little nephews, Anton and Lars, for their continuous support and care. I would like to thank my sister and best friend, Julia, for joining me in Aachen as well as for numerous home made cakes and cookies, but most of all, for her generousness, trust, tolerance, and understanding.

Above all, I would like to thank my parents, Claudia and Helmut Gerner, for their infinite patience, understanding, and love. Without their support, I would never have been able to pursue my dreams. 


\section{Contents}

1 Introduction 1

1.1 Motivation . . . . . . . . . . . . . . . . . . . . . 1

1.2 Model Order Reduction . . . . . . . . . . . . . . . . . . . . . . . . . 1

1.3 The Reduced Basis Method . . . . . . . . . . . . . . . . . . . . 3

1.4 Contribution of this Thesis . . . . . . . . . . . . . . . 5

1.4.1 Theory - Parametrized Saddle Point Problems . . . . . . . . . . . . 5

1.4.2 Application — Incompressible Fluid Flow . . . . . . . . . . . . 5

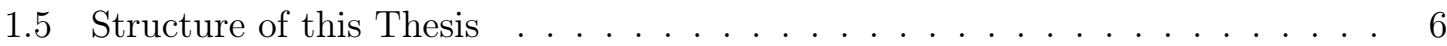

2 Approximation of Saddle Point Problems 9

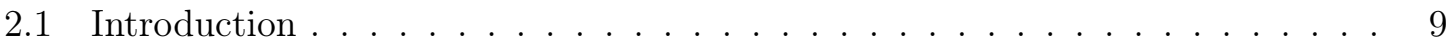

2.2 General Problem Statement . . . . . . . . . . . . . . . . . . . . . . . . . 10

2.2 .1 Formulation . . . . . . . . . . . . . . . . . . . . 10

2.2.2 Truth Approximation . . . . . . . . . . . . . . . . . 11

2.3 Reduced Basis Approximation . . . . . . . . . . . . . . . . . . . . 12

2.3 .1 Formulation . . . . . . . . . . . . . . . . . . . 12

2.3.2 Properties of the Discrete System . . . . . . . . . . . . . . . . 13

2.3.3 A Priori Error Estimation . . . . . . . . . . . . . . . . . . . . . 13

2.4 A Posteriori Error Estimation . . . . . . . . . . . . . . . . . . . . . . 16

2.4 .1 Ingredients . . . . . . . . . . . . . . . . 16

2.4.2 Error Bound Formulation . . . . . . . . . . . . . . . . . 16

2.5 Construction of Reduced Basis Approximation Spaces . . . . . . . . . . . . 23

2.6 Offline-Online Computational Procedure . . . . . . . . . . . . . . . . . 25

2.6.1 Reduced Basis Approximation . . . . . . . . . . . . . . . . . . . 25

2.6.2 A Posteriori Error Bounds . . . . . . . . . . . . . . . . . . . . . 26

2.7 Adaptive Sampling Procedures . . . . . . . . . . . . . . . . . . . . . 31

2.8 Concluding Remarks . . . . . . . . . . . . . . . . . . . . . . . . . 34

3 Approximation of the Stokes Equations $\quad 37$

3.1 Introduction . . . . . . . . . . . . . . . . . . 37

3.2 Model Problem . . . . . . . . . . . . . . . . . . . . . 37

3.2 .1 Strong Formulation . . . . . . . . . . . . . . . . . . . 38

3.2 .2 Weak Formulation . . . . . . . . . . . . . . . . . . . . 39

3.2.3 Formulation as a Parametrized Saddle Point Problem . . . . . . . . . 39 
3.3 Numerical Results . . . . . . . . . . . . . . . . . . . . . . . . . . . 41

3.3.1 Truth Approximation . . . . . . . . . . . . . . . . . 44

3.3.2 Reduced Basis Approximation Spaces . . . . . . . . . . . . . . . . 42

3.3.3 Adaptive Sampling Procedures . . . . . . . . . . . . . . . . . . 45

3.3.4 A Posteriori Error Bounds . . . . . . . . . . . . . . . . . . . . . 47

3.3.5 Computation Times . . . . . . . . . . . . . . . . . . 53

3.4 Concluding Remarks . . . . . . . . . . . . . . . . . . . . . . 54

4 Approximation of Saddle Point Problems by Penalty $\mathbf{5 5}$

4.1 Introduction . . . . . . . . . . . . . . . . . . . . . . . . 55

4.2 General Problem Statement . . . . . . . . . . . . . . . . 56

4.2 .1 Formulation . . . . . . . . . . . . . . . 56

4.2.2 Truth Approximation . . . . . . . . . . . . . . . . . 58

4.3 Reduced Basis Approximation . . . . . . . . . . . . . . . . . . . . 59

4.3 .1 Formulation . . . . . . . . . . . . . . . . . 60

4.3.2 Properties of the Discrete System . . . . . . . . . . . . . . . 60

4.3.3 A Priori Error Estimation . . . . . . . . . . . . . . . . . . . 60

4.4 A Posteriori Error Estimation . . . . . . . . . . . . . . . . . . . . 64

4.4 Ingredients . . . . . . . . . . . . . . . . . 64

4.4 Error Bound Formulation . . . . . . . . . . . . . . . . . . 64

4.5 Construction of Reduced Basis Approximation Spaces . . . . . . . . . . . 67

4.6 Offline-Online Computational Procedure . . . . . . . . . . . . . . 67

4.6.1 Reduced Basis Approximation . . . . . . . . . . . . . . . . 67

4.6.2 A Posteriori Error Bounds . . . . . . . . . . . . . . . . . . 68

4.7 Adaptive Sampling Procedures . . . . . . . . . . . . . . . . . . . 69

4.8 Concluding Remarks . . . . . . . . . . . . . . . . . . . . . . 70

5 Approximation of the Stokes Equations by Penalty $\quad 73$

5.1 Introduction . . . . . . . . . . . . . . . . . . 73

5.2 Model Problem . . . . . . . . . . . . . . . . . . . . . . . 73

5.3 Numerical Results . . . . . . . . . . . . . . . . . . . . . . . . 74

5.3 .1 Truth Approximation . . . . . . . . . . . . . . . . . . 74

5.3.2 Reduced Basis Approximation Spaces . . . . . . . . . . . . . . 76

5.3.3 A Posteriori Error Bounds . . . . . . . . . . . . . . . . . . . . 82

5.3 .4 Computation Times . . . . . . . . . . . . . . . . . 8 87

5.4 Concluding Remarks . . . . . . . . . . . . . . . . . . . . . . . 88

6 Approximation of the Instationary Stokes Equations $\quad 91$

6.1 Introduction . . . . . . . . . . . . . . . . . . . . . . . 91

6.2 General Problem Statement . . . . . . . . . . . . . . . . . . 92

6.2 .1 Formulation . . . . . . . . . . . . . . . . . 92

6.2.2 Truth Approximation . . . . . . . . . . . . . . . . . 93

6.3 Reduced Basis Approximation . . . . . . . . . . . . . . . . . . . . 94

6.4 A Posteriori Error Estimation . . . . . . . . . . . . . . . . . . . 94

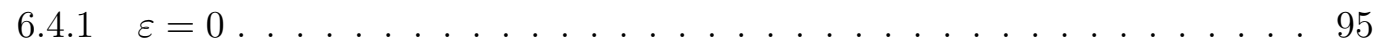




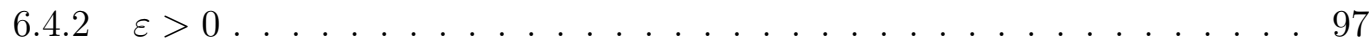

6.5 Offline-Online Computational Procedure . . . . . . . . . . . . . . . . . . 99

6.6 Construction of Reduced Basis Approximation Spaces . . . . . . . . . . . . . 99

6.7 Model Problem . . . . . . . . . . . . . . . . . . . . . . . . . . . . . . . . . . . 102

6.8 Numerical Results . . . . . . . . . . . . . . . . . . . . . . . . . . . . . . . 103

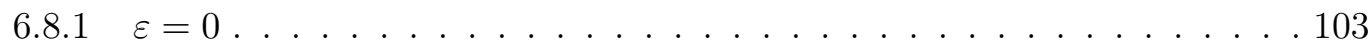

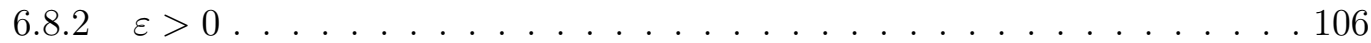

6.9 Concluding Remarks . . . . . . . . . . . . . . . . . . . . . . 111

$\begin{array}{ll}\text { References } & 112\end{array}$

$\begin{array}{ll}\text { A Notations } & 121\end{array}$

B Supplementary Comments 123

C Re: Chapter 6 - A Crank-Nicolson Method 125 



\section{Chapter 1}

\section{Introduction}

\subsection{Motivation}

Despite impressive progress in mechanical modeling, numerical analysis, and computer science during the last decades, many engineering problems remain intractable. With the increase in speed and memory capacity of computing devices and the improvement of discretization techniques, also new challenges emerged. Models describing physical problems have to face new requirements in terms of accuracy or real-time applicability, resulting in a numerical complexity that is prohibitive for current technologies.

In many engineering problems, the aim is to optimize, control, or characterize a system whose behavior is governed by a partial differential equation (PDE). Such an analysis often requires the prediction of outputs in dependence of inputs: Outputs are expressed as functionals evaluated at the solution of the governing PDE, whereas inputs are parameters that define a particular system configuration and enter through the PDE. Outputs of interest may be given by a temperature distribution, structural frequencies, or a channel flowrate; inputs often describe material properties, loads and sources, or geometry. For the efficient numerical simulation of such input-output relationships, classical discretization techniques such as finite differences, finite volume, or finite element methods are generally too expensive. First, the context of process control, parametric modeling, or shape optimization often requires the repeated output prediction for many different input parameter values. Second, numerous branches of science and engineering call for an accurate and reliable response in real time. Third, light computing devices such as smartphones or tablets, which may be employed working in the field but are equipped with less computing power, become appealing alternatives to heavy computing platforms. At the present time, these problems cannot be tackled in a direct, traditional way. The reduced basis method, a model order reduction technique, is one means to address this difficulty.

\subsection{Model Order Reduction}

Reduced order modeling has received considerable attention as it can often capture the system behavior at significantly less cost. The idea is to replace the original model by an approximate model that is much smaller in terms of dimension but still describes important aspects of the system. This involves a number of challenging issues such as (i) the identi- 
fication of crucial system properties that should be preserved by the reduced order model, (ii) the extraction of relevant data from the original model that is needed to generate the reduced order model, (iii) the numerically stable computation of the reduced order model, and (iv) the characterization and quantification of its quality. With its own focus and special needs, every discipline tackles these issues differently; as a result, there exist many model order reduction approaches. Among the most well-known techniques for dynamical systems are proper orthogonal decomposition (POD), balanced truncation, and Krylov subspace methods. For a comprehensive overview of these methods, we refer the reader to, e.g., $[3,8,21,30,43,90]$ and the references therein; we shall here only summarize the main ideas that allow us to distinguish the reduced basis method.

One of the central issues of POD is the reduction of data expressing their essential information by means of a few basis vectors (see, e.g., [67, 93]). Assume that we are given a set of data in a high-dimensional space, extracted from the original system by either precomputations or experiments. The idea is then to find a subspace of a fixed dimension $r$ such that the error in the projection onto the subspace is minimized. This is achieved by applying the singular value decomposition (SVD) to the data matrix: The optimal subspace is obtained as the span of the $r$ orthonormalized eigenvectors associated with the largest singular values; these vectors are also called the POD modes.

Balanced truncation and Krylov subspace methods aim to reduce the order of a dynamical system such that the implied input-output relationship is well approximated. The latter is characterized by an input-output map, the so-called transfer function. The objective is then to construct a reduced order model with a transfer function resembling the original one as far as possible.

To this end, balanced truncation (see, e.g., $[4,8,90]$ ) proceeds as follows: As the name suggests, the system is first balanced and then truncated. Balancing a dynamical system refers to identifying which states of the system are important to its input-output map. To decide whether it affects the system's input-output map, a state is analyzed with respect to its controllability and observability: Roughly speaking, a state is controllable if it can be reached through an input; a state is observable if it noticeably contributes to an output. Only the states that are both controllable and observable are of relevance. A useful measure of a state's controllability and observability is given by the controllability and observability gramians. A dynamical system is called balanced if these two gramians coincide and equal a diagonal matrix containing the so-called Hankel Singular Values (HSVs): In this case, states that are difficult to control are simultaneously difficult to observe; quantifying this behavior, the HSVs provide an order of relevance to the input-output map. We may then truncate the state space dimensions with low HSVs to obtain a lower-dimensional, approximate model.

Krylov subspace methods (see, e.g., [6, 30]) do not rely on the computation of singular values but are based on moment matching. Noting that the transfer function of a dynamical system can be expanded into a Laurent series, they assume that essential properties of the input-output relationship are reflected in the associated coefficients; these coefficients are also called moments. Given a fixed dimension $r$, the reduced model of corresponding order is therefore constructed by matching as many moments as possible. Since computing the moments explicitly is inherently numerically unstable, only the observation that moment matching is equivalent to projecting the state space onto suitable Krylov subspaces made the approach feasible in practice. 
We emphasize that, even though coming from various fields with different objectives, the above model order reduction techniques share a common basic principle: They all can be interpreted as Galerkin or Petrov-Galerkin type projections of the original space onto a low-dimensional subspace. However, different from the others in its particular choice of this subspace (either given through the span of the POD modes, the range of the controllability and observability gramians, or a Krylov subspace), each method has its own benefits and limitations. Based on a very general concept, POD is applicable to various fields and has been successfully used in, e.g., fluid dynamics, signal analysis, data compression, and control theory (see, e.g., $[21,67,69]$ and the references therein); depending on the respective context, it is also known as principal component analysis or the Karhunen-Loève decomposition. The method can be applied to high-complexity linear as well as nonlinear systems (see [3]) and is equipped with useful error estimators (see, e.g., [107] and the references therein). However, POD can yield unpredictable results as it is sensitive to the data being used and important system properties such as stability or passivity are generally not preserved; even though the POD modes are optimal at approximating a given data set, they are not necessarily optimal for describing the dynamics of the system that generates this data (see [93]). Designed for the specific needs in optimal control, balanced truncation preserves system properties such as stability and passivity (see $[8,43])$. But, to obtain the required gramians and HSVs, it involves the computationally highly expensive solution of two Lyapunov equations followed by an SVD, and is therefore hardly suitable for large-scale problems (see [4, 93]). The latter can be handled efficiently by Krylov subspace methods (see [6, 30]); however, those often provide a good approximation quality only locally and lack the availability of useful error estimates (see $[3,8]$ ).

To overcome each others limitations, the methods are often combined; see, for example, $[93,108]$ for approaches combining POD and balanced truncation, and [3] for methods that aim to team the best features of SVN based and Krylov subspace methods.

\subsection{The Reduced Basis Method}

The reduced basis method is a model order reduction approach that permits the efficient yet reliable approximation of input-output relationships induced by parametrized partial differential equations. In contrast to generic discretization techniques where approximation spaces are not correlated to the physical properties of the system, the method recognizes that the solutions to a parametrized PDE are not arbitrary members of the infinite-dimensional solution space but rather reside or evolve on a much lower-dimensional manifold. Exploitation of this low-dimensionality is the key idea of the reduced basis approach.

Designed for the real-time and many-query context of parameter estimation, optimization, and control, the method provides rapidly convergent and computationally efficient approximations equipped with practicable and rigorous error bounds. In compliance with model order reduction techniques outlined in $\S 1.2$, this is achieved via Galerkin projection: Built upon a high-fidelity "truth" finite element discretization, the reduced basis approximation is defined as a Galerkin projection onto a low-dimensional subspace that focuses on the solution manifold induced by the parametrized PDE; following the so-called Lagrange approach, this subspace is constructed as the span of several "truth" solutions of the problem at various parameter values. The error in the reduced basis approximation is then measured 
relative to the "truth" problem formulation and can be quantified by rigorous a posteriori error bounds; the latter are typically derived as relaxations of the error-residual equation or can be obtained through direct application of appropriate a priori stability estimates. To the method's key features also belongs an Offline-Online computational strategy that enables the highly efficient (Online) computation of both reduced basis approximations and error bounds for any parameter query at the expense of increased pre-processing (Offline) cost. Finally, reduced basis approximations and error bounds are intimately linked through a greedy sampling approach, in which the (Online-)inexpensive error bounds are used to construct the reduced basis approximation spaces more optimally.

A detailed review of the reduced basis method, including its historical background and further references, can be found in [83,95]; here, we shall restrict ourselves to the stages that help us embed this thesis in its natural context. The method was introduced in the late 1970s as a successful tool for describing large systems in nonlinear structural mechanics with only a few basis functions (see, e.g., $[2,28,29,80,85,86]$ ). Thenceforth, literature grew rapidly; it soon extended the approach to a wider class of applications and equations, such as fluid dynamics and the incompressible Navier-Stokes equations (see, e.g., [45, 56, 57, 58, 84]). However, the full potential of the method was not recognized until work in [71, 73, 87] entirely decoupled the reduced basis model generation from the projection stage and introduced the concept of computationally inexpensive a posteriori error estimators. Offline-Online decomposition strategies gained computational savings of several orders of magnitude. A posteriori error bounds are clearly crucial to the certification of any particular output prediction, but also play an important role in the effective and efficient model generation: Invoked in a greedy sampling process, they allow us to determine (i) which basis functions might be most effective and (ii) how many basis functions are needed to guarantee a desired accuracy. Much current effort is therefore devoted to the development of computationally inexpensive rigorous a posteriori error bounds [53, 55] and optimal sampling procedures [10, 15, 46].

Relevant to many applications such as heat conduction and linear elasticity in transport and continuum mechanics, work in [41, 73, 87, 95] established the reduced basis method for linear elliptic and parabolic PDEs with an affine parameter dependence. Henceforth, the method has been extended in various directions: Much of recent research focuses on its expansion to noncoercive as well as nonlinear equations in fluid dynamics, including the viscous Burgers' [78, 105, 110], the Stokes [92, 96], and the incompressible Navier-Stokes equations $[25,63,76,88,104]$. However, it has also been successfully applied to problems in acoustics [106], electromagnetics [22, 60], and stochastics [11, 49]. An empirical interpolation method (EIM) [7, 19, 26, 38, 40, 94] makes the approach feasible for PDEs with a nonaffine parameter dependence; methods in [54, 70, 74] handle hierarchical systems by combining reduced basis with domain decomposition techniques; and work in $[24,39,61,77,103]$ provides a reduced basis framework for the efficient solution of parametrized optimal control problems.

For the modeling of time-dependent parametrized PDEs, reduced basis methods are often combined with POD techniques (see §1.2). POD based schemes can be applied within the parametric reduced basis context [16, 44]; however, most approaches apply reduced basis greedy sampling strategies in the (multi-dimensional) parameter domain while using POD in the (one-dimensional) temporal domain (see, e.g., [41, 47, 63]). We note that also balanced truncation and Krylov subspace methods (see $§ 1.2$ ) have recently been extended 
to parameter-dependent systems; however, many of them in their beginnings, the proposed methods often lack practicable error estimates or can handle only very few parameters (see, e.g., $[17,59]$ and the references therein).

\subsection{Contribution of this Thesis}

In this thesis, we present a new reduced basis approach to parametrized saddle point problems. The proposed methods allow us to consider flow problems in parametrized domains with notably greater ease.

\subsubsection{Theory — Parametrized Saddle Point Problems}

Saddle point problems often arise in practical applications where a certain quantity has to be minimized subject to a set of linear constraints; examples of such applications are mixed finite element methods (see, e.g., $[13,89]$ ) or quadratic programming methods in optimal control (see, e.g., [66] and the references therein). In many cases, not only the primal system variable $u$ but also the Lagrange multiplier $p$ associated with the constraints has a physical interpretation and its computation is also of interest.

Although the reduced basis method is well developed for several classes of partial differential equations (see §1.3), parametrized saddle point problems pose additional difficulties that have not been fully addressed: Parameter-dependent constraints cause complications not only in the choice of stable reduced basis approximation spaces, but also in the construction of rigorous and computationally efficient a posteriori error bounds. Currently, stable reduced basis approximations are achieved through an enrichment of the approximation space for $u$ by so-called supremizer functions [72, 92, 96]; resulting in a reduced basis approximation space for $u$ that is at least twice as large as for $p$, this is not yet satisfactory. Error estimators are often based on Babuška's theory for noncoercive problems [75, 106]; this provides rigorous upper bounds for the combined error in the reduced basis approximations for $u$ and $p$, and requires the expensive computation of lower bounds to the Babuška inf-sup stability constants $[53,55]$.

In this work, we extend and improve existing reduced basis approaches for parametrized saddle point problems with respect to both the construction of effective reduced basis approximation spaces as well as a posteriori error bounds. This is achieved as follows: First, we develop new rigorous a posteriori error bounds that do not involve the estimation of the Babuška inf-sup stability constants but only much less expensive calculations. Second, separate upper bounds for the errors in the approximations for $u$ and $p$ enable the systematic estimation of engineering outputs depending on either of the two. Third, we introduce a new option to construct stable reduced basis approximation spaces that proves to be very effective. Fourth, recognizing the need for stabilization adaptively, a new sampling procedure assembles reduced basis approximation spaces that are very efficient.

\subsubsection{Application — Incompressible Fluid Flow}

Our focus shall be on saddle point problems that appear in the modeling of incompressible fluid flow. The incompressible Navier-Stokes equations are the governing equations to 
describe the motion of a viscous fluid (see, e.g., [36, 89]). They generally consist of two equations, the momentum and the continuity equations, which are derived from the basic principles of conservation of momentum and mass in classical mechanics; the unknowns are the velocity field $u$ of the fluid and the pressure $p$. In case of a creeping flow, where inertial forces are assumed to be small compared to viscous forces, the nonlinear convection term in the Navier-Stokes equations may be neglected and the equations reduce to the linear Stokes equations.

Quite often, it is crucial to analyze and understand the effects of geometric parameters on a flow, in particular when the objective is to optimize, control, or characterize the system. The reduced basis method allows us to perform such an analysis very efficiently. Earlier work on the Stokes and incompressible Navier-Stokes equations has established the method for nonparametrized domains [63, 104], and there have been several efforts [25, 54, 70, 76, 88, 92, 96] at extending the techniques to parametrized domains. However, due to the difficulties explained above (see §1.4.1), rigorous error bounds are often not treated and the geometric variations considered are very small or applicable only to a very limited set of problems.

Motivated by applications in the field of microfluidics, we apply our new methods to a Stokes flow in a two-dimensional microchannel with a parametrized, rectangular obstacle. Providing a direct comparison with former reduced basis techniques, numerical results demonstrate the proposed methods' successful performance in practice.

\subsection{Structure of this Thesis}

In Chapter 2, we present the abstract framework of a new reduced basis approach based on Brezzi's theory for saddle point problems. We present new a posteriori error estimates that provide rigorous upper bounds for the errors in the approximation of the primal system variable $u$ and the Lagrange multiplier $p$ separately; symmetric saddle point problems shall be treated as a special case in which these bounds may be further sharpened. Moreover, we analyze several options to construct the reduced basis approximation spaces with respect to approximation stability as well as their effectiveness. Finally, we present a new adaptive sampling procedure serving the needs of our saddle point context.

In Chapter 3, we apply the reduced basis approach developed in $\S 2$ to the Stokes equations in a parametrized domain. We consider a Stokes flow in a two-dimensional microchannel with a parametrized, rectangular obstacle; in the field of microfluidics, such microchannels are used to enhance the mixing between two adjacent laminar streams. Numerical results demonstrate the method's performance in practice through a direct comparison with former techniques: Reduced basis a posteriori error bounds are compared with respect to sharpness as well as computational cost; reduced basis approximation spaces are compared with respect to stability, effectiveness, and computational efficiency.

Chapter 2 and Chapter 3 are based on our work in [31, 33].

In Chapter 4 , we present a reduced basis method based on a penalty approach. The analysis shows how the introduction of a penalty term affects the development of reduced basis a posteriori error bounds as well as the construction of reduced basis approximation spaces. At the expense of a perturbed "truth" approximation, we may develop rigorous a posteriori error bounds that are computationally highly efficient as they do not depend on inf-sup stability constants; however, they depend on the penalty parameter, and associated effectivities 
increase as we approach the nonperturbed problem. Furthermore, the penalty term regularizes the problem such that an additional enrichment of the reduced basis approximation space for $u$ is no longer compulsory to obtain stable approximations.

In Chapter 5, we apply the reduced basis approach developed in $\S 4$ to a perturbed Stokes flow in a parametrized domain. Considering the model problem introduced in $§ 3$, numerical results demonstrate the effects of the penalty term in practice.

Chapter 4 and Chapter 5 are based on our work in [35].

In Chapter 6, we provide an outlook on how the techniques presented in the previous part of this thesis may be extended to the time-dependent setting. To this end, we consider the instationary Stokes equations. The approach essentially combines techniques developed in $\S 2, \S 3$ and $\S 4, \S 5$ with current reduced basis techniques for parabolic problems. The analysis then shows how time integration affects the development of reduced basis a posteriori error bounds as well as the construction of efficient reduced basis approximation spaces. The resulting methods are applied to a Stokes flow in a parametrized microchannel where evolution in time is induced by a time-dependent velocity profile on the inflow boundary. Numerical results focus on differences to the stationary case when demonstrating the method's performance in practice. The chapter is based on our work in [32, 34].

Note that each chapter opens with its own introduction, where we outline the chapter's purpose and content as well as its structure; each chapter closes with its own conclusion, where we emphasize particular benefits and limitations of the approach presented. 



\section{Chapter 2}

\section{Approximation of Saddle Point Problems}

\subsection{Introduction}

In this chapter, we present the abstract framework of a new reduced basis approach based on Brezzi's theory for saddle point problems. We shall focus on two important aspects: First, we present new a posteriori error estimates that, unlike earlier approaches based on Babuška's theory for noncoercive problems, provide upper bounds for the errors in the approximations for the primal system variable and the Lagrange multiplier separately. The proposed method is a direct application of techniques in $[12,13]$ to the reduced basis context (see [111] for initial investigations), which shall be further improved in the special case of a symmetric problem. Second, based on an analysis of Brezzi's theory, we present several options to construct the reduced basis approximation spaces that shall be compared with respect to approximation stability as well as their effectiveness. We achieve stability through enriching the reduced basis approximation space for the primal variable appropriately. Different strategies may be applied, favoring either the approximations for the primal or the Lagrange multiplier variables. Finally, both a posteriori error bounds and enrichment strategies are employed in an adaptive sampling procedure for constructing reduced basis approximation spaces that are not only stable but also computationally efficient.

The chapter is organized as follows: In $\$ 2.2$, we introduce the general formulation of a parametrized saddle point problem and its "truth" approximation upon which we shall build our reduced basis approximation. Section 2.3 defines our reduced basis approximation as the Galerkin projection onto a low-dimensional reduced basis approximation space; we shall recall main properties of the reduced basis system as well as a priori convergence results. We develop rigorous reduced basis a posteriori error bounds in $\S 2.4$, and discuss how to construct the reduced basis approximation space in $\S 2.5$; computational efficiency is achieved by the Offline-Online strategy summarized in $\S 2.6$. In $\S 2.7$, a new adaptive sampling procedure then combines error bounds derived in $\$ 2.4$ and observations in $\S 2.5$ to identify reduced basis approximation spaces that are stable as well as efficient. Finally, in $\S 2.8$, we give some concluding remarks. 


\subsection{General Problem Statement}

\subsubsection{Formulation}

Let $X_{\mathrm{e}}$ and $Y_{\mathrm{e}}$ be two Hilbert spaces with inner products $(\cdot, \cdot)_{X_{\mathrm{e}}},(\cdot, \cdot)_{Y_{\mathrm{e}}}$ and associated norms $\|\cdot\|_{X_{\mathrm{e}}}=\sqrt{(\cdot, \cdot)_{X_{\mathrm{e}}}},\|\cdot\|_{Y_{\mathrm{e}}}=\sqrt{(\cdot, \cdot)_{Y_{\mathrm{e}}}}$, respectively. ${ }^{1}$ We define the product space $Z_{\mathrm{e}} \equiv X_{\mathrm{e}} \times Y_{\mathrm{e}}$, with inner product $(\cdot, \cdot)_{Z_{\mathrm{e}}} \equiv(\cdot, \cdot)_{X_{\mathrm{e}}}+(\cdot, \cdot)_{Y_{\mathrm{e}}}$ and norm $\|\cdot\|_{Z_{\mathrm{e}}}=\sqrt{(\cdot, \cdot)_{Z_{\mathrm{e}}}}$. The associated dual spaces are denoted by $X_{\mathrm{e}}^{\prime}, Y_{\mathrm{e}}^{\prime}$, and $Z_{\mathrm{e}}^{\prime}$.

Furthermore, let $\mathcal{D} \subset \mathbb{R}^{n}$ be a prescribed $n$-dimensional, compact parameter set. For any parameter $\mu \in \mathcal{D}$, we then consider the continuous bilinear forms $a(\cdot, \cdot ; \mu): X_{\mathrm{e}} \times X_{\mathrm{e}} \rightarrow \mathbb{R}$ and $b(\cdot, \cdot ; \mu): X_{\mathrm{e}} \times Y_{\mathrm{e}} \rightarrow \mathbb{R}^{2}$

$$
\begin{aligned}
& \gamma_{a}^{\mathrm{e}}(\mu) \equiv \sup _{u \in X_{\mathrm{e}}} \sup _{v \in X_{\mathrm{e}}} \frac{a(u, v ; \mu)}{\|u\|_{X_{\mathrm{e}}}\|v\|_{X_{\mathrm{e}}}}<\infty, \quad \forall \mu \in \mathcal{D}, \\
& \gamma_{b}^{\mathrm{e}}(\mu) \equiv \sup _{q \in Y_{\mathrm{e}}} \sup _{v \in X_{\mathrm{e}}} \frac{b(v, q ; \mu)}{\|q\|_{Y_{\mathrm{e}}}\|v\|_{X_{\mathrm{e}}}}<\infty, \quad \forall \mu \in \mathcal{D} .
\end{aligned}
$$

We moreover assume that $a(\cdot, \cdot ; \mu)$ is coercive on $X_{\mathrm{e}}$,

$$
\alpha_{a}^{\mathrm{e}}(\mu) \equiv \inf _{v \in X_{\mathrm{e}}} \frac{a(v, v ; \mu)}{\|v\|_{X_{\mathrm{e}}}^{2}}>0, \quad \forall \mu \in \mathcal{D}
$$

and that $b(\cdot, \cdot ; \mu)$ satisfies the inf-sup condition

$$
\beta_{\mathrm{Br}}^{\mathrm{e}}(\mu) \equiv \inf _{q \in Y_{\mathrm{e}}} \sup _{v \in X_{\mathrm{e}}} \frac{b(v, q ; \mu)}{\|q\|_{Y_{\mathrm{e}}}\|v\|_{X_{\mathrm{e}}}}>0, \quad \forall \mu \in \mathcal{D} .
$$

By (2.1) and (2.3), the bilinear form $a(\cdot, \cdot ; \mu)$ provides with $\|\cdot\|_{X_{\mathrm{e}}, \mu} \equiv \sqrt{a(\cdot, \cdot ; \mu)}$ an energy norm on $X_{\mathrm{e}}$ that is equivalent to $\|\cdot\|_{X_{\mathrm{e}}}$ for any $\mu \in \mathcal{D}$. We shall refer to $\beta_{\mathrm{Br}}^{\mathrm{e}}(\mu)$ as the "exact Brezzi" inf-sup constant. We additionally define the "exact Babuška" inf-sup constant

$$
\beta_{\mathrm{Ba}}^{\mathrm{e}}(\mu) \equiv \inf _{(u, p) \in Z_{\mathrm{e}}} \sup _{(v, q) \in Z_{\mathrm{e}}} \frac{a(u, v ; \mu)+b(v, p ; \mu)+b(u, q ; \mu)}{\|(u, p)\|_{Z_{\mathrm{e}}}\|(v, q)\|_{Z_{\mathrm{e}}}}, \quad \forall \mu \in \mathcal{D} ;
$$

it follows that $\beta_{\mathrm{Ba}}^{\mathrm{e}}(\mu)>0$ for all $\mu \in \mathcal{D}$ from the assumptions $(2.1)-(2.4)$ (see $\left.[5,12,27]\right)$.

We now consider the following variational problem: For any given $\mu \in \mathcal{D}$, we find $\left(u_{\mathrm{e}}(\mu), p_{\mathrm{e}}(\mu)\right) \in X_{\mathrm{e}} \times Y_{\mathrm{e}}$ such that

$$
\begin{aligned}
a\left(u_{\mathrm{e}}(\mu), v ; \mu\right)+b\left(v, p_{\mathrm{e}}(\mu) ; \mu\right) & =f(v ; \mu), & \forall v \in X_{\mathrm{e}}, \\
b\left(u_{\mathrm{e}}(\mu), q ; \mu\right) & =g(q ; \mu), \quad & \forall q \in Y_{\mathrm{e}},
\end{aligned}
$$

where $f(\cdot ; \mu)$ and $g(\cdot ; \mu)$ are bounded linear functionals in $X_{\mathrm{e}}^{\prime}$ and $Y_{\mathrm{e}}^{\prime}$, respectively. From the results of Brezzi [12] (see, e.g., also [13, 27, 36]), it is well-known that under the assumptions $(2.1),(2.2),(2.3)$, and (2.4), the above problem (2.6) is well-posed and has a unique solution for any $f(\cdot ; \mu) \in X_{\mathrm{e}}^{\prime}, g(\cdot ; \mu) \in Y_{\mathrm{e}}^{\prime}$. We recall that if the bilinear form $a(\cdot, \cdot ; \mu)$ is in addition

\footnotetext{
${ }^{1}$ Here and in the following, the subscript e denotes "exact".

${ }^{2}$ For clarity of exposition, we suppress the obvious requirement of nonzero elements in the denominators.
} 
symmetric for any $\mu \in \mathcal{D}$, the solution $\left(u_{\mathrm{e}}(\mu), p_{\mathrm{e}}(\mu)\right)$ to (2.6) corresponds to a saddle point of the Lagrangian functional

$$
\mathcal{L}(v, q ; \mu) \equiv \frac{1}{2} a(v, v ; \mu)+b(v, q ; \mu)-f(v ; \mu)-g(q ; \mu), \quad \forall(v, q) \in Z_{\mathrm{e}} .
$$

Therefore, even though we in fact consider a more general (not necessarily symmetric) case, we call (2.6) a parametrized saddle point problem.

We pause at this point to briefly comment on the nature of the parameter dependence of our linear and bilinear forms. The efficiency of the reduced basis method relies on an OfflineOnline computational decomposition strategy (see §2.6) that requires that all bilinear and linear forms in (2.6) depend affinely on the parameter $\mu$. For instance, we assume that for some $Q_{a} \in \mathbb{N}$, the bilinear form $a(\cdot, \cdot ; \mu)$ can be written as

$$
a(u, v ; \mu)=\sum_{k=1}^{Q_{a}} \Theta_{a}^{k}(\mu) a^{k}(u, v), \quad \forall u, v \in X_{\mathrm{e}},
$$

where for $1 \leq k \leq Q_{a}$, the parameter-dependent coefficient functions $\Theta_{a}^{k}(\mu)$ are continuous over the parameter set $\mathcal{D}$ and the parameter-independent bilinear forms $a^{k}(\cdot, \cdot)$ are continuous on $X_{\mathrm{e}} \times X_{\mathrm{e}}$. We assume analogous representations for the bilinear form $b(\cdot, \cdot ; \mu)$ and the linear functionals $f(\cdot ; \mu)$ and $g(\cdot ; \mu)$.

\subsubsection{Truth Approximation}

We now introduce a high-fidelity "truth" approximation upon which our reduced basis approximation will subsequently be built. To this end, let $X$ and $Y$ denote finite-dimensional subspaces of $X_{\mathrm{e}}$ and $Y_{\mathrm{e}}$, respectively. We define the product space $Z \equiv X \times Y$ and denote by $\mathcal{N}$ the dimension of $Z$. We emphasize that the dimension $\mathcal{N}$ is typically very large. These "truth" approximation subspaces inherit the inner products and norms of the exact spaces: $(\cdot, \cdot)_{X} \equiv(\cdot, \cdot)_{X_{\mathrm{e}}},\|\cdot\|_{X} \equiv\|\cdot\|_{X_{\mathrm{e}}},(\cdot, \cdot)_{Y} \equiv(\cdot, \cdot)_{Y_{\mathrm{e}}},\|\cdot\|_{Y} \equiv\|\cdot\|_{Y_{\mathrm{e}}}$, and $(\cdot, \cdot)_{Z} \equiv(\cdot, \cdot)_{Z_{\mathrm{e}}}$, $\|\cdot\|_{Z} \equiv\|\cdot\|_{Z_{\mathrm{e}}} \cdot$

The continuity and coercivity properties (2.1), (2.2), and (2.3) are clearly passed on to the "truth" approximation spaces,

$$
\begin{aligned}
\gamma_{a}(\mu) & \equiv \sup _{u \in X} \sup _{v \in X} \frac{a(u, v ; \mu)}{\|u\|_{X}\|v\|_{X}}<\infty, \quad \forall \mu \in \mathcal{D}, \\
\gamma_{b}(\mu) & \equiv \sup _{q \in Y} \sup _{v \in X} \frac{b(v, q ; \mu)}{\|q\|_{Y}\|v\|_{X}}<\infty, \quad \forall \mu \in \mathcal{D}, \\
\alpha_{a}(\mu) & \equiv \inf _{v \in X} \frac{a(v, v ; \mu)}{\|v\|_{X}^{2}}>0, \quad \forall \mu \in \mathcal{D} ;
\end{aligned}
$$

thus, $\|\cdot\|_{X, \mu} \equiv\|\cdot\|_{X_{\mathrm{e}}, \mu}$ defines a norm on $X$ that is equivalent to $\|\cdot\|_{X}$ for any $\mu \in \mathcal{D}$. We now further assume that the approximation spaces $X$ and $Y$ are chosen such that they satisfy the Ladyzhenskaya-Babuška-Brezzi (LBB) inf-sup condition (see, e.g., [13])

$$
\beta_{\mathrm{Br}}(\mu) \equiv \inf _{q \in Y} \sup _{v \in X} \frac{b(v, q ; \mu)}{\|q\|_{Y}\|v\|_{X}} \geq \beta_{\mathrm{Br}}^{0}(\mu)>0, \quad \forall \mu \in \mathcal{D}
$$


where $\beta_{\mathrm{Br}}^{0}(\mu)$ is a constant independent of the dimension $\mathcal{N}$. We refer to $\beta_{\mathrm{Br}}(\mu)$ as the "truth Brezzi" inf-sup constant. We shall also consider the "truth Babuška" inf-sup constant

$$
\beta_{\mathrm{Ba}}(\mu) \equiv \inf _{(u, p) \in Z} \sup _{(v, q) \in Z} \frac{a(u, v ; \mu)+b(v, p ; \mu)+b(u, q ; \mu)}{\|(u, p)\|_{Z}\|(v, q)\|_{Z}}, \quad \forall \mu \in \mathcal{D} ;
$$

again, it follows from $(2.8)-(2.11)$ that $\beta_{\mathrm{Ba}}(\mu)>0$ for all $\mu \in \mathcal{D}$. In particular, one can show that $\beta_{\mathrm{Br}}(\mu) \geq \beta_{\mathrm{Ba}}(\mu) \geq C\left(\alpha_{a}(\mu), \gamma_{a}(\mu), \beta_{\mathrm{Br}}(\mu)\right)>0$ for all $\mu \in \mathcal{D}$; see, e.g., [109] for an explicit representation of $C\left(\alpha_{a}(\mu), \gamma_{a}(\mu), \beta_{\mathrm{Br}}(\mu)\right)$.

We now define our "truth" approximations to be the Galerkin projections of $u_{\mathrm{e}}(\mu) \in X_{\mathrm{e}}$ and $p_{\mathrm{e}}(\mu) \in Y_{\mathrm{e}}$ onto $X$ and $Y$, respectively: Given any $\mu \in \mathcal{D}$, we find $(u(\mu), p(\mu)) \in X \times Y$ such that

$$
\begin{aligned}
a(u(\mu), v ; \mu)+b(v, p(\mu) ; \mu) & =f(v ; \mu), & & \forall v \in X, \\
b(u(\mu), q ; \mu) & =g(q ; \mu), & & \forall q \in Y .
\end{aligned}
$$

As for the exact problem in $\S 2.2 .1$, it follows from (2.8), (2.9), (2.10), and (2.11) that the "truth" problem (2.13) has a unique solution for any $f(\cdot ; \mu) \in X_{\mathrm{e}}^{\prime}, g(\cdot ; \mu) \in Y_{\mathrm{e}}^{\prime}$. The bilinear forms $a(\cdot, \cdot ; \mu)$ and $b(\cdot, \cdot ; \mu)$ define bounded linear operators $A(\mu): X \rightarrow X^{\prime}, B(\mu): X \rightarrow Y^{\prime}$ and its transpose $B(\mu)^{t}: Y \rightarrow X^{\prime}$ by

$$
\begin{array}{llrl}
\langle A(\mu) u, v\rangle & =a(u, v ; \mu), & & \forall u, v \in X, \\
\langle B(\mu) v, q\rangle & =b(v, q ; \mu)=\left\langle B(\mu)^{t} q, v\right\rangle, & & \forall v \in X, \forall q \in Y ;
\end{array}
$$

here, $\langle\cdot, \cdot\rangle$ denotes the respective dual pairing. The "truth" system (2.13) can thus be equivalently written as

$$
\begin{aligned}
& A(\mu) u(\mu)+B(\mu)^{t} p(\mu)=f(\mu) \text { in } X^{\prime} \text {, } \\
& B(\mu) u(\mu)=g(\mu) \text { in } Y^{\prime},
\end{aligned}
$$

where $\left.f(\mu) \equiv f(\cdot ; \mu)\right|_{X} \in X^{\prime}$ and $\left.g(\mu) \equiv g(\cdot ; \mu)\right|_{Y} \in Y^{\prime}$ for all $\mu \in \mathcal{D}$.

\subsection{Reduced Basis Approximation}

We now turn to the reduced basis method, discussing the approximation procedure, rigorous a posteriori error estimators, and the construction of stable approximation spaces.

\subsubsection{Formulation}

Let us suppose for now that we are given a set of nested, low-dimensional reduced basis approximation subspaces $X_{N} \subset X_{N+1} \subset X$ and $Y_{N} \subset Y_{N+1} \subset Y, N \in \mathbb{N}_{\max } \equiv\left\{1, \ldots, N_{\max }\right\}$. We denote by $N_{X}$ and $N_{Y}$ the dimensions of $X_{N}$ and $Y_{N}$, respectively, and the total dimension of $Z_{N} \equiv X_{N} \times Y_{N}$ by $N_{Z} \equiv N_{X}+N_{Y}$. The subspaces $X_{N}, Y_{N}$, and $Z_{N}$ again inherit all inner products and norms of $X, Y$, and $Z$, respectively. The reduced basis approximation is then defined as the Galerkin projection onto these low-dimensional subspaces: For any given $\mu \in \mathcal{D}$, we find $u_{N}(\mu) \in X_{N}$ and $p_{N}(\mu) \in Y_{N}$ such that

$$
\begin{array}{rlrl}
a\left(u_{N}(\mu), v_{N} ; \mu\right)+b\left(v_{N}, p_{N}(\mu) ; \mu\right) & =f\left(v_{N} ; \mu\right), & & \forall v_{N} \in X_{N}, \\
b\left(u_{N}(\mu), q_{N} ; \mu\right) & =g\left(q_{N} ; \mu\right), \quad & \forall q_{N} \in Y_{N} .
\end{array}
$$


Written in operator notation, the discrete reduced basis system reads

$$
\begin{aligned}
& A_{N}(\mu) u_{N}(\mu)+B_{N}(\mu)^{t} p_{N}(\mu)=f_{N}(\mu) \quad \text { in } X_{N}^{\prime}, \\
& B_{N}(\mu) u_{N}(\mu)=g_{N}(\mu) \text { in } Y_{N}^{\prime} \text {, }
\end{aligned}
$$

where $\left.f_{N}(\mu) \equiv f(\cdot ; \mu)\right|_{X_{N}} \in X_{N}^{\prime},\left.g_{N}(\mu) \equiv g(\cdot ; \mu)\right|_{Y_{N}} \in Y_{N}^{\prime}$, and the bounded linear operators $A_{N}(\mu): X_{N} \rightarrow X_{N}^{\prime}, B_{N}(\mu): X_{N} \rightarrow Y_{N}^{\prime}$ and its transpose $B_{N}(\mu)^{t}: Y_{N} \rightarrow X_{N}^{\prime}$ are given by

$$
\begin{array}{lll}
\left\langle A_{N}(\mu) u_{N}, v_{N}\right\rangle=a\left(u_{N}, v_{N} ; \mu\right), & \forall u_{N}, v_{N} \in X_{N}, \\
\left\langle B_{N}(\mu) v_{N}, q_{N}\right\rangle=b\left(v_{N}, q_{N} ; \mu\right)=\left\langle B_{N}(\mu)^{t} q_{N}, v_{N}\right\rangle, & \forall v_{N} \in X_{N}, \forall q_{N} \in Y_{N} .
\end{array}
$$

\subsubsection{Properties of the Discrete System}

We first briefly recall some fundamental properties of the discrete system (2.14). Note that we did not yet impose any special requirements on the reduced basis approximation spaces $X_{N}, Y_{N}$. For a given parameter $\mu \in \mathcal{D}$, the system (2.15), (2.16) is solvable if and only if $g_{N}(\mu)$ belongs to the range of the operator $B_{N}(\mu)$. In this case, we can construct $u_{N}(\mu) \in X_{N}$ satisfying (2.16) such that $f_{N}(\mu)-A_{N}(\mu) u_{N}(\mu)$ belongs to $\operatorname{im}\left(B_{N}(\mu)^{t}\right)$ and there exists $p_{N}(\mu) \in Y_{N}$ satisfying (2.15). The solution $u_{N}(\mu)$ is unique, $p_{N}(\mu)$ is in general determined only up to an element of $\operatorname{ker}\left(B_{N}(\mu)^{t}\right)$. The system (2.15), (2.16) is therefore uniquely solvable if and only if $g_{N}(\mu)$ belongs to the range of the operator $B_{N}(\mu)$ and the operator $B_{N}(\mu)^{t}$ is injective. In the specific case of finite-dimensional spaces $X_{N}$ and $Y_{N}, B_{N}(\mu)^{t}$ is injective if and only if $B_{N}(\mu)$ is surjective. Since this clearly implies that $g_{N} \in \operatorname{im}\left(B_{N}(\mu)\right)$ for any $g_{N} \in Y_{N}^{\prime}$, we obtain the following: For a given parameter $\mu \in \mathcal{D}$, the system $(2.15),(2.16)$ is well-posed if and only if $B_{N}(\mu)$ is surjective. This may be equivalently expressed by the inf-sup condition

$$
\beta_{N}(\mu) \equiv \inf _{q_{N} \in Y_{N}} \sup _{v_{N} \in X_{N}} \frac{b\left(v_{N}, q_{N} ; \mu\right)}{\left\|q_{N}\right\|_{Y}\left\|v_{N}\right\|_{X}}=\inf _{q_{N} \in Y_{N}} \frac{\left\|B_{N}(\mu)^{t} q_{N}\right\|_{X_{N}^{\prime}}}{\left\|q_{N}\right\|_{Y}}>0 .
$$

(For further details on any of the steps above, we refer the reader to [12, 13] and Appendix B.) In the remainder of this work, a pair of approximation spaces $\left(X_{N}, Y_{N}\right)$ satisfying $(2.17)$ for any parameter value $\mu \in \mathcal{D}$ is called stable.

\subsubsection{A Priori Error Estimation}

We here consider a priori estimates for the errors in the reduced basis approximations. These shall also contribute to the construction of reduced basis approximation spaces in $\S 2.5$.

In this section, we assume that the low-dimensional reduced basis spaces $X_{N}, Y_{N}$ are constructed such that for any given parameter $\mu \in \mathcal{D}$, a solution $\left(u_{N}(\mu), p_{N}(\mu)\right) \in X_{N} \times Y_{N}$ to (2.14) exists. We then denote the errors in the reduced basis approximations $u_{N}(\mu) \in X_{N}$, $p_{N}(\mu) \in Y_{N}$, and $\left(u_{N}(\mu), p_{N}(\mu)\right) \in Z_{N}$ with respect to the truth approximations by

$$
\begin{aligned}
& e_{N}^{u}(\mu) \equiv u(\mu)-u_{N}(\mu) \in X \\
& e_{N}^{p}(\mu) \equiv p(\mu)-p_{N}(\mu) \in Y, \\
& e_{N}(\mu) \equiv\left(e_{N}^{u}(\mu), e_{N}^{p}(\mu)\right) \in Z .
\end{aligned}
$$


Proposition 2.3.1. For any given $\mu \in \mathcal{D}$ and $N \in \mathbb{N}_{\max }$, we have

$$
\left\|e_{N}^{u}(\mu)\right\|_{X} \leq\left(1+\frac{\gamma_{a}(\mu)}{\alpha_{a}(\mu)}\right) \inf _{\substack{v_{N} \in X_{N} \\ B_{N}(\mu) v_{N}=g_{N}(\mu)}}\left\|u(\mu)-v_{N}\right\|_{X}+\frac{\gamma_{b}(\mu)}{\alpha_{a}(\mu)} \inf _{q_{N} \in Y_{N}}\left\|p(\mu)-q_{N}\right\|_{Y}
$$

for the error in the reduced basis approximation $u_{N}(\mu)$; moreover, if the spaces $X_{N}, Y_{N}$ are stable (see §2.3.2), we also obtain

$$
\left\|e_{N}^{p}(\mu)\right\|_{Y} \leq\left(1+\frac{\gamma_{b}(\mu)}{\beta_{N}(\mu)}\right) \inf _{q_{N} \in Y_{N}}\left\|p(\mu)-q_{N}\right\|_{Y}+\frac{\gamma_{a}(\mu)}{\beta_{N}(\mu)}\left\|e_{N}^{u}(\mu)\right\|_{X}
$$

for the error in the reduced basis approximation $p_{N}(\mu)$, and

$$
\inf _{\substack{v_{N} \in X_{N} \\ B_{N}(\mu) v_{N}=g_{N}(\mu)}}\left\|u(\mu)-v_{N}\right\|_{X} \leq\left(1+\frac{\gamma_{b}(\mu)}{\beta_{N}(\mu)}\right) \inf _{v_{N} \in X_{N}}\left\|u(\mu)-v_{N}\right\|_{X} .
$$

Proof. The statement directly follows from arguments presented in [13] applied to the reduced basis context.

In the special case of a symmetric problem, we may also derive the following result.

Proposition 2.3.2. Let $a(\cdot, \cdot ; \mu)$ be symmetric for all $\mu \in \mathcal{D}$. Then, for any given $\mu \in \mathcal{D}$ and $N \in \mathbb{N}_{\max }$, we have

$$
\left\|e_{N}^{u}(\mu)\right\|_{X} \leq 2 \sqrt{\frac{\gamma_{a}(\mu)}{\alpha_{a}(\mu)}} \inf _{\substack{v_{N} \in X_{N} \\ B_{N}(\mu) v_{N}=g_{N}(\mu)}}\left\|u(\mu)-v_{N}\right\|_{X}+\frac{\gamma_{b}(\mu)}{\alpha_{a}(\mu)} \inf _{q_{N} \in Y_{N}}\left\|p(\mu)-q_{N}\right\|_{Y}
$$

moreover, if the spaces $X_{N}, Y_{N}$ are stable (see §2.3.2), we also obtain

$$
\begin{aligned}
\left\|e_{N}^{p}(\mu)\right\|_{Y} \leq\left[1+\frac{\gamma_{b}(\mu)}{\beta_{N}(\mu)}\left(1+\sqrt{\frac{\gamma_{a}(\mu)}{\alpha_{a}(\mu)}}\right)\right] & \inf _{q_{N} \in Y_{N}}\left\|p(\mu)-q_{N}\right\|_{Y} \\
& +2 \frac{\gamma_{a}(\mu)}{\beta_{N}(\mu)} \inf _{\substack{v_{N} \in X_{N} \\
B_{N}(\mu) v_{N}=g_{N}(\mu)}}\left\|u(\mu)-v_{N}\right\|_{X} .
\end{aligned}
$$

Proof. We here use techniques very similar to those presented in [13]. Take any parameter $\mu \in \mathcal{D}$ and $N \in \mathbb{N}_{\max }$. We note that, as a continuous, symmetric, and coercive bilinear form, $a(\cdot, \cdot ; \mu)$ defines an inner product on $X$, and recall that the associated norm is denoted by $\|\cdot\|_{X, \mu}=\sqrt{a(\cdot, \cdot ; \mu)}$. By the definition of the reduced basis approximation in $\S 2.3 .1$ as the Galerkin projection of $(u(\mu), p(\mu))$ onto $X_{N} \times Y_{N}$, the errors $e_{N}^{u}(\mu)$ and $e_{N}^{p}(\mu)$ satisfy

$$
a\left(e_{N}^{u}(\mu), v_{N} ; \mu\right)+b\left(v_{N}, e_{N}^{p}(\mu) ; \mu\right)=0, \quad \forall v_{N} \in X_{N}
$$


First, we prove that $(2.22)$ holds true. For any $v_{N} \in X_{N}$ such that $B_{N}(\mu) v_{N}=g_{N}(\mu)$ in $Y_{N}^{\prime}$, we have $v_{N}-u_{N}(\mu) \in \operatorname{ker}\left(B_{N}(\mu)\right)$ and

$$
\begin{aligned}
\left\|v_{N}-u_{N}(\mu)\right\|_{X, \mu} & =\frac{a\left(v_{N}-u_{N}(\mu), v_{N}-u_{N}(\mu) ; \mu\right)}{\left\|v_{N}-u_{N}(\mu)\right\|_{X, \mu}} \\
& \leq \sup _{w_{N} \in \operatorname{ker}\left(B_{N}(\mu)\right)} \frac{a\left(v_{N}-u_{N}(\mu), w_{N} ; \mu\right)}{\left\|w_{N}\right\|_{X, \mu}} \\
& =\sup _{w_{N} \in \operatorname{ker}\left(B_{N}(\mu)\right)} \frac{a\left(v_{N}-u(\mu), w_{N} ; \mu\right)+a\left(e_{N}^{u}(\mu), w_{N} ; \mu\right)}{\left\|w_{N}\right\|_{X, \mu}} \\
& =\sup _{w_{N} \in \operatorname{ker}\left(B_{N}(\mu)\right)} \frac{a\left(v_{N}-u(\mu), w_{N} ; \mu\right)-b\left(w_{N}, e_{N}^{p}(\mu) ; \mu\right)}{\left\|w_{N}\right\|_{X, \mu}},
\end{aligned}
$$

where the last equality follows from (2.24). For $w_{N} \in \operatorname{ker}\left(B_{N}(\mu)\right), b\left(w_{N}, p_{N}(\mu) ; \mu\right)=$ $b\left(w_{N}, q_{N} ; \mu\right)=0$ holds for all $q_{N} \in Y_{N}$. Inserting this in the inequality above yields, for any $q_{N} \in Y_{N}$,

$$
\begin{aligned}
\left\|v_{N}-u_{N}(\mu)\right\|_{X, \mu} & \leq \sup _{w_{N} \in \operatorname{ker}\left(B_{N}(\mu)\right)} \frac{a\left(v_{N}-u(\mu), w_{N} ; \mu\right)-b\left(w_{N}, p(\mu)-q_{N} ; \mu\right)}{\left\|w_{N}\right\|_{X, \mu}} \\
& \leq\left\|v_{N}-u(\mu)\right\|_{X, \mu}+\frac{\gamma_{b}(\mu)}{\sqrt{\alpha_{a}(\mu)}}\left\|p(\mu)-q_{N}\right\|_{Y},
\end{aligned}
$$

where the latter is obtained from the Cauchy-Schwarz inequality for the inner product $a(\cdot, \cdot ; \mu),(2.9)$, and $(2.10)$. Using the triangle inequality and $(2.25)$,

$$
\begin{aligned}
\left\|e_{N}^{u}(\mu)\right\|_{X, \mu} & \leq\left\|u(\mu)-v_{N}\right\|_{X, \mu}+\left\|v_{N}-u_{N}(\mu)\right\|_{X, \mu} \\
& \leq 2\left\|u(\mu)-v_{N}\right\|_{X, \mu}+\frac{\gamma_{b}(\mu)}{\sqrt{\alpha_{a}(\mu)}}\left\|p(\mu)-q_{N}\right\|_{Y}
\end{aligned}
$$

the a priori stability estimate (2.22) then follows from (2.26), (2.8), and (2.10).

We now turn to (2.23). Assuming that $X_{N}$ and $Y_{N}$ are stable, the inf-sup condition (2.17) provides

$$
\beta_{N}(\mu)\left\|q_{N}-p_{N}(\mu)\right\|_{Y} \leq \sup _{v_{N} \in X_{N}} \frac{b\left(v_{N}, q_{N}-p_{N}(\mu) ; \mu\right)}{\left\|v_{N}\right\|_{X}}, \quad \forall q_{N} \in Y_{N} .
$$

For any $v_{N} \in X_{N}$ and $q_{N} \in Y_{N}$, we moreover have

$$
\begin{aligned}
b\left(v_{N}, q_{N}-p_{N}(\mu) ; \mu\right) & =b\left(v_{N}, q_{N}-p(\mu) ; \mu\right)+b\left(v_{N}, e_{N}^{p}(\mu) ; \mu\right) \\
& =b\left(v_{N}, q_{N}-p(\mu) ; \mu\right)-a\left(e_{N}^{u}(\mu), v_{N} ; \mu\right),
\end{aligned}
$$

where the last equality follows from (2.24). Applying this to (2.27), together with the Cauchy-Schwarz inequality for $a(\cdot, \cdot ; \mu)$ and $(2.8)$, we obtain

$$
\left\|q_{N}-p_{N}(\mu)\right\|_{Y} \leq \frac{\gamma_{b}(\mu)}{\beta_{N}(\mu)}\left\|p(\mu)-q_{N}\right\|_{Y}+\frac{\sqrt{\gamma_{a}(\mu)}}{\beta_{N}(\mu)}\left\|e_{N}^{u}(\mu)\right\|_{X, \mu}, \quad \forall q_{N} \in Y_{N} ;
$$

the a priori error estimate (2.23) thus holds again from the triangle inequality, (2.28), (2.26), and (2.8). 


\subsection{A Posteriori Error Estimation}

We now aim to develop not only efficient reduced order approximations, but also a posteriori error estimates that are rigorous, sharp, and computationally inexpensive.

In this section, we again assume that the low-dimensional reduced basis approximation spaces $X_{N}, Y_{N}$ are constructed such that for any given parameter $\mu \in \mathcal{D}$, a solution $\left(u_{N}(\mu), p_{N}(\mu)\right) \in X_{N} \times Y_{N}$ to (2.14) exists. We may then derive upper bounds as required for the errors $e_{N}^{u}(\mu)$ and $e_{N}^{p}(\mu)$ in the reduced basis approximations $u_{N}(\mu)$ and $p_{N}(\mu)$, respectively. Symmetric saddle point problems shall be discussed as a special case in which these bounds can be further sharpened.

\subsubsection{Ingredients}

To formulate rigorous and computationally inexpensive upper bounds for the respective errors defined in (2.18), we first have to introduce further ingredients. The first set of ingredients consists of computationally (Online-)efficient lower and upper bounds to the truth continuity and coercivity constants (2.8) and (2.10),

$$
\begin{aligned}
& \gamma_{a}^{\mathrm{LB}}(\mu) \leq \gamma_{a}(\mu) \leq \gamma_{a}^{\mathrm{UB}}(\mu), \quad \forall \mu \in \mathcal{D}, \\
& \alpha_{a}^{\mathrm{LB}}(\mu) \leq \alpha_{a}(\mu) \leq \alpha_{a}^{\mathrm{UB}}(\mu),
\end{aligned}
$$

and to the truth Brezzi and Babuška inf-sup constants (2.11) and (2.12),

$$
\begin{aligned}
& \beta_{\mathrm{Br}}^{\mathrm{LB}}(\mu) \leq \beta_{\mathrm{Br}}(\mu) \leq \beta_{\mathrm{Br}}^{\mathrm{UB}}(\mu), \quad \forall \mu \in \mathcal{D} . \\
& \beta_{\mathrm{Ba}}^{\mathrm{LB}}(\mu) \leq \beta_{\mathrm{Ba}}(\mu) \leq \beta_{\mathrm{Ba}}^{\mathrm{UB}}(\mu),
\end{aligned}
$$

The second set of ingredients consists of dual norms of the residuals associated with the reduced basis approximation,

$$
\left\|r_{N}^{1}(\cdot ; \mu)\right\|_{X^{\prime}}=\sup _{v \in X} \frac{r_{N}^{1}(v ; \mu)}{\|v\|_{X}}, \quad\left\|r_{N}^{2}(\cdot ; \mu)\right\|_{Y^{\prime}}=\sup _{q \in Y} \frac{r_{N}^{2}(q ; \mu)}{\|q\|_{Y}}
$$

where, for all $\mu \in \mathcal{D}, r_{N}^{1}(\cdot ; \mu) \in X^{\prime}$ and $r_{N}^{2}(\cdot ; \mu) \in Y^{\prime}$ are defined as

$$
\begin{array}{lll}
r_{N}^{1}(v ; \mu) \equiv f(v ; \mu)-a\left(u_{N}(\mu), v ; \mu\right)-b\left(v, p_{N}(\mu) ; \mu\right), & \forall v \in X, \\
r_{N}^{2}(q ; \mu) \equiv g(q ; \mu)-b\left(u_{N}(\mu), q ; \mu\right), & \forall q \in Y .
\end{array}
$$

The total residual is then given by $r_{N}((v, q) ; \mu) \equiv r_{N}^{1}(v ; \mu)+r_{N}^{2}(q ; \mu)$ for all $(v, q) \in Z$, with its dual norm (see Appendix B)

$$
\left\|r_{N}(\cdot ; \mu)\right\|_{Z^{\prime}}=\sup _{(v, q) \in Z} \frac{r_{N}((v, q) ; \mu)}{\|(v, q)\|_{Z}}=\sqrt{\left\|r_{N}^{1}(\cdot ; \mu)\right\|_{X^{\prime}}^{2}+\left\|r_{N}^{2}(\cdot ; \mu)\right\|_{Y^{\prime}}^{2}}, \quad \forall \mu \in \mathcal{D} .
$$

\subsubsection{Error Bound Formulation}

We may now formulate a posteriori error bounds for the respective errors (2.18) in the reduced basis approximation. The following proposition represents a well-known result for noncoercive problems $[72,76,79,106]$. 
Proposition 2.4.1. For any given $\mu \in \mathcal{D}, N \in \mathbb{N}_{\max }$, and $\beta_{\mathrm{Ba}}^{\mathrm{LB}}(\mu)$ satisfying (2.30), we define

$$
\Delta_{N}^{\mathrm{Ba}}(\mu) \equiv \frac{\left\|r_{N}(\cdot ; \mu)\right\|_{Z^{\prime}}}{\beta_{\mathrm{Ba}}^{\mathrm{LB}}(\mu)} .
$$

Then, $\Delta_{N}^{\mathrm{Ba}}(\mu)$ is an upper bound for the error $e_{N}(\mu)$ such that

$$
\left\|e_{N}(\mu)\right\|_{Z} \leq \Delta_{N}^{\mathrm{Ba}}(\mu), \quad \forall \mu \in \mathcal{D}, \forall N \in \mathbb{N}_{\max }
$$

Proof. The above error bound directly follows from the Banach-Nečas-Babuška theorem $[5,27]$ and $(2.30)$.

Since many engineering outputs of interest such as average vorticity or pressure drop depend on either the primal variable (velocity) $u(\mu)$ or the Lagrange multiplier (pressure) $p(\mu)$, we aim to develop separate bounds for the errors in the reduced basis approximations $u_{N}(\mu)$ and $p_{N}(\mu)$. Noting that a posteriori reduced basis error bounds for coercive and general noncoercive problems can be derived from standard stability results, we apply Brezzi's theory for saddle point problems [12] to our problem setting. Since some aspects of this theory are important also to the construction of reduced basis approximation spaces in $\S 2.5$ and $\S 2.7$, we briefly summarize the proof below.

Proposition 2.4.2. For any given $\mu \in \mathcal{D}, N \in \mathbb{N}_{\max }$, and $\alpha_{a}^{\mathrm{LB}}(\mu), \gamma_{a}^{\mathrm{UB}}(\mu), \beta_{\mathrm{Br}}^{\mathrm{LB}}(\mu)$ satisfying (2.29) and (2.30), we define

$$
\begin{aligned}
& \Delta_{N}^{u, \mathrm{Br}}(\mu) \equiv \frac{\left\|r_{N}^{1}(\cdot ; \mu)\right\|_{X^{\prime}}}{\alpha_{a}^{\mathrm{LB}}(\mu)}+\left(1+\frac{\gamma_{a}^{\mathrm{UB}}(\mu)}{\alpha_{a}^{\mathrm{LB}}(\mu)}\right) \frac{\left\|r_{N}^{2}(\cdot ; \mu)\right\|_{Y^{\prime}}}{\beta_{\mathrm{Br}}^{\mathrm{LB}}(\mu)}, \\
& \Delta_{N}^{p, \operatorname{Br}}(\mu) \equiv \frac{\left\|r_{N}^{1}(\cdot ; \mu)\right\|_{X^{\prime}}}{\beta_{\mathrm{Br}}^{\mathrm{LB}}(\mu)}+\frac{\gamma_{a}^{\mathrm{UB}}(\mu)}{\beta_{\mathrm{Br}}^{\mathrm{LB}}(\mu)} \Delta_{N}^{u, \mathrm{Br}}(\mu) .
\end{aligned}
$$

Then, $\Delta_{N}^{u, \operatorname{Br}}(\mu)$ and $\Delta_{N}^{p, \operatorname{Br}}(\mu)$ are upper bounds for the errors $e_{N}^{u}(\mu)$ and $e_{N}^{p}(\mu)$ such that

$$
\left\|e_{N}^{u}(\mu)\right\|_{X} \leq \Delta_{N}^{u, \operatorname{Br}}(\mu), \quad\left\|e_{N}^{p}(\mu)\right\|_{Y} \leq \Delta_{N}^{p, \operatorname{Br}}(\mu), \quad \forall \mu \in \mathcal{D}, \forall N \in \mathbb{N}_{\max } .
$$

Proof. Let $\mu \in \mathcal{D}$ and $N \in \mathbb{N}_{\max }$. By (2.32), (2.33), and (2.13), the errors $e_{N}^{u}(\mu) \in X$ and $e_{N}^{p}(\mu) \in Y$ satisfy the equations

$$
\begin{aligned}
a\left(e_{N}^{u}(\mu), v ; \mu\right)+b\left(v, e_{N}^{p}(\mu) ; \mu\right) & =r_{N}^{1}(v ; \mu), \quad \forall v \in X, \\
b\left(e_{N}^{u}(\mu), q ; \mu\right) & =r_{N}^{2}(q ; \mu), \quad \forall q \in Y .
\end{aligned}
$$

We first consider the error $e_{N}^{u}(\mu) \in X$ in the approximation of the primal variable. This may be uniquely decomposed into $e_{N}^{u}(\mu)=e_{N}^{0}(\mu)+e_{N}^{\perp}(\mu)$, where $e_{N}^{0}(\mu) \in \operatorname{ker}(B(\mu))$ and $e_{N}^{\perp}(\mu) \in \operatorname{ker}(B(\mu))^{\perp} \equiv\left\{v \in X \mid\left(v, v_{0}\right)_{X}=0\right.$ for all $\left.v_{0} \in \operatorname{ker}(B(\mu))\right\}$. From (2.40), $e_{N}^{0}(\mu) \in \operatorname{ker}(B(\mu))$ then solves

$$
a\left(e_{N}^{0}(\mu), v_{0} ; \mu\right)=r_{N}^{1}\left(v_{0} ; \mu\right)-a\left(e_{N}^{\perp}(\mu), v_{0} ; \mu\right), \quad \forall v_{0} \in \operatorname{ker}(B(\mu)),
$$


and it is thus bounded by

$$
\begin{aligned}
\left\|e_{N}^{0}(\mu)\right\|_{X} & \leq \frac{1}{\alpha_{a}(\mu)} \sup _{v_{0} \in \operatorname{ker}(B(\mu))} \frac{r_{N}^{1}\left(v_{0} ; \mu\right)-a\left(e_{N}^{\perp}(\mu), v_{0} ; \mu\right)}{\left\|v_{0}\right\|_{X}} \\
& \leq \frac{1}{\alpha_{a}(\mu)}\left(\left\|r_{N}^{1}(\cdot ; \mu)\right\|_{X^{\prime}}+\gamma_{a}(\mu)\left\|e_{N}^{\perp}(\mu)\right\|_{X}\right),
\end{aligned}
$$

using (2.8), (2.10), and the classical Lax-Milgram lemma (see, e.g., [27, 89]). By the LBB inf-sup condition (2.11), it is also true that (see, e.g., [12], [13, §II.1, Proposition 1.2])

$$
\beta_{\mathrm{Br}}(\mu)=\inf _{v \in \operatorname{ker}(B(\mu))^{\perp}} \sup _{q \in Y} \frac{b(v, q ; \mu)}{\|v\|_{X}\|q\|_{Y}} .
$$

Applied to $e_{N}^{\perp}(\mu) \in \operatorname{ker}(B(\mu))^{\perp}$, this yields

$$
\left\|e_{N}^{\perp}(\mu)\right\|_{X} \leq \frac{1}{\beta_{\mathrm{Br}}(\mu)} \sup _{q \in Y} \frac{b\left(e_{N}^{\perp}(\mu), q ; \mu\right)}{\|q\|_{Y}}=\frac{\left\|r_{N}^{2}(\cdot ; \mu)\right\|_{Y^{\prime}}}{\beta_{\mathrm{Br}}(\mu)}
$$

where the equality follows from (2.41) as $B(\mu) e_{N}^{\perp}(\mu)=B(\mu) e_{N}^{u}(\mu)$ in $Y^{\prime}$. We then obtain the bound (2.37) for $\left\|e_{N}^{u}(\mu)\right\|_{X}$ by combining (2.43), (2.45) and (2.29), (2.30).

We now turn to the error in the approximation of the Lagrange multiplier. From (2.11) and (2.40), we derive that

$$
\begin{aligned}
\left\|e_{N}^{p}(\mu)\right\|_{Y} & \leq \frac{1}{\beta_{\mathrm{Br}}(\mu)} \sup _{v \in X} \frac{b\left(v, e_{N}^{p}(\mu) ; \mu\right)}{\|v\|_{X}}=\frac{1}{\beta_{\mathrm{Br}}(\mu)} \sup _{v \in X} \frac{r_{N}^{1}(v ; \mu)-a\left(e_{N}^{u}(\mu), v ; \mu\right)}{\|v\|_{X}} \\
& \leq \frac{1}{\beta_{\mathrm{Br}}(\mu)}\left(\left\|r_{N}^{1}(\cdot ; \mu)\right\|_{X^{\prime}}+\gamma_{a}(\mu)\left\|e_{N}^{u}(\mu)\right\|_{X}\right) .
\end{aligned}
$$

Together with (2.37) and again (2.29), (2.30), this finally yields (2.38) and (2.39).

Clearly, we can now construct another rigorous upper bound $\Delta_{N}^{\mathrm{Br}}(\mu)$ for the combined error $e_{N}(\mu)=\left(e_{N}^{u}(\mu), e_{N}^{p}(\mu)\right)$ : From (2.39), we obtain

$$
\left\|e_{N}(\mu)\right\|_{Z} \leq \sqrt{\left(\Delta_{N}^{u, \mathrm{Br}}(\mu)\right)^{2}+\left(\Delta_{N}^{p, \operatorname{Br}}(\mu)\right)^{2}} \equiv \Delta_{N}^{\mathrm{Br}}(\mu), \quad \forall \mu \in \mathcal{D}, N \in \mathbb{N}_{\max }
$$

As a measure of the quality of the proposed error estimators, we introduce the associated effectivities

$$
\eta_{N}^{\mathrm{Ba}}(\mu) \equiv \frac{\Delta_{N}^{\mathrm{Ba}}(\mu)}{\left\|e_{N}(\mu)\right\|_{Z}}, \quad \eta_{N}^{\mathrm{Br}}(\mu) \equiv \frac{\Delta_{N}^{\mathrm{Br}}(\mu)}{\left\|e_{N}(\mu)\right\|_{Z}}, \quad \mu \in \mathcal{D}, N \in \mathbb{N}_{\max }
$$

Effectivities $\geq 1$ thus indicate that the error estimate is rigorous; effectivities close to unity indicate that the error estimate is sharp. The following corollary specifies this behavior for the error bounds derived above.

Corollary 2.4.3. For any given $\mu \in \mathcal{D}$ and $N \in \mathbb{N}_{\max }$, the effectivities of the a posteriori error bounds (2.35) and (2.47) satisfy

$$
1 \leq \eta_{N}^{\mathrm{Ba}}(\mu) \leq \frac{\gamma_{a}(\mu)+\gamma_{b}(\mu)}{\beta_{\mathrm{Ba}}^{\mathrm{LB}}(\mu)}, \quad 1 \leq \eta_{N}^{\mathrm{Br}}(\mu) \leq C_{1}(\mu)\left(\gamma_{a}(\mu)+\gamma_{b}(\mu)\right),
$$

where $C_{1}(\mu)>0$ is a constant depending on $\alpha_{a}^{\mathrm{LB}}(\mu), \gamma_{a}^{\mathrm{UB}}(\mu)$, and $\beta_{\mathrm{Br}}^{\mathrm{LB}}(\mu)$. 
Proof. Let $\mu$ be any parameter in $\mathcal{D}$ and $N \in \mathbb{N}_{\max }$. The error bounds are rigorous by (2.36) and (2.47), and it only remains to show the validity of the upper bounds for the effectivities.

First, we consider the effectivities associated with $\Delta_{N}^{\mathrm{Ba}}(\mu)$. By $(2.40),(2.41)$ and (2.8), (2.9), the total residual satisfies

$$
\begin{aligned}
r_{N}((v, q) ; \mu) & =r_{N}^{1}(v ; \mu)+r_{N}^{2}(q ; \mu)=a\left(e_{N}^{u}(\mu), v ; \mu\right)+b\left(v, e_{N}^{p}(\mu) ; \mu\right)+b\left(e_{N}^{u}(\mu), q ; \mu\right) \\
& \leq \gamma_{a}(\mu)\left\|e_{N}^{u}(\mu)\right\|_{X}\|v\|_{X}+\gamma_{b}(\mu)\|v\|_{X}\left\|e_{N}^{p}(\mu)\right\|_{Y}+\gamma_{b}(\mu)\left\|e_{N}^{u}(\mu)\right\|_{X}\|q\|_{Y} \\
& =\left(\begin{array}{c}
\|v\|_{X} \\
\|q\|_{Y}
\end{array}\right)\left(\begin{array}{cc}
\gamma_{a}(\mu) & \gamma_{b}(\mu) \\
\gamma_{b}(\mu) & 0
\end{array}\right)\left(\begin{array}{l}
\left\|e_{N}^{u}(\mu)\right\|_{X} \\
\left\|e_{N}^{p}(\mu)\right\|_{Y}
\end{array}\right), \quad \forall(v, q) \in Z .
\end{aligned}
$$

This may be estimated in terms of the Euclidean norm $\|\cdot\|_{2}$, i.e.,

$$
\begin{aligned}
r_{N}((v, q) ; \mu) & \leq\|(v, q)\|_{Z}\left\|\left(\begin{array}{cc}
\gamma_{a}(\mu) & \gamma_{b}(\mu) \\
\gamma_{b}(\mu) & 0
\end{array}\right)\right\|_{2}\left\|\left(e_{N}^{u}(\mu), e_{N}^{p}(\mu)\right)\right\|_{Z} \\
& \leq\left(\gamma_{a}(\mu)+\gamma_{b}(\mu)\right)\left\|e_{N}(\mu)\right\|_{Z}\|(v, q)\|_{Z}, \quad \forall(v, q) \in Z,
\end{aligned}
$$

where the last inequality follows from the relation $\|\cdot\|_{2} \leq \sqrt{\|\cdot\|_{1}\|\cdot\|_{\infty}}$ between matrix norms (see Appendix A). Thus, the first part of (2.49) holds from the definition of $\Delta_{N}^{\mathrm{Ba}}(\mu)$ in $(2.35)$.

We now turn to the effectivities associated with $\Delta_{N}^{\mathrm{Br}}(\mu)$. Again from $(2.40),(2.41)$, and $(2.8),(2.9)$, it is clearly also true that

$$
\begin{aligned}
\left\|r_{N}^{1}(\cdot ; \mu)\right\|_{X^{\prime}} & \leq \gamma_{a}(\mu)\left\|e_{N}^{u}(\mu)\right\|_{X}+\gamma_{b}(\mu)\left\|e_{N}^{p}(\mu)\right\|_{Y} \\
\left\|r_{N}^{2}(\cdot ; \mu)\right\|_{Y^{\prime}} & \leq \gamma_{b}(\mu)\left\|e_{N}^{u}(\mu)\right\|_{X} .
\end{aligned}
$$

Both residual dual norms (using either the Cauchy-Schwarz inequality or by adding terms) are then particularly bounded by

$$
\left(\gamma_{a}(\mu)^{2}+\gamma_{b}(\mu)^{2}\right)^{1 / 2}\left\|e_{N}(\mu)\right\|_{Z} \leq\left(\gamma_{a}(\mu)+\gamma_{b}(\mu)\right)\left\|e_{N}(\mu)\right\|_{Z}
$$

and we obtain for $\Delta_{N}^{u, \mathrm{Br}}(\mu)$ and $\Delta_{N}^{p, \mathrm{Br}}(\mu)$ that

$$
\begin{aligned}
& \Delta_{N}^{u, \operatorname{Br}}(\mu) \leq\left[\frac{1}{\alpha_{a}^{\mathrm{LB}}(\mu)}+\left(1+\frac{\gamma_{a}^{\mathrm{UB}}(\mu)}{\alpha_{a}^{\mathrm{LB}}(\mu)}\right) \frac{1}{\beta_{\mathrm{Br}}^{\mathrm{LB}}(\mu)}\right]\left(\gamma_{a}(\mu)+\gamma_{b}(\mu)\right)\left\|e_{N}(\mu)\right\|_{Z}, \\
& \Delta_{N}^{p, \mathrm{Br}}(\mu) \leq \frac{1}{\beta_{\mathrm{Br}}^{\mathrm{LB}}(\mu)}\left(1+\frac{\gamma_{a}^{\mathrm{UB}}(\mu)}{\alpha_{a}^{\mathrm{LB}}(\mu)}\right)\left(1+\frac{\gamma_{a}^{\mathrm{UB}}(\mu)}{\beta_{\mathrm{Br}}^{\mathrm{LB}}(\mu)}\right)\left(\gamma_{a}(\mu)+\gamma_{b}(\mu)\right)\left\|e_{N}(\mu)\right\|_{Z} ;
\end{aligned}
$$

the statement (2.49) now directly follows from the definition of $\Delta_{N}^{\mathrm{Br}}(\mu)$ in (2.47).

Remark 2.4.4. We here make some additional observations on the a posteriori error bounds given in Proposition 2.4.2 that shall be important also to the construction of reduced basis approximation spaces in $\S 2.5$.

It is clear from Corollary 2.4.3 that the residual dual norms (2.31) provide an a posteriori error estimator for $\left\|e_{N}(\mu)\right\|_{Z}$ that is not only reliable but also efficient (in the sense that 
the terms are used in the finite element community, i.e., bounding the error up to some constants from above and from below). Now, for any $\mu \in \mathcal{D}$ and $N \in \mathbb{N}_{\max }$, it follows from (2.42), (2.45), and (2.8) that the error $\left\|e_{N}^{u}(\mu)\right\|_{X}$ is in fact bounded by

$$
\left\|e_{N}^{u}(\mu)\right\|_{X} \leq \frac{1}{\alpha_{a}(\mu)} \sup _{v_{0} \in \operatorname{ker}(B(\mu))} \frac{r_{N}^{1}\left(v_{0} ; \mu\right)}{\left\|v_{0}\right\|_{X}}+\left(1+\frac{\gamma_{a}(\mu)}{\alpha_{a}(\mu)}\right) \frac{\left\|r_{N}^{2}(\cdot ; \mu)\right\|_{Y^{\prime}}}{\beta_{\mathrm{Br}}(\mu)}
$$

additionally using (2.51) and

$$
\sup _{v_{0} \in \operatorname{ker}(B(\mu))} \frac{r_{N}^{1}\left(v_{0} ; \mu\right)}{\left\|v_{0}\right\|_{X}}=\sup _{v_{0} \in \operatorname{ker}(B(\mu))} \frac{a\left(e_{N}^{u}(\mu), v_{0} ; \mu\right)}{\left\|v_{0}\right\|_{X}} \leq \gamma_{a}(\mu)\left\|e_{N}^{u}(\mu)\right\|_{X},
$$

we thus obtain a reliable and efficient a posteriori error estimator only for $\left\|e_{N}^{u}(\mu)\right\|_{X}$.

Remark 2.4.5. Following techniques used in [48] to obtain a priori stability estimates for the reduced basis approximations, we can derive an upper bound for the error in $u_{N}(\mu)$ that is similar to (2.37) but slightly differs in the weighting of the residual dual norms: For any $\mu \in \mathcal{D}$ and $N \in \mathbb{N}_{\max }$,

$$
\begin{aligned}
& \frac{1}{2 \alpha_{a}^{\mathrm{LB}}(\mu)}\left(\left\|r_{N}^{1}(\cdot ; \mu)\right\|_{X^{\prime}}+\frac{\gamma_{a}^{\mathrm{UB}}(\mu)}{\beta_{\mathrm{Br}}^{\mathrm{LB}}(\mu)}\left\|r_{N}^{2}(\cdot ; \mu)\right\|_{Y^{\prime}}\right) \\
& +\sqrt{\left[\frac{1}{2 \alpha_{a}^{\mathrm{LB}}(\mu)}\left(\left\|r_{N}^{1}(\cdot ; \mu)\right\|_{X^{\prime}}+\frac{\gamma_{a}^{\mathrm{UB}}(\mu)}{\beta_{\mathrm{Br}}^{\mathrm{LB}}(\mu)}\left\|r_{N}^{2}(\cdot ; \mu)\right\|_{Y^{\prime}}\right)\right]^{2}+\frac{\left\|r_{N}^{2}(\cdot ; \mu)\right\|_{Y^{\prime}}\left\|r_{N}^{1}(\cdot ; \mu)\right\|_{X^{\prime}}}{\alpha_{a}^{\mathrm{LB}}(\mu) \beta_{\mathrm{Br}}^{\mathrm{LB}}(\mu)}}
\end{aligned}
$$

is a rigorous upper bound for $\left\|e_{N}^{u}(\mu)\right\|_{X}$. The proof follows ideas in [48] and we only outline the main steps. From (2.10), (2.40), and (2.41), we have

$$
\begin{aligned}
\alpha_{a}(\mu)\left\|e_{N}^{u}(\mu)\right\|_{X}^{2} & \leq a\left(e_{N}^{u}(\mu), e_{N}^{u}(\mu) ; \mu\right)=r_{N}^{1}\left(e_{N}^{u}(\mu) ; \mu\right)-r_{N}^{2}\left(e_{N}^{p}(\mu) ; \mu\right) \\
& \leq\left\|r_{N}^{1}(\cdot ; \mu)\right\|_{X^{\prime}}\left\|e_{N}^{u}(\mu)\right\|_{X}+\left\|r_{N}^{2}(\cdot ; \mu)\right\|_{Y^{\prime}}\left\|e_{N}^{p}(\mu)\right\|_{Y} .
\end{aligned}
$$

Using further (2.46), we obtain the quadratic inequality

$$
\begin{aligned}
\alpha_{a}(\mu)\left\|e_{N}^{u}(\mu)\right\|_{X}^{2}-\left(\left\|r_{N}^{1}(\cdot ; \mu)\right\|_{X^{\prime}}+\frac{\gamma_{a}(\mu)}{\beta_{\mathrm{Br}}(\mu)}\left\|r_{N}^{2}(\cdot ; \mu)\right\|_{Y^{\prime}}\right)\left\|e_{N}^{u}(\mu)\right\|_{X} \\
-\frac{\left\|r_{N}^{2}(\cdot ; \mu)\right\|_{Y^{\prime}}\left\|r_{N}^{1}(\cdot ; \mu)\right\|_{X^{\prime}}}{\beta_{\operatorname{Br}}(\mu)} \leq 0,
\end{aligned}
$$

which we may solve for $\left\|e_{N}^{u}(\mu)\right\|_{X}$; this immediately leads to (2.54). Again combined with (2.46), this clearly also yields an associated error bound for $\left\|e_{N}^{p}(\mu)\right\|_{Y}$.

In the special case of a symmetric problem, the a posteriori error bounds given in Proposition 2.4 .2 can be improved. Now, also the following result holds true.

Proposition 2.4.6. Let $a(\cdot, \cdot ; \mu)$ be symmetric for all $\mu \in \mathcal{D}$. For any given $\mu \in \mathcal{D}$, $N \in \mathbb{N}_{\max }$, and $\alpha_{a}^{\mathrm{LB}}(\mu), \gamma_{a}^{\mathrm{UB}}(\mu), \beta_{\mathrm{Br}}^{\mathrm{LB}}(\mu)$ satisfying (2.29) and (2.30), we define

$$
\begin{aligned}
& \Delta_{N}^{u, \text {sym}}(\mu) \equiv \frac{\left\|r_{N}^{1}(\cdot ; \mu)\right\|_{X^{\prime}}}{\alpha_{a}^{\mathrm{LB}}(\mu)}+\sqrt{\frac{\gamma_{a}^{\mathrm{UB}}(\mu)}{\alpha_{a}^{\mathrm{LB}}(\mu)}} \frac{\left\|r_{N}^{2}(\cdot ; \mu)\right\|_{Y^{\prime}}}{\beta_{\mathrm{Br}}^{\mathrm{LB}}(\mu)}, \\
& \Delta_{N}^{p, \operatorname{sym}}(\mu) \equiv\left(1+\sqrt{\frac{\gamma_{a}^{\mathrm{UB}}(\mu)}{\alpha_{a}^{\mathrm{LB}}(\mu)}}\right) \frac{\left\|r_{N}^{1}(\cdot ; \mu)\right\|_{X^{\prime}}}{\beta_{\mathrm{Br}}^{\mathrm{LB}}(\mu)}+\frac{\gamma_{a}^{\mathrm{UB}}(\mu)}{\beta_{\mathrm{Br}}^{\mathrm{LB}}(\mu)} \frac{\left\|r_{N}^{2}(\cdot ; \mu)\right\|_{Y^{\prime}}}{\beta_{\mathrm{Br}}^{\mathrm{LB}}(\mu)} .
\end{aligned}
$$


Then, $\Delta_{N}^{u, \text { sym }}(\mu)$ and $\Delta_{N}^{p, \text { sym }}(\mu)$ are upper bounds for the errors $e_{N}^{u}(\mu)$ and $e_{N}^{p}(\mu)$ such that

$$
\left\|e_{N}^{u}(\mu)\right\|_{X} \leq \Delta_{N}^{u, \operatorname{sym}}(\mu)<\Delta_{N}^{u, \operatorname{Br}}(\mu), \quad\left\|e_{N}^{p}(\mu)\right\|_{Y} \leq \Delta_{N}^{p, \operatorname{sym}}(\mu)<\Delta_{N}^{p, \operatorname{Br}}(\mu),
$$

for all $\mu \in \mathcal{D}$ and $N \in \mathbb{N}_{\max }$.

Proof. Let $\mu$ be an arbitrary but fixed parameter in $\mathcal{D}$ and $N \in \mathbb{N}_{\max }$. We now proceed as in the proof of Proposition 2.4.2, only that we may now exploit the fact that $a(\cdot, \cdot ; \mu)$ defines an inner product on $X$. We recall that the associated norm is denoted by $\|\cdot\|_{X, \mu}=\sqrt{a(\cdot, \cdot ; \mu)}$.

The error $e_{N}^{u}(\mu) \in X$ in the approximation of the primal variable may now be uniquely decomposed into $e_{N}^{u}(\mu)=\tilde{e}_{N}^{0}(\mu)+\tilde{e}_{N}^{\perp}(\mu)$ where $\tilde{e}_{N}^{0}(\mu) \in \operatorname{ker}(B(\mu))$ and $\tilde{e}_{N}^{\perp}(\mu) \in X$ such that

$$
a\left(\tilde{e}_{N}^{\perp}(\mu), v_{0} ; \mu\right)=0, \quad \forall v_{0} \in \operatorname{ker}(B(\mu)) .
$$

From (2.40) and (2.58), $\tilde{e}_{N}^{0}(\mu) \in \operatorname{ker}(B(\mu))$ then solves

$$
a\left(\tilde{e}_{N}^{0}(\mu), v_{0} ; \mu\right)=r_{N}^{1}\left(v_{0} ; \mu\right)-a\left(\tilde{e}_{N}^{\perp}(\mu), v_{0} ; \mu\right)=r_{N}^{1}\left(v_{0} ; \mu\right), \quad \forall v_{0} \in \operatorname{ker}(B(\mu)) .
$$

Setting here $v_{0}=\tilde{e}_{N}^{0}(\mu)$, we have

$$
\begin{aligned}
\left\|\tilde{e}_{N}^{0}(\mu)\right\|_{X, \mu}^{2} & =r_{N}^{1}\left(\tilde{e}_{N}^{0}(\mu) ; \mu\right) \leq\left\|\tilde{e}_{N}^{0}(\mu)\right\|_{X} \sup _{v_{0} \in \operatorname{ker}(B(\mu))} \frac{r_{N}^{1}\left(v_{0} ; \mu\right)}{\left\|v_{0}\right\|_{X}} \\
& \leq \frac{1}{\sqrt{\alpha_{a}(\mu)}}\left\|\tilde{e}_{N}^{0}(\mu)\right\|_{X, \mu} \sup _{v_{0} \in \operatorname{ker}(B(\mu))} \frac{r_{N}^{1}\left(v_{0} ; \mu\right)}{\left\|v_{0}\right\|_{X}},
\end{aligned}
$$

where the last inequality follows from $(2.10)$. Hence, $\tilde{e}_{N}^{0}(\mu)$ is bounded by

$$
\left\|\tilde{e}_{N}^{0}(\mu)\right\|_{X, \mu} \leq \frac{1}{\sqrt{\alpha_{a}(\mu)}} \sup _{v_{0} \in \operatorname{ker}(B(\mu))} \frac{r_{N}^{1}\left(v_{0} ; \mu\right)}{\left\|v_{0}\right\|_{X}} \leq \frac{\left\|r_{N}^{1}(\cdot ; \mu)\right\|_{X^{\prime}}}{\sqrt{\alpha_{a}(\mu)}} .
$$

To obtain an upper bound for $\tilde{e}_{N}^{\perp}(\mu)$, we here consider the inf-sup constant

$$
\tilde{\beta}(\mu) \equiv \inf _{q \in Y} \sup _{v \in X} \frac{b(v, q ; \mu)}{\|q\|_{Y}\|v\|_{X, \mu}} .
$$

From (2.8) and (2.10), we have

$$
\frac{\beta_{\mathrm{Br}}(\mu)}{\sqrt{\gamma_{a}(\mu)}} \leq \tilde{\beta}(\mu) \leq \frac{\beta_{\mathrm{Br}}(\mu)}{\sqrt{\alpha_{a}(\mu)}},
$$

and thus particularly $\tilde{\beta}(\mu)>0$ by the LBB inf-sup condition (2.11). Consequently, analogous to $(2.44)$, also

$$
\tilde{\beta}(\mu)\|v\|_{X, \mu} \leq \sup _{q \in Y} \frac{b(v, q ; \mu)}{\|q\|_{Y}}
$$

holds true for any $v \in X$ such that $a\left(v, v_{0} ; \mu\right)=0$ for all $v_{0} \in \operatorname{ker}(B(\mu))$. Applied to $\tilde{e}_{N}^{\perp}(\mu)$ satisfying (2.58), this yields

$$
\left\|\tilde{e}_{N}^{\perp}(\mu)\right\|_{X, \mu} \leq \frac{1}{\tilde{\beta}(\mu)} \sup _{q \in Y} \frac{b\left(\tilde{e}_{N}^{\perp}(\mu), q ; \mu\right)}{\|q\|_{Y}}=\frac{\left\|r_{N}^{2}(\cdot ; \mu)\right\|_{Y^{\prime}}}{\tilde{\beta}(\mu)},
$$


where the equality again follows from $(2.41)$ as $B(\mu) \tilde{e}_{N}^{\perp}(\mu)=B(\mu) e_{N}^{u}(\mu)$ in $Y^{\prime}$. Now, using the triangle inequality, we may finally derive that

$$
\left\|e_{N}^{u}(\mu)\right\|_{X, \mu} \leq\left\|\tilde{e}_{N}^{0}(\mu)\right\|_{X, \mu}+\left\|\tilde{e}_{N}^{\perp}(\mu)\right\|_{X, \mu} \leq \frac{\left\|r_{N}^{1}(\cdot ; \mu)\right\|_{X^{\prime}}}{\sqrt{\alpha_{a}(\mu)}}+\frac{\sqrt{\gamma_{a}(\mu)}}{\beta_{\operatorname{Br}}(\mu)}\left\|r_{N}^{2}(\cdot ; \mu)\right\|_{Y^{\prime}},
$$

by combining (2.59), (2.62), and (2.61); the bound (2.55) thus follows from (2.63), (2.10), and (2.29), (2.30).

For the error $e_{N}^{p}(\mu)$ in the approximation of the Lagrange multiplier, we again obtain from (2.11) and (2.40) that

$$
\begin{aligned}
\left\|e_{N}^{p}(\mu)\right\|_{Y} & \leq \frac{1}{\beta_{\operatorname{Br}}(\mu)} \sup _{v \in X} \frac{b\left(v, e_{N}^{p}(\mu) ; \mu\right)}{\|v\|_{X}}=\frac{1}{\beta_{\operatorname{Br}}(\mu)} \sup _{v \in X} \frac{r_{N}^{1}(v ; \mu)-a\left(e_{N}^{u}(\mu), v ; \mu\right)}{\|v\|_{X}} \\
& \leq \frac{1}{\beta_{\operatorname{Br}}(\mu)}\left(\left\|r_{N}^{1}(\cdot ; \mu)\right\|_{X^{\prime}}+\sqrt{\gamma_{a}(\mu)}\left\|e_{N}^{u}(\mu)\right\|_{X, \mu}\right)
\end{aligned}
$$

where the last inequality holds by the Cauchy-Schwarz inequality for the inner product $a(\cdot, \cdot ; \mu)$ and (2.8). Together with (2.63) and again (2.29), (2.30), this leads to (2.56).

As it is clearly $\alpha_{a}(\mu) \leq \gamma_{a}(\mu)$, we have $\Delta_{N}^{u, \text { sym }}(\mu)<\Delta_{N}^{u, \operatorname{Br}}(\mu)$ and $\Delta_{N}^{p, \text { sym }}(\mu)<\Delta_{N}^{p, \operatorname{Br}}(\mu)$, which eventually yields (2.57).

Remark 2.4.7. We here comment on the symmetric case in a little more detail.

First, we clearly obtain

$$
\Delta_{N}^{\mathrm{sym}}(\mu) \equiv \sqrt{\left(\Delta_{N}^{u, \text { sym }}(\mu)\right)^{2}+\left(\Delta_{N}^{p, \text { sym }}(\mu)\right)^{2}}, \quad \mu \in \mathcal{D}, N \in \mathbb{N}_{\max }
$$

as a rigorous upper bound for the combined error $e_{N}(\mu)$ such that

$$
\left\|e_{N}(\mu)\right\|_{Z} \leq \Delta_{N}^{\mathrm{sym}}(\mu)<\Delta_{N}^{\mathrm{Br}}(\mu), \quad \forall \mu \in \mathcal{D}, \forall N \in \mathbb{N}_{\max } .
$$

Analogous to Corollary 2.4.3, the associated effectivities then satisfy

$$
1 \leq \eta_{N}^{\mathrm{sym}}(\mu) \equiv \frac{\Delta_{N}^{\mathrm{sym}}(\mu)}{\left\|e_{N}(\mu)\right\|_{Z}} \leq C_{1}^{\mathrm{sym}}(\mu)\left(\gamma_{a}(\mu)+\gamma_{b}(\mu)\right), \quad \forall \mu \in \mathcal{D}, \forall N \in \mathbb{N}_{\max },
$$

where $0<C_{1}^{\mathrm{sym}}(\mu)<C_{1}(\mu)$ is a constant depending on $\alpha_{a}^{\mathrm{LB}}(\mu), \gamma_{a}^{\mathrm{UB}}(\mu)$, and $\beta_{\mathrm{Br}}^{\mathrm{LB}}(\mu)$.

Second, also the error bound (2.52) given in Remark 2.4.4 may be specialized to the symmetric case: Using (2.59), (2.62), (2.61), (2.10), and (2.51), (2.53),

$$
\frac{1}{\alpha_{a}(\mu)} \sup _{v_{0} \in \operatorname{ker}(B(\mu))} \frac{r_{N}^{1}\left(v_{0} ; \mu\right)}{\left\|v_{0}\right\|_{X}}+\sqrt{\frac{\gamma_{a}(\mu)}{\alpha_{a}(\mu)}} \frac{\left\|r_{N}^{2}(\cdot ; \mu)\right\|_{Y^{\prime}}}{\beta_{\mathrm{Br}}(\mu)}
$$

here represents a reliable and efficient a posteriori error estimator for $\left\|e_{N}^{u}(\mu)\right\|_{X}$.

Third, the error $e_{N}^{u}(\mu)$ may clearly also be measured in the energy norm $\|\cdot\|_{X, \mu}=$ $\sqrt{a(\cdot, \cdot ; \mu)}$. For any given $\mu \in \mathcal{D}, N \in \mathbb{N}_{\max }$, and $\alpha_{a}^{\mathrm{LB}}(\mu), \gamma_{a}^{\mathrm{UB}}(\mu), \beta_{\mathrm{Br}}^{\mathrm{LB}}(\mu)$ satisfying $(2.29)$ and $(2.30)$, we define

$$
\tilde{\Delta}_{N}^{u, \operatorname{sym}}(\mu) \equiv \frac{\left\|r_{N}^{1}(\cdot ; \mu)\right\|_{X^{\prime}}}{\sqrt{\alpha_{a}^{\mathrm{LB}}(\mu)}}+\frac{\sqrt{\gamma_{a}^{\mathrm{UB}}(\mu)}}{\beta_{\mathrm{Br}}^{\mathrm{LB}}(\mu)}\left\|r_{N}^{2}(\cdot ; \mu)\right\|_{Y^{\prime}} .
$$


Then, from (2.63) and (2.29), (2.30), $\tilde{\Delta}_{N}^{u, \text { sym }}(\mu)$ is an upper bound for $e_{N}^{u}(\mu)$ such that

$$
\left\|e_{N}^{u}(\mu)\right\|_{X, \mu} \leq \tilde{\Delta}_{N}^{u, \text { sym }}(\mu), \quad \forall \mu \in \mathcal{D}, \forall N \in \mathbb{N}_{\max } .
$$

It directly follows from the proof of Proposition 2.4.6 that the effectivities $\tilde{\eta}_{N}^{u \text {,sym }}(\mu) \equiv$ $\tilde{\Delta}_{N}^{u, \text { sym }}(\mu) /\left\|e_{N}^{u}(\mu)\right\|_{X, \mu}$ and $\eta_{N}^{u, \text { sym }}(\mu) \equiv \Delta_{N}^{u, \text { sym }}(\mu) /\left\|e_{N}^{u}(\mu)\right\|_{X}$ satisfy $\tilde{\eta}_{N}^{u, \text { sym }}(\mu) \leq \eta_{N}^{u, \text { sym }}(\mu)$ for all $\mu \in \mathcal{D}, N \in \mathbb{N}_{\text {max }}$.

In the following, we shall not only examine the above a posteriori error bounds with respect to sharpness and computational efficiency (see §2.6.2 and §3.3.4), but also emphasize their importance to the construction of efficient reduced basis approximation spaces (see §2.7).

\subsection{Construction of Reduced Basis Approximation Spaces}

We now turn to the construction of the reduced basis approximation spaces $X_{N}$ and $Y_{N}$. In general, the reduced basis method constructs its low-dimensional approximation spaces by exploiting the parametric dependence of the problem: Solutions to the truth problem (2.13) reside on the subset

$$
\mathcal{M} \equiv\{(u(\mu), p(\mu)) \mid \mu \in \mathcal{D}\} \subset X \times Y,
$$

and thus the method typically constructs $X_{N} \times Y_{N}, N \in \mathbb{N}_{\max }$, by focusing on $\mathcal{M}$. According to the so-called Lagrange approach, we consider the following option: We assume that we are given a sample of parameter snapshots $\mathcal{D}_{N} \equiv\left\{\mu_{n} \mid 1 \leq n \leq N\right\} \subset \mathcal{D}, N \in \mathbb{N}_{\text {max }}$; for our present purposes, $\mathcal{D}_{N}$ may represent any sequence of nested samples in $\mathcal{D}$, i.e., $\mathcal{D}_{1} \subset \mathcal{D}_{2} \subset \cdots \subset \mathcal{D}_{N}$. For $N \in \mathbb{N}_{\max }$, we then define our reduced basis approximation spaces $X_{N}$ and $Y_{N}$ as

$$
\begin{aligned}
Y_{N} & \equiv \operatorname{span}\left\{p\left(\mu_{n}\right) \mid 1 \leq n \leq N\right\}=\operatorname{span}\left\{\xi_{n} \mid 1 \leq n \leq N\right\}, \\
X_{N}^{0} & \equiv \operatorname{span}\left\{u\left(\mu_{n}\right) \mid 1 \leq n \leq N\right\},
\end{aligned}
$$

where $\xi_{n} \in Y, 1 \leq n \leq N$, denote $(\cdot, \cdot)_{Y}$-orthonormal basis functions; we shall refer to this choice as Option 0.

For saddle point problems, building the reduced basis approximation space solely from snapshots $\left(u\left(\mu_{N}\right), p\left(\mu_{N}\right)\right) \in \mathcal{M}, N \in \mathbb{N}_{\max }$, is not sufficient. We have already seen in $\S 2.3 .2$ that the inf-sup condition (2.17) represents an additional requirement for $X_{N}, Y_{N}$ to provide a well-posed system (2.14). Now, let $\mu$ be an arbitrary but fixed parameter in $\mathcal{D}$. A priori, it is not known whether $\left(X_{N}^{0}, Y_{N}\right)$ satisfies $\beta_{N}(\mu)>0$. More precisely, in case of Option 0 , we meet either of the following two situations:

(i) $\beta_{N}(\mu)=0$ : The system (2.14) is ill-posed as it is not uniquely solvable (see $§ 2.3 .2$ ).

(ii) $\beta_{N}(\mu)>0$ : The system (2.14) is well-posed (see $\S 2.3 .2$ ) and thus uniquely solvable; we obtain approximations $u_{N}(\mu)$ and $p_{N}(\mu)$ for $u(\mu)$ and $p(\mu)$, respectively. However, choosing the approximation spaces $X_{N}, Y_{N}$ as in (2.69), (2.70) is in fact a special case: Whenever $\beta_{N}(\mu)>0$, we in particular have $\operatorname{dim}\left(X_{N}^{0}\right)=\operatorname{dim}\left(Y_{N}\right)$. Consequently, we here encounter a locking phenomenon: Positive inf-sup constants $\beta_{N}(\mu)$ provide a wellposed system (2.14), but the operator $B_{N}(\mu)$ is not only surjective but bijective; the 
space $\operatorname{ker}\left(B_{N}(\mu)\right)$ is thus trivial and there exists only one solution, $B_{N}(\mu)^{-1} g_{N}(\mu)$, satisfying the constraints (2.16). This solution is unlikely to provide an accurate approximation for $u(\mu)$ as components of $u(\mu)$ in $\operatorname{ker}(B(\mu))$ determined by the first equation in (2.13) are essentially neglected.

In summary, Option 0 cannot be expected to provide useful approximations.

We now consider further options to construct the reduced basis approximation spaces that aim to avoid the situations above. First, we address the situation in (i) and thus the issue that the inf-sup constants $\beta_{N}(\mu)$ may approach zero for a given parameter $\mu \in \mathcal{D}$. It is shown in $[92,96]$ that the space $X_{N}^{0}$ can be enriched such that we obtain a provably stable pair $\left(X_{N}, Y_{N}\right)$ where $\beta_{N}(\mu)>0$ holds for all $\mu \in \mathcal{D}$. To explain this in detail, we have to introduce further notations. For $\mu \in \mathcal{D}$ and $1 \leq k \leq Q_{b}$, let $T_{\mu}: Y \rightarrow X$ and $T^{k}: Y \rightarrow X$ be the Riesz representers given by

$$
\left(T_{\mu} q, v\right)_{X}=b(v, q ; \mu), \quad\left(T^{k} q, v\right)_{X}=b^{k}(v, q), \quad \forall v \in X, q \in Y ;
$$

here, $b^{k}(\cdot, \cdot), 1 \leq k \leq Q_{b}$, are the parameter-independent bilinear forms in the affine decomposition $(2.7)$ of $b(\cdot, \cdot ; \mu)$. Since $T_{\mu}$ and $T^{k}$ satisfy

$$
T_{\mu} q=\arg \sup _{v \in X} \frac{b(v, q ; \mu)}{\|v\|_{X}}, \quad T^{k} q=\arg \sup _{v \in X} \frac{b^{k}(v, q)}{\|v\|_{X}}, \quad \forall q \in Y,
$$

for all $\mu \in \mathcal{D}, 1 \leq k \leq Q_{b}$, they are also called supremizer functions. By the affine decomposition $(2.7)$ for $b(\cdot, \cdot ; \mu)$, we in particular have

$$
T_{\mu} q=\sum_{k=1}^{Q_{b}} \Theta_{b}^{k}(\mu) T^{k} q, \quad \forall q \in Y, \quad \forall \mu \in \mathcal{D} .
$$

Now, to any given discrete space $Y_{N} \subseteq Y$ with basis functions $\xi_{n} \in Y, 1 \leq n \leq N_{Y}$, a sufficient condition for $\left(X_{N}, Y_{N}\right)$ to form a stable pair is given by

$$
T_{\mu} \xi_{n} \in X_{N}, \quad \forall 1 \leq n \leq N_{Y}, \quad \forall \mu \in \mathcal{D} ;
$$

in this case, it can be shown (see $[92,96])$ that

$$
\beta_{N}(\mu) \geq \beta_{\mathrm{Br}}(\mu), \quad \forall \mu \in \mathcal{D},
$$

and (2.17) thus follows from the LBB inf-sup condition (2.11). Due to the affine expansion (2.73), (2.74) is satisfied by the finite-dimensional space

$$
\operatorname{span}\left\{T^{k} \xi_{n} \mid 1 \leq n \leq N_{Y}, 1 \leq k \leq Q_{b}\right\} \supseteq\left\{T_{\mu} \xi_{n} \mid 1 \leq n \leq N_{Y}, \mu \in \mathcal{D}\right\} .
$$

Associated to $Y_{N}$ defined as in (2.69), we may therefore choose $X_{N}$ as

$$
X_{N}^{1} \equiv X_{N}^{0} \oplus \operatorname{span}\left\{T^{k} \xi_{n} \mid 1 \leq n \leq N, 1 \leq k \leq Q_{b}\right\},
$$

and obtain by (2.74) and (2.76) pairs of approximation spaces $\left(X_{N}^{1}, Y_{N}\right), N \in \mathbb{N}_{\max }$, which satisfy (2.17) for all parameter values $\mu \in \mathcal{D}$; we shall refer to this choice as Option 1. 
However, Option 1 is also rather expensive as it requires $Q_{b} N$ additional basis functions compared to $X_{N}^{0}$. For this reason, we consider Option 2 as the following modification (see $[88,96])$ : We here add only $N$ supremizer functions instead of $Q_{b} N$ as in (2.77) to $X_{N}^{0}$,

$$
X_{N}^{2} \equiv X_{N}^{0} \oplus \operatorname{span}\left\{T_{\mu_{n}} \xi_{n} \mid 1 \leq n \leq N\right\}
$$

In this case, we can no longer prove stability of $\left(X_{N}^{2}, Y_{N}\right)$ a priori but obtain significantly lower space dimensions than in Option 1.

Now, we focus on the situation described in (ii). The primary issue here is not a vanishing inf-sup constant $\beta_{N}(\mu)$ but rather a trivial space $\operatorname{ker}\left(B_{N}(\mu)\right)$. In Options 1 and 2 , the situation of $\operatorname{ker}(B(\mu))$ being trivial has been avoided by an enrichment of $X_{N}^{0}$ with supremizer functions providing particularly large inf-sup constants $\beta_{N}(\mu)$. For any parameter $\mu \in \mathcal{D}$, it can be expected from (2.52) in Remark 2.4.4 and also the a priori error estimates in $\S 2.3 .3$ that small inf-sup constants $\beta_{N}(\mu)$ will have more dramatic effects on $\left\|e_{N}^{p}(\mu)\right\|_{Y}$ than on $\left\|e_{N}^{u}(\mu)\right\|_{X}$ (see also [13, p. 57, Remark 2.8]): By (2.52) and (2.19), (2.22), $\left\|e_{N}^{u}(\mu)\right\|_{X}$ clearly profits from a large space $\operatorname{ker}\left(B_{N}(\mu)\right)$ but does not explicitly depend on the values of $\beta_{N}(\mu)$. The right-hand side in (2.52) suggests that $\left\|e_{N}^{u}(\mu)\right\|_{X}$ first of all benefits from a good testing space $\operatorname{ker}(B(\mu)) \cap X_{N} \subseteq \operatorname{ker}\left(B_{N}(\mu)\right)$. For this purpose, we now enrich $X_{N}^{0}$ with additional truth solutions $u\left(\mu^{\prime}\right) \in \operatorname{ker}\left(B\left(\mu^{\prime}\right)\right)$ : Given a second sample of parameter snapshots $\mathcal{D}_{N}^{\prime} \equiv\left\{\mu_{n}^{\prime} \mid 1 \leq n \leq N\right\} \subset \mathcal{D}, \mathcal{D}_{N}^{\prime} \cap \mathcal{D}_{N}=\emptyset, N \in \mathbb{N}_{\text {max }}$, we now define Option 3:

$$
X_{N}^{3} \equiv X_{N}^{0} \oplus \operatorname{span}\left\{u\left(\mu_{n}^{\prime}\right) \mid 1 \leq n \leq N\right\}
$$

Again, we cannot prove a priori that this choice provides stable pairs $\left(X_{N}^{3}, Y_{N}\right), N \in \mathbb{N}_{\max }$.

Note that for algebraic stability reasons, we in fact express both $Y_{N}$ and $X_{N}$ by orthonormal basis functions: Analogous to (2.69), we also set $X_{N}=\operatorname{span}\left\{\phi_{m} \mid 1 \leq m \leq N_{X}\right\}$, where $\phi_{m} \in X, 1 \leq m \leq N_{X}$, are $(\cdot, \cdot)_{X}$-orthonormal.

\subsection{Offline-Online Computational Procedure}

The basic strategy lies in the $\mu$-affine dependence (2.7) of the involved operators. Since much of this machinery is by now standard in reduced basis methods (see, e.g., [87, 95]), we only briefly summarize the Offline-Online procedure and associated computational cost.

\subsubsection{Reduced Basis Approximation}

Noting that for any $\mu \in \mathcal{D}$, we can expand $u_{N}(\mu) \in X_{N}$ and $p_{N}(\mu) \in Y_{N}$ into

$$
u_{N}(\mu)=\sum_{m=1}^{N_{X}} u_{N m}(\mu) \phi_{m}, \quad p_{N}(\mu)=\sum_{n=1}^{N_{Y}} p_{N n}(\mu) \xi_{n}
$$

the reduced basis system (2.14) may be written as

$$
\sum_{m=1}^{N_{X}} \sum_{k=1}^{Q_{a}} \Theta_{a}^{k}(\mu) A_{N i m}^{k} u_{N m}(\mu)+\sum_{n=1}^{N_{Y}} \sum_{k=1}^{Q_{b}} \Theta_{b}^{k}(\mu) B_{N n i}^{k} p_{N n}(\mu)=\sum_{k=1}^{Q_{f}} \Theta_{f}^{k}(\mu) f_{N i}^{k},
$$


for all $1 \leq i \leq N_{X}$, and

$$
\sum_{m=1}^{N_{X}} \sum_{k=1}^{Q_{b}} \Theta_{b}^{k}(\mu) B_{N j m}^{k} u_{N m}(\mu)=\sum_{k=1}^{Q_{g}} \Theta_{g}^{k}(\mu) g_{N j}^{k}, \quad \forall 1 \leq j \leq N_{Y} ;
$$

here, the $\mu$-independent quantities are given by

$$
\begin{aligned}
A_{N i j}^{k} & \equiv a^{k}\left(\phi_{j}, \phi_{i}\right), \quad 1 \leq i, j \leq N_{X}, 1 \leq k \leq Q_{a}, \\
B_{N j i}^{k} & \equiv b^{k}\left(\phi_{i}, \xi_{j}\right), \quad 1 \leq i \leq N_{X}, 1 \leq j \leq N_{Y}, 1 \leq k \leq Q_{b}, \\
f_{N i}^{k} & \equiv f^{k}\left(\phi_{i}\right), \quad 1 \leq i \leq N_{X}, 1 \leq k \leq Q_{f}, \\
g_{N j}^{k} & \equiv g^{k}\left(\xi_{j}\right), \quad 1 \leq j \leq N_{Y}, 1 \leq k \leq Q_{g} .
\end{aligned}
$$

Offline, we first form and store all parameter-independent quantities (2.83) at $O\left(\mathcal{N}^{*}\right)$ computational cost and storage. Online, for any given parameter value $\mu \in \mathcal{D}$, we then perform the required sums in (2.81), (2.82) and solve the resulting $N_{Z} \times N_{Z}$ system of linear equations for $\left(u_{N}(\mu), p_{N}(\mu)\right)$ at $O\left(N_{Z}^{3}\right)$ computational cost. The expensive Offline stage, which is performed only once and whose cost depends on the large truth dimension $\mathcal{N}$, thus enables the subsequent very inexpensive Online stage that does not depend on $\mathcal{N}$ but only on the considerably smaller dimension $N_{Z}$ of the reduced basis approximation space.

\subsubsection{A Posteriori Error Bounds}

It is clear that there are two sets of components to the computation of the a posteriori error bounds: the calculation of the residual dual norms (2.31), and the calculation of the required lower and upper bounds (2.29), (2.30) to the coercivity, continuity, and/or inf-sup stability constants. The former is again an application of now standard reduced basis techniques that can be found in, e.g., [95]. The latter is achieved by a successive constraint method (SCM) proposed by Huynh et al. [55]. We here summarize the main parts of the methodology focusing on aspects specific to our saddle point context.

Remark 2.6.1. We note that there exist several approaches for the computation of lower bounds to coercivity and inf-sup stability constants. The applicability of techniques proposed in $[71,87]$ is unfortunately very restricted (e.g., to the case of positive coefficient functions in (2.7)); for problems involving geometry variations, we rely on more general but also more complicated and Offline-expensive approaches. Compared to earlier proposals [79, 97, 106], the SCM in [55] represents a method that (i) is very generally applicable, (ii) performs better, and (iii) is easier to implement. In addition to accurate lower bounds, it also provides upper bounds that are remarkably sharp and thus constitute valuable estimates for the respective coercivity and inf-sup constants. Nevertheless, for inf-sup constants, it still involves notable Offline computations that depend on a $Q^{2}$-term affine parameter expansion (see (2.99)). This issue has been tackled by more recent techniques based on a "natural norm" [53]. However, though well suited for certain problems, the proposed algorithm is in our situation highly cumbersome and involves eigenvalue problems that are much more difficult to solve; also, no upper bounds are provided. 


\section{Dual Norms of the Residuals}

We first exploit the $\mu$-affine expansions (2.7) and (2.80) of the involved operators and the reduced basis approximation, respectively, to express the residuals $r_{N}^{1}(\cdot ; \mu)$ and $r_{N}^{2}(\cdot ; \mu)$ defined in (2.32) and (2.33) as

$$
\begin{aligned}
& r_{N}^{1}(v ; \mu)=\sum_{k=1}^{Q_{f}} \Theta_{f}^{k}(\mu) f^{k}(v)-\sum_{m=1}^{N_{X}} \sum_{k=1}^{Q_{a}} u_{N m}(\mu) \Theta_{a}^{k}(\mu) a^{k}\left(\phi_{m}, v\right) \\
&-\sum_{n=1}^{N_{Y}} \sum_{k=1}^{Q_{b}} p_{N n}(\mu) \Theta_{b}^{k}(\mu) b^{k}\left(v, \xi_{n}\right), \quad \forall v \in X, \\
& r_{N}^{2}(q ; \mu)=\sum_{k=1}^{Q_{g}} \Theta_{g}^{k}(\mu) g^{k}(q)-\sum_{m=1}^{N_{X}} \sum_{k=1}^{Q_{b}} u_{N m}(\mu) \Theta_{b}^{k}(\mu) b^{k}\left(\phi_{m}, q\right), \quad \forall q \in Y .
\end{aligned}
$$

Setting $Q_{1} \equiv Q_{f}+N_{X} Q_{a}+N_{Y} Q_{b}$ and $Q_{2} \equiv Q_{g}+N_{X} Q_{b}$, this can be written succinctly as

$$
\begin{aligned}
& r_{N}^{1}(v ; \mu)=\sum_{k=1}^{Q_{1}} \Theta_{N}^{1, k}(\mu) r_{N}^{1, k}(v), \quad \forall v \in X, \\
& r_{N}^{2}(q ; \mu)=\sum_{k=1}^{Q_{2}} \Theta_{N}^{2, k}(\mu) r_{N}^{2, k}(q), \quad \forall q \in Y,
\end{aligned}
$$

where the coefficient functions $\Theta_{N}^{1, k}(\mu), 1 \leq k \leq Q_{1}$, and $\Theta_{N}^{2, k}(\mu), 1 \leq k \leq Q_{2}$, depend on $\mu$ explicitly through the coefficient functions $\Theta_{\mathcal{I}}^{k}(\mu), 1 \leq k \leq Q_{\mathcal{I}}, \mathcal{I}=a, b, f, g$, but also implicitly through $u_{N}(\mu)$ and $p_{N}(\mu) ; r_{N}^{1, k}(\cdot) \in X^{\prime}, 1 \leq k \leq Q_{1}$, and $r_{N}^{2, k}(\cdot) \in Y^{\prime}, 1 \leq k \leq Q_{2}$, are parameter-independent linear functionals.

We now denote by $\hat{e}_{N}^{1, k} \in X$ and $\hat{e}_{N}^{2, k} \in Y$ the Riesz representers associated with $r_{N}^{1, k}(\cdot)$ and $r_{N}^{2, k}(\cdot)$, respectively, which are obtained as the solutions to the $\mu$-independent linear problems

$$
\begin{aligned}
& \left(\hat{e}_{N}^{1, k}, v\right)_{X}=r_{N}^{1, k}(v), \quad \forall v \in X, \quad \forall 1 \leq k \leq Q_{1}, \\
& \left(\hat{e}_{N}^{2, k}, q\right)_{Y}=r_{N}^{2, k}(q), \quad \forall q \in Y, \quad \forall 1 \leq k \leq Q_{2} .
\end{aligned}
$$

By (2.84) and (2.85), the Riesz representers $\hat{e}_{N}^{1}(\mu) \in X$ and $\hat{e}_{N}^{2}(\mu) \in Y$ associated with the residuals $r_{N}^{1}(\cdot ; \mu)$ and $r_{N}^{2}(\cdot ; \mu)$ are then given by

$$
\hat{e}_{N}^{1}(\mu)=\sum_{k=1}^{Q_{1}} \Theta_{N}^{1, k}(\mu) \hat{e}_{N}^{1, k}, \quad \hat{e}_{N}^{2}(\mu)=\sum_{k=1}^{Q_{2}} \Theta_{N}^{2, k}(\mu) \hat{e}_{N}^{2, k},
$$

and it finally follows that

$$
\begin{gathered}
\left\|r_{N}^{1}(\cdot ; \mu)\right\|_{X^{\prime}}^{2}=\left(\hat{e}_{N}^{1}(\mu), \hat{e}_{N}^{1}(\mu)\right)_{X}=\sum_{k, l=1}^{Q_{1}} \Theta_{N}^{1, k}(\mu) \Theta_{N}^{1, l}(\mu)\left(\hat{e}_{N}^{1, k}, \hat{e}_{N}^{1, l}\right)_{X}, \\
\left\|r_{N}^{2}(\cdot ; \mu)\right\|_{Y^{\prime}}^{2}=\left(\hat{e}_{N}^{2}(\mu), \hat{e}_{N}^{2}(\mu)\right)_{Y}=\sum_{k, l=1}^{Q_{2}} \Theta_{N}^{2, k}(\mu) \Theta_{N}^{2, l}(\mu)\left(\hat{e}_{N}^{2, k}, \hat{e}_{N}^{2, l}\right)_{Y} .
\end{gathered}
$$


We may now summarize the Offline-Online decomposition.

In the Offline stage, we find the Riesz representers $\hat{e}_{N}^{1, k}, 1 \leq k \leq Q_{1}$, and $\hat{e}_{N}^{2, k}, 1 \leq k \leq$ $Q_{2}$, and form the associated inner products $\left(\hat{e}_{N}^{1, k}, \hat{e}_{N}^{1, l}\right)_{X}, 1 \leq k, l \leq Q_{1}$, and $\left(\hat{e}_{N}^{2, k}, \hat{e}_{N}^{2, l}\right)_{Y}$, $1 \leq k, l \leq Q_{2}$. These cost clearly depend on $\mathcal{N}$ : We have to solve $Q_{1}$ and $Q_{2}$ linear problems of type (2.86) and (2.87), respectively, and to compute $\left(Q_{1}\right)^{2}$ and $\left(Q_{2}\right)^{2}$ inner products in $X$ and $Y$. In the Online stage, given the reduced basis coefficients $u_{N m}(\mu)$, $1 \leq m \leq N_{X}$, and $p_{N n}(\mu), 1 \leq n \leq N_{Y}$, we can then readily compute the coefficient functions $\Theta_{N}^{1, k}(\mu), 1 \leq k \leq Q_{1}$, and $\Theta_{N}^{2, k}(\mu), 1 \leq k \leq Q_{2}$. From the stored inner products, we then assemble the sums (2.88) and (2.89) at $O\left(\left(Q_{1}\right)^{2}\right)=O\left(\left(Q_{f}+N_{X} Q_{a}+N_{Y} Q_{b}\right)^{2}\right)$ and $O\left(\left(Q_{2}\right)^{2}\right)=O\left(\left(Q_{g}+N_{X} Q_{b}\right)^{2}\right)$ operations, respectively. As desired, the operation count for the Online stage is thus independent of $\mathcal{N}$.

We now address the second component required for the calculation of the a posteriori error bounds.

\section{Lower and Upper Bounds to Coercivity Constants}

We first discuss the calculation of (Online-)efficient lower and upper bounds (2.29) to the coercivity constants $\alpha_{a}(\mu)$.

Following [55], the coercivity constants $(2.10)$ can be written as the minimum of the functional $J: \mathbb{R}^{Q_{a}} \times \mathcal{D} \rightarrow \mathbb{R}, J(y ; \mu) \equiv \sum_{k=1}^{Q_{a}} \Theta_{a}^{k}(\mu) y_{k}$,

$$
\alpha_{a}(\mu)=\min _{y \in \mathcal{Y}} J(y ; \mu), \quad \forall \mu \in \mathcal{D},
$$

over the set $\mathcal{Y} \equiv\left\{y \in \mathbb{R}^{Q_{a}} \mid \exists v_{y} \in X: y_{k}=a^{k}\left(v_{y}, v_{y}\right) /\left\|v_{y}\right\|_{X}^{2}, 1 \leq k \leq Q_{a}\right\}$; here, $a^{k}(\cdot, \cdot)$, $1 \leq k \leq Q_{a}$, are the parameter-independent bilinear forms in the affine decomposition (2.7). Now, for any $\mu \in \mathcal{D}$, we approximate $\mathcal{Y}$ by sets $\mathcal{Y}_{\mathrm{UB}}, \mathcal{Y}_{\mathrm{LB}} \subseteq \mathbb{R}^{Q_{a}}$ such that

$$
\mathcal{Y}_{\mathrm{UB}} \subseteq \mathcal{Y} \subseteq \mathcal{Y}_{\mathrm{LB}}
$$

and clearly obtain lower and upper bounds to (2.90) by setting

$$
\alpha_{a}^{\mathrm{LB}}(\mu) \equiv \min _{y \in \mathcal{Y}_{\mathrm{LB}}} J(y ; \mu), \quad \alpha_{a}^{\mathrm{UB}}(\mu) \equiv \min _{y \in \mathcal{Y}_{\mathrm{UB}}} J(y ; \mu) .
$$

The sets in (2.91) are constructed as follows: For a given $M_{\alpha} \in \mathbb{N}$ and a sample $C_{K} \equiv\left\{\omega_{i} \mid\right.$ $1 \leq i \leq K\}$ of parameters in $\mathcal{D}$, let $\mathcal{P}_{M_{\alpha}}\left(\mu ; C_{K}\right)$ denote the set of the $M_{\alpha}$ points closest to $\mu$ in the Euclidean norm. For any $\mu \in \mathcal{D}$, we then define

$$
\begin{aligned}
\mathcal{Y}_{\mathrm{LB}}\left(\mu ; C_{K}\right) & \equiv\left\{y \in \mathcal{B} \mid J\left(y ; \mu^{\prime}\right) \geq \alpha_{a}\left(\mu^{\prime}\right), \forall \mu^{\prime} \in \mathcal{P}_{M_{\alpha}}\left(\mu, C_{K}\right)\right\}, \\
\mathcal{Y}_{\mathrm{UB}}\left(C_{K}\right) & \equiv\left\{y^{*}(\omega) \mid \omega \in C_{K}\right\}=\left\{y^{*}\left(\omega_{1}\right), \ldots, y^{*}\left(\omega_{K}\right)\right\},
\end{aligned}
$$

where $\mathcal{B} \equiv \prod_{k=1}^{Q_{a}}\left[b_{k}^{-}, b_{k}^{+}\right] \subseteq \mathbb{R}^{Q_{a}}$ with

$$
b_{k}^{-} \equiv \inf _{v \in X} \frac{a^{k}(v, v)}{\|v\|_{X}^{2}}, \quad b_{k}^{+} \equiv \sup _{v \in X} \frac{a^{k}(v, v)}{\|v\|_{X}^{2}}, \quad \forall 1 \leq k \leq Q_{a},
$$

and $y^{*}(\mu) \in \mathbb{R}^{Q_{a}}$ is given by

$$
y^{*}(\mu)_{k} \equiv \frac{a^{k}\left(v_{\mu}, v_{\mu}\right)}{\left\|v_{\mu}\right\|_{X}^{2}}, \quad \forall 1 \leq k \leq Q_{a}, \quad \text { where } v_{\mu} \equiv \arg \inf _{v \in X} \frac{a(v, v ; \mu)}{\|v\|_{X}^{2}} .
$$


These sets then satisfy (2.91) (see [55]) and thus, $\alpha_{a}^{\mathrm{LB}}(\mu)$ and $\alpha_{a}^{\mathrm{UB}}(\mu)$ defined by $(2.92)$ provide the required lower and upper bounds to $\alpha_{a}(\mu)$.

Now, the idea is to construct the parameter set $C_{K}$ sufficiently large so that for a given $\mu \in \mathcal{D}$, (i) $y^{*}(\mu)$ will be sufficiently close to a member in $\mathcal{Y}_{\mathrm{UB}}\left(C_{K}\right)$ to provide a good upper bound, and (ii) the constraints in $\mathcal{Y}_{\mathrm{LB}}\left(\mu ; C_{K}\right)$ will be sufficiently strict to provide a good lower bound. For this purpose, we build $C_{K}$ by an Offline greedy algorithm: Starting with an exhaustive sample $\Xi$ of parameters in $\mathcal{D}$ and an initial sample $C_{1} \equiv\left\{\omega_{1}\right\}, K=1$, we enrich $C_{K}$ by the parameter for which the ratio (as an estimator for the relative error in the lower and upper bounds)

$$
\frac{\alpha_{a}^{\mathrm{UB}}(\mu)-\alpha_{a}^{\mathrm{LB}}(\mu)}{\alpha_{a}^{\mathrm{UB}}(\mu)}
$$

attains its maximum over $\Xi$. The algorithm stops when (2.97) satisfies a prescribed tolerance $\delta_{\text {tol }}^{\mathrm{SCM}}<1$; we then set $K_{\max }=K$.

For the Offline stage, the notable computations are: (i) $2 Q_{a}$ eigenproblems over $X$ to form $\mathcal{B}$ (see (2.95)), (ii) $K_{\max }$ eigenproblems over $X$ to obtain $\alpha_{a}(\omega)$ and $v_{\omega}$ (see (2.96)) for all $\omega \in C_{K_{\max }}$, (iii) $K_{\max } Q_{a}$ inner products over $X$ to form $\mathcal{Y}_{\mathrm{UB}}\left(C_{K_{\max }}\right)$, and (iv) $|\Xi| K_{\max }$ LP's with $2 Q_{a}+M_{\alpha}$ constraints to compute $\alpha_{a}^{\mathrm{LB}}(\mu)$. For any given parameter $\mu \in \mathcal{D}$, we then obtain $\alpha_{a}^{\mathrm{LB}}(\mu), \alpha_{a}^{\mathrm{UB}}(\mu)$ in a highly efficient Online stage, where notable computations are only a single LP with $2 Q_{a}+M_{\alpha}$ constraints associated with $\alpha_{a}^{\mathrm{LB}}(\mu)$. For the exact choice of $\delta_{\text {tol }}^{\mathrm{SCM}}$ and values for $K_{\max }$ in practice, we refer to $\S 3.3 .4, \S 5.3 .3$, and $\S 6.8$.

\section{Lower and Upper Bounds to Inf-Sup Constants}

As suggested in [55], the calculation of (Online-)efficient lower and upper bounds (2.30) to inf-sup constants can be traced back to the calculation of bounds to coercivity constants.

For the Brezzi inf-sup constants (2.11), we obtain from (2.72) that

$$
\left(\beta_{\mathrm{Br}}(\mu)\right)^{2}=\inf _{q \in Y} \frac{\left(T_{\mu} q, T_{\mu} q\right)_{X}}{\|q\|_{Y}^{2}}, \quad \forall \mu \in \mathcal{D},
$$

and thus $\left(\beta_{\mathrm{Br}}(\mu)\right)^{2}$ represents the coercivity constant of the continuous, symmetric, and coercive (due to (2.11)) bilinear form $\mathrm{B}(\cdot, \cdot ; \mu): Y \times Y \rightarrow \mathbb{R}$ with $\mathrm{B}(p, q ; \mu) \equiv\left(T_{\mu} p, T_{\mu} q\right)_{X}$ for all $p, q \in Y$. From $(2.73), \mathrm{B}(\cdot, \cdot ; \mu)$ can be $\mu$-affinely expanded as

$$
\mathrm{B}(p, q ; \mu)=\left(T_{\mu} p, T_{\mu} q\right)_{X}=\sum_{\mathrm{K}=1}^{\mathrm{Q}} \Theta_{\mathrm{B}}^{\mathrm{K}}(\mu) \mathrm{B}^{\mathrm{K}}(p, q), \quad \forall p, q \in Y, \quad \forall \mu \in \mathcal{D},
$$

where $\mathrm{Q} \equiv Q_{b}\left(Q_{b}+1\right) / 2$, and $\Theta_{\mathrm{B}}^{\mathrm{K}}(\mu), \mathrm{B}^{\mathrm{K}}(\cdot, \cdot)$ are given by

$$
\begin{aligned}
\Theta_{\mathrm{B}}^{\mathrm{K}}(\mu) & =\Theta_{\mathrm{B}}^{(k, l)}(\mu) \equiv\left(\frac{1}{1+\delta_{k l}}\right) \Theta_{b}^{k}(\mu) \Theta_{b}^{l}(\mu), \quad \forall \mu \in \mathcal{D}, \\
\mathrm{B}^{\mathrm{K}}(p, q) & =\mathrm{B}^{(k, l)}(p, q) \equiv\left(T^{k} p, T^{l} q\right)_{X}+\left(T^{l} p, T^{k} q\right)_{X}, \quad \forall p, q \in Y,
\end{aligned}
$$

for all $1 \leq \mathrm{K} \equiv(k, l) \leq \mathrm{Q}, 1 \leq k \leq l \leq Q_{b}$; here, $\delta_{k l}$ denotes the Kronecker delta.

We therefore obtain (Online-)efficient lower and upper bounds to $\left(\beta_{\mathrm{Br}}(\mu)\right)^{2}$ by the method described for coercivity constants. However, we note that the computational cost for the Offline stage here depend on $\mathrm{Q}=O\left(Q_{b}^{2}\right) \geq Q_{b}$. 
An analogous procedure can be applied to compute (Online-)efficient lower and upper bounds $\beta_{\mathrm{Ba}}^{\mathrm{LB}}(\mu), \beta_{\mathrm{Ba}}^{\mathrm{UB}}(\mu)$ to the Babuška inf-sup constants (2.12) (see [55]). It only differs in the computational cost for the Offline stage: They here depend on $Q^{2}$ where $\max \left\{Q_{a}, Q_{b}\right\} \leq$ $Q \leq Q_{a}+Q_{b}$.

\section{Lower and Upper Bounds to Continuity Constants}

For the calculation of (Online-)efficient lower and upper bounds (2.29) to the continuity constants $\gamma_{a}(\mu)$, we distinguish between the symmetric and the nonsymmetric case: If $a(\cdot, \cdot ; \mu)$ is symmetric for all $\mu \in \mathcal{D}$, lower and upper bounds may be computed by an analogous procedure as applied for coercivity constants; the nonsymmetric case is then traced back to the symmetric case by an analogous approach as pursued for inf-sup constants.

The symmetric case: We first consider the case where $a(\cdot, \cdot ; \mu)$ is symmetric for all $\mu \in \mathcal{D}$. The continuity constants (2.8) are then given by

$$
\gamma_{a}(\mu)=\sup _{v \in X} \frac{a(v, v ; \mu)}{\|v\|_{X}^{2}}=\max _{y \in \mathcal{Y}} J(y ; \mu), \quad \forall \mu \in \mathcal{D},
$$

where the functional $J$ and the set $\mathcal{Y}$ are defined as before. Therefore, for any $\mu \in \mathcal{D}$, we here construct sets $\mathcal{Y}_{\mathrm{LB}}\left(C_{K}\right) \subseteq \mathcal{Y} \subseteq \mathcal{Y}_{\mathrm{UB}}\left(\mu ; C_{K}\right)$ by

$$
\begin{aligned}
\mathcal{Y}_{\mathrm{LB}}\left(C_{K}\right) & \equiv\left\{y^{* *}(\omega) \mid \omega \in C_{K}\right\}, \\
\mathcal{Y}_{\mathrm{UB}}\left(\mu ; C_{K}\right) & \equiv\left\{y \in \mathcal{B} \mid J\left(y ; \mu^{\prime}\right) \leq \gamma_{a}\left(\mu^{\prime}\right), \forall \mu^{\prime} \in P_{M_{\alpha}}\left(\mu ; C_{K}\right)\right\},
\end{aligned}
$$

where $y^{* *}(\mu) \in \mathbb{R}^{Q_{a}}$ is defined as

$$
y^{* *}(\mu)_{k} \equiv \frac{a^{k}\left(w_{\mu}, w_{\mu}\right)}{\left\|w_{\mu}\right\|_{X}^{2}}, \quad \forall 1 \leq k \leq Q_{a}, \quad \text { where } w_{\mu} \equiv \arg \sup _{v \in X} \frac{a(v, v ; \mu)}{\|v\|_{X}^{2}} ;
$$

lower and upper bounds to the continuity constants are then obtained by

$$
\gamma_{a}^{\mathrm{LB}}(\mu) \equiv \max _{y \in \mathcal{Y}_{\mathrm{LB}}\left(C_{K}\right)} J(y ; \mu), \quad \gamma_{a}^{\mathrm{UB}}(\mu) \equiv \max _{y \in \mathcal{Y}_{\mathrm{UB}}\left(\mu ; C_{K}\right)} J(y ; \mu) .
$$

We build the set $C_{K}$ by the same greedy algorithm as described for coercivity constants, only that we here consider the ratio

$$
\frac{\gamma_{a}^{\mathrm{UB}}(\mu)-\gamma_{a}^{\mathrm{LB}}(\mu)}{\gamma_{a}^{\mathrm{LB}}(\mu)}
$$

to estimate the error in the bounds; computational cost essentially remain the same as listed for the coercivity constants.

The nonsymmetric case: Similar to (2.98), we obtain for the continuity constants (2.8) that

$$
\left(\gamma_{a}(\mu)\right)^{2}=\sup _{v \in X} \frac{\left(S_{\mu} v, S_{\mu} v\right)_{X}}{\|v\|_{X}^{2}}, \quad \forall \mu \in \mathcal{D},
$$

where $S_{\mu}: X \rightarrow X$ denotes the Riesz representer associated with $a(\cdot, \cdot ; \mu)$ given by

$$
\left(S_{\mu} u, v\right)_{X}=a(u, v ; \mu), \quad \forall v \in X, \forall u \in X, \quad \forall \mu \in \mathcal{D} .
$$


Thus, $\left(\gamma_{a}(\mu)\right)^{2}$ represents the continuity constant of the continuous (due to (2.8)) and symmetric bilinear form $\mathrm{A}(\cdot, \cdot ; \mu): X \times X \rightarrow \mathbb{R}$ with $\mathrm{A}(u, v ; \mu) \equiv\left(S_{\mu} u, S_{\mu} v\right)_{X}$ for all $u, v \in X$, which moreover exhibits a $\mu$-affine expansion analogous to (2.99).

We therefore obtain (Online-)efficient lower and upper bounds to $\left(\gamma_{a}(\mu)\right)^{2}$ by the method described above for the symmetric case. However, as for inf-sup constants, we note that the computational cost for the Offline stage now depend on $Q_{a}^{2} \geq Q_{a}$.

\subsection{Adaptive Sampling Procedures}

In this section, all parts of the methodology discussed in $\S 2.4, \S 2.5$, and $\S 2.6$ coalesce to tackle the key question of how to construct reduced basis approximation spaces providing rapidly convergent approximations.

Rigorous and computationally efficient reduced basis a posteriori error bounds enable us to invoke a greedy sampling process (see $[10,15]$ and the references therein) identifying relatively few basis functions that suffice to warrant a desired accuracy for any parameter query: Starting with an exhaustive sample $\Sigma$ of parameter points in $\mathcal{D}$ and an initial sample $\mu_{1}$, it detects the parameter $\mu_{2}$ for which a chosen error bound $\Delta_{N}(\mu)$ attains its maximum over $\Sigma$ and then enriches the current reduced basis approximation spaces with the new basis functions associated with $\mu_{2}$ (see $\S 2.5$ ); this step is repeated until a prescribed error tolerance is satisfied. In the case of saddle point problems, the inf-sup condition (2.17) represents an additional requirement for the reduced basis approximation spaces. Therefore, according to our observations in $\S 2.5$, the above sampling process will in general (e.g., in combination with Option 0 (see (2.70)) not succeed. In earlier work (see, e.g., [75]), it has been applied in combination with either Option 1 (see (2.77)) or Option 2 (see (2.78)), where stability is guaranteed by adding supremizer functions to the reduced basis approximation space $X_{N}$ for the primal variable in each step. For Option 2, the exact procedure is summarized in Algorithm 1.

However, using this procedure, the question arises of whether the resulting reduced basis approximation spaces are indeed computationally efficient and a reduced basis approximation space $X_{N}$ with a dimension twice as large as for $Y_{N}$ is required to guarantee stability. Addressing this issue, we here present a new adaptive sampling procedure for saddle point problems: In addition to greedily selecting the parameter snapshots $\mu_{1}, \ldots, \mu_{N}$ (see $\S 2.5$ ), we now also adaptively recognize the need for stabilization and thus identify relatively few basis functions that suffice to warrant both a desired accuracy and stability for any parameter query. Note that techniques similar to those presented here have also been proposed in [23] in the context of transport dominated problems.

We assume that we are given a stable pair $\left(X_{N-1}, Y_{N-1}\right)$ of reduced basis approximation spaces that is based on the parameter snapshots $\mathcal{D}_{N-1} \equiv\left\{\mu_{1}, \ldots, \mu_{N-1}\right\} \subset \Sigma$ (see $\S 2.5)$. As in the standard greedy procedure (see Algorithm 1), we use our rigorous and (Online-)efficient error bounds (see $\S 2.4$ and $\S 2.6$ ) to find $\mu_{N} \in \Sigma$ and thus the truth solution $\left(u\left(\mu_{N}\right), p\left(\mu_{N}\right)\right) \in \mathcal{M}$ that is represented least in the current basis; this solution is then appended to the current basis, and we obtain a subsequent pair $\left(X_{N}, Y_{N}\right)$.

From our observations in $\S 2.3 .2, \S 2.3 .3$, and $\S 2.5$, we see that the new pair $\left(X_{N}, Y_{N}\right)$ will in general provide useful approximations only if the inf-sup constants $\beta_{N}(\mu), \mu \in \mathcal{D}$, do not become too small. This can be achieved through an additional enrichment of $X_{N}$ : We may 

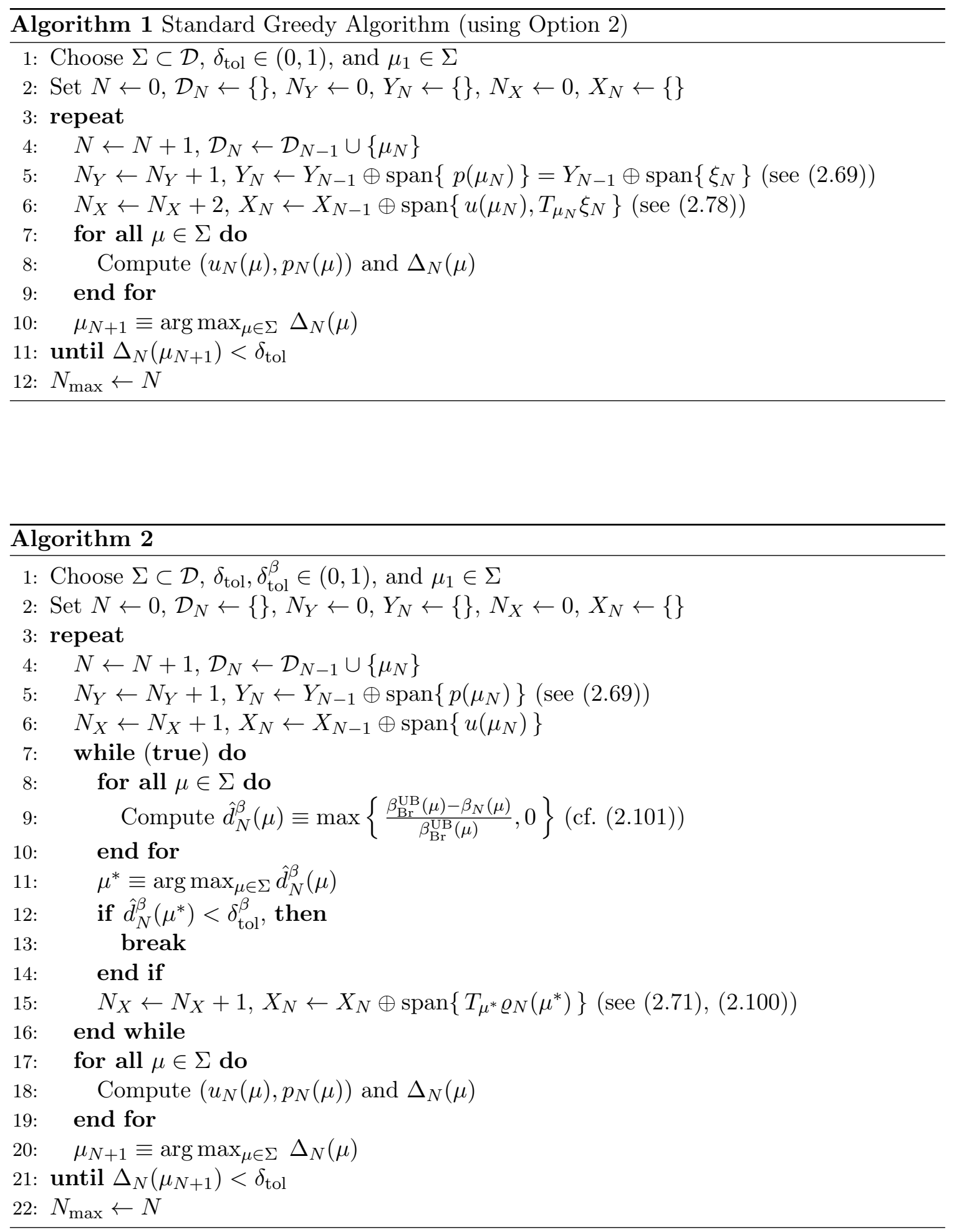


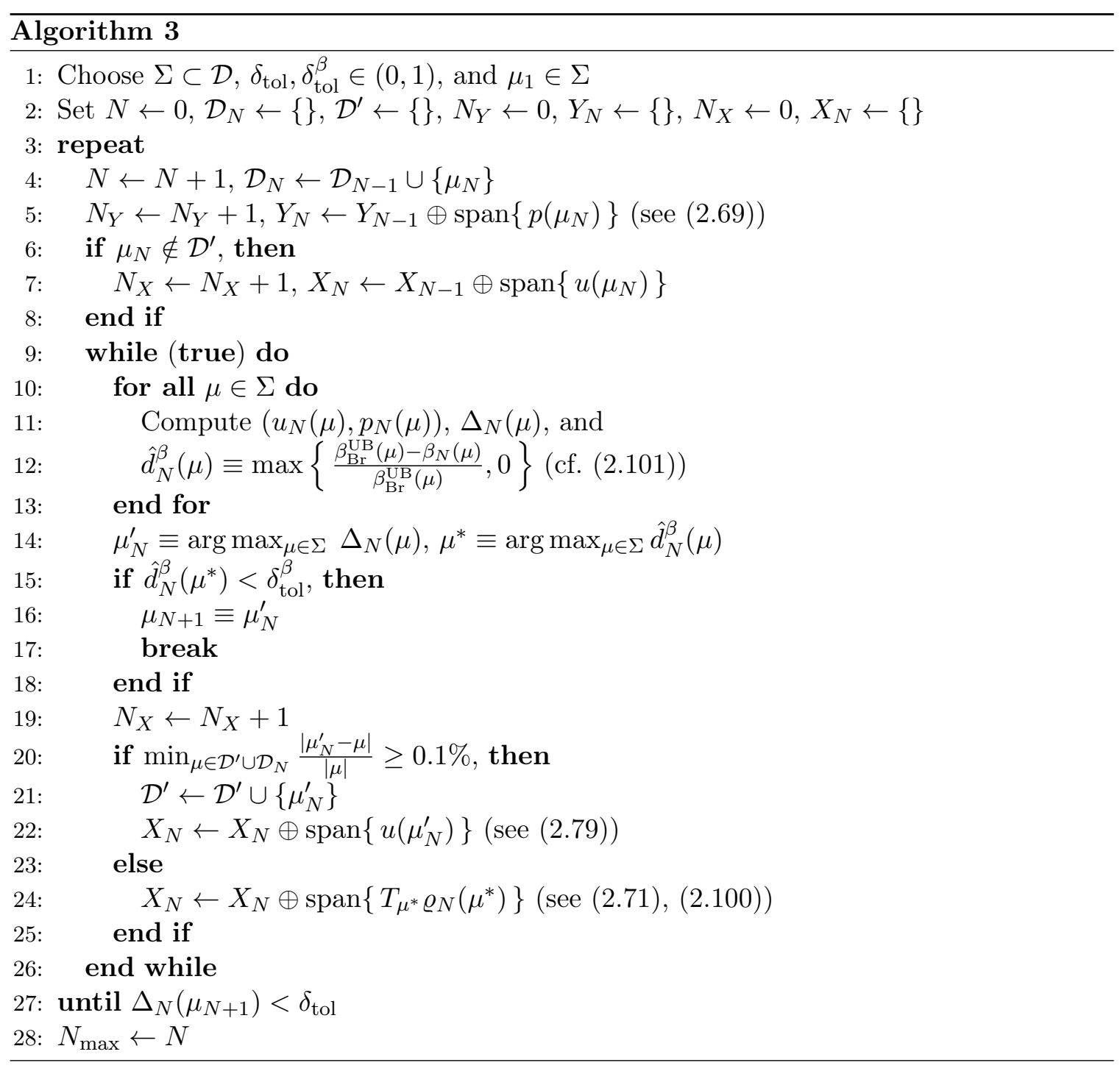


add (i) supremizer functions as in (2.78) or (ii) additional truth solutions as in (2.79). We note from (2.17) that the inf-sup constants $\beta_{N}(\mu)$ may be computed as the solution to a low-dimensional eigenvalue problem; the associated eigenvector is denoted by $\varrho_{N}(\mu) \in Y_{N}$,

$$
\varrho_{N}(\mu) \equiv \arg \inf _{q_{N} \in Y_{N}} \frac{\left\|B_{N}(\mu)^{t} q_{N}\right\|_{X_{N}^{\prime}}}{\left\|q_{N}\right\|_{Y}}, \quad \forall \mu \in \mathcal{D} .
$$

Whether the pair $\left(X_{N}, Y_{N}\right)$ needs to be stabilized is now indicated by the relative distance $d_{N}^{\beta}(\mu)$ between $\beta_{N}(\mu)$ and the truth constant $\beta_{\mathrm{Br}}(\mu)$,

$$
d_{N}^{\beta}(\mu) \equiv \max \left\{\frac{\beta_{\mathrm{Br}}(\mu)-\beta_{N}(\mu)}{\beta_{\mathrm{Br}}(\mu)}, 0\right\}, \quad \forall \mu \in \mathcal{D} .
$$

We choose the parameter $\mu^{*} \in \Sigma$ as the argument that maximizes $d_{N}^{\beta}(\mu)$; whenever $d_{N}^{\beta}\left(\mu^{*}\right)$ exceeds a prescribed tolerance $\delta_{\text {tol }}^{\beta} \in(0,1)$, we additionally enrich the space $X_{N}$.

First, in Algorithm 2, we enrich $X_{N}$ with the supremizer function $T_{\mu^{*}} \varrho_{N}\left(\mu^{*}\right) \in X$ (see (2.71)), $X_{N}^{+} \equiv X_{N} \oplus \operatorname{span}\left\{T_{\mu^{*}} \varrho_{N}\left(\mu^{*}\right)\right\}$. With $X_{N}^{+} \supset X_{N}$, the enriched pair $\left(X_{N}^{+}, Y_{N}\right)$ then clearly satisfies

$$
\beta_{N}^{+}(\mu)=\inf _{q_{N} \in Y_{N}} \sup _{v_{N} \in X_{N}^{+}} \frac{b\left(v_{N}, q_{N} ; \mu\right)}{\left\|q_{N}\right\|_{Y}\left\|v_{N}\right\|_{X}} \geq \inf _{q_{N} \in Y_{N}} \sup _{v_{N} \in X_{N}} \frac{b\left(v_{N}, q_{N} ; \mu\right)}{\left\|q_{N}\right\|_{Y}\left\|v_{N}\right\|_{X}}=\beta_{N}(\mu),
$$

for all $\mu \in \mathcal{D}$. From (2.71), it moreover follows that

$$
\frac{\left\|B_{N}^{+}\left(\mu^{*}\right)^{t} \varrho_{N}\left(\mu^{*}\right)\right\|_{\left(X_{N}^{+}\right)^{\prime}}}{\left\|\varrho_{N}\left(\mu^{*}\right)\right\|_{Y}}=\frac{\left\|B\left(\mu^{*}\right)^{t} \varrho_{N}\left(\mu^{*}\right)\right\|_{X^{\prime}}}{\left\|\varrho_{N}\left(\mu^{*}\right)\right\|_{Y}} \geq \beta_{\mathrm{Br}}\left(\mu^{*}\right),
$$

where the inequality holds by the definition of $\beta_{\mathrm{Br}}(\mu)$ in $(2.11)$. As long as $d_{N}^{\beta,+}\left(\mu^{*}\right)>0$, we therefore obtain $\varrho_{N}^{+}\left(\mu^{*}\right) \neq \varrho_{N}\left(\mu^{*}\right)$ and thus $\beta_{N}^{+}\left(\mu^{*}\right)>\beta_{N}\left(\mu^{*}\right)$ whenever $\beta_{N}\left(\mu^{*}\right)$ is a simple eigenvalue. Second, in Algorithm 3, we aim to stabilize the pair $\left(X_{N}, Y_{N}\right)$ by enriching $X_{N}$ with additional truth solutions $u\left(\mu_{N}^{\prime}\right)$. Only if this is not possible do we resort to the supremizer functions as in Algorithm 2.

\subsection{Concluding Remarks}

We present in this chapter the abstract framework of a new reduced basis approach for saddle point problems. Based on Brezzi's theory [12], we not only derive new rigorous a posteriori bounds for the errors in the reduced basis approximations, but also present a new option to construct stable reduced basis approximation spaces. A new sampling procedure for saddle point problems then assembles computationally efficient reduced basis approximation spaces by recognizing the need for stabilization adaptively.

The developed rigorous reduced basis a posteriori error bounds exhibit significant advantages over existing reduced basis error estimates based on Babuška's theory for general noncoercive problems. First, they do not rely on the highly expensive Offline stage necessary for the efficient Online calculation of lower bounds to the truth Babuška inf-sup constants, 
but instead on much less expensive calculations associated with the truth continuity, coercivity, and Brezzi inf-sup constants. Second, as separate upper bounds for the errors in the approximations of the primal variable and the Lagrange multiplier, they enable the systematic estimation of engineering outputs depending on either of the two.

Symmetric saddle point problems are discussed as a special case in which the a posteriori error bounds may be further sharpened. The analysis presents possible techniques but clearly does not claim to be exhaustive. For example, as current reduced basis techniques allow their exact computation, a posteriori error bounds are here exclusively formulated in terms of the residual dual norms (2.31). However, the symmetric case allows us to consider the dual norm of the residual (2.32) with respect to the energy norm $\|\cdot\|_{X, \mu}=\sqrt{a(\cdot, \cdot ; \mu)}$,

$$
\left\|r_{N}^{1}(\cdot ; \mu)\right\|_{X^{\prime}, \mu} \equiv \sup _{v \in X} \frac{r_{N}^{1}(v ; \mu)}{\|v\|_{X, \mu}}, \quad \forall \mu \in \mathcal{D} .
$$

Using the same techniques as presented in the proof of Proposition 2.4.6, the errors $e_{N}^{u}(\mu)$ and $e_{N}^{p}(\mu)$ in the reduced basis approximations for the primal and Lagrange multiplier variables may then be bounded in terms of $\left\|r_{N}^{1}(\cdot ; \mu)\right\|_{X^{\prime}, \mu},\left\|r_{N}^{2}(\cdot ; \mu)\right\|_{Y^{\prime}}$, and $\tilde{\beta}(\mu)$ (see $(2.60)$ ): For any $\mu \in \mathcal{D}$ and $N \in \mathbb{N}_{\max }$, we can derive that

$$
\begin{aligned}
\left\|e_{N}^{u}(\mu)\right\|_{X, \mu} & \leq\left\|r_{N}^{1}(\cdot ; \mu)\right\|_{X^{\prime}, \mu}+\frac{1}{\tilde{\beta}(\mu)}\left\|r_{N}^{2}(\cdot ; \mu)\right\|_{Y^{\prime}} \\
\left\|e_{N}^{p}(\mu)\right\|_{Y} & \leq \frac{2}{\tilde{\beta}(\mu)}\left\|r_{N}^{1}(\cdot ; \mu)\right\|_{X^{\prime}, \mu}+\frac{1}{(\tilde{\beta}(\mu))^{2}}\left\|r_{N}^{2}(\cdot ; \mu)\right\|_{Y^{\prime}}
\end{aligned}
$$

For these to provide useful error bounds in the reduced basis context, $\left\|r_{N}^{2}(\cdot ; \mu)\right\|_{X^{\prime}, \mu}$ and $\tilde{\beta}(\mu)$ need to be estimated Online-efficiently. Through (2.10) and (2.61), this may be done in terms of $\left\|r_{N}^{1}(\cdot ; \mu)\right\|_{X^{\prime}}, \alpha_{a}(\mu), \gamma_{a}(\mu)$, and $\beta_{\mathrm{Br}}(\mu)$; in this case, (2.102) leads to (2.55) as well and (2.103) results in an upper bound worse than (2.56).

Even though the reduced basis method is based on the affine decomposition (2.7) of the involved operators, problems with nonaffine parameter dependencies can also be handled efficiently: In this case, techniques as proposed in [7, 40] may be applied to recover the setting of $\S 2.2$. 



\section{Chapter 3}

\section{Approximation of the Stokes Equations}

\subsection{Introduction}

In this chapter, we apply the reduced basis methodology developed in Chapter 2 to a Stokes flow in a parametrized domain.

Though often performed as a step to the more general Navier-Stokes equations, the analysis of the Stokes equations remains relevant in many engineering applications. In the field of microfluidics (see, e.g., $[14,50]$ ), fluids are often geometrically constrained to a submillimeter scale; for example, lab-on-a-chip devices deal with the handling of extremely small fluid volumes down to less than pico liters. At these scales, flows often exhibit Reynolds numbers that are small enough for inertial effects to be irrelevant and they can therefore be described by the Stokes equations (see $\S 1.4 .2$ ).

The chapter is organized as follows: In $\S 3.2$, we introduce our Stokes model problem. Motivated by applications in the field of microfluidics, we consider a Stokes flow in a twodimensional microchannel with a parametrized rectangular obstacle. We shall state the strong as well as the weak formulation of the problem and describe the mapping procedure that eventually yields the formulation of a parametrized saddle point problem as introduced in $\S 2.2$. In $\S 3.3$, numerical results then demonstrate: (i) the need to appropriately enrich the reduced basis approximation space for the primal variable; (ii) the significant effects of different enrichment stategies; (iii) the rapid convergence of (stable) reduced basis approximations; and (iv) the advantages of the proposed reduced basis a posteriori error bounds with respect to sharpness and computational cost. Finally, in $§ 3.4$, we give some concluding remarks.

\subsection{Model Problem}

Many microfluidic devices with applications in chemistry, biology, or medicine require the mixing and dispersion of reagents (see, e.g., $[98,99])$. An important area in microfluidic research is therefore concerned with the design of so-called micromixers [18] enhancing the mixing between adjacent streams in a straight, smooth-walled microchannel. Due to the low value of the Reynolds number, turbulence is uncommon with the consequence that mixing is difficult: In the expected laminar flow, mixing occurs by diffusion alone, which can result in very long mixing times. This is clearly unacceptable whenever complete and rapid mixing 


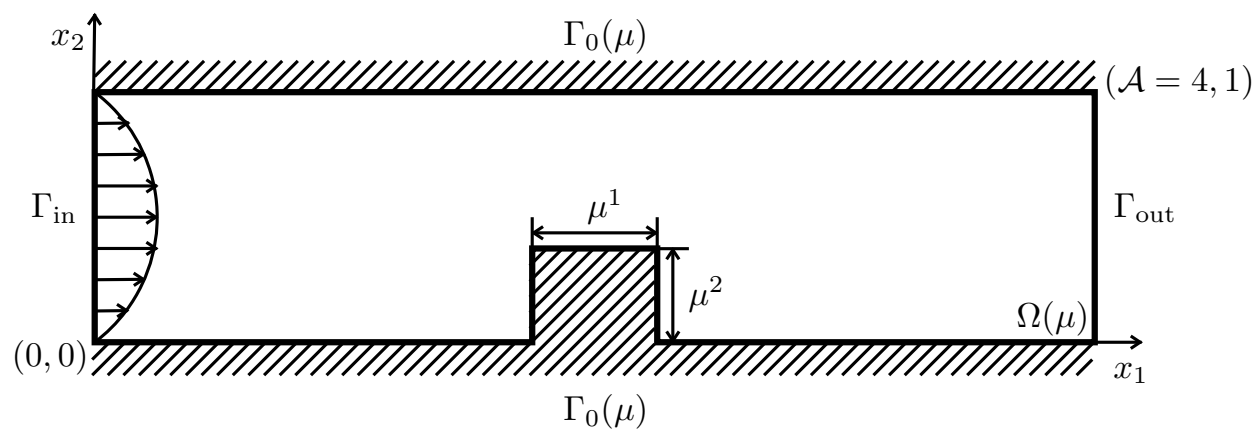

Figure 3.1.: Physical domain $\tilde{\Omega}=\Omega(\mu)$ : a parametrized two-dimensional microchannel with an obstacle. The parameters of interest are width $\mu^{1}$ and height $\mu^{2}$ of the obstacle.

is desired, as required for the initiation of a chemical reaction. To decrease mixing time as well as mixing length, transverse flows must be generated. In this context, there are two general strategies (see [99]): active methods, where transverse flows are generated by local, oscillatory forcing within the channel; and passive methods, where transverse flows result from the interaction of the externally driven flow with the fixed channel geometry.

Our model problem is motivated by questions appearing in the design of passive micromixers. The challenge here is to generate laminar chaos in an accessible geometry, and a simple design that satisfies this criterion uses obstructions or grooves on one wall of the channel to generate transverse components in steady flows (see [99] and the references therein). Questions of interest then address the exact placing and design of such obstructions. We therefore assume the following simplified setting: We consider a Stokes or creeping flow in a two-dimensional channel with a rectangular obstacle as illustrated in Fig. 3.1. The problem depends on two geometric parameters $\mu \equiv\left(\mu^{1}, \mu^{2}\right)$ representing width $\mu^{1}$ and height $\mu^{2}$ of the obstacle $\mathcal{O}(\mu) \equiv\left[\left(\mathcal{A}-\mu^{1}\right) / 2,\left(\mathcal{A}+\mu^{1}\right) / 2\right] \times\left[0, \mu^{2}\right]$, which are assumed to be varying in the prescribed compact parameter set $\mathcal{D} \equiv[0.1,0.5]^{2} \subset \mathbb{R}^{2}$. The channel has a fixed aspect ratio, $\mathcal{A} \equiv 4$. The physical domain is thus $\tilde{\Omega} \equiv \Omega(\mu) \equiv((0, \mathcal{A}) \times(0,1)) \backslash \mathcal{O}(\mu)$ with its boundary $\tilde{\Gamma} \equiv \Gamma(\mu)$. We further assume fully developed flow conditions with a parabolic velocity profile on the inflow boundary $\Gamma_{\text {in }} \equiv\{0\} \times[0,1]$, natural outflow conditions on $\Gamma_{\text {out }} \equiv\{4\} \times[0,1]$, and no-penetration and no-slip velocity conditions on the channel walls and obstacle boundary $\tilde{\Gamma}_{0} \equiv \Gamma_{0}(\mu) \equiv \Gamma(\mu) \backslash\left(\Gamma_{\text {in }} \cup \Gamma_{\text {out }}\right)$.

\subsubsection{Strong Formulation}

For the physical domain $\tilde{\Omega}, \mu \in \mathcal{D}$, we now seek to find the (inhomogeneous) velocity $\tilde{u}_{\mathrm{e}, \mathrm{inh}}: \tilde{\Omega} \rightarrow \mathbb{R}^{2}$ and the pressure $\tilde{p}_{\mathrm{e}}: \tilde{\Omega} \rightarrow \mathbb{R}$ such that

$$
\begin{aligned}
-\tilde{\Delta} \tilde{u}_{\mathrm{e}, \text { inh }}+\tilde{\nabla} \tilde{p}_{\mathrm{e}}=0 & \text { in } \tilde{\Omega}, \\
\tilde{\nabla} \cdot \tilde{u}_{\mathrm{e}, \text { inh }}=0 & \text { in } \tilde{\Omega},
\end{aligned}
$$

with boundary conditions

$$
\tilde{u}_{\mathrm{e}, \text { inh }}=h \quad \text { on } \Gamma_{\mathrm{in}}, \quad \tilde{u}_{\mathrm{e}, \mathrm{inh}}=0 \quad \text { on } \tilde{\Gamma}_{0}, \quad \frac{\partial \tilde{u}_{\mathrm{e}, \text { inh }}}{\partial \tilde{n}}=\tilde{p}_{\mathrm{e}} \tilde{n} \quad \text { on } \tilde{\Gamma}_{\text {out }} ;
$$


here, $\tilde{\Delta}$ and $\tilde{\nabla}$ are the Laplacian and gradient operator over the physical domain $\tilde{\Omega}$ (see Appendix A), respectively, $\tilde{n}$ denotes the unit outward normal, and $h: \mathbb{R}^{2} \rightarrow \mathbb{R}^{2}$ is given by $h(x)=h\left(x_{1}, x_{2}\right) \equiv\left(4 x_{2}\left(1-x_{2}\right), 0\right)$ for all $x=\left(x_{1}, x_{2}\right) \in \mathbb{R}^{2}$.

\subsubsection{Weak Formulation}

We first introduce the function spaces

$$
\begin{aligned}
\tilde{X}_{\mathrm{e}, \text { inh }} & \equiv\left\{\tilde{v} \in\left(H^{1}(\tilde{\Omega})\right)^{2}|\tilde{v}|_{\Gamma_{\mathrm{in}}}=h,\left.\tilde{v}\right|_{\tilde{\Gamma}_{0}}=0\right\}, \\
\tilde{X}_{\mathrm{e}} & \equiv\left\{\tilde{v} \in\left(H^{1}(\tilde{\Omega})\right)^{2}|\tilde{v}|_{\Gamma_{\mathrm{in}} \cup \tilde{\Gamma}_{0}}=0\right\}, \\
\tilde{Y}_{\mathrm{e}} & \equiv L^{2}(\tilde{\Omega}),
\end{aligned}
$$

where $H^{1}(\tilde{\Omega})=\left\{\tilde{v} \in L^{2}(\tilde{\Omega}) \mid \tilde{\nabla} \tilde{v} \in\left(L^{2}(\tilde{\Omega})\right)^{2}\right\}$, and $L^{2}(\tilde{\Omega})$ is the space of square integrable functions over $\tilde{\Omega}$; for more details on Sobolev spaces, we refer to [1]. We then consider the following weak form of the problem (3.1)-(3.3): We seek to find $\tilde{u}_{\mathrm{e}, \text { inh }} \in \tilde{X}_{\mathrm{e}, \text { inh }}$ and $\tilde{p}_{\mathrm{e}} \in \tilde{Y}_{\mathrm{e}}$ such that

$$
\begin{aligned}
\tilde{a}\left(\tilde{u}_{\mathrm{e}, \mathrm{inh}}, \tilde{v}\right)+\tilde{b}\left(\tilde{v}, \tilde{p}_{\mathrm{e}}\right)=0, \quad \forall \tilde{v} \in \tilde{X}_{\mathrm{e}} \\
\tilde{b}\left(\tilde{u}_{\mathrm{e}, \mathrm{inh}}, \tilde{q}\right)=0, \quad \forall \tilde{q} \in \tilde{Y}_{\mathrm{e}},
\end{aligned}
$$

where the bilinear forms $\tilde{a}:\left(H^{1}(\tilde{\Omega})\right)^{2} \times\left(H^{1}(\tilde{\Omega})\right)^{2} \rightarrow \mathbb{R}$ and $\tilde{b}:\left(H^{1}(\tilde{\Omega})\right)^{2} \times \tilde{Y}_{\mathrm{e}} \rightarrow \mathbb{R}$ are defined as

$$
\begin{aligned}
\tilde{a}(\tilde{u}, \tilde{v}) & \equiv \int_{\tilde{\Omega}} \frac{\partial \tilde{u}_{i}}{\partial \tilde{x}_{j}} \frac{\partial \tilde{v}_{i}}{\partial \tilde{x}_{j}} d \tilde{x}, \quad \forall \tilde{u}, \tilde{v} \in\left(H^{1}(\tilde{\Omega})\right)^{2}, \\
\tilde{b}(\tilde{v}, \tilde{q}) & \equiv-\int_{\tilde{\Omega}} \tilde{q} \frac{\partial \tilde{v}_{i}}{\partial \tilde{x}_{i}} d \tilde{x}, \quad \forall \tilde{v} \in\left(H^{1}(\tilde{\Omega})\right)^{2}, \tilde{q} \in \tilde{Y}_{\mathrm{e}} ;
\end{aligned}
$$

here, repeated indices imply summation.

\subsubsection{Formulation as a Parametrized Saddle Point Problem}

We now introduce the lifting function $\tilde{u}_{\mathrm{L}} \in \tilde{X}_{\mathrm{e}, \text { inh }}$ where

$$
\tilde{u}_{\mathrm{L}}\left(\tilde{x}_{1}, \tilde{x}_{2}\right) \equiv \begin{cases}\left(4 \tilde{x}_{2}\left(1-\tilde{x}_{2}\right)\left(1-\tilde{x}_{1}\right), 0\right), & \text { in } \Omega_{\mathrm{L}} \equiv[0,1]^{2}, \\ (0,0), & \text { in } \tilde{\Omega} \backslash \Omega_{\mathrm{L}} .\end{cases}
$$

Note that $\Omega_{\mathrm{L}}$ does not include the $\mu$-dependent part of the domain and $\tilde{u}_{\mathrm{L}}$ is therefore by construction parameter-independent. The inhomogeneous velocity solution $\tilde{u}_{\mathrm{e}, \text { inh }}$ may then be decomposed into $\tilde{u}_{\mathrm{e}, \text { inh }}=\tilde{u}_{\mathrm{L}}+\tilde{u}_{\mathrm{e}}$, where $\tilde{u}_{\mathrm{e}}: \tilde{\Omega} \rightarrow \mathbb{R}^{2}$ satisfies homogeneous Dirichlet boundary conditions on $\Gamma_{\text {in }} \cup \tilde{\Gamma}_{0}$. The governing equations for the (homogeneous) velocity $\tilde{u}_{\mathrm{e}}$ and the pressure $\tilde{p}_{\mathrm{e}}$ are then: We find $\tilde{u}_{\mathrm{e}} \in \tilde{X}_{\mathrm{e}}$ and $\tilde{p}_{\mathrm{e}} \in \tilde{Y}_{\mathrm{e}}$ such that

$$
\begin{aligned}
\tilde{a}\left(\tilde{u}_{\mathrm{e}}, \tilde{v}\right)+\tilde{b}\left(\tilde{v}, \tilde{p}_{\mathrm{e}}\right) & =\tilde{f}(\tilde{v}), \quad \forall \tilde{v} \in \tilde{X}_{\mathrm{e}} \\
\tilde{b}\left(\tilde{u}_{\mathrm{e}}, \tilde{q}\right) & =\tilde{g}(\tilde{q}), \quad \forall \tilde{q} \in \tilde{Y}_{\mathrm{e}}
\end{aligned}
$$

where the right-hand side is given by $\tilde{f} \equiv-\tilde{a}\left(\tilde{u}_{\mathrm{L}}, \cdot\right) \in \tilde{X}_{\mathrm{e}}^{\prime}$ and $\tilde{g} \equiv-\tilde{b}\left(\tilde{u}_{\mathrm{L}}, \cdot\right) \in \tilde{Y}_{\mathrm{e}}^{\prime}$. 


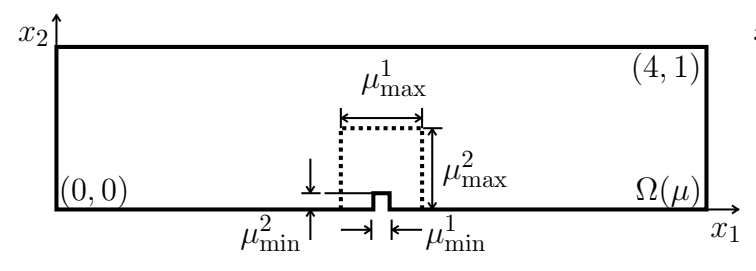

(a)

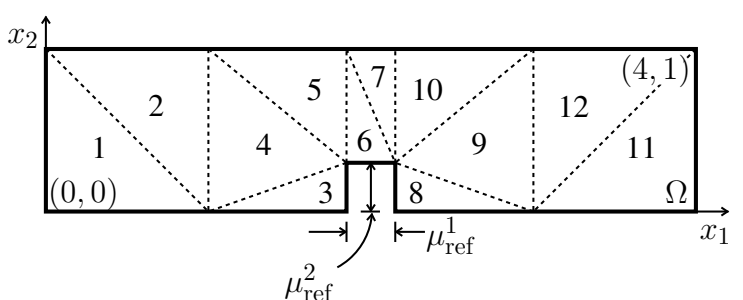

(b)

Figure 3.2.: (a) Parametrized domain $\Omega(\mu)$ for $\mu=\mu_{\min }=(0.1,0.1)$ (solid line) and $\mu=$ $\mu_{\max }=(0.5,0.5)$ (dotted line); (b) reference domain $\Omega$ with $\mu_{\text {ref }}=\left(\mu_{\text {ref }}^{1}, \mu_{\text {ref }}^{2}\right)=$ $(0.3,0.3)$, and corresponding decomposition $\bar{\Omega}=\bigcup_{s=1}^{S} \overline{\Omega^{s}}, S=12$ (see (3.6)).

To finally obtain a parametrized saddle point problem as defined in $\S 2.2 .1$, we now transform the problem statement (3.5) to an equivalent problem posed over a parameterindependent reference domain $\Omega$. To this end, $\tilde{\Omega}=\Omega(\mu)$ is traced back to $\Omega \equiv \Omega\left(\mu_{\text {ref }}\right)$ by a continuous, piecewise affine mapping; for our model problem, we choose $\mu_{\text {ref }} \equiv(0.3,0.3) \in \mathcal{D}$. The boundary of $\Omega$ is then denoted by $\Gamma \equiv \Gamma_{0} \cup \Gamma_{\text {in }} \cup \Gamma_{\text {out }}$ where $\Gamma_{0} \equiv \Gamma_{0}\left(\mu_{\text {ref }}\right)$. For all $\mu \in \mathcal{D}$, the physical domain $\tilde{\Omega}=\Omega(\mu)$ is decomposed into $S \in \mathbb{N}$ subdomains $\tilde{\Omega}^{s}=\Omega^{s}(\mu)$, $1 \leq s \leq S$,

$$
\overline{\tilde{\Omega}}=\bigcup_{s=1}^{S} \overline{\tilde{\Omega}^{s}}, \quad \text { such that } \tilde{\Omega}^{s} \cap \tilde{\Omega}^{r}=\emptyset, \quad \forall 1 \leq s<r \leq S ;
$$

for our model problem, this is demonstrated in Fig. 3.2. We in particular obtain

$$
\bar{\Omega}=\bigcup_{s=1}^{S} \overline{\Omega^{s}}, \quad \text { where } \Omega^{s} \equiv \Omega^{s}\left(\mu_{\text {ref }}\right) \text { and } \Omega^{s} \cap \Omega^{r}=\emptyset, \quad \forall 1 \leq s<r \leq S,
$$

and for any $\mu \in \mathcal{D}, 1 \leq s \leq S$, the subdomain $\tilde{\Omega}^{s}$ may be traced back to $\Omega^{s}$ by an affine coordinate transformation $\tilde{\Phi}^{s} \equiv \Phi^{s}(\cdot ; \mu): \tilde{\Omega}^{s} \rightarrow \Omega^{s}, \Phi^{s}(\tilde{x} ; \mu) \equiv A^{s}(\mu) \tilde{x}+b^{s}(\mu)$, where $A^{s}(\mu) \in \mathbb{R}^{2 \times 2}$ nonsingular and $b^{s}(\mu) \in \mathbb{R}^{2}$. A more detailed discussion of affine geometry transformations can be found in [95].

We then apply standard techniques to transform the problem statement (3.5) to an equivalent problem statement formulated over the reference domain $\Omega$. We denote the function spaces associated with $\Omega$ by $X_{\mathrm{e}}$ and $Y_{\mathrm{e}}$, respectively. On these spaces, we consider the inner products and norms

$$
\begin{aligned}
(u, v)_{X_{\mathrm{e}}} & \equiv \int_{\Omega} \frac{\partial u_{i}}{\partial x_{j}} \frac{\partial v_{i}}{\partial x_{j}} d x, \quad \forall u, v \in X_{\mathrm{e}}, \quad\|\cdot\|_{X_{\mathrm{e}}}=\sqrt{(\cdot, \cdot)_{X_{\mathrm{e}}}} \\
(p, q)_{Y_{\mathrm{e}}} & \equiv \int_{\Omega} p q d x, \quad \forall p, q \in Y_{\mathrm{e}}, \quad\|\cdot\|_{Y_{\mathrm{e}}}=\sqrt{(\cdot, \cdot)_{Y_{\mathrm{e}}}}
\end{aligned}
$$

By setting $v(x) \equiv \tilde{v}(\tilde{x})$ and $q(x) \equiv \tilde{q}(\tilde{x})$ for all $x \in \Omega$, we particularly obtain isomorphisms $\tilde{X}_{\mathrm{e}} \rightarrow X_{\mathrm{e}}, \tilde{v} \mapsto v$, and $\tilde{Y}_{\mathrm{e}} \rightarrow Y_{\mathrm{e}}, \tilde{q} \mapsto q$ (see, e.g., [1]). For any $\mu \in \mathcal{D}$, the problem (3.5) is thus equivalent to: Find $u_{\mathrm{e}}(\mu) \in X_{\mathrm{e}}$ and $p_{\mathrm{e}}(\mu) \in Y_{\mathrm{e}}$ such that

$$
\begin{aligned}
a\left(u_{\mathrm{e}}(\mu), v ; \mu\right)+b\left(v, p_{\mathrm{e}}(\mu) ; \mu\right) & =f(v ; \mu), \quad \forall v \in X_{\mathrm{e}}, \\
b\left(u_{\mathrm{e}}(\mu), q ; \mu\right) & =g(q ; \mu), \quad \forall q \in Y_{\mathrm{e}},
\end{aligned}
$$


where the bilinear and linear forms are given by

$$
\begin{gathered}
a(u, v ; \mu)=\sum_{s=1}^{S} \frac{A_{k j}^{s}(\mu) A_{l j}^{s}(\mu)}{\left|\operatorname{det}\left(A^{s}(\mu)\right)\right|} \int_{\Omega^{s}} \frac{\partial u_{i}}{\partial x_{k}} \frac{\partial v_{i}}{\partial x_{l}} d x, \quad \forall u, v \in X_{\mathrm{e}}, \\
b(v, q ; \mu)=-\sum_{s=1}^{S} \frac{A_{j i}^{s}(\mu)}{\left|\operatorname{det}\left(A^{s}(\mu)\right)\right|} \int_{\Omega^{s}} q \frac{\partial v_{i}}{\partial x_{j}} d x, \quad \forall v \in X_{\mathrm{e}}, q \in Y_{\mathrm{e}}, \\
f(v ; \mu)=f(v)=-\int_{\Omega_{\mathrm{L}}} \frac{\partial u_{\mathrm{L} i}}{\partial x_{j}} \frac{\partial v_{i}}{\partial x_{j}} d x, \quad \forall v \in X_{\mathrm{e}}, \\
g(q ; \mu)=g(q)=\int_{\Omega_{\mathrm{L}}} q \frac{\partial u_{\mathrm{L} i}}{\partial x_{i}} d x, \quad \forall q \in Y_{\mathrm{e}} .
\end{gathered}
$$

The bilinear forms $a(\cdot, \cdot ; \mu)$ and $b(\cdot, \cdot ; \mu)$ now satisfy all the assumptions (2.1), (2.2), (2.3), (2.4), and (2.7) (see, e.g., $[13,36,101])$, and we find ourselves exactly in the setting introduced in $\S 2.2 .1$. We particularly have $Q_{a}=10, Q_{b}=6$, and $Q_{f}=Q_{g}=1$ in the respective $\mu$-affine expansions (2.7).

We choose the truth approximation spaces $X$ and $Y$ in $\S 2.2 .2$ as the standard conforming $\mathbb{P}_{2}-\mathbb{P}_{1}$ (quadratic-linear) Taylor-Hood finite element approximation subspaces [100] over a regular triangulation $\mathcal{T}_{\Omega}$ of $\Omega$. It is a well-known result (see, e.g., $[13,27,36,89]$ ) that in this case, $X$ and $Y$ indeed satisfy the LBB inf-sup condition (2.11).

\subsection{Numerical Results}

We now apply the reduced basis methodology developed in $§ 2.3-\$ 2.7$ to our model problem. In this section, all numerical results are attained using the open source software rb00mit [65], an implementation of the reduced basis framework within the $\mathrm{C}++$ parallel finite element library libMesh [62].

\subsubsection{Truth Approximation}

Based on a fine mesh with 16,602 elements, the truth system (2.13) has a dimension of $\mathcal{N}=72,076$. Figure 3.3 shows the velocity streamlines for three different parameter values

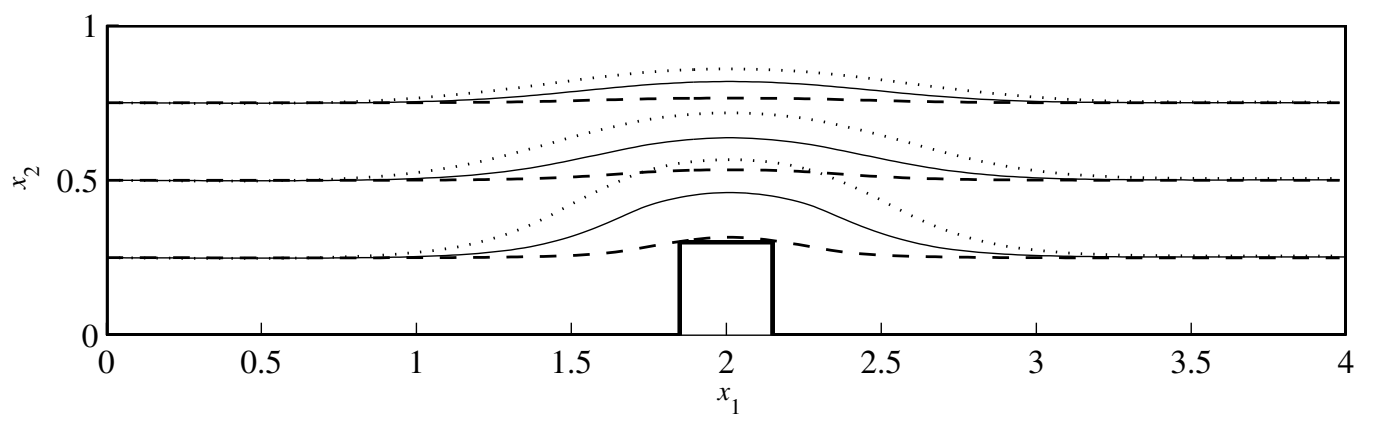

Figure 3.3.: Velocity streamlines for $\mu=(0.1,0.1)$ (dashed), $\mu=(0.3,0.3)$ (solid), and $\mu=(0.5,0.5)$ (dotted) plotted on the reference domain $\Omega$. 
$\mu=(0.1,0.1), \mu=(0.3,0.3)$, and $\mu=(0.5,0.5)$. We observe that the $\mu$-dependence over our parameter domain is clearly nontrivial.

\subsubsection{Reduced Basis Approximation Spaces}

We here present numerical results for the different options introduced in $\S 2.5$ to construct the reduced basis approximation spaces $X_{N}$ and $Y_{N}$. For this purpose, the parameter samples $\mathcal{D}_{N}$ and $\mathcal{D}_{N}^{\prime}$ (see $\left.\S 2.5\right)$ here have been chosen randomly.

First, we demonstrate in practice that a reduced basis approximation space constructed by using Option 0 (see (2.70)) may indeed not provide useful approximations. For our model problem, we obtain values for the inf-sup constants $\beta_{N}(\mu)$ that are small but still positive (see Fig. 3.4(a)); the system (2.14) is uniquely solvable, and we obtain approximations $u_{N}(\mu)$ and $p_{N}(\mu)$ for $u(\mu)$ and $p(\mu)$, respectively. We thus find ourselves in the situation (ii) described in $\S 2.5$ and indeed observe the predicted locking phenomenon: There exists only one solution, $B_{N}(\mu)^{-1} g_{N}(\mu)$, satisfying the constraints (2.16) (see Fig. 3.4(b)). Consequently, as we increase $N$, values of the residual dual norm $\left\|r_{N}^{2}(\cdot ; \mu)\right\|_{Y^{\prime}}$ associated with the constraints become relatively small and negligible in comparison to large values of $\left\|r_{N}^{1}(\cdot ; \mu)\right\|_{X^{\prime}}$ (see Fig. 3.5(a)); our approximations $u_{N}(\mu)$ do not converge satisfactorily, and thus, as suggested by $(2.46)$, neither does $p_{N}(\mu)$ (see Fig. 3.5(b)).

Constructing the reduced basis approximation spaces $X_{N}, Y_{N}$ via Option 1 (see (2.77)), Table 3.1(a) contains values of the inf-sup constants $\beta_{N}(\mu)$ for several parameter values $\mu \in \mathcal{D}$ : We clearly see that they are essentially constant with respect to $N$ and indeed satisfy (2.75). Table 3.1(b) contains the same data when using Option 2 (see (2.78)): We observe that the values of the inf-sup constants $\beta_{N}(\mu)$ here differ only insignificantly from the values obtained by using Option 1; they are also essentially constant in $N$ and satisfy (2.75). In practice, Option 2 thus provides stable approximation spaces satisfying (2.75) at a fraction of the computational cost of Option 1 (see Fig. 3.6).

(a) Option 1

\begin{tabular}{c|c|c|c|c|c|c}
\hline$\mu$ & $\beta_{\mathrm{Br}}(\mu)$ & $\beta_{5}(\mu)$ & $\beta_{10}(\mu)$ & $\beta_{20}(\mu)$ & $\beta_{30}(\mu)$ & $\beta_{40}(\mu)$ \\
\hline$(0.1,0.1)$ & $1.073 \cdot 10^{-1}$ & $1.138 \cdot 10^{-1}$ & $1.137 \cdot 10^{-1}$ & $1.135 \cdot 10^{-1}$ & $1.135 \cdot 10^{-1}$ & $1.135 \cdot 10^{-1}$ \\
$(0.5,0.1)$ & $1.070 \cdot 10^{-1}$ & $1.134 \cdot 10^{-1}$ & $1.133 \cdot 10^{-1}$ & $1.132 \cdot 10^{-1}$ & $1.132 \cdot 10^{-1}$ & $1.131 \cdot 10^{-1}$ \\
$(0.3,0.3)$ & $9.583 \cdot 10^{-2}$ & $9.964 \cdot 10^{-2}$ & $9.963 \cdot 10^{-2}$ & $9.961 \cdot 10^{-2}$ & $9.961 \cdot 10^{-2}$ & $9.961 \cdot 10^{-2}$ \\
$(0.1,0.5)$ & $7.881 \cdot 10^{-2}$ & $8.097 \cdot 10^{-2}$ & $8.088 \cdot 10^{-2}$ & $8.085 \cdot 10^{-2}$ & $8.084 \cdot 10^{-2}$ & $8.083 \cdot 10^{-2}$ \\
$(0.5,0.5)$ & $7.684 \cdot 10^{-2}$ & $7.905 \cdot 10^{-2}$ & $7.896 \cdot 10^{-2}$ & $7.875 \cdot 10^{-2}$ & $7.872 \cdot 10^{-2}$ & $7.871 \cdot 10^{-2}$ \\
\hline
\end{tabular}

(b) Option 2

\begin{tabular}{c|c|c|c|c|c|c}
\hline$\mu$ & $\beta_{\mathrm{Br}}(\mu)$ & $\beta_{5}(\mu)$ & $\beta_{10}(\mu)$ & $\beta_{20}(\mu)$ & $\beta_{30}(\mu)$ & $\beta_{40}(\mu)$ \\
\hline$(0.1,0.1)$ & $1.073 \cdot 10^{-1}$ & $1.137 \cdot 10^{-1}$ & $1.136 \cdot 10^{-1}$ & $1.135 \cdot 10^{-1}$ & $1.134 \cdot 10^{-1}$ & $1.134 \cdot 10^{-1}$ \\
$(0.5,0.1)$ & $1.070 \cdot 10^{-1}$ & $1.134 \cdot 10^{-1}$ & $1.133 \cdot 10^{-1}$ & $1.132 \cdot 10^{-1}$ & $1.131 \cdot 10^{-1}$ & $1.131 \cdot 10^{-1}$ \\
$(0.3,0.3)$ & $9.583 \cdot 10^{-2}$ & $9.946 \cdot 10^{-2}$ & $9.951 \cdot 10^{-2}$ & $9.953 \cdot 10^{-2}$ & $9.955 \cdot 10^{-2}$ & $9.957 \cdot 10^{-2}$ \\
$(0.1,0.5)$ & $7.881 \cdot 10^{-2}$ & $8.034 \cdot 10^{-2}$ & $8.040 \cdot 10^{-2}$ & $8.049 \cdot 10^{-2}$ & $8.059 \cdot 10^{-2}$ & $8.072 \cdot 10^{-2}$ \\
$(0.5,0.5)$ & $7.684 \cdot 10^{-2}$ & $7.862 \cdot 10^{-2}$ & $7.862 \cdot 10^{-2}$ & $7.848 \cdot 10^{-2}$ & $7.858 \cdot 10^{-2}$ & $7.858 \cdot 10^{-2}$ \\
\hline
\end{tabular}

Table 3.1.: Values for the inf-sup constants $\beta_{N}(\mu)$ (see (2.17)) for several parameter values $\mu \in \mathcal{D}=[0.1,0.5]^{2}$ and $N$ evaluated using (a) Option 1 (see (2.77)) and (b) Option 2 (see (2.78)) based on a random sample $\mathcal{D}_{N}$ of parameter snapshots. 


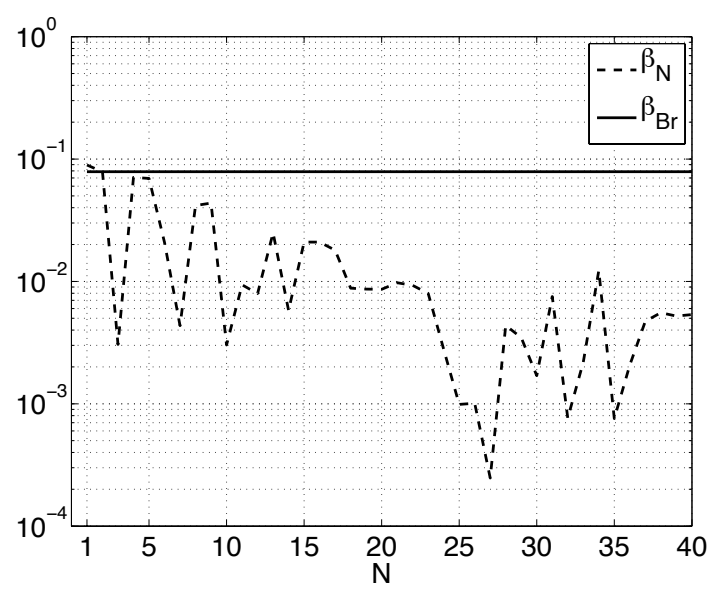

(a)

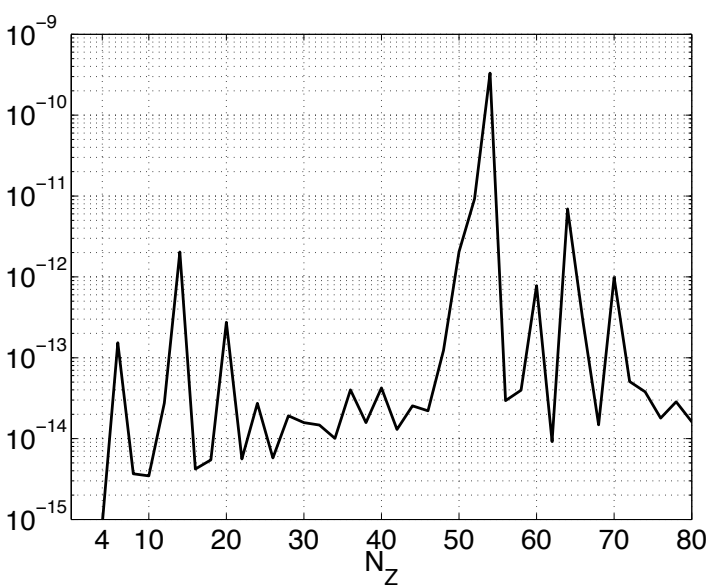

(b)

Figure 3.4.: Numerical results for $\mu=(0.1,0.5)$ using Option 0 (see $(2.70)$ ) based on a random sample $\mathcal{D}_{N}$ of parameter snapshots: (a) the inf-sup constant $\beta_{N}(\mu)$ (see (2.17)) shown as a function of $N$ together with the truth inf-sup constant $\beta_{\mathrm{Br}}(\mu)$ (see (2.11)); (b) the (absolute) distance $\left\|u_{N}(\mu)-B_{N}(\mu)^{-1} g_{N}(\mu)\right\|_{X}$ shown as a function of the total dimension $N_{Z}$.

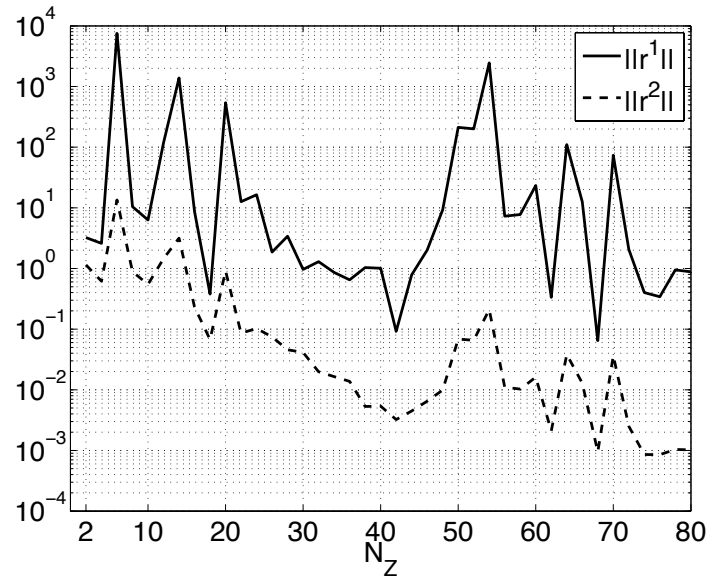

(a)

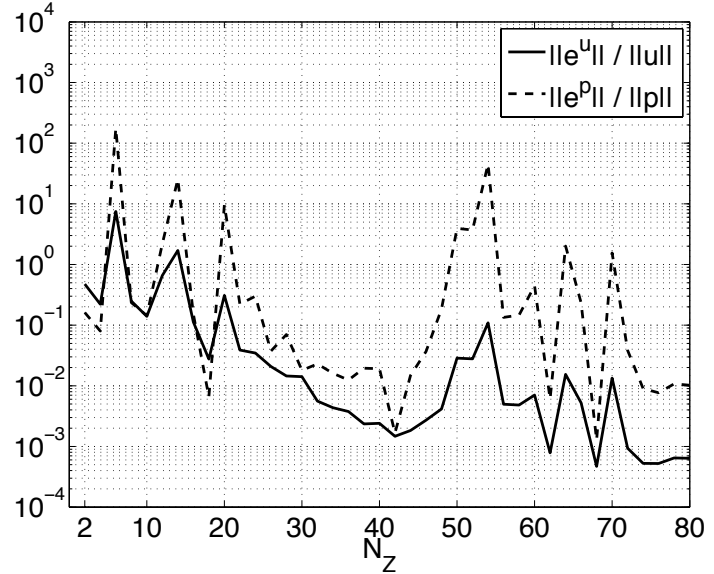

(b)

Figure 3.5.: Numerical results for $\mu=(0.1,0.5)$ using Option 0 (see (2.70)) based on a random sample $\mathcal{D}_{N}$ of parameter snapshots: (a) the residual dual norms $\left\|r_{N}^{1}(\cdot ; \mu)\right\|_{X^{\prime}},\left\|r_{N}^{2}(\cdot ; \mu)\right\|_{Y^{\prime}}$ (see (2.31)) and (b) the relative errors $\left\|e_{N}^{u}(\mu)\right\|_{X} /\|u(\mu)\|_{X},\left\|e_{N}^{p}(\mu)\right\|_{Y} /\|p(\mu)\|_{Y}$ (see (2.18)) shown as functions of the total dimension $N_{Z}$. 


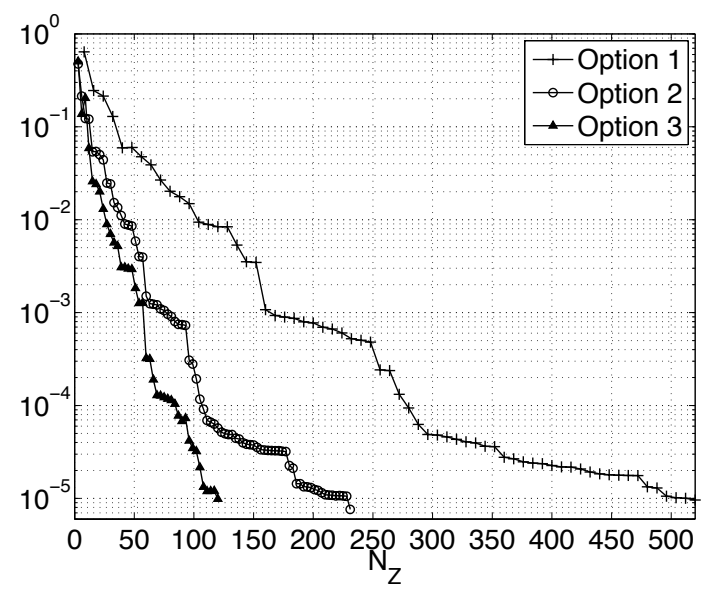

(a)

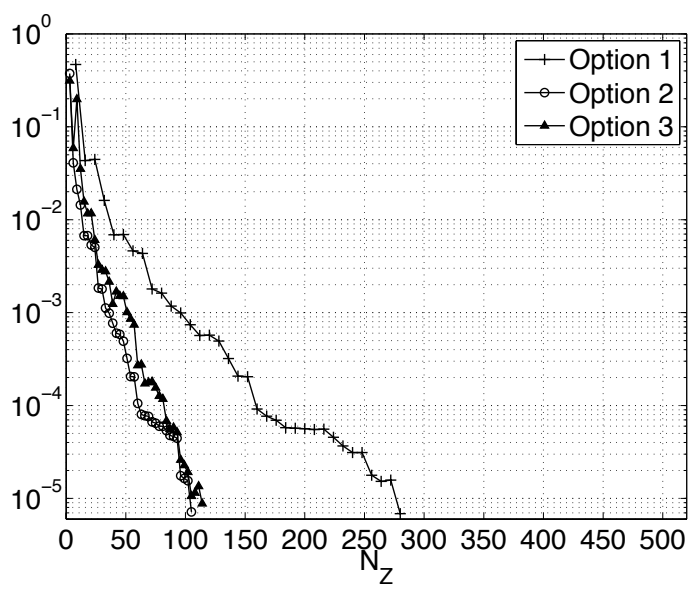

(b)

Figure 3.6.: Maximum relative errors (a) $\left\|e_{N}^{u}(\mu)\right\|_{X} /\|u(\mu)\|_{X}$ and (b) $\left\|e_{N}^{p}(\mu)\right\|_{Y} /\|p(\mu)\|_{Y}$ (see (2.18)) shown as functions of the total dimension $N_{Z}$ computed using Option 1, Option 2, and Option 3 (see Table 3.2) based on random samples $\mathcal{D}_{N}$ and $\mathcal{D}_{N}^{\prime}$ of parameter snapshots; the maximum is taken over 25 parameter values.

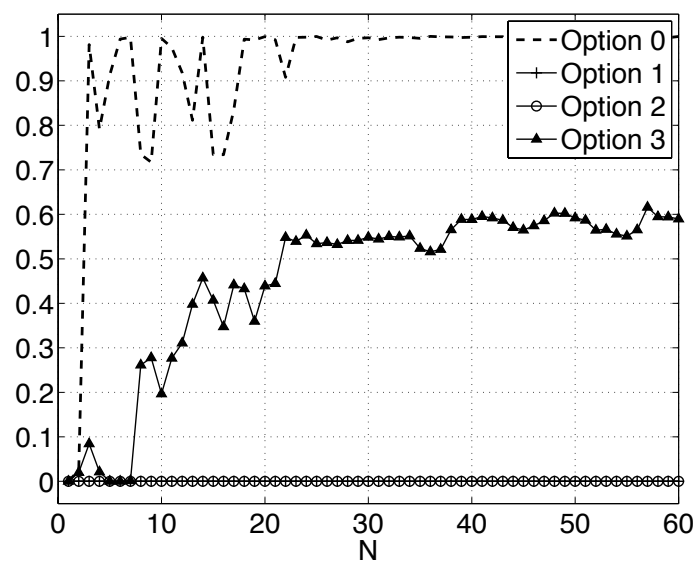

Figure 3.7.: Maximum relative distance $d_{N}^{\beta}(\mu)$ (see (2.101)) shown as a function of $N$ computed using Option 0, Option 1, Option 2, and Option 3 (see Table 3.2) based on random samples $\mathcal{D}_{N}$ and $\mathcal{D}_{N}^{\prime}$ of parameter snapshots; the maximum is taken over 25 parameter values. 
Figure 3.7 shows the relative distance $d_{N}^{\beta}(\mu)$ (see (2.101)) between $\beta_{N}(\mu)$ and the truth constant $\beta_{\mathrm{Br}}(\mu)$. We recall that a relative distance $d_{N}^{\beta}(\mu)<1$ implies that $\beta_{N}(\mu)$ is positive; $d_{N}^{\beta}(\mu)>0$ indicates that $\beta_{N}(\mu)<\beta_{\mathrm{Br}}(\mu)$. In case of Option 1 or Option 2 , we thus obtain $d_{N}^{\beta}(\mu)=0$ for all $\mu \in \mathcal{D}, N \in \mathbb{N}_{\max }$, due to (2.75). Option 3 (see (2.79)) provides in practice inf-sup constants $\beta_{N}(\mu)$ that are clearly positive but generally do not satisfy (2.75).

Figure 3.6 then verifies in practice what has been predicted by a priori and a posteriori error estimates (see §2.5): Small inf-sup constants $\beta_{N}(\mu)$ have more dramatic effects on $\left\|e_{N}^{p}(\mu)\right\|_{Y}$ than on $\left\|e_{N}^{u}(\mu)\right\|_{X}$. The error $\left\|e_{N}^{u}(\mu)\right\|_{X}$ profits from a large space $\operatorname{ker}\left(B_{N}(\mu)\right)$ but does not explicitly depend on $\beta_{N}(\mu)$. Therefore, it is not surprising that adding supremizers as in Options 1 and 2, providing through (2.75) comparatively large values for the inf-sup constants $\beta_{N}(\mu)$, particularly favors the approximations for the Lagrange multiplier. Here, the reduced basis approximations $p_{N}(\mu)$ for $p(\mu)$ converge much more rapidly than the approximations $u_{N}(\mu)$ for the primal variable $u(\mu)$ (see Fig. 3.6). Compared to Options 1 and 2, approximations for the primal variable improve considerably when constructing the reduced basis approximation spaces by using Option 3 (see Fig. 3.6(a)). Moreover, in (2.46) and (2.20), the smaller errors $\left\|e_{N}^{u}(\mu)\right\|_{X}$ seem to essentially compensate for the effects of the smaller inf-sup constants $\beta_{N}(\mu)$ : Approximations for the Lagrange multiplier are not significantly worse (see Fig. 3.6(b)).

Table 3.2 summarizes all options to construct the reduced basis approximation spaces together with their main respective properties.

\begin{tabular}{c|c|c}
\hline Option & $X_{N}$ & Special properties \\
\hline \hline 0 & $X_{N}^{0} \equiv \operatorname{span}\left\{u\left(\mu_{n}\right) \mid 1 \leq n \leq N\right\}(2.70)$ & $\begin{array}{c}\beta_{N}(\mu)>0 \text { with } d_{N}^{\beta}(\mu) \text { large } \\
\operatorname{dim}\left(X_{N}\right)=\operatorname{dim}\left(Y_{N}\right)\end{array}$ \\
\hline 1 & $X_{N}^{1} \equiv X_{N}^{0} \oplus \operatorname{span}\left\{T^{k} \xi_{n} \mid 1 \leq n \leq N, 1 \leq k \leq Q_{b}\right\}(2.77)$ & $\begin{array}{c}\beta_{N}(\mu) \geq \beta_{\mathrm{Br}}(\mu) \\
\text { (in theory and practice) }\end{array}$ \\
\hline 2 & $X_{N}^{2} \equiv X_{N}^{0} \oplus \operatorname{span}\left\{T_{\mu_{n}} \xi_{n} \mid 1 \leq n \leq N\right\}(2.78)$ & $\begin{array}{c}\beta_{N}(\mu) \geq \beta_{\mathrm{Br}}(\mu) \text { (in practice) } \\
\operatorname{dim}\left(X_{N}^{2}\right) \ll \operatorname{dim}\left(X_{N}^{1}\right)\end{array}$ \\
\hline 3 & $X_{N}^{3} \equiv X_{N}^{0} \oplus \operatorname{span}\left\{u\left(\mu_{n}^{\prime}\right) \mid 1 \leq n \leq N\right\}(2.79)$ & $\begin{array}{c}\beta_{N}(\mu)>0 \text { with } d_{N}^{\beta}(\mu) \text { small } \\
\text { good space } \operatorname{ker}\left(B_{N}(\mu)\right)\end{array}$ \\
\hline
\end{tabular}

Table 3.2.: Options to construct the reduced basis approximation space $X_{N}$ for the primal variable, where the reduced basis approximation space $Y_{N}$ for the Lagrange multiplier is given as in (2.69).

\subsubsection{Adaptive Sampling Procedures}

We now discuss numerical results for the adaptive sampling procedures developed in $§ 2.7$.

First, we note that our Stokes model problem is clearly symmetric and therefore, $\Delta_{N}^{u \text {,sym }}(\mu)$, $\Delta_{N}^{p \text {,sym }}(\mu)$, and $\tilde{\Delta}_{N}^{u, \text { sym }}(\mu)$ represent valid upper bounds for the errors in our reduced basis approximations (see $\S 2.4$ ). All sampling procedures presented in this chapter are now based on an exhaustive random sample $\Sigma \subset \mathcal{D}$ of size $|\Sigma|=4,900$ and the relative error bound $\Delta_{N}(\mu) \equiv \tilde{\Delta}_{N}^{u, \text { sym }}(\mu) /\left\|u_{N}(\mu)\right\|_{X}($ see $(2.67))$.

Figure 3.8 shows results for Algorithm 2 using different values for $\delta_{\text {tol }}^{\beta} \in(0,1)$; a direct 


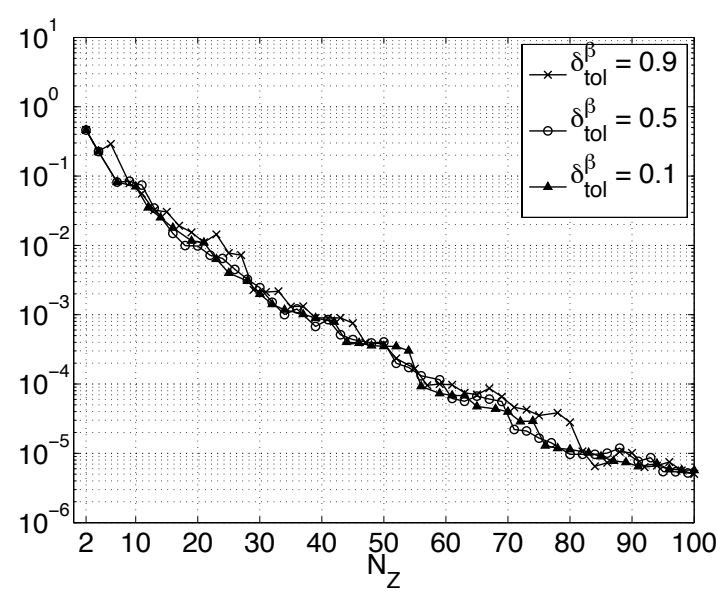

(a)

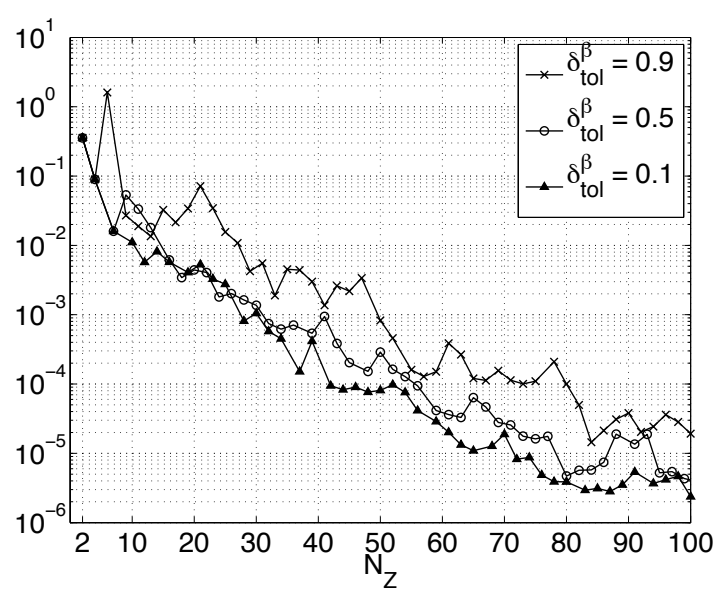

(b)

Figure 3.8.: Numerical results for Algorithm 2 (see $\S 2.7$ ) using $\delta_{\text {tol }}^{\beta} \in\{0.9,0.5,0.1\}$ : Maximum relative errors (a) $\left\|e_{N}^{u}(\mu)\right\|_{X} /\|u(\mu)\|_{X}$ and (b) $\left\|e_{N}^{p}(\mu)\right\|_{Y} /\|p(\mu)\|_{Y}$ (see (2.18)) are shown as functions of $N_{Z}$; the maximum is taken over 25 parameter values.

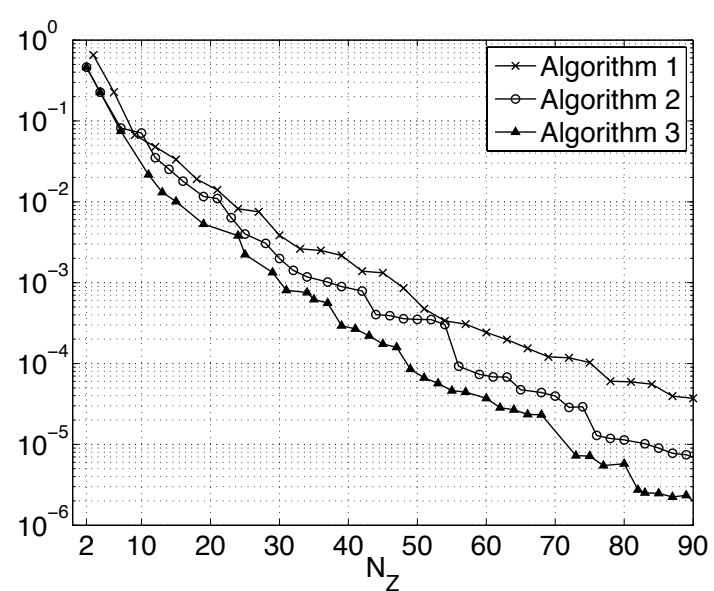

(a)

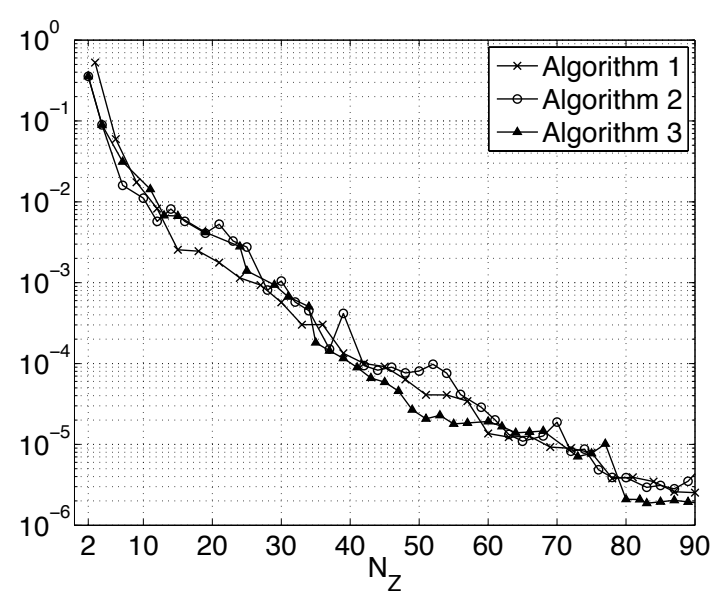

(b)

Figure 3.9.: Comparison of Algorithm 2 and Algorithm 3 (both using $\delta_{\text {tol }}^{\beta}=0.1$ ) to the standard greedy procedure in Algorithm 1 (see §2.7): Maximum relative errors (a) $\left\|e_{N}^{u}(\mu)\right\|_{X} /\|u(\mu)\|_{X}$ and (b) $\left\|e_{N}^{p}(\mu)\right\|_{Y} /\|p(\mu)\|_{Y}$ (see (2.18)) are shown as functions of $N_{Z}$; the maximum is taken over 25 parameter values. 


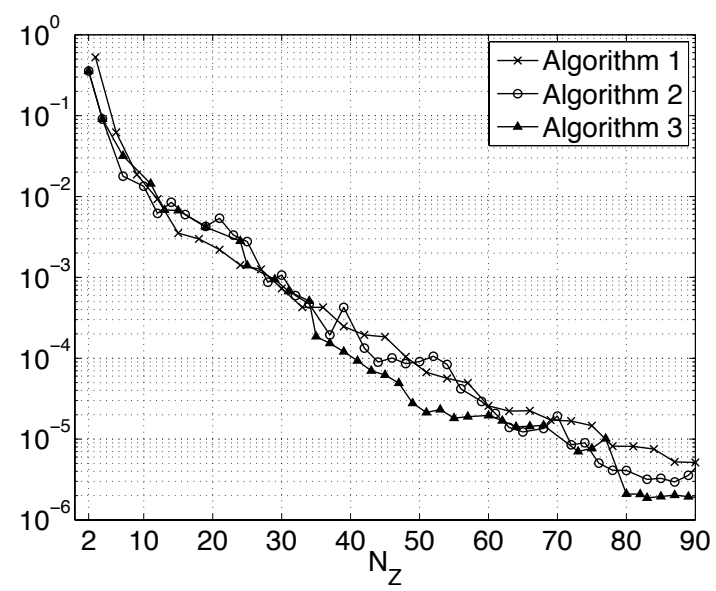

Figure 3.10.: Comparison of Algorithm 2 and Algorithm 3 (both using $\delta_{\text {tol }}^{\beta}=0.1$ ) to the standard greedy procedure in Algorithm 1 (see §2.7): Maximum relative errors $\left\|e_{N}(\mu)\right\|_{Z} /\|(u(\mu), p(\mu))\|_{Z}$ (see (2.18)) are shown as functions of $N_{Z}$; the maximum is taken over 25 parameter values.

comparison with Algorithm 3 is then given in Fig. 3.9 and Fig. 3.10. Again, it is verified in practice what a priori and a posteriori error bounds in $\S 2.3 .3$ and Remark 2.4.4 indicate: Small inf-sup constants $\beta_{N}(\mu)$ have much stronger effects on $\left\|e_{N}^{p}(\mu)\right\|_{Y}$ than on $\left\|e_{N}^{u}(\mu)\right\|_{X}$. Even for fairly large tolerances $\delta_{\text {tol }}^{\beta}$, we obtain approximation spaces $X_{N}, Y_{N}$ providing very accurate approximations $u_{N}(\mu)$ that do not necessarily further improve for lower values of $\delta_{\text {tol }}^{\beta}$ (see Fig. 3.8(a)). In contrast, accurate approximations for the Lagrange multiplier require a smaller tolerance $\delta_{\text {tol }}^{\beta}$ and improve considerably in the dependence of decreasing values of $\delta_{\text {tol }}^{\beta}$ (see Fig. 3.8(b)). The errors $\left\|e_{N}^{u}(\mu)\right\|_{X}$ benefit primarily from a good testing space $\operatorname{ker}(B(\mu)) \cap X_{N} \subseteq \operatorname{ker}\left(B_{N}(\mu)\right)$ (see $\left.\S 2.5\right)$ as achieved in Algorithm 3 (see Fig. 3.9(a)).

Figure 3.9 and Figure 3.10 moreover demonstrate that the standard greedy procedure (see Algorithm 1) may indeed generally be inefficient: For our model problem, Algorithm 2 provides accurate approximations for the primal variable at much less computational cost; using Algorithm 3, we obtain even greater computational savings compared to Algorithm 1 of up to $35 \%$.

\subsubsection{A Posteriori Error Bounds}

We have thus seen how different constructions of the reduced basis approximation spaces affect the convergence of the reduced basis approximations. However, as the associated truth solutions are usually not computed, the errors in our reduced basis approximations are unknown quantities that may only be estimated by a posteriori error bounds. We now comment on the sharpness and computational efficiency of the rigorous a posteriori error bounds developed in $\S 2.4$.

We first compare the proposed a posteriori error bounds with respect to sharpness. Figure 3.11 and Figure 3.12 show the maximum errors in the reduced basis approximations for the primal variable and the Lagrange multiplier, respectively, together with the respective 


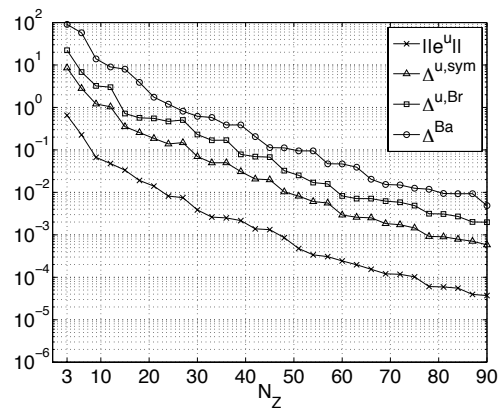

(a)

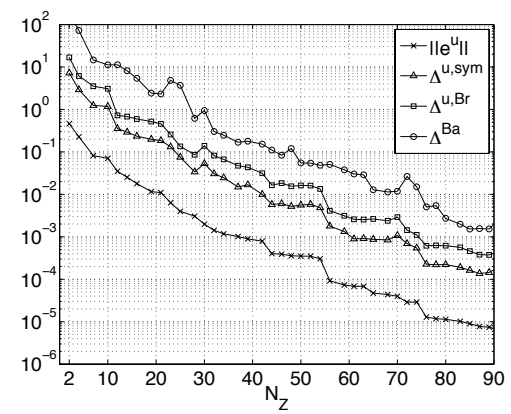

(b)

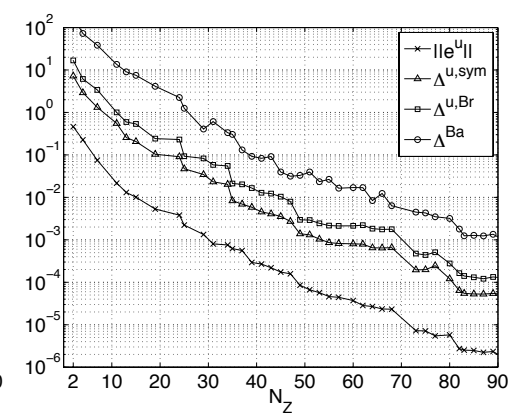

(c)

Figure 3.11.: Maximum relative error $\left\|e_{N}^{u}(\mu)\right\|_{X} /\|u(\mu)\|_{X}$ and maximum relative error bounds $\Delta_{N}^{u, \text { sym }}(\mu) /\|u(\mu)\|_{X}, \quad \Delta_{N}^{u, \operatorname{Br}}(\mu) /\|u(\mu)\|_{X}$, and $\Delta_{N}^{\mathrm{Ba}}(\mu) /\|u(\mu)\|_{X} \quad$ (see (2.18), (2.55), (2.37), and (2.35)) shown as functions of $N_{Z}$ for (a) Algorithm 1, (b) Algorithm 2 with $\delta_{\text {tol }}^{\beta}=0.1$, and (c) Algorithm 3 with $\delta_{\text {tol }}^{\beta}=0.1$ (see $\S 2.7$ ); the maximum is taken over 25 parameter values; the computation of the error bounds is based on the exact constants $\alpha_{a}(\mu), \gamma_{a}(\mu), \beta_{\mathrm{Br}}(\mu)$, and $\beta_{\mathrm{Ba}}(\mu)$.

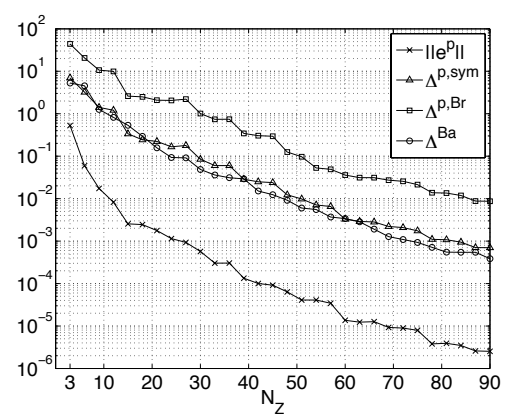

(a)

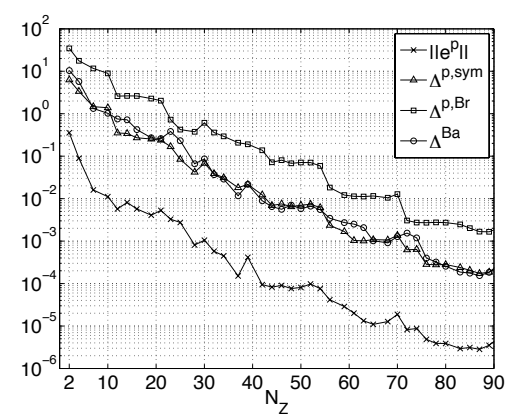

(b)

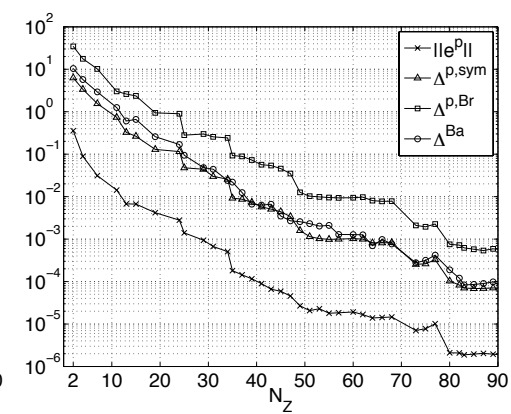

(c)

Figure 3.12.: Maximum relative error $\left\|e_{N}^{p}(\mu)\right\|_{Y} /\|p(\mu)\|_{Y}$ and maximum relative error bounds $\Delta_{N}^{p, \text { sym }}(\mu) /\|p(\mu)\|_{Y}, \quad \Delta_{N}^{p, \operatorname{Br}}(\mu) /\|p(\mu)\|_{Y}$, and $\Delta_{N}^{\mathrm{Ba}}(\mu) /\|p(\mu)\|_{Y} \quad$ (see (2.18), (2.56), (2.38), and (2.35)) shown as functions of $N_{Z}$ for (a) Algorithm 1, (b) Algorithm 2 with $\delta_{\text {tol }}^{\beta}=0.1$, and (c) Algorithm 3 with $\delta_{\text {tol }}^{\beta}=0.1$ (see $\S 2.7$ ); the maximum is taken over 25 parameter values; the computation of the error bounds is based on the exact constants $\alpha_{a}(\mu), \gamma_{a}(\mu), \beta_{\mathrm{Br}}(\mu)$, and $\beta_{\mathrm{Ba}}(\mu)$. 
(a) Algorithm 1

\begin{tabular}{c|c|c|c|c|c|c|c|c|c|c|c}
\hline$N$ & $N_{Z}$ & $\eta_{N}^{u, \text { sym }}$ & $\eta_{N}^{u, \text { sym }}$ & $\eta_{N}^{u, \mathrm{Br}}$ & $\eta_{N}^{u, \mathrm{Ba}}$ & $\eta_{N}^{p, \text { sym }}$ & $\eta_{N}^{p, \mathrm{Br}}$ & $\eta_{N}^{p, \mathrm{Ba}}$ & $\eta_{N}^{\mathrm{sym}}$ & $\eta_{N}^{\mathrm{Br}}$ & $\eta_{N}^{\mathrm{Ba}}$ \\
\hline 5 & 15 & 13.21 & 20.68 & 58.04 & 353.6 & 202.1 & 1534 & 296.4 & 148.0 & 1238 & 203.6 \\
10 & 30 & 12.34 & 19.94 & 63.93 & 277.5 & 292.5 & 2268 & 326.5 & 168.6 & 1559 & 186.7 \\
15 & 45 & 13.22 & 19.57 & 57.42 & 309.9 & 331.5 & 3223 & 417.2 & 201.2 & 1598 & 228.6 \\
20 & 60 & 13.29 & 19.35 & 58.40 & 255.0 & 355.9 & 3240 & 415.7 & 208.1 & 1580 & 207.1 \\
25 & 75 & 11.97 & 18.77 & 57.92 & 242.9 & 405.8 & 3513 & 476.3 & 198.4 & 1560 & 216.4 \\
30 & 90 & 13.55 & 19.93 & 54.23 & 282.3 & 399.2 & 3423 & 453.6 & 227.0 & 1678 & 232.6 \\
35 & 105 & 13.62 & 20.44 & 53.93 & 281.7 & 432.1 & 2502 & 519.8 & 235.2 & 1535 & 234.9 \\
40 & 120 & 14.28 & 21.53 & 57.49 & 295.4 & 482.6 & 3317 & 495.2 & 253.0 & 1526 & 253.1 \\
\hline
\end{tabular}

(b) Algorithm 2

\begin{tabular}{c|c|c|c|c|c|c|c|c|c|c|c}
\hline$N$ & $N_{Z}$ & $\tilde{\eta}_{N}^{u, \text { sym }}$ & $\eta_{N}^{u, \text { sym }}$ & $\eta_{N}^{u, \mathrm{Br}}$ & $\eta_{N}^{u, \mathrm{Ba}}$ & $\eta_{N}^{p, \text { sym }}$ & $\eta_{N}^{p, \mathrm{Br}}$ & $\eta_{N}^{p, \mathrm{Ba}}$ & $\eta_{N}^{\text {sym }}$ & $\eta_{N}^{\mathrm{Br}}$ & $\eta_{N}^{\mathrm{Ba}}$ \\
\hline 5 & 12 & 16.11 & 23.89 & 55.18 & 715.1 & 98.47 & 756.3 & 142.5 & 88.04 & 673.4 & 139.7 \\
10 & 23 & 22.45 & 32.53 & 62.65 & 1894 & 121.8 & 799.9 & 234.8 & 99.72 & 700.7 & 233.0 \\
15 & 34 & 13.57 & 25.31 & 68.15 & 425.1 & 152.8 & 766.6 & 253.9 & 130.7 & 660.6 & 216.6 \\
20 & 46 & 13.17 & 20.31 & 61.41 & 505.3 & 224.0 & 1268 & 307.0 & 172.7 & 1081 & 236.1 \\
25 & 56 & 16.44 & 27.51 & 63.04 & 1013 & 97.71 & 550.1 & 258.9 & 94.87 & 523.1 & 250.8 \\
30 & 68 & 14.63 & 23.48 & 61.14 & 583.5 & 237.9 & 1287 & 294.2 & 189.6 & 1023 & 233.8 \\
35 & 78 & 14.80 & 22.35 & 59.63 & 707.3 & 126.3 & 843.6 & 269.0 & 118.3 & 778.2 & 251.5 \\
40 & 89 & 14.13 & 23.70 & 61.52 & 613.1 & 162.4 & 800.6 & 285.4 & 139.3 & 685.6 & 244.3 \\
\hline
\end{tabular}

(c) Algorithm 3

\begin{tabular}{c|c|c|c|c|c|c|c|c|c|c|c}
\hline$N$ & $N_{Z}$ & $\eta_{N}^{u, \text { sym }}$ & $\eta_{N}^{u, \text { sym }}$ & $\eta_{N}^{u, \text { Br }}$ & $\eta_{N}^{u, \mathrm{Ba}}$ & $\eta_{N}^{p, \text { sym }}$ & $\eta_{N}^{p, \mathrm{Br}}$ & $\eta_{N}^{p, \mathrm{Ba}}$ & $\eta_{N}^{\text {sym }}$ & $\eta_{N}^{\mathrm{Br}}$ & $\eta_{N}^{\mathrm{Ba}}$ \\
\hline 5 & 13 & 18.36 & 32.56 & 68.23 & 1146 & 85.38 & 793.3 & 162.2 & 79.09 & 755.4 & 157.9 \\
10 & 29 & 22.08 & 33.22 & 72.73 & 717.1 & 183.0 & 996.8 & 219.0 & 152.9 & 830.9 & 182.5 \\
15 & 39 & 15.56 & 26.58 & 63.64 & 446.0 & 137.4 & 721.9 & 195.0 & 123.8 & 649.1 & 173.1 \\
20 & 49 & 15.13 & 24.88 & 69.96 & 699.3 & 118.1 & 807.8 & 174.9 & 104.2 & 753.7 & 162.0 \\
25 & 60 & 19.45 & 33.86 & 71.13 & 1034 & 130.3 & 577.4 & 206.5 & 115.1 & 509.1 & 195.4 \\
30 & 73 & 20.00 & 40.18 & 97.51 & 976.6 & 102.5 & 582.7 & 187.7 & 94.86 & 538.1 & 179.6 \\
35 & 83 & 16.90 & 28.72 & 71.93 & 821.5 & 136.3 & 692.0 & 217.4 & 124.6 & 631.3 & 198.3 \\
40 & 93 & 17.87 & 33.31 & 82.45 & 973.4 & 196.7 & 1012 & 301.9 & 165.4 & 849.5 & 288.4 \\
\hline
\end{tabular}

Table 3.3.: Maximum effectivities $\tilde{\eta}_{N}^{u, \text { sym }}(\mu)=\tilde{\Delta}_{N}^{u, \text { sym }}(\mu) /\left\|e_{N}^{u}(\mu)\right\|_{X, \mu} \quad($ see $\quad(2.68)$ ), $\eta_{N}^{u, \text { sym }}(\mu)=\Delta_{N}^{u, \text { sym }}(\mu) /\left\|e_{N}^{u}(\mu)\right\|_{X}, \eta_{N}^{p, \text { sym }}(\mu) \equiv \Delta_{N}^{p, \text { sym }}(\mu) /\left\|e_{N}^{p}(\mu)\right\|_{Y}, \eta_{N}^{\text {sym }}(\mu)=$ $\Delta_{N}^{\mathrm{sym}}(\mu) /\left\|e_{N}(\mu)\right\|_{Z}$ (see $(2.57)$ and $\left.(2.66)\right)$, and $\eta_{N}^{u, \operatorname{Br}}(\mu) \equiv \Delta_{N}^{u, \operatorname{Br}}(\mu) /\left\|e_{N}^{u}(\mu)\right\|_{X}$, $\eta_{N}^{p, \operatorname{Br}}(\mu) \equiv \Delta_{N}^{p, \operatorname{Br}}(\mu) /\left\|e_{N}^{p}(\mu)\right\|_{Y}, \quad \eta_{N}^{\mathrm{Br}}(\mu)=\Delta_{N}^{\mathrm{Br}}(\mu) /\left\|e_{N}(\mu)\right\|_{Z}, \quad \eta_{N}^{u, \mathrm{Ba}}(\mu) \equiv$ $\Delta_{N}^{\mathrm{Ba}}(\mu) /\left\|e_{N}^{u}(\mu)\right\|_{X}, \eta_{N}^{p, \mathrm{Ba}}(\mu) \equiv \Delta_{N}^{\mathrm{Ba}}(\mu) /\left\|e_{N}^{p}(\mu)\right\|_{Y}, \eta_{N}^{\mathrm{Ba}}(\mu)=\Delta_{N}^{\mathrm{Ba}}(\mu) /\left\|e_{N}(\mu)\right\|_{Z}$ (see (2.39), (2.36), and (2.48)) for (a) Algorithm 1, (b) Algorithm 2 with $\delta_{\text {tol }}^{\beta}=0.1$, and (c) Algorithm 3 with $\delta_{\text {tol }}^{\beta}=0.1$ (see $\S 2.7$ ); the maximum is taken over 25 parameter values; the computation of the error bounds is based on the exact constants $\alpha_{a}(\mu), \gamma_{a}(\mu), \beta_{\mathrm{Br}}(\mu)$, and $\beta_{\mathrm{Ba}}(\mu)$. 


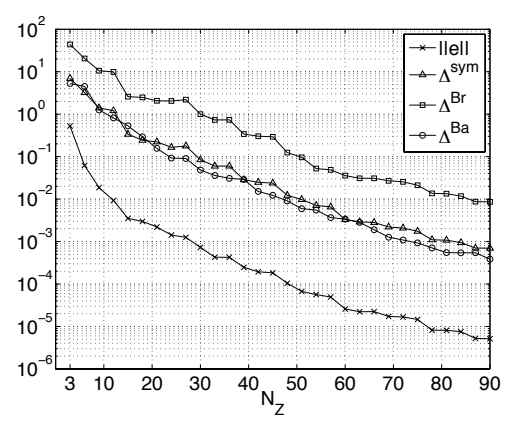

(a)

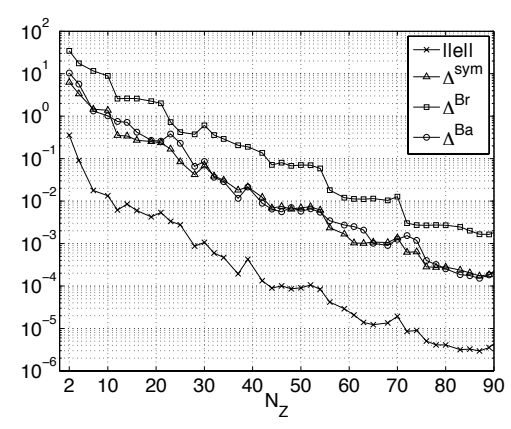

(b)

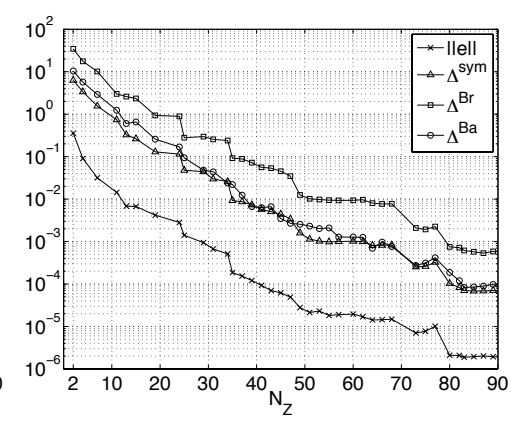

(c)

Figure 3.13.: Maximum relative error $\left\|e_{N}(\mu)\right\|_{Z} /\|(u(\mu), p(\mu))\|_{Z}$ and maximum relative error bounds $\Delta_{N}^{\mathrm{sym}}(\mu) /\|(u(\mu), p(\mu))\|_{Z}, \quad \Delta_{N}^{\mathrm{Br}}(\mu) /\|(u(\mu), p(\mu))\|_{Z}$, and $\Delta_{N}^{\mathrm{Ba}}(\mu) /\|(u(\mu), p(\mu))\|_{Z}$ (see (2.18), (2.65), (2.47), and (2.35)) shown as functions of $N_{Z}$ for (a) Algorithm 1, (b) Algorithm 2 with $\delta_{\text {tol }}^{\beta}=0.1$, and (c) Algorithm 3 with $\delta_{\text {tol }}^{\beta}=0.1$ (see $\S 2.7$ ); the maximum is taken over 25 parameter values; the computation of the error bounds is based on the exact constants $\alpha_{a}(\mu), \gamma_{a}(\mu), \beta_{\mathrm{Br}}(\mu)$, and $\beta_{\mathrm{Ba}}(\mu)$.

error bounds $\Delta_{N}^{u, \text { sym }}(\mu), \Delta_{N}^{u, \mathrm{Br}}(\mu), \Delta_{N}^{p, \text { sym }}(\mu), \Delta_{N}^{p, \mathrm{Br}}(\mu)$, and $\Delta_{N}^{\mathrm{Ba}}(\mu)$ (see $\S 2.4$ ); Figure 3.13 shows the maximum total error in the reduced basis approximation and associated error bounds $\Delta_{N}^{\mathrm{sym}}(\mu), \Delta_{N}^{\mathrm{Br}}(\mu)$, and $\Delta_{N}^{\mathrm{Ba}}(\mu)$ (see $\left.\S 2.4\right)$. The maximum is computed over a sample of 25 parameter values. For this sample, to analyze only the effects of the a posteriori error bound formulations and eliminate contributions of the SCM, the exact constants $\alpha_{a}(\mu)$, $\gamma_{a}(\mu), \beta_{\mathrm{Br}}(\mu)$, and $\beta_{\mathrm{Ba}}(\mu)$ rather than the lower/upper bounds (2.29), (2.30) are used. Effectivities associated with the error bounds are given in Table 3.3. With maximum effectivities essentially ranging from 50 to $80, \Delta_{N}^{u, \mathrm{Br}}(\mu)$ represents a reasonably sharp bound for the error in the reduced basis approximation $u_{N}(\mu)$ (see Fig. 3.11 and Table 3.3). However, exploiting the symmetry of the problem, $\Delta_{N}^{u, \text { sym }}(\mu)$ provides a sharper bound not only in theory (see §2.4) but also in practice: Here, effectivities essentially vary between 20 and 40. Furthermore, it is not surprising to observe that the best results are achieved by the bound $\tilde{\Delta}_{N}^{u, \operatorname{sym}}(\mu)$ that overestimates the error in the reduced basis approximations for the primal variable measured in the energy norm (see (2.68)) only by a factor of approximately 15 (see Table 3.3). In contrast, $\Delta_{N}^{p, \mathrm{Br}}(\mu)$ and $\Delta_{N}^{p \text {,sym }}(\mu)$ estimate the error in $p_{N}(\mu)$ rather pessimistically (see Fig. 3.12). For $\Delta_{N}^{p, \operatorname{Br}}(\mu)$, exact effectivities strongly depend on how the underlying reduced basis approximation spaces are constructed: Using Algorithm 1, we obtain effectivity values of order $3 \cdot 10^{3}$ (see Table $3.3(\mathrm{a})$ ); in this case, $\Delta_{N}^{\mathrm{Ba}}(\mu)$ clearly represents a much sharper bound. Yet, using Algorithm 2 or Algorithm 3, the difference is not as large (see Table 3.3(b) and (c)). For $\Delta_{N}^{p \text {,sym }}(\mu)$, we still obtain rather large effectivities with average values 362, 161, and 127 in case of Algorithm 1, Algorithm 2, and Algorithm 3, respectively. However, in neither case, do $\Delta_{N}^{u \text {,sym }}(\mu)$ and $\Delta_{N}^{p \text {,sym }}(\mu)$ perform worse than $\Delta_{N}^{\mathrm{Ba}}(\mu)$ but generally provide bounds for the errors in the primal and Lagrange multiplier variables that are much more accurate (see Table 3.3).

We now turn to the computational efficiency of the a posteriori error bounds. The SCM 

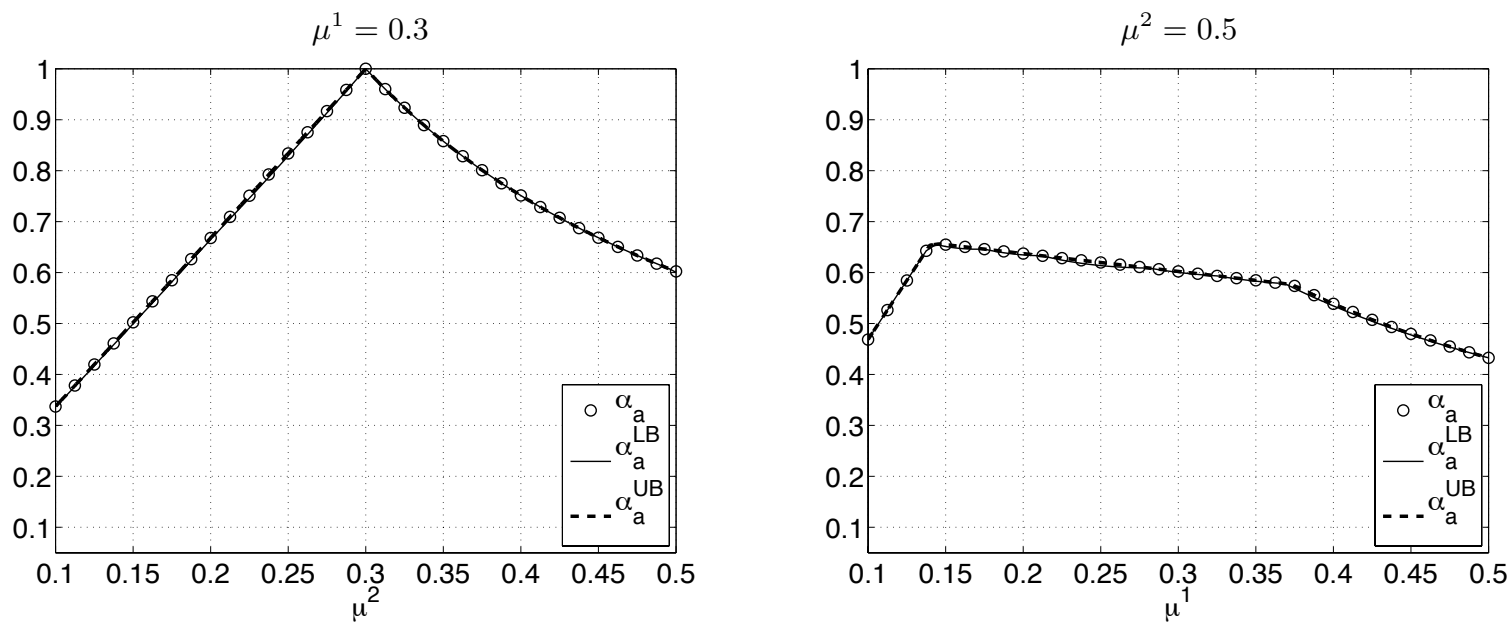

Figure 3.14.: SCM lower and upper bounds $\alpha_{a}^{\mathrm{LB}}(\mu)$ and $\alpha_{a}^{\mathrm{UB}}(\mu)$ for the coercivity constants $\alpha_{a}(\mu)($ see $(2.10),(2.29))$ where $|\Xi|=4,225, M_{\alpha}=\infty, \delta_{\text {tol }}^{\mathrm{SCM}}=0.01$, and $K_{\max }=35$.
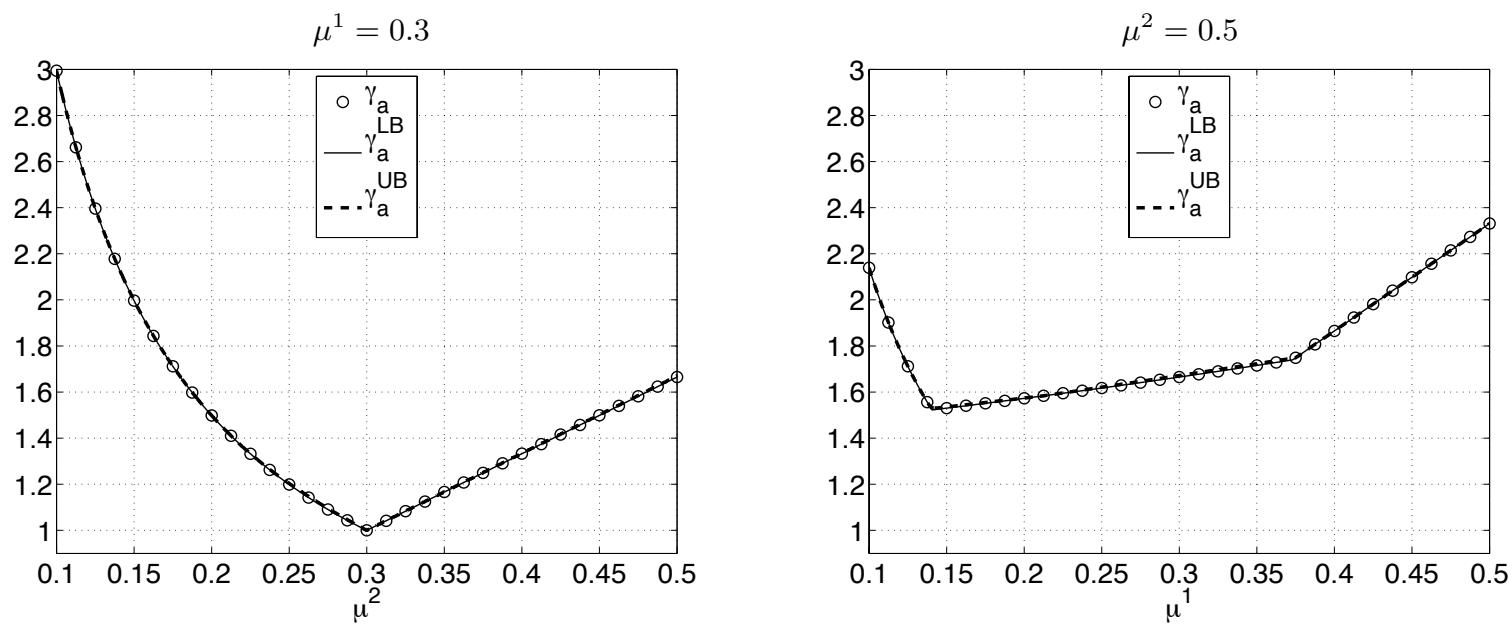

Figure 3.15.: SCM lower and upper bounds $\gamma_{a}^{\mathrm{LB}}(\mu)$ and $\gamma_{a}^{\mathrm{UB}}(\mu)$ for the continuity constants $\gamma_{a}(\mu)$ (see $(2.8),(2.29)$ ) where $|\Xi|=4,225, M_{\alpha}=\infty, \delta_{\text {tol }}^{\mathrm{SCM}}=0.01$, and $K_{\max }=12$. 

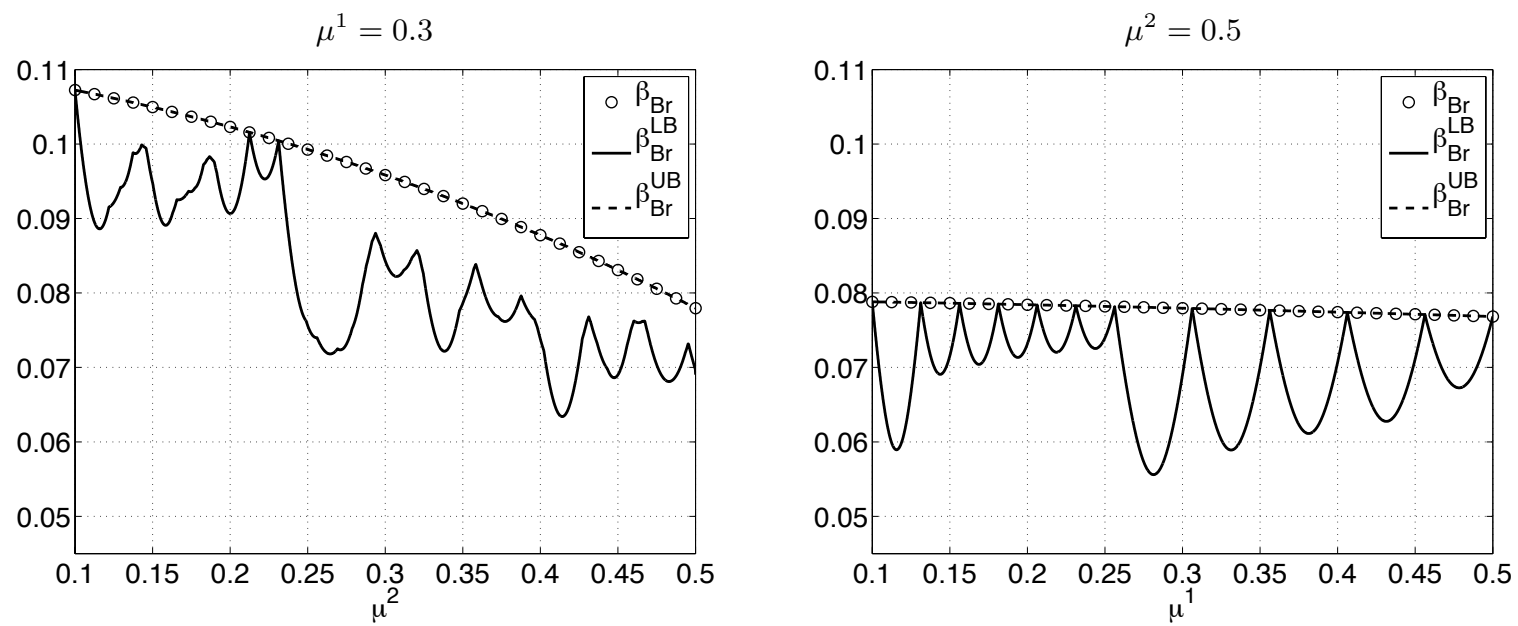

Figure 3.16.: SCM lower and upper bounds $\beta_{\mathrm{Br}}^{\mathrm{LB}}(\mu)$ and $\beta_{\mathrm{Br}}^{\mathrm{UB}}(\mu)$ for the Brezzi inf-sup constants $\beta_{\mathrm{Br}}(\mu)$ (see $(2.11),(2.30)$ ) where $|\Xi|=4,225, M_{\alpha}=\infty, \delta_{\text {tol }}^{\mathrm{SCM}}=0.05$, and $K_{\max }=163$.

(see §2.6.2) enables the (Online-)efficient estimation of the constants $\alpha_{a}(\mu), \gamma_{a}(\mu)$, and $\beta_{\mathrm{Br}}(\mu)$. We here apply the method for $M_{\alpha}=\infty$ and an exhaustive sample $\Xi \subset \mathcal{D}$ of size $|\Xi|=4,225$. We set the SCM tolerance $\delta_{\text {tol }}^{\mathrm{SCM}}=0.01$ (resp., 0.5 ) and obtain $K_{\max }=35$ (resp., 6) for the coercivity constants $\alpha_{a}(\mu)$ and $K_{\max }=12$ (resp., 5) for the continuity constants $\gamma_{a}(\mu)$. For the Brezzi inf-sup constants $\beta_{\mathrm{Br}}(\mu)$, we set $\delta_{\text {tol }}^{\mathrm{SCM}}=0.5$ (resp., 0.75) and obtain $K_{\max }=163$ (resp., 111). We receive very accurate (Online-)efficient bounds $\alpha_{a}^{\mathrm{LB}}(\mu)$, $\gamma_{a}^{\mathrm{UB}}(\mu)$, and $\beta_{\mathrm{Br}}^{\mathrm{LB}}(\mu)$ (see Fig. 3.14, Fig. 3.15, and Fig. 3.16), providing a posteriori error bounds $\Delta_{N}^{u, \operatorname{Br}}(\mu), \Delta_{N}^{u, \text { sym }}(\mu), \tilde{\Delta}_{N}^{u, \text { sym }}(\mu)$ and $\Delta_{N}^{p, \operatorname{Br}}(\mu), \Delta_{N}^{p, \text { sym }}(\mu)$ that essentially coincide with their values based on the evaluation of the exact constants; associated effectivities thus remain the same as shown in Table 3.3.

Using a $2.66 \mathrm{GHz}$ Intel Core 2 Duo processor, Offline computations necessary for the SCM applied to the Babuška inf-sup constants $\beta_{\mathrm{Ba}}(\mu)$ are infeasible. The difficulty here lies in the computations required for the SCM bounding box $\mathcal{B}$ (see (2.95)): The differential operator associated with the Babuška inf-sup constants exhibits a $\mu$-affine decomposition (2.7) that involves $Q$ terms where $\max \left\{Q_{a}, Q_{b}\right\} \leq Q \leq Q_{a}+Q_{b}$; the SCM bounding box then requires the solution of $Q(1+Q)$ generalized eigenvalue problems. (Recall that in our model problem, we have $Q_{a}=10$ and $Q_{b}=6$; here, $Q=15$.) However, even the solution of a single generalized eigenvalue problem associated with $\mathcal{B}$ is a computational challenge: System matrices on the left-hand side are dense, symmetric, not necessarily positive definite, and of a very low rank due to the geometric transformations involved. Consequently, even for our model problem where geometric variations are still relatively simple, we would have to resort to one of the other approaches discussed in Remark 2.6.1 to obtain (Online-)efficient lower bounds to the inf-sup constants $\beta_{\mathrm{Ba}}(\mu)$ (see (2.30)). No matter which of the currently existing methods is used, though, the (Online-)efficient evaluation of $\Delta_{N}^{\mathrm{Ba}}(\mu)$ requires either Offline costs that are prohibitively expensive compared to the ones needed for $\Delta_{N}^{u, \operatorname{Br}}(\mu)$, $\Delta_{N}^{u, \text { sym }}(\mu), \tilde{\Delta}_{N}^{u, \text { sym }}(\mu)$ and $\Delta_{N}^{p, \operatorname{Br}}(\mu), \Delta_{N}^{p, \text { sym }}(\mu)$, or a significant loss in terms of accuracy 
causing associated effectivities to be much worse than in Table 3.3.

Remark 3.3.1. In case of our model problem, the error bounds introduced in Remark 2.4.5 in fact essentially coincide with $\Delta_{N}^{u, \mathrm{Br}}(\mu)$ and $\Delta_{N}^{p, \mathrm{Br}}(\mu)$, respectively, and we therefore do not discuss them separately at this point.

\subsubsection{Computation Times}

We may now discuss the Online computation times for the proposed methods. For comparison, once the $\mu$-independent parts in (2.7) have been formed, direct computation of the truth approximation $(u(\mu), p(\mu))$ (i.e., assembly and solution of $(2.13))$ takes on average 6.5 seconds. The rigorous and efficient error bounds $\Delta_{N}^{u, \text { sym }}(\mu)$ and $\Delta_{N}^{p, \text { sym }}(\mu)$ allow us to choose the reduced basis system dimension $N_{Z}$ just large enough to obtain a desired accuracy. In case of Algorithm 1, we need $N_{Z}=51$ to achieve a prescribed accuracy of roughly $1 \%$ or better in the reduced basis approximations $u_{N}(\mu)$ (see Fig. 3.11(a)). Once the database has been loaded, the Online calculation of $\left(u_{N}(\mu), p_{N}(\mu)\right)$ (i.e., assembly and solution of (2.14)) and $\Delta_{N}^{u, \text { sym }}(\mu), \Delta_{N}^{p, \text { sym }}(\mu)$ for any new value of $\mu \in \mathcal{D}$ takes on average 0.31 and 20.99 milliseconds, respectively, which is in total roughly 300 times faster than direct computation of the truth approximation. In the case of Algorithm 3, the same accuracy is achieved for $N_{Z}=35$ (see Fig. 3.11(c)); the Online calculation of $\left(u_{N}(\mu), p_{N}(\mu)\right)$ and $\Delta_{N}^{u, \text { sym }}(\mu), \Delta_{N}^{p, \text { sym }}(\mu)$ then takes on average 0.14 and 13.29 milliseconds, respectively, and is thus roughly 480 times faster than direct computation of the truth approximation. Detailed computation times, also for Algorithm 2, are given in Table 3.4.

(a) Accuracy of at least $1 \%$ (resp., $0.1 \%$ ) for the reduced basis approximations $u_{N}(\mu)$

\begin{tabular}{c|c|c|c|c|c}
\hline Method & $N_{Z}$ & $N$ & $\left(u_{N}(\mu), p_{N}(\mu)\right)$ & $\Delta_{N}^{u, \text { sym }}(\mu), \Delta_{N}^{p, \text { sym }}(\mu)$ & Total \\
\hline Algorithm 1 & $51(78)$ & $17(26)$ & $0.31(0.79)$ & $20.99(39.60)$ & $21.20(40.38)$ \\
\hline Algorithm 2 & $44(61)$ & $19(27)$ & $0.21(0.42)$ & $16.25(24.73)$ & $16.46(25.15)$ \\
\hline Algorithm 3 & $35(55)$ & $13(23)$ & $0.14(0.32)$ & $13.29(21.59)$ & $13.43(21.92)$ \\
\hline
\end{tabular}

(b) Accuracy of at least $1 \%$ (resp., $0.1 \%$ ) for the reduced basis approximations $p_{N}(\mu)$

\begin{tabular}{c|c|c|c|c|c}
\hline Method & $N_{Z}$ & $N$ & $\left(u_{N}(\mu), p_{N}(\mu)\right)$ & $\Delta_{N}^{u, \text { sym }}(\mu), \Delta_{N}^{p, \text { sym }}(\mu)$ & Total \\
\hline Algorithm 1 & $54(84)$ & $18(28)$ & $0.35(0.91)$ & $22.75(44.70)$ & $23.10(45.61)$ \\
\hline Algorithm 2 & $44(72)$ & $19(32)$ & $0.21(0.58)$ & $16.25(31.54)$ & $16.46(32.12)$ \\
\hline Algorithm 3 & $37(62)$ & $14(26)$ & $0.16(0.44)$ & $13.94(25.77)$ & $14.09(26.21)$ \\
\hline
\end{tabular}

Table 3.4.: Average computation times in milliseconds for the Online evaluation of $\left(u_{N}(\mu), p_{N}(\mu)\right)$ (assembly and solution of (2.14)) and the error bounds $\Delta_{N}^{u, \text { sym }}(\mu)$, $\Delta_{N}^{p, \operatorname{sym}}(\mu)$ (see (2.55), (2.56)); times are measured using either Algorithm 1, Algorithm 2 with $\delta_{\text {tol }}^{\beta}=0.1$, or Algorithm 3 with $\delta_{\text {tol }}^{\beta}=0.1$, with a prescribed accuracy of $1 \%$ (resp., $0.1 \%$ ) for the reduced basis approximations (a) $u_{N}(\mu)$ and (b) $p_{N}(\mu)$. 


\subsection{Concluding Remarks}

We present in this chapter numerical results for the reduced basis approach proposed in Chapter 2 applied to a Stokes flow model problem. Compared to earlier approaches, the resulting methods allow us to consider flow problems in parametrized domains with notably greater ease.

The rigorous reduced basis a posteriori error bounds developed in $\S 2.4$ based on Brezzi's theory for saddle point problems are not only computationally more efficient than earlier error estimates based on Babuška's theory for general noncoercive problems, but numerical results demonstrate their superiority also in terms of sharpness; in particular, the bounds provided for the errors in the approximations for the primal variable are reasonably sharp.

Furthermore, numerical results underline theoretical observations in $\S 2.5$ that an enrichment of the reduced basis approximation space with supremizer functions is not necessarily optimal. Adaptively recognizing the need for stabilization, the new sampling procedures for saddle point problems developed in $\S 2.7$ construct reduced basis approximation spaces that provide accurate approximations at much less computational cost: Depending on the employed enrichment strategy, we obtain for our Stokes model problem savings compared to the standard greedy approach of up to $31 \%$. This promises even more significant savings when extending the method to nonlinear problems such as the Navier-Stokes equations.

Considering that our Stokes flow model problem belongs to the symmetric case discussed in Chapter 2, future work may be concerned with a more detailed investigation also of the nonsymmetric case. The exact performance of the adaptive sampling procedures as well as the a posteriori error bounds (with respect to both computational cost and sharpness) are here neither obvious nor precisely predictable; a clarification through further numerical results may thus be of interest. Also, for simplification, our model problem only involves affine geometry variations. As already mentioned in $\S 2.8$, the proposed methods are yet not limited to this case: Techniques as proposed in [7, 40] can be applied, making the proposed approach feasible also, e.g., in the context of nonaffine geometric transformations (see $[19,94])$. 


\section{Chapter 4}

\section{Approximation of Saddle Point Problems by Regularization or Penalty}

\subsection{Introduction}

In the previous Chapters 2 and 3, we discussed the particular difficulties that appear for reduced basis approximations to saddle point problems: Special care must be taken in the construction of stable reduced basis approximation spaces (see §2.5, §2.7, §3.3.2, and §3.3.3), and rigorous reduced basis a posteriori error bounds (more specifically, the required lower bounds to inf-sup stability constants) become increasingly expensive and complicated as geometric variations become more complex (see §2.6.2 and §3.3.4); the latter becomes even more prominent in nonlinear problems such as the Navier-Stokes equations.

This chapter now focuses on the development of rapidly convergent reduced basis approximations and associated rigorous a posteriori error bounds for saddle point problems that arise from a penalty method applied to a constraint problem such as the Stokes or Navier-Stokes equations (see, e.g., $[9,51,52]$ and the references therein). In contrast to reduced basis approaches presented in $[19,63,70,76,88,92,94,104]$ and the methods discussed in Chapter 2, the penalty formulation not only allows us to approximate the primal system variable as well as the Lagrange multiplier but also enables the development of rigorous error bounds for the reduced basis approximation that do not depend on inf-sup stability constants. The expensive evaluation of (lower bounds to) inf-sup constants is thus avoided and more general parametric variations can therefore be considered with relative ease. However, these benefits do not come without expense: The penalty term introduces an $O(\varepsilon)$-error in the "truth" approximations for the primal and Lagrange multiplier variables, where $\varepsilon$ denotes the penalty parameter.

We shall employ the developed methodology in the setting of a perturbed Stokes flow (see Chapter 5). However, we emphasize that saddle point problems as considered in this chapter may appear also in other applications such as nearly incompressible materials (see, e.g., $[9,13,36])$ where they have a physical interpretation.

The chapter is organized as follows: In $\S 4.2$, we introduce the penalty formulation. In $\S 4.3$, we define the reduced basis approximation as the Galerkin projection onto a low-dimensional reduced basis approximation space; we shall also state main properties of the reduced basis system including a priori convergence results. We then develop rigorous a posteriori error 
bounds in $\S 4.4$, and discuss how to construct the low-dimensional reduced basis approximation space in $\S 4.5$; computational efficiency is achieved by the Offline-Online computational strategy summarized in $\S 4.6$. A posteriori error bounds derived in $\S 4.4$ and observations in $\S 4.5$ are again combined within an adaptive sampling procedure briefly explained in $\S 4.7$. Finally, in $\S 4.8$, we provide some concluding remarks.

\subsection{General Problem Statement}

\subsubsection{Formulation}

We assume the setting of a parametrized saddle point problem as introduced in $§ 2.2 .1$. Furthermore, for any parameter $\mu \in \mathcal{D}$, let $c(\cdot, \cdot ; \mu): Y_{\mathrm{e}} \times Y_{\mathrm{e}} \rightarrow \mathbb{R}$ be a bilinear form that is continuous and coercive,

$$
\begin{aligned}
& \gamma_{c}^{\mathrm{e}}(\mu) \equiv \sup _{p \in Y_{\mathrm{e}}} \sup _{q \in Y_{\mathrm{e}}} \frac{c(p, q ; \mu)}{\|p\|_{Y_{\mathrm{e}}}\|q\|_{Y_{\mathrm{e}}}}<\infty, \quad \forall \mu \in \mathcal{D}, \\
& \alpha_{c}^{\mathrm{e}}(\mu) \equiv \inf _{q \in Y_{\mathrm{e}}} \frac{c(q, q ; \mu)}{\|q\|_{Y_{\mathrm{e}}}^{2}}>0, \quad \forall \mu \in \mathcal{D},
\end{aligned}
$$

and which exhibits a $\mu$-affine representation analogous to (2.7). We note that by (4.1) and (4.2), $c(\cdot, \cdot ; \mu)$ provides with $\|\cdot\|_{Y_{\mathrm{e}}, \mu} \equiv \sqrt{c(\cdot, \cdot ; \mu)}$ an energy norm on $Y_{\mathrm{e}}$ that is equivalent to $\|\cdot\|_{Y_{\mathrm{e}}}$ for any $\mu \in \mathcal{D}$.

For a penalty parameter $\varepsilon>0$ that will tend to zero, we now replace the problem (2.6) considered in $\S 2.2 .1$ by a slightly more regular one: Given any $\mu \in \mathcal{D}$, we find $u_{\mathrm{e}}^{\varepsilon}(\mu) \in X_{\mathrm{e}}$ and $p_{\mathrm{e}}^{\varepsilon}(\mu) \in Y_{\mathrm{e}}$ such that

$$
\begin{aligned}
& a\left(u_{\mathrm{e}}^{\varepsilon}(\mu), v ; \mu\right)+b\left(v, p_{\mathrm{e}}^{\varepsilon}(\mu) ; \mu\right)=f(v ; \mu), \quad \forall v \in X_{\mathrm{e}}, \\
& b\left(u_{\mathrm{e}}^{\varepsilon}(\mu), q ; \mu\right)-\varepsilon c\left(p_{\mathrm{e}}^{\varepsilon}(\mu), q ; \mu\right)=g(q ; \mu), \quad \forall q \in Y_{\mathrm{e}} .
\end{aligned}
$$

By the assumptions $(2.1),(2.2),(2.3),(4.1)$, and (4.2), the problem is well-posed and has a unique solution for any $f(\cdot ; \mu) \in X_{\mathrm{e}}^{\prime}$ and $g(\cdot ; \mu) \in Y_{\mathrm{e}}^{\prime}$ (see, e.g., [13]).

Moreover, as we also assumed (2.4), the penalty solution $\left(u_{\mathrm{e}}^{\varepsilon}(\mu), p_{\mathrm{e}}^{\varepsilon}(\mu)\right)$ of (4.3) converges to the solution $\left(u_{\mathrm{e}}(\mu), p_{\mathrm{e}}(\mu)\right)$ of the nonpenalized problem (2.6) as $\varepsilon$ approaches zero. For details, we refer to $[9,101]$ and to the standard texts $[13,36]$. For our specific situation, the following proposition states the main result in this context.

Proposition 4.2.1. For $\varepsilon>0$ and $\mu \in \mathcal{D}$, let $\left(u_{\mathrm{e}}(\mu), p_{\mathrm{e}}(\mu)\right)$ and $\left(u_{\mathrm{e}}^{\varepsilon}(\mu), p_{\mathrm{e}}^{\varepsilon}(\mu)\right)$ denote the solutions to (2.6) and (4.3), respectively. Then, for any $\mu \in \mathcal{D}$, we have

$$
\left\|u_{\mathrm{e}}(\mu)-u_{\mathrm{e}}^{\varepsilon}(\mu)\right\|_{X_{\mathrm{e}}} \leq C_{2}^{\mathrm{e}}(\mu) \varepsilon, \quad\left\|p_{\mathrm{e}}(\mu)-p_{\mathrm{e}}^{\varepsilon}(\mu)\right\|_{Y_{\mathrm{e}}} \leq \frac{\gamma_{a}^{\mathrm{e}}(\mu)}{\beta_{\mathrm{Br}}^{\mathrm{e}}(\mu)} C_{2}^{\mathrm{e}}(\mu) \varepsilon,
$$

where the constant $C_{2}^{\mathrm{e}}(\mu)>0$ depends only on $f(\cdot ; \mu), g(\cdot ; \mu), \gamma_{a}^{\mathrm{e}}(\mu), \alpha_{a}^{\mathrm{e}}(\mu), \gamma_{c}^{\mathrm{e}}(\mu)$, and $\beta_{\mathrm{Br}}^{\mathrm{e}}(\mu)$,

$$
C_{2}^{\mathrm{e}}(\mu) \equiv \frac{\gamma_{a}^{\mathrm{e}}(\mu) \gamma_{c}^{\mathrm{e}}(\mu)}{\alpha_{a}^{\mathrm{e}}(\mu)\left(\beta_{\mathrm{Br}}^{\mathrm{e}}(\mu)\right)^{2}}\left(1+\frac{\gamma_{a}^{\mathrm{e}}(\mu)}{\alpha_{a}^{\mathrm{e}}(\mu)}\right)\left(\|f(\cdot ; \mu)\|_{X_{\mathrm{e}}{ }^{\prime}}+\frac{\gamma_{a}^{\mathrm{e}}(\mu)}{\beta_{\mathrm{Br}}^{\mathrm{e}}(\mu)}\|g(\cdot ; \mu)\|_{Y_{\mathrm{e}}{ }^{\prime}}\right) .
$$


Proof. Take $\varepsilon>0$ and let $\mu$ be any parameter in $\mathcal{D}$. It follows from (2.6) and (4.3) that

$$
\begin{aligned}
a\left(u_{\mathrm{e}}(\mu)-u_{\mathrm{e}}^{\varepsilon}(\mu), v ; \mu\right)+b\left(v, p_{\mathrm{e}}(\mu)-p_{\mathrm{e}}^{\varepsilon}(\mu) ; \mu\right) & =0, & & \forall v \in X_{\mathrm{e}}, \\
b\left(u_{\mathrm{e}}(\mu)-u_{\mathrm{e}}^{\varepsilon}(\mu), q ; \mu\right)-\varepsilon c\left(p_{\mathrm{e}}(\mu)-p_{\mathrm{e}}^{\varepsilon}(\mu), q ; \mu\right) & =-\varepsilon c\left(p_{\mathrm{e}}(\mu), q ; \mu\right), & & \forall q \in Y_{\mathrm{e}} .
\end{aligned}
$$

First, combining the inf-sup condition (2.4) with (4.4) and (2.1), we obtain that

$$
\begin{aligned}
\beta_{\mathrm{Br}}^{\mathrm{e}}(\mu)\left\|p_{\mathrm{e}}(\mu)-p_{\mathrm{e}}^{\varepsilon}(\mu)\right\|_{Y_{\mathrm{e}}} & \leq \sup _{v \in X_{\mathrm{e}}} \frac{b\left(v, p_{\mathrm{e}}(\mu)-p_{\mathrm{e}}^{\varepsilon}(\mu) ; \mu\right)}{\|v\|_{X_{\mathrm{e}}}}=\sup _{v \in X_{\mathrm{e}}} \frac{-a\left(u_{\mathrm{e}}(\mu)-u_{\mathrm{e}}^{\varepsilon}(\mu), v ; \mu\right)}{\|v\|_{X_{\mathrm{e}}}} \\
& \leq \gamma_{a}^{\mathrm{e}}(\mu)\left\|u_{\mathrm{e}}(\mu)-u_{\mathrm{e}}^{\varepsilon}(\mu)\right\|_{X_{\mathrm{e}}} .
\end{aligned}
$$

Second, setting $v=u_{\mathrm{e}}(\mu)-u_{\mathrm{e}}^{\varepsilon}(\mu), q=p_{\mathrm{e}}(\mu)-p_{\mathrm{e}}^{\varepsilon}(\mu)$ in (4.4), (4.5) and subtracting the second from the first equation yields

$$
\left\|u_{\mathrm{e}}(\mu)-u_{\mathrm{e}}^{\varepsilon}(\mu)\right\|_{X_{\mathrm{e}}, \mu}^{2}+\varepsilon\left\|p_{\mathrm{e}}(\mu)-p_{\mathrm{e}}^{\varepsilon}(\mu)\right\|_{Y_{\mathrm{e}}, \mu}^{2}=\varepsilon c\left(p_{\mathrm{e}}(\mu), p_{\mathrm{e}}(\mu)-p_{\mathrm{e}}^{\varepsilon}(\mu) ; \mu\right),
$$

which provides by (2.3), (4.2), and (4.1) that

$$
\alpha_{a}^{\mathrm{e}}(\mu)\left\|u_{\mathrm{e}}(\mu)-u_{\mathrm{e}}^{\varepsilon}(\mu)\right\|_{X_{\mathrm{e}}}^{2} \leq \varepsilon \gamma_{c}^{\mathrm{e}}(\mu)\left\|p_{\mathrm{e}}(\mu)\right\|_{Y_{\mathrm{e}}}\left\|p_{\mathrm{e}}(\mu)-p_{\mathrm{e}}^{\varepsilon}(\mu)\right\|_{Y_{\mathrm{e}}} .
$$

We may then apply (4.6) and divide by $\alpha_{a}^{\mathrm{e}}(\mu)\left\|u_{\mathrm{e}}(\mu)-u_{\mathrm{e}}^{\varepsilon}(\mu)\right\|_{X_{\mathrm{e}}}$ such that

$$
\left\|u_{\mathrm{e}}(\mu)-u_{\mathrm{e}}^{\varepsilon}(\mu)\right\|_{X_{\mathrm{e}}} \leq \frac{\gamma_{a}^{\mathrm{e}}(\mu) \gamma_{c}^{\mathrm{e}}(\mu)}{\alpha_{a}^{\mathrm{e}}(\mu) \beta_{\mathrm{Br}}^{\mathrm{e}}(\mu)}\left\|p_{\mathrm{e}}(\mu)\right\|_{Y_{\mathrm{e}}} \varepsilon
$$

The statement then follows from (4.6), (4.8), and the stability estimate (see [13], cf. (2.38))

$$
\left\|p_{\mathrm{e}}(\mu)\right\|_{Y_{\mathrm{e}}} \leq \frac{1}{\beta_{\mathrm{Br}}^{\mathrm{e}}(\mu)}\left(1+\frac{\gamma_{a}^{\mathrm{e}}(\mu)}{\alpha_{a}^{\mathrm{e}}(\mu)}\right)\left(\|f(\cdot ; \mu)\|_{X_{\mathrm{e}}{ }^{\prime}}+\frac{\gamma_{a}^{\mathrm{e}}(\mu)}{\beta_{\mathrm{Br}}^{\mathrm{e}}(\mu)}\|g(\cdot ; \mu)\|_{Y_{\mathrm{e}}{ }^{\prime}}\right) .
$$

In the special case that $a(\cdot, \cdot ; \mu)$ is symmetric for all $\mu \in \mathcal{D}$, the $\varepsilon$-independent constants given in Proposition 4.2.1 can be sharpened.

Proposition 4.2.2. Let $a(\cdot, \cdot ; \mu)$ be symmetric for all $\mu \in \mathcal{D}$. For $\varepsilon>0$ and $\mu \in \mathcal{D}$, let $\left(u_{\mathrm{e}}(\mu), p_{\mathrm{e}}(\mu)\right)$ and $\left(u_{\mathrm{e}}^{\varepsilon}(\mu), p_{\mathrm{e}}^{\varepsilon}(\mu)\right)$ denote the solutions to (2.6) and (4.3), respectively. Then, for any $\mu \in \mathcal{D}$, we have

$$
\left\|u_{\mathrm{e}}(\mu)-u_{\mathrm{e}}^{\varepsilon}(\mu)\right\|_{X_{\mathrm{e}}} \leq \frac{C_{2}^{\mathrm{e}, \mathrm{sym}}(\mu)}{\sqrt{\alpha_{a}^{\mathrm{e}}(\mu)}} \varepsilon, \quad\left\|p_{\mathrm{e}}(\mu)-p_{\mathrm{e}}^{\varepsilon}(\mu)\right\|_{Y_{\mathrm{e}}} \leq \frac{\sqrt{\gamma_{a}^{\mathrm{e}}(\mu)}}{\beta_{\mathrm{Br}}^{\mathrm{e}}(\mu)} C_{2}^{\mathrm{e}, \mathrm{sym}}(\mu) \varepsilon,
$$

where the constant $C_{2}^{\mathrm{e}, \mathrm{sym}}(\mu)>0$ may be expressed as

$$
C_{2}^{\mathrm{e}, \mathrm{sym}}(\mu) \equiv \frac{\sqrt{\gamma_{a}^{\mathrm{e}}(\mu)} \gamma_{c}^{\mathrm{e}}(\mu)}{\left(\beta_{\mathrm{Br}}^{\mathrm{e}}(\mu)\right)^{2}}\left[\left(1+\sqrt{\frac{\gamma_{a}^{\mathrm{e}}(\mu)}{\alpha_{a}^{\mathrm{e}}(\mu)}}\right)\|f(\cdot ; \mu)\|_{X_{\mathrm{e}}{ }^{\prime}}+\frac{\gamma_{a}^{\mathrm{e}}(\mu)}{\beta_{\mathrm{Br}}^{\mathrm{e}}(\mu)}\|g(\cdot ; \mu)\|_{Y_{\mathrm{e}}{ }^{\prime}}\right]
$$


Proof. We recall that, as a continuous, symmetric, and coercive bilinear form, $a(\cdot, \cdot ; \mu)$ defines an inner product on $X_{\mathrm{e}}$ for any $\mu \in \mathcal{D}$. Therefore, following the steps presented in the previous proof, we now obtain that

$$
\beta_{\mathrm{Br}}^{\mathrm{e}}(\mu)\left\|p_{\mathrm{e}}(\mu)-p_{\mathrm{e}}^{\varepsilon}(\mu)\right\|_{Y_{\mathrm{e}}} \leq \sqrt{\gamma_{a}^{\mathrm{e}}(\mu)}\left\|u_{\mathrm{e}}(\mu)-u_{\mathrm{e}}^{\varepsilon}(\mu)\right\|_{X_{\mathrm{e}}, \mu}
$$

from the Cauchy-Schwarz inequality for the inner product $a(\cdot, \cdot ; \mu)$. Using (4.7), (4.2), (4.1), and (4.9) then yields

$$
\left\|u_{\mathrm{e}}(\mu)-u_{\mathrm{e}}^{\varepsilon}(\mu)\right\|_{X_{\mathrm{e}}, \mu} \leq \sqrt{\gamma_{a}^{\mathrm{e}}(\mu)} \frac{\gamma_{c}^{\mathrm{e}}(\mu)}{\beta_{\mathrm{Br}}^{\mathrm{e}}(\mu)}\left\|p_{\mathrm{e}}(\mu)\right\|_{Y_{\mathrm{e}}} \varepsilon,
$$

and the statement follows from combining (4.10) and the stability estimate (cf. (2.56))

$$
\left\|p_{\mathrm{e}}(\mu)\right\|_{Y_{\mathrm{e}}} \leq \frac{1}{\beta_{\mathrm{Br}}^{\mathrm{e}}(\mu)}\left[\left(1+\sqrt{\frac{\gamma_{a}^{\mathrm{e}}(\mu)}{\alpha_{a}^{\mathrm{e}}(\mu)}}\right)\|f(\cdot ; \mu)\|_{X_{\mathrm{e}^{\prime}}}+\frac{\gamma_{a}^{\mathrm{e}}(\mu)}{\beta_{\mathrm{Br}}^{\mathrm{e}}(\mu)}\|g(\cdot ; \mu)\|_{Y_{\mathrm{e}}{ }^{\prime}}\right]
$$

with either (2.3) or (4.9).

\subsubsection{Truth Approximation}

As in $\S 2.2 .2$, we denote by $X$ and $Y$ finite-dimensional subspaces of $X_{\mathrm{e}}$ and $Y_{\mathrm{e}}$ satisfying $(2.8),(2.9),(2.10)$, and (2.11). These in particular also inherit the continuity and coercivity properties of $c(\cdot, \cdot ; \mu)$,

$$
\begin{aligned}
& \gamma_{c}(\mu) \equiv \sup _{p \in Y} \sup _{q \in Y} \frac{c(p, q ; \mu)}{\|p\|_{Y}\|q\|_{Y}}<\infty, \quad \forall \mu \in \mathcal{D}, \\
& \alpha_{c}(\mu) \equiv \inf _{q \in Y} \frac{c(q, q ; \mu)}{\|q\|_{Y}^{2}}>0, \quad \forall \mu \in \mathcal{D} ;
\end{aligned}
$$

for any $\mu \in \mathcal{D},\|\cdot\|_{Y, \mu} \equiv\|\cdot\|_{Y_{\mathrm{e}}, \mu}$ thus defines a norm on $Y$ that is equivalent to $\|\cdot\|_{Y}$. Again, we denote by $\mathcal{N}$ the large dimension of the approximation space $Z=X \times Y$.

We now define the "truth" approximation as follows: For a sufficiently small penalty parameter $\varepsilon>0$ and any given $\mu \in \mathcal{D}$, we look for $u^{\varepsilon}(\mu) \in X$ and $p^{\varepsilon}(\mu) \in Y$ such that

$$
\begin{aligned}
a\left(u^{\varepsilon}(\mu), v ; \mu\right)+b\left(v, p^{\varepsilon}(\mu) ; \mu\right) & =f(v ; \mu), & & \forall v \in X, \\
b\left(u^{\varepsilon}(\mu), q ; \mu\right)-\varepsilon c\left(p^{\varepsilon}(\mu), q ; \mu\right) & =g(q ; \mu), & & \forall q \in Y .
\end{aligned}
$$

As for the exact problem in $\S 4.2 .1$, it follows from (2.8), (2.9), (2.10), (4.11), and (4.12) that the "truth" problem (4.13) is well-posed and has a unique solution for any $f(\cdot ; \mu) \in X_{\mathrm{e}}^{\prime}$ and $g(\cdot ; \mu) \in Y_{\mathrm{e}}^{\prime}$. The system (4.13) may be equivalently written as

$$
\begin{aligned}
& A(\mu) u^{\varepsilon}(\mu)+B(\mu)^{t} p^{\varepsilon}(\mu)=f(\mu) \text { in } X^{\prime}, \\
& B(\mu) u^{\varepsilon}(\mu)-\varepsilon C(\mu) p^{\varepsilon}(\mu)=g(\mu) \text { in } Y^{\prime} \text {, }
\end{aligned}
$$

where $A(\mu), B(\mu), B(\mu)^{t}, f(\mu)$, and $g(\mu)$ are defined as in $\S 2.2 .2$, and the bounded linear operator $C(\mu): Y \rightarrow Y^{\prime}$ is given by

$$
\langle C(\mu) p, q\rangle=c(p, q ; \mu), \quad \forall p, q \in Y .
$$


Since $X$ and $Y$ are moreover assumed to satisfy the LBB inf-sup condition (2.11), the penalty solution $\left(u^{\varepsilon}(\mu), p^{\varepsilon}(\mu)\right)$ of $(4.13)$ converges to the solution $(u(\mu), p(\mu))$ of the nonpenalized problem (2.13) as $\varepsilon$ approaches zero; more precisely, corresponding to Proposition 4.2.1 and Proposition 4.2.2, the following results hold true.

Proposition 4.2.3. For $\varepsilon>0$ and $\mu \in \mathcal{D}$, let $(u(\mu), p(\mu))$ and $\left(u^{\varepsilon}(\mu), p^{\varepsilon}(\mu)\right)$ denote the solutions to (2.13) and (4.13), respectively. Then, for any $\mu \in \mathcal{D}$, we have

$$
\left\|u(\mu)-u^{\varepsilon}(\mu)\right\|_{X} \leq C_{2}(\mu) \varepsilon, \quad\left\|p(\mu)-p^{\varepsilon}(\mu)\right\|_{Y} \leq \frac{\gamma_{a}(\mu)}{\beta_{\mathrm{Br}}(\mu)} C_{2}(\mu) \varepsilon
$$

where the constant $C_{2}(\mu)>0$ depends only on $f(\cdot ; \mu), g(\cdot ; \mu), \gamma_{a}(\mu), \alpha_{a}(\mu), \gamma_{c}(\mu)$, and $\beta_{\mathrm{Br}}(\mu)$,

$$
C_{2}(\mu) \equiv \frac{\gamma_{a}(\mu) \gamma_{c}(\mu)}{\alpha_{a}(\mu)\left(\beta_{\mathrm{Br}}(\mu)\right)^{2}}\left(1+\frac{\gamma_{a}(\mu)}{\alpha_{a}(\mu)}\right)\left(\|f(\cdot ; \mu)\|_{X^{\prime}}+\frac{\gamma_{a}(\mu)}{\beta_{\mathrm{Br}}(\mu)}\|g(\cdot ; \mu)\|_{Y^{\prime}}\right)
$$

Proof. Replacing the spaces $X_{\mathrm{e}}$ and $Y_{\mathrm{e}}$ with $X$ and $Y$, respectively, this is a direct application of Proposition 4.2.1.

Proposition 4.2.4. Let $a(\cdot, \cdot ; \mu)$ be symmetric for all $\mu \in \mathcal{D}$. For $\varepsilon>0$ and $\mu \in \mathcal{D}$, let $(u(\mu), p(\mu))$ and $\left(u^{\varepsilon}(\mu), p^{\varepsilon}(\mu)\right)$ denote the solutions to (2.13) and (4.13), respectively. Then, for any $\mu \in \mathcal{D}$, we have

$$
\left\|u(\mu)-u^{\varepsilon}(\mu)\right\|_{X} \leq \frac{C_{2}^{\mathrm{sym}}(\mu)}{\sqrt{\alpha_{a}(\mu)}} \varepsilon, \quad\left\|p(\mu)-p^{\varepsilon}(\mu)\right\|_{Y} \leq \frac{\sqrt{\gamma_{a}(\mu)}}{\beta_{\mathrm{Br}}(\mu)} C_{2}^{\mathrm{sym}}(\mu) \varepsilon,
$$

where the constant $C_{2}^{\mathrm{sym}}(\mu)>0$ may be expressed as

$$
C_{2}^{\mathrm{sym}}(\mu) \equiv \frac{\sqrt{\gamma_{a}(\mu)} \gamma_{c}(\mu)}{\left(\beta_{\mathrm{Br}}(\mu)\right)^{2}}\left[\left(1+\sqrt{\frac{\gamma_{a}(\mu)}{\alpha_{a}(\mu)}}\right)\|f(\cdot ; \mu)\|_{X^{\prime}}+\frac{\gamma_{a}(\mu)}{\beta_{\mathrm{Br}}(\mu)}\|g(\cdot ; \mu)\|_{Y^{\prime}}\right] \text {. }
$$

Proof. Replacing the spaces $X_{\mathrm{e}}$ and $Y_{\mathrm{e}}$ with $X$ and $Y$, respectively, this is a direct application of Proposition 4.2.2.

We shall build our reduced basis approximation upon the "truth" discretization (4.13), and we shall measure the error in our reduced basis prediction relative to $\left(u^{\varepsilon}(\mu), p^{\varepsilon}(\mu)\right)$.

\subsection{Reduced Basis Approximation}

We now turn to the reduced basis method, discussing the approximation procedure, rigorous a posteriori error estimators, and the effects of the penalty parameter $\varepsilon$. 


\subsubsection{Formulation}

Suppose that we are given a set of nested, low-dimensional reduced basis approximation subspaces $X_{N} \subset X_{N+1} \subset X$ and $Y_{N} \subset Y_{N+1} \subset Y, N \in \mathbb{N}_{\max } \equiv\left\{1, \ldots, N_{\max }\right\}$, then the reduced basis approximation is again defined as the Galerkin projection with respect to the truth problem (4.13) onto these low-dimensional subspaces: For $\varepsilon>0$ sufficiently small and any given $\mu \in \mathcal{D}$, we find $u_{N}^{\varepsilon}(\mu) \in X_{N}$ and $p_{N}^{\varepsilon}(\mu) \in Y_{N}$ such that

$$
\begin{array}{rlrl}
a\left(u_{N}^{\varepsilon}(\mu), v_{N} ; \mu\right)+b\left(v_{N}, p_{N}^{\varepsilon}(\mu) ; \mu\right) & =f\left(v_{N} ; \mu\right), & & \forall v_{N} \in X_{N}, \\
b\left(u_{N}^{\varepsilon}(\mu), q_{N} ; \mu\right)-\varepsilon c\left(p_{N}^{\varepsilon}(\mu), q_{N} ; \mu\right) & =g\left(q_{N} ; \mu\right), & \forall q_{N} \in Y_{N} .
\end{array}
$$

Written in operator notation, the discrete system reads

$$
\begin{array}{cl}
A_{N}(\mu) u_{N}^{\varepsilon}(\mu)+B_{N}(\mu)^{t} p_{N}^{\varepsilon}(\mu)=f_{N}(\mu) & \text { in } X_{N}^{\prime}, \\
B_{N}(\mu) u_{N}^{\varepsilon}(\mu)-\varepsilon C_{N}(\mu) p_{N}^{\varepsilon}(\mu)=g_{N}(\mu) & \text { in } Y_{N}^{\prime},
\end{array}
$$

where $A_{N}(\mu), B_{N}(\mu), B_{N}(\mu)^{t}, f_{N}(\mu)$, and $g_{N}(\mu)$ are defined as in $\S 2.3 .1$, and the operator $C_{N}(\mu): Y_{N} \rightarrow Y_{N}^{\prime}$ is given by

$$
\left\langle C_{N}(\mu) p_{N}, q_{N}\right\rangle=c\left(p_{N}, q_{N} ; \mu\right), \quad \forall p_{N}, q_{N} \in Y_{N} .
$$

\subsubsection{Properties of the Discrete System}

The introduction of the penalty term regularizes the reduced basis problem such that no further requirements for the reduced basis approximation spaces $X_{N}$ and $Y_{N}$ are needed for (4.14) to be well-posed. Let $\varepsilon>0$ and $\mu \in \mathcal{D}$. As the bilinear form $c(\cdot, \cdot ; \mu)$ satisfies (4.11) and (4.12), the operator $C_{N}(\mu)$ is nonsingular and (4.16) is equivalent to

$$
p_{N}^{\varepsilon}(\mu)=\frac{1}{\varepsilon} C_{N}(\mu)^{-1}\left(B_{N}(\mu) u_{N}^{\varepsilon}(\mu)-g_{N}(\mu)\right) .
$$

Inserting this in (4.15), $u_{N}^{\varepsilon}(\mu)$ is given as the unique solution to the coercive problem

$$
\left(A_{N}(\mu)+\frac{1}{\varepsilon} B_{N}(\mu)^{t} C_{N}(\mu)^{-1} B_{N}(\mu)\right) u_{N}^{\varepsilon}(\mu)=f_{N}(\mu)+\frac{1}{\varepsilon} B_{N}(\mu)^{t} C_{N}(\mu)^{-1} g_{N}(\mu) \text { in } X_{N}^{\prime},
$$

and $p_{N}^{\varepsilon}(\mu)$ is clearly obtained through (4.17). In contrast to our observations for (2.14) in $\S 2.3 .2$ where the inf-sup condition (2.17) represents an additional requirement for $X_{N}, Y_{N}$ that needs to be satisfied, the system (4.14) is thus uniquely solvable for any parameter $\mu \in \mathcal{D}$ for any choice of $X_{N}, Y_{N}$.

\subsubsection{A Priori Error Estimation}

We here consider a priori estimates for the errors in our reduced basis approximations.

For any $\varepsilon>0$ and $\mu \in \mathcal{D}$, we denote the errors in the reduced basis approximations $u_{N}^{\varepsilon}(\mu) \in X_{N}, p_{N}^{\varepsilon}(\mu) \in Y_{N}$, and $\left(u_{N}^{\varepsilon}(\mu), p_{N}^{\varepsilon}(\mu)\right) \in Z_{N}=X_{N} \times Y_{N}$ with respect to the truth penalty approximations by

$$
\begin{aligned}
e_{N}^{u, \varepsilon}(\mu) & \equiv u^{\varepsilon}(\mu)-u_{N}^{\varepsilon}(\mu) \in X, \\
e_{N}^{p, \varepsilon}(\mu) & \equiv p^{\varepsilon}(\mu)-p_{N}^{\varepsilon}(\mu) \in Y, \\
e_{N}^{\varepsilon}(\mu) & \equiv\left(e_{N}^{u, \varepsilon}(\mu), e_{N}^{p, \varepsilon}(\mu)\right) \in Z .
\end{aligned}
$$


The total error $e_{N}^{\varepsilon}(\mu)$ shall be measured in the $\varepsilon$-dependent energy norm $\|\cdot\|_{Z, \varepsilon, \mu}$ given on the combined space $Z=X \times Y$ by

$$
\|(v, q)\|_{Z, \varepsilon, \mu} \equiv(a(v, v ; \mu)+\varepsilon c(q, q ; \mu))^{1 / 2}=\left(\|v\|_{X, \mu}^{2}+\varepsilon\|q\|_{Y, \mu}^{2}\right)^{1 / 2}, \quad \forall(v, q) \in Z .
$$

From $(2.8),(2.10),(4.11)$, and (4.12), this norm is clearly equivalent to $\|\cdot\|_{Z}$; however, we note that this relationship depends on the penalty parameter $\varepsilon$,

$$
\sqrt{\min \left\{\alpha_{a}(\mu), \varepsilon \alpha_{c}(\mu)\right\}}\|\cdot\|_{Z} \leq\|\cdot\|_{Z, \varepsilon, \mu} \leq \sqrt{\max \left\{\gamma_{a}(\mu), \varepsilon \gamma_{c}(\mu)\right\}}\|\cdot\|_{Z}, \quad \forall \mu \in \mathcal{D} .
$$

Proposition 4.3.1. For $\varepsilon>0, \mu \in \mathcal{D}$, and $N \in \mathbb{N}_{\max }$, we have

$$
\left\|e_{N}^{\varepsilon}(\mu)\right\|_{Z, \varepsilon, \mu} \leq C\left(\frac{1}{\sqrt{\varepsilon}}, \mu\right) \inf _{\left(v_{N}, q_{N}\right) \in Z_{N}}\left\|\left(u^{\varepsilon}(\mu), p^{\varepsilon}(\mu)\right)-\left(v_{N}, q_{N}\right)\right\|_{Z, \varepsilon, \mu}
$$

where the constant $C\left(\frac{1}{\sqrt{\varepsilon}}, \mu\right)>0$ may be expressed as

$$
C\left(\frac{1}{\sqrt{\varepsilon}}, \mu\right) \equiv 1+\max \left\{\frac{\gamma_{a}(\mu)}{\alpha_{a}(\mu)}, \frac{\gamma_{c}(\mu)}{\alpha_{c}(\mu)}\right\}+\frac{\gamma_{b}(\mu)}{\sqrt{\alpha_{a}(\mu) \alpha_{c}(\mu) \varepsilon}} .
$$

Proof. Let $\varepsilon>0, \mu \in \mathcal{D}$, and $N \in \mathbb{N}_{\max }$. (We suppress the argument $\mu$ in this proof for clarity of exposition.) For any $v_{N} \in X_{N}$ and $q_{N} \in Y_{N}$, it follows from (4.13) and (4.14) that

$$
\begin{array}{ll}
a\left(v_{N}-u_{N}^{\varepsilon}, w_{N}\right)+b\left(w_{N}, q_{N}-p_{N}^{\varepsilon}\right)=a\left(v_{N}-u^{\varepsilon}, w_{N}\right)+b\left(w_{N}, q_{N}-p^{\varepsilon}\right), & \forall w_{N} \in X_{N}, \\
b\left(v_{N}-u_{N}^{\varepsilon}, r_{N}\right)-\varepsilon c\left(q_{N}-p_{N}^{\varepsilon}, r_{N}\right)=b\left(v_{N}-u^{\varepsilon}, r_{N}\right)-\varepsilon c\left(q_{N}-p^{\varepsilon}, r_{N}\right), & \forall r_{N} \in Y_{N} .
\end{array}
$$

Setting here $w_{N}=v_{N}-u_{N}^{\varepsilon} \in X_{N}, r_{N}=q_{N}-p_{N}^{\varepsilon} \in Y_{N}$ and subtracting the second from the first equation yields

$$
\begin{aligned}
\left\|\left(v_{N}-u_{N}^{\varepsilon}, q_{N}-p_{N}^{\varepsilon}\right)\right\|_{Z, \varepsilon, \mu}^{2}= & \left\|v_{N}-u_{N}^{\varepsilon}\right\|_{X, \mu}^{2}+\varepsilon\left\|q_{N}-p_{N}^{\varepsilon}\right\|_{Y, \mu}^{2} \\
= & a\left(v_{N}-u^{\varepsilon}, v_{N}-u_{N}^{\varepsilon}\right)+b\left(v_{N}-u_{N}^{\varepsilon}, q_{N}-p^{\varepsilon}\right) \\
& \quad-b\left(v_{N}-u^{\varepsilon}, q_{N}-p_{N}^{\varepsilon}\right)+\varepsilon c\left(q_{N}-p^{\varepsilon}, q_{N}-p_{N}^{\varepsilon}\right),
\end{aligned}
$$

which, from (2.8), (2.9), (2.10), (4.11), and (4.12), can be estimated by

$$
\begin{aligned}
\leq \frac{\gamma_{a}}{\alpha_{a}}\left\|v_{N}-u^{\varepsilon}\right\|_{X, \mu}\left\|v_{N}-u_{N}^{\varepsilon}\right\|_{X, \mu}+\frac{\gamma_{b}}{\sqrt{\alpha_{a} \alpha_{c}}}\left\|v_{N}-u_{N}^{\varepsilon}\right\|_{X, \mu}\left\|q_{N}-p^{\varepsilon}\right\|_{Y, \mu} \\
\quad+\frac{\gamma_{b}}{\sqrt{\alpha_{a} \alpha_{c}}}\left\|v_{N}-u^{\varepsilon}\right\|_{X, \mu}\left\|q_{N}-p_{N}^{\varepsilon}\right\|_{Y, \mu}+\varepsilon \frac{\gamma_{c}}{\alpha_{c}}\left\|q_{N}-p^{\varepsilon}\right\|_{Y, \mu}\left\|q_{N}-p_{N}^{\varepsilon}\right\|_{Y, \mu} .
\end{aligned}
$$

Written in matrix-vector notation, this reads

$$
\left\|\left(v_{N}-u_{N}^{\varepsilon}, q_{N}-p_{N}^{\varepsilon}\right)\right\|_{Z, \varepsilon, \mu}^{2} \leq\left(\begin{array}{c}
\left\|v_{N}-u_{N}^{\varepsilon}\right\|_{X, \mu} \\
\sqrt{\varepsilon}\left\|q_{N}-p_{N}^{\varepsilon}\right\|_{Y, \mu}
\end{array}\right)^{t}\left(\begin{array}{cc}
\frac{\gamma_{a}}{\alpha_{a}} & \frac{\gamma_{b}}{\sqrt{\alpha_{a} \alpha_{c} \varepsilon}} \\
\frac{\gamma_{b}}{\sqrt{\alpha_{a} \alpha_{c} \varepsilon}} & \frac{\gamma_{c}}{\alpha_{c}}
\end{array}\right)\left(\begin{array}{c}
\left\|v_{N}-u^{\varepsilon}\right\|_{X, \mu} \\
\sqrt{\varepsilon}\left\|q_{N}-p^{\varepsilon}\right\|_{Y, \mu}
\end{array}\right),
$$

which is further bounded by

$$
\leq\left(\max \left\{\frac{\gamma_{a}}{\alpha_{a}}, \frac{\gamma_{c}}{\alpha_{c}}\right\}+\frac{\gamma_{b}}{\sqrt{\alpha_{a} \alpha_{c} \varepsilon}}\right)\left\|\left(v_{N}-u_{N}^{\varepsilon}, q_{N}-p_{N}^{\varepsilon}\right)\right\|_{Z, \varepsilon, \mu}\left\|\left(v_{N}-u^{\varepsilon}, q_{N}-p^{\varepsilon}\right)\right\|_{Z, \varepsilon, \mu}
$$


from the same techniques as used in the proof of Corollary 2.4.3. We finally have

$$
\|\left(v_{N}-u_{N}^{\varepsilon}, q_{N}-p_{N}^{\varepsilon}\left\|_{Z, \varepsilon, \mu} \leq\left(\max \left\{\frac{\gamma_{a}}{\alpha_{a}}, \frac{\gamma_{c}}{\alpha_{c}}\right\}+\frac{\gamma_{b}}{\sqrt{\alpha_{a} \alpha_{c} \varepsilon}}\right)\right\|\left(u^{\varepsilon}-v_{N}, p^{\varepsilon}-q_{N}\right) \|_{Z, \varepsilon, \mu}\right.
$$

for all $v_{N} \in X_{N}, q_{N} \in Y_{N}$, and (4.20) follows from the triangle inequality

$$
\begin{aligned}
\left\|e_{N}^{\varepsilon}\right\|_{Z, \varepsilon, \mu} & =\left\|\left(u^{\varepsilon}-u_{N}^{\varepsilon}, p^{\varepsilon}-p_{N}^{\varepsilon}\right)\right\|_{Z, \varepsilon, \mu} \\
& \leq\left\|\left(u^{\varepsilon}-v_{N}, p^{\varepsilon}-q_{N}\right)\right\|_{Z, \varepsilon, \mu}+\left\|\left(v_{N}-u_{N}^{\varepsilon}, q_{N}-p_{N}^{\varepsilon}\right)\right\|_{Z, \varepsilon, \mu}
\end{aligned}
$$

and (4.23).

In the case of a symmetric problem (4.3), the constant given in (4.20) can be improved; we may then derive the following a priori error estimate.

Proposition 4.3.2. Let $a(\cdot, \cdot ; \mu)$ and $c(\cdot, \cdot ; \mu)$ be symmetric for all $\mu \in \mathcal{D}$. For $\varepsilon>0, \mu \in \mathcal{D}$, and $N \in \mathbb{N}_{\max }$, we then have

$$
\left\|e_{N}^{\varepsilon}(\mu)\right\|_{Z, \varepsilon, \mu} \leq\left(2+\frac{\gamma_{b}(\mu)}{\sqrt{\alpha_{a}(\mu) \alpha_{c}(\mu) \varepsilon}}\right) \inf _{\left(v_{N}, q_{N}\right) \in Z_{N}}\left\|\left(u^{\varepsilon}(\mu), p^{\varepsilon}(\mu)\right)-\left(v_{N}, q_{N}\right)\right\|_{Z, \varepsilon, \mu} .
$$

Proof. As continuous and symmetric bilinear forms satisfying (2.10) and (4.12), $a(\cdot, \cdot ; \mu)$ and $c(\cdot, \cdot ; \mu)$ define inner products on $X$ and $Y$, respectively, for any parameter $\mu \in \mathcal{D}$. Therefore, proceeding as in the previous proof, we may now apply the Cauchy-Schwarz inequality in (4.21) that yields

$$
\begin{aligned}
& \left\|\left(v_{N}-u_{N}^{\varepsilon}, q_{N}-p_{N}^{\varepsilon}\right)\right\|_{Z, \varepsilon, \mu}^{2} \\
& \leq\left\|v_{N}-u^{\varepsilon}\right\|_{X, \mu}\left\|v_{N}-u_{N}^{\varepsilon}\right\|_{X, \mu}+\frac{\gamma_{b}}{\sqrt{\alpha_{a} \alpha_{c}}}\left\|v_{N}-u_{N}^{\varepsilon}\right\|_{X, \mu}\left\|q_{N}-p^{\varepsilon}\right\|_{Y, \mu} \\
& \quad+\frac{\gamma_{b}}{\sqrt{\alpha_{a} \alpha_{c}}}\left\|v_{N}-u^{\varepsilon}\right\|_{X, \mu}\left\|q_{N}-p_{N}^{\varepsilon}\right\|_{Y, \mu}+\varepsilon\left\|q_{N}-p^{\varepsilon}\right\|_{Y, \mu}\left\|q_{N}-p_{N}^{\varepsilon}\right\|_{Y, \mu}
\end{aligned}
$$

instead of (4.22). The statement then follows from analogous steps as presented above.

Through the introduction of the penalty term, we thus obtain a priori error estimates that do not depend on inf-sup constants. However, we note that they depend on the penalty parameter $\varepsilon$ : The rate at which the reduced basis approximation $\left(u_{N}^{\varepsilon}(\mu), p_{N}^{\varepsilon}(\mu)\right)$ converges to the truth solution $\left(u^{\varepsilon}(\mu), p^{\varepsilon}(\mu)\right)$ behaves as $O\left(\frac{1}{\sqrt{\varepsilon}}\right)$ and thus may be poor for $\varepsilon$ small if no further precautions are taken. Sufficient precautions are, for example, to construct the reduced basis approximation spaces $X_{N}, Y_{N}$ such that they are stable as defined in $\S 2.3 .2$; in this case, there moreover holds the following result.

Proposition 4.3.3. If the spaces $X_{N}, Y_{N}$ are stable (see §2.3.2), we have for $\varepsilon \in(0,1)$, $\mu \in \mathcal{D}$, and $N \in \mathbb{N}_{\max }$,

$$
\left\|e_{N}^{\varepsilon}(\mu)\right\|_{Z} \leq C(\mu) \inf _{\left(v_{N}, q_{N}\right) \in Z_{N}}\left\|\left(u^{\varepsilon}(\mu), p^{\varepsilon}(\mu)\right)-\left(v_{N}, q_{N}\right)\right\|_{Z},
$$

where the constant $C(\mu)>0$ depends only on $\gamma_{a}(\mu), \alpha_{a}(\mu), \gamma_{c}(\mu), \alpha_{c}(\mu), \gamma_{b}(\mu)$, and $\beta_{N}(\mu)$. 
Proof. We here essentially combine the approach in the proof of Proposition 4.3.1 with techniques used for Proposition 2.3.2. Let $\varepsilon>0, \mu \in \mathcal{D}$, and $N \in \mathbb{N}_{\max }$. (We suppress all $\mu$-dependence in this proof for clarity of exposition.)

Take any $v_{N} \in X_{N}$ and $q_{N} \in Y_{N}$. We start as in the proof of Proposition 4.3.1 and obtain (4.21). From (2.8), (2.10), (4.11), (4.12), and (2.9), it then follows that

$$
\begin{aligned}
& \alpha_{a}\left\|v_{N}-u_{N}^{\varepsilon}\right\|_{X}^{2}+\varepsilon \alpha_{c}\left\|q_{N}-p_{N}^{\varepsilon}\right\|_{Y}^{2} \\
& \quad \leq \gamma_{a}\left\|v_{N}-u^{\varepsilon}\right\|_{X}\left\|v_{N}-u_{N}^{\varepsilon}\right\|_{X}+\varepsilon \gamma_{c}\left\|q_{N}-p^{\varepsilon}\right\|_{Y}\left\|q_{N}-p_{N}^{\varepsilon}\right\|_{Y} \\
& \quad+\gamma_{b}\left\|v_{N}-u_{N}^{\varepsilon}\right\|_{X}\left\|q_{N}-p^{\varepsilon}\right\|_{Y}+\gamma_{b}\left\|v_{N}-u^{\varepsilon}\right\|_{X}\left\|q_{N}-p_{N}^{\varepsilon}\right\|_{Y} .
\end{aligned}
$$

We now proceed as in the proof of Proposition 2.3.2: As the Galerkin projection (4.14) to (4.13) onto the low-dimensional reduced basis approximation spaces $X_{N}, Y_{N}$, the errors $e_{N}^{u, \varepsilon}$ and $e_{N}^{p, \varepsilon}$ satisfy

$$
a\left(e_{N}^{u, \varepsilon}, v_{N}\right)+b\left(v_{N}, e_{N}^{p, \varepsilon}\right)=0, \quad \forall v_{N} \in X_{N}
$$

Since $X_{N}, Y_{N}$ are stable, the inf-sup condition (2.17) and (4.26) provide that

$$
\begin{aligned}
\beta_{N}\left\|q_{N}-p_{N}^{\varepsilon}\right\|_{Y} & \leq \sup _{v_{N} \in X_{N}} \frac{b\left(v_{N}, q_{N}-p_{N}^{\varepsilon}\right)}{\left\|v_{N}\right\|_{X}}=\sup _{v_{N} \in X_{N}} \frac{b\left(v_{N}, q_{N}-p^{\varepsilon}\right)+b\left(v_{N}, e_{N}^{p, \varepsilon}\right)}{\left\|v_{N}\right\|_{X}} \\
& =\sup _{v_{N} \in X_{N}} \frac{b\left(v_{N}, q_{N}-p^{\varepsilon}\right)-a\left(e_{N}^{u, \varepsilon}, v_{N}\right)}{\left\|v_{N}\right\|_{X}} \\
& \leq \gamma_{b}\left\|q_{N}-p^{\varepsilon}\right\|_{Y}+\gamma_{a}\left\|e_{N}^{u, \varepsilon}\right\|_{X},
\end{aligned}
$$

where the last inequality follows from (2.8) and (2.9).

Combining (4.25) and (4.27) now yields

$$
\begin{aligned}
& \alpha_{a}\left\|v_{N}-u_{N}^{\varepsilon}\right\|_{X}^{2}+\varepsilon \alpha_{c}\left\|q_{N}-p_{N}^{\varepsilon}\right\|_{Y}^{2} \\
& \leq \gamma_{a}\left\|v_{N}-u^{\varepsilon}\right\|_{X}\left\|v_{N}-u_{N}^{\varepsilon}\right\|_{X}+\varepsilon \gamma_{c}\left\|q_{N}-p^{\varepsilon}\right\|_{Y}\left\|q_{N}-p_{N}^{\varepsilon}\right\|_{Y} \\
& \quad+\gamma_{b}\left\|v_{N}-u_{N}^{\varepsilon}\right\|_{X}\left\|q_{N}-p^{\varepsilon}\right\|_{Y}+\frac{\gamma_{b}}{\beta_{N}}\left\|v_{N}-u^{\varepsilon}\right\|_{X}\left(\gamma_{b}\left\|q_{N}-p^{\varepsilon}\right\|_{Y}+\gamma_{a}\left\|e_{N}^{u, \varepsilon}\right\|_{X}\right)
\end{aligned}
$$

from the triangle inequality $\left\|e_{N}^{u, \varepsilon}\right\|_{X} \leq\left\|u^{\varepsilon}-v_{N}\right\|_{X}+\left\|v_{N}-u_{N}^{\varepsilon}\right\|_{X}$, the above is further bounded by

$$
\begin{aligned}
\leq & {\left[\gamma_{a}\left(1+\frac{\gamma_{b}}{\beta_{N}}\right)\left\|v_{N}-u^{\varepsilon}\right\|_{X}+\gamma_{b}\left\|q_{N}-p^{\varepsilon}\right\|_{Y}\right]\left\|v_{N}-u_{N}^{\varepsilon}\right\|_{X} } \\
& +\varepsilon \gamma_{c}\left\|q_{N}-p^{\varepsilon}\right\|_{Y}\left\|q_{N}-p_{N}^{\varepsilon}\right\|_{Y}+\frac{\gamma_{b}^{2}}{\beta_{N}}\left\|v_{N}-u^{\varepsilon}\right\|_{X}\left\|q_{N}-p^{\varepsilon}\right\|_{Y}+\frac{\gamma_{a} \gamma_{b}}{\beta_{N}}\left\|v_{N}-u^{\varepsilon}\right\|_{X}^{2} .
\end{aligned}
$$

We apply Young's inequality with $\rho_{1}, \rho_{2}>0$ such that

$$
\begin{aligned}
& \left(2 \alpha_{a}-\rho_{1}\right)\left\|v_{N}-u_{N}^{\varepsilon}\right\|_{X}^{2}+\left(2 \varepsilon \alpha_{c}-\varepsilon^{2} \rho_{2}\right)\left\|q_{N}-p_{N}^{\varepsilon}\right\|_{Y}^{2} \\
& \leq \frac{1}{\rho_{1}}\left[\gamma_{a}\left(1+\frac{\gamma_{b}}{\beta_{N}}\right)\left\|v_{N}-u^{\varepsilon}\right\|_{X}+\gamma_{b}\left\|q_{N}-p^{\varepsilon}\right\|_{Y}\right]^{2}+\frac{\gamma_{c}^{2}}{\rho_{2}}\left\|q_{N}-p^{\varepsilon}\right\|_{Y}^{2} \\
& \quad+2 \frac{\gamma_{b}^{2}}{\beta_{N}}\left\|v_{N}-u^{\varepsilon}\right\|_{X}\left\|q_{N}-p^{\varepsilon}\right\|_{Y}+2 \frac{\gamma_{a} \gamma_{b}}{\beta_{N}}\left\|v_{N}-u^{\varepsilon}\right\|_{X}^{2}
\end{aligned}
$$


choosing $\rho_{1} \equiv \alpha_{a}$ and $\rho_{2} \equiv 2 \alpha_{c}$, we obtain $2 \varepsilon \alpha_{c}-\varepsilon^{2} \rho_{2}=2 \varepsilon(1-\varepsilon) \alpha_{c}>0$ for $\varepsilon \in(0,1)$ and therefore,

$$
\left\|v_{N}-u_{N}^{\varepsilon}\right\|_{X} \leq C\left(\left\|u^{\varepsilon}-v_{N}\right\|_{X}+\left\|p^{\varepsilon}-q_{N}\right\|_{Y}\right),
$$

where $C>0$ depends only on $\gamma_{a}, \alpha_{a}, \gamma_{c}, \alpha_{c}, \gamma_{b}$, and $\beta_{N}$. The statement thus follows from the triangle inequality, (4.27), and (4.28).

\subsection{A Posteriori Error Estimation}

Using the penalty formulation, we may now develop rigorous a posteriori error bounds that do not require the computation of lower bounds to inf-sup stability constants.

\subsubsection{Ingredients}

Before we are able to formulate our a posteriori error bounds, we again need to introduce two sets of ingredients. The first set of ingredients consists of lower (and upper bounds) to the truth coercivity constants (2.10) and (4.12),

$$
\begin{aligned}
& \alpha_{a}^{\mathrm{LB}}(\mu) \leq \alpha_{a}(\mu) \leq \alpha_{a}^{\mathrm{UB}}(\mu), \quad \forall \mu \in \mathcal{D} . \\
& \alpha_{c}^{\mathrm{LB}}(\mu) \leq \alpha_{c}(\mu) \leq \alpha_{c}^{\mathrm{UB}}(\mu), \quad
\end{aligned}
$$

The second set of ingredients consists of the dual norms of the residuals associated with the reduced basis approximation $\left(u_{N}^{\varepsilon}(\mu), p_{N}^{\varepsilon}(\mu)\right)$,

$$
\left\|r_{N}^{1, \varepsilon}(\cdot ; \mu)\right\|_{X^{\prime}}=\sup _{v \in X} \frac{r_{N}^{1, \varepsilon}(v ; \mu)}{\|v\|_{X}}, \quad\left\|r_{N}^{2, \varepsilon}(\cdot ; \mu)\right\|_{Y^{\prime}}=\sup _{q \in Y} \frac{r_{N}^{2, \varepsilon}(q ; \mu)}{\|q\|_{Y}}
$$

where, for all $\mu \in \mathcal{D}, r_{N}^{1, \varepsilon}(\cdot ; \mu) \in X^{\prime}$ and $r_{N}^{2, \varepsilon}(\cdot ; \mu) \in Y^{\prime}$ are defined as

$$
\begin{aligned}
& r_{N}^{1, \varepsilon}(v ; \mu) \equiv f(v ; \mu)-a\left(u_{N}^{\varepsilon}(\mu), v ; \mu\right)-b\left(v, p_{N}^{\varepsilon}(\mu) ; \mu\right), \quad \forall v \in X, \\
& r_{N}^{2, \varepsilon}(q ; \mu) \equiv g(q ; \mu)-b\left(u_{N}^{\varepsilon}(\mu), q ; \mu\right)+\varepsilon c\left(p_{N}^{\varepsilon}(\mu), q ; \mu\right), \quad \forall q \in Y \text {. }
\end{aligned}
$$

\subsubsection{Error Bound Formulation}

We can now state the following result.

Proposition 4.4.1. For $\varepsilon>0, \mu \in \mathcal{D}, N \in \mathbb{N}_{\max }$, and $\alpha_{a}^{\mathrm{LB}}(\mu), \alpha_{c}^{\mathrm{LB}}(\mu)$ satisfying (4.29), we define

$$
\Delta_{N}^{\varepsilon}(\mu) \equiv\left(\frac{\left\|r_{N}^{1, \varepsilon}(\cdot ; \mu)\right\|_{X^{\prime}}^{2}}{\alpha_{a}^{\mathrm{LB}}(\mu)}+\frac{\left\|r_{N}^{2, \varepsilon}(\cdot ; \mu)\right\|_{Y^{\prime}}^{2}}{\varepsilon \alpha_{c}^{\mathrm{LB}}(\mu)}\right)^{1 / 2} .
$$

Then, $\Delta_{N}^{\varepsilon}(\mu)$ is an upper bound for the error $e_{N}^{\varepsilon}(\mu)$ measured in the $\varepsilon$-dependent energy norm (4.19),

$$
\left\|e_{N}^{\varepsilon}(\mu)\right\|_{Z, \varepsilon, \mu} \leq \Delta_{N}^{\varepsilon}(\mu), \quad \forall \mu \in \mathcal{D}, \forall N \in \mathbb{N}_{\text {max }}
$$


Proof. We follow the proof of Proposition 4.1 in [35]. Let $\varepsilon>0, \mu \in \mathcal{D}$, and $N \in \mathbb{N}_{\text {max }}$. From (4.31), (4.32), and (4.13), the errors $e_{N}^{u, \varepsilon}(\mu)$ and $e_{N}^{p, \varepsilon}(\mu)$ satisfy the equations

$$
\begin{aligned}
a\left(e_{N}^{u, \varepsilon}(\mu), v ; \mu\right)+b\left(v, e_{N}^{p, \varepsilon}(\mu) ; \mu\right)=r_{N}^{1, \varepsilon}(v ; \mu), & \forall v \in X, \\
b\left(e_{N}^{u, \varepsilon}(\mu), q ; \mu\right)-\varepsilon c\left(e_{N}^{p, \varepsilon}(\mu), q ; \mu\right)=r_{N}^{2, \varepsilon}(q ; \mu), & \forall q \in Y .
\end{aligned}
$$

Setting $v=e_{N}^{u, \varepsilon}(\mu), q=e_{N}^{p, \varepsilon}(\mu)$ and subtracting the second from the first equation yields

$$
\begin{aligned}
\left\|e_{N}^{u, \varepsilon}(\mu)\right\|_{X, \mu}^{2}+\varepsilon\left\|e_{N}^{p, \varepsilon}(\mu)\right\|_{Y, \mu}^{2}=r_{N}^{1, \varepsilon}\left(e_{N}^{u, \varepsilon}(\mu) ; \mu\right)-r_{N}^{2, \varepsilon}\left(e_{N}^{p, \varepsilon}(\mu) ; \mu\right) & \\
& \leq\left\|r_{N}^{1, \varepsilon}(\cdot ; \mu)\right\|_{X^{\prime}}\left\|e_{N}^{u, \varepsilon}(\mu)\right\|_{X}+\left\|r_{N}^{2, \varepsilon}(\cdot ; \mu)\right\|_{Y^{\prime}}\left\|e_{N}^{p, \varepsilon}(\mu)\right\|_{Y}
\end{aligned}
$$

from (2.10) and (4.12), this is then further bounded by

$$
\begin{aligned}
& \leq \frac{\left\|r_{N}^{1, \varepsilon}(\cdot ; \mu)\right\|_{X^{\prime}}}{\sqrt{\alpha_{a}(\mu)}}\left\|e_{N}^{u, \varepsilon}(\mu)\right\|_{X, \mu}+\frac{\left\|r_{N}^{2, \varepsilon}(\cdot ; \mu)\right\|_{Y^{\prime}}}{\sqrt{\alpha_{c}(\mu)}}\left\|e_{N}^{p, \varepsilon}(\mu)\right\|_{Y, \mu} \\
& \leq\left(\left\|e_{N}^{u, \varepsilon}(\mu)\right\|_{X, \mu}^{2}+\varepsilon\left\|e_{N}^{p, \varepsilon}(\mu)\right\|_{Y, \mu}^{2}\right)^{1 / 2}\left(\frac{\left\|r_{N}^{1, \varepsilon}(\cdot ; \mu)\right\|_{X^{\prime}}^{2}}{\alpha_{a}(\mu)}+\frac{\left\|r_{N}^{2, \varepsilon}(\cdot ; \mu)\right\|_{Y^{\prime}}^{2}}{\varepsilon \alpha_{c}(\mu)}\right)^{1 / 2},
\end{aligned}
$$

where the second estimate is obtained from the Cauchy-Schwarz inequality for the Euclidean distance. The statement then directly follows from (4.29).

Again, as a measure of the quality of the proposed error estimators, we consider the effectivities

$$
\eta_{N}^{\varepsilon}(\mu) \equiv \frac{\Delta_{N}^{\varepsilon}(\mu)}{\left\|e_{N}^{\varepsilon}(\mu)\right\|_{Z, \varepsilon, \mu}}, \quad \varepsilon>0, \quad \mu \in \mathcal{D}, N \in \mathbb{N}_{\max } .
$$

We recall that effectivities $\geq 1$ indicate that the error estimate is rigorous; effectivities close to unity indicate that the error estimate is sharp. The following corollary specifies this behavior for the error bounds derived above.

Corollary 4.4.2. For $\varepsilon \in(0,1], \mu \in \mathcal{D}$, and $N \in \mathbb{N}_{\max }$, the effectivity $\eta_{N}^{\varepsilon}(\mu)$ associated with the error bound (4.33) satisfies

$$
1 \leq \eta_{N}^{\varepsilon}(\mu) \leq \frac{C_{3}(\mu)}{\sqrt{\varepsilon}}
$$

where the constant $C_{3}(\mu)>0$ may be expressed as

$$
C_{3}(\mu) \equiv\left(\frac{\left(\gamma_{b}(\mu)\right)^{2}}{\alpha_{a}^{\mathrm{LB}}(\mu) \alpha_{c}(\mu)}+\frac{\left(\gamma_{b}(\mu)\right)^{2}}{\alpha_{c}^{\mathrm{LB}}(\mu) \alpha_{a}(\mu)}+\frac{\left(\gamma_{a}(\mu)\right)^{2}}{\alpha_{a}^{\mathrm{LB}}(\mu) \alpha_{a}(\mu)}+\frac{\left(\gamma_{c}(\mu)\right)^{2}}{\alpha_{c}^{\mathrm{LB}}(\mu) \alpha_{c}(\mu)}\right)^{1 / 2} .
$$

Proof. Let $\varepsilon>0, \mu \in \mathcal{D}$, and $N \in \mathbb{N}_{\max }$. (Again, we suppress the argument $\mu$ for clarity of exposition.) The first inequality in (4.38) immediately follows from (4.34) in Proposition 4.4.1. In order to derive the upper bound for the effectivity, we note from (4.35) and (4.36) that the dual norms of the residuals satisfy

$$
\begin{aligned}
\left\|r_{N}^{1, \varepsilon}\right\|_{X^{\prime}} & \leq \gamma_{a}\left\|e_{N}^{u, \varepsilon}\right\|_{X}+\gamma_{b}\left\|e_{N}^{p, \varepsilon}\right\|_{Y} \leq \frac{\gamma_{a}}{\sqrt{\alpha_{a}}}\left\|e_{N}^{u, \varepsilon}\right\|_{X, \mu}+\frac{\gamma_{b}}{\sqrt{\alpha_{c}}}\left\|e_{N}^{p, \varepsilon}\right\|_{Y, \mu}, \\
\left\|r_{N}^{2, \varepsilon}\right\|_{Y^{\prime}} & \leq \gamma_{b}\left\|e_{N}^{u, \varepsilon}\right\|_{X}+\varepsilon \gamma_{c}\left\|e_{N}^{p, \varepsilon}\right\|_{Y} \leq \frac{\gamma_{b}}{\sqrt{\alpha_{a}}}\left\|e_{N}^{u, \varepsilon}\right\|_{X, \mu}+\varepsilon \frac{\gamma_{c}}{\sqrt{\alpha_{c}}}\left\|e_{N}^{p, \varepsilon}\right\|_{Y, \mu}
\end{aligned}
$$


by (2.8), (2.9), (2.10), (4.11), and (4.12). Using the Cauchy-Schwarz inequality for the Euclidean distance, we obtain

$$
\left\|r_{N}^{1, \varepsilon}\right\|_{X^{\prime}} \leq\left(\frac{\gamma_{a}^{2}}{\alpha_{a}}+\frac{\gamma_{b}^{2}}{\varepsilon \alpha_{c}}\right)^{1 / 2}\left\|e_{N}^{\varepsilon}\right\|_{Z, \varepsilon, \mu}, \quad\left\|r_{N}^{2, \varepsilon}\right\|_{Y^{\prime}} \leq\left(\frac{\gamma_{b}^{2}}{\alpha_{a}}+\varepsilon \frac{\gamma_{c}^{2}}{\alpha_{c}}\right)^{1 / 2}\left\|e_{N}^{\varepsilon}\right\|_{Z, \varepsilon, \mu} .
$$

Applying this to (4.33), we have

$$
\begin{aligned}
\left(\Delta_{N}^{\varepsilon}\right)^{2} & \leq\left[\frac{1}{\alpha_{a}^{\mathrm{LB}}}\left(\frac{\gamma_{a}^{2}}{\alpha_{a}}+\frac{\gamma_{b}^{2}}{\varepsilon \alpha_{c}}\right)+\frac{1}{\varepsilon \alpha_{c}^{\mathrm{LB}}}\left(\varepsilon \frac{\gamma_{c}^{2}}{\alpha_{c}}+\frac{\gamma_{b}^{2}}{\alpha_{a}}\right)\right]\left\|e_{N}^{\varepsilon}\right\|_{Z, \varepsilon, \mu}^{2} \\
& =\left[\frac{1}{\varepsilon}\left(\frac{\gamma_{b}^{2}}{\alpha_{a}^{\mathrm{LB}} \alpha_{c}}+\frac{\gamma_{b}^{2}}{\alpha_{c}^{\mathrm{LB}} \alpha_{a}}\right)+\frac{\gamma_{a}^{2}}{\alpha_{a}^{\mathrm{LB}} \alpha_{a}}+\frac{\gamma_{c}^{2}}{\alpha_{c}^{\mathrm{LB}} \alpha_{c}}\right]\left\|e_{N}^{\varepsilon}\right\|_{Z, \varepsilon, \mu}^{2}
\end{aligned}
$$

which finally yields the statement for $\varepsilon \in(0,1]$.

In the special case of a symmetric problem, the constant given in Corollary 4.4 .2 can be sharpened as follows.

Corollary 4.4.3. Let $a(\cdot, \cdot ; \mu)$ and $c(\cdot, \cdot ; \mu)$ be symmetric for all $\mu \in \mathcal{D}$. For $\varepsilon \in(0,1]$, $\mu \in \mathcal{D}$, and $N \in \mathbb{N}_{\max }$, the effectivity $\eta_{N}^{\varepsilon}(\mu)$ associated with the error bound (4.33) satisfies

$$
1 \leq \eta_{N}^{\varepsilon}(\mu) \leq \frac{C_{3}^{\mathrm{sym}}(\mu)}{\sqrt{\varepsilon}}
$$

where the constant $C_{3}^{\mathrm{sym}}(\mu)>0$ may be expressed as

$$
C_{3}^{\mathrm{sym}}(\mu) \equiv\left(\frac{\left(\gamma_{b}(\mu)\right)^{2}}{\alpha_{a}^{\mathrm{LB}}(\mu) \alpha_{c}(\mu)}+\frac{\left(\gamma_{b}(\mu)\right)^{2}}{\alpha_{c}^{\mathrm{LB}}(\mu) \alpha_{a}(\mu)}+\frac{\gamma_{a}(\mu)}{\alpha_{a}^{\mathrm{LB}}(\mu)}+\frac{\gamma_{c}(\mu)}{\alpha_{c}^{\mathrm{LB}}(\mu)}\right)^{1 / 2} \leq C_{3}(\mu) .
$$

Proof. Proceeding as in the previous proof, the dual norms of the residuals may now be estimated by

$$
\begin{aligned}
& \left\|r_{N}^{1, \varepsilon}\right\|_{X^{\prime}} \leq \sqrt{\gamma_{a}}\left\|e_{N}^{u, \varepsilon}\right\|_{X, \mu}+\gamma_{b}\left\|e_{N}^{p, \varepsilon}\right\|_{Y} \leq \sqrt{\gamma_{a}}\left\|e_{N}^{u, \varepsilon}\right\|_{X, \mu}+\frac{\gamma_{b}}{\sqrt{\alpha_{c}}}\left\|e_{N}^{p, \varepsilon}\right\|_{Y, \mu}, \\
& \left\|r_{N}^{2, \varepsilon}\right\|_{Y^{\prime}} \leq \gamma_{b}\left\|e_{N}^{u, \varepsilon}\right\|_{X}+\varepsilon \sqrt{\gamma_{c}}\left\|e_{N}^{p, \varepsilon}\right\|_{Y, \mu} \leq \frac{\gamma_{b}}{\sqrt{\alpha_{a}}}\left\|e_{N}^{u, \varepsilon}\right\|_{X, \mu}+\varepsilon \sqrt{\gamma_{c}}\left\|e_{N}^{p, \varepsilon}\right\|_{Y, \mu}
\end{aligned}
$$

from (4.35), (4.36), the Cauchy-Schwarz inequalities for the inner products $a(\cdot ; \cdot ; \mu)$ and $c(\cdot, \cdot ; \mu),(2.8),(2.9),(4.11)$, and $(2.10),(4.12)$. Therefore, (4.39) now becomes

$$
\left\|r_{N}^{1, \varepsilon}\right\|_{X^{\prime}} \leq\left(\gamma_{a}+\frac{\gamma_{b}^{2}}{\varepsilon \alpha_{c}}\right)^{1 / 2}\left\|e_{N}^{\varepsilon}\right\|_{Z, \varepsilon, \mu}, \quad\left\|r_{N}^{2, \varepsilon}\right\|_{Y^{\prime}} \leq\left(\frac{\gamma_{b}^{2}}{\alpha_{a}}+\varepsilon \gamma_{c}\right)^{1 / 2}\left\|e_{N}^{\varepsilon}\right\|_{Z, \varepsilon, \mu}
$$

and applying this to (4.33) again yields the desired result. Note that the constant $C_{3}^{\text {sym }}$ differs from $C_{3}$ in the last two terms; the inequality in (4.40) then follows as we clearly have $\frac{\gamma_{a}}{\alpha_{a}}, \frac{\gamma_{c}}{\alpha_{c}} \geq 1$ from the definitions in (2.8), (2.10) and (4.11), (4.12). 


\subsection{Construction of Reduced Basis Approximation Spaces}

We introduced several options to construct the low-dimensional reduced basis approximation space in $\S 2.5$. Accordingly, the reduced basis approximation space $Y_{N}$ for the Lagrange multiplier is defined as the span of several truth solutions $p^{\varepsilon}\left(\mu_{n}\right), 1 \leq n \leq N$, associated with chosen parameter snapshots $\mu_{1}, \ldots, \mu_{N} \in \mathcal{D}$ (see (2.69)),

$$
Y_{N} \equiv \operatorname{span}\left\{p^{\varepsilon}\left(\mu_{n}\right) \mid 1 \leq n \leq N\right\}=\operatorname{span}\left\{\xi_{n} \mid 1 \leq n \leq N\right\},
$$

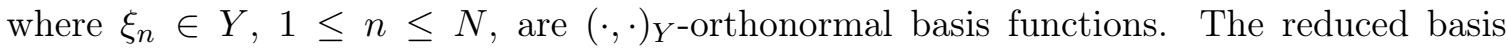
approximation space $X_{N}$ for the primal variable may then be constructed by using either Option 0, Option 1, Option 2, or Option 3; for the reader's convenience, we restate their definitions in Table 4.1 but refer to $\S 2.5$ for details on their respective properties. Again, for algebraic stability reasons, also $X_{N}$ is in particular expressed by orthonormal basis functions, i.e., $X_{N} \equiv \operatorname{span}\left\{\phi_{m} \mid 1 \leq m \leq N_{X}\right\}$, where $\phi_{m} \in X, 1 \leq m \leq N_{X}$, are $(\cdot, \cdot)_{X}$-orthonormal.

\begin{tabular}{c|c}
\hline Option & $X_{N}$ \\
\hline \hline 0 & $X_{N}^{0} \equiv \operatorname{span}\left\{u^{\varepsilon}\left(\mu_{n}\right) \mid 1 \leq n \leq N\right\}(\operatorname{see}(2.70))$ \\
\hline 1 & $X_{N}^{1} \equiv X_{N}^{0} \oplus \operatorname{span}\left\{T^{k} \xi_{n} \mid 1 \leq n \leq N, 1 \leq k \leq Q_{b}\right\}($ see $(2.77))$ \\
\hline 2 & $X_{N}^{2} \equiv X_{N}^{0} \oplus \operatorname{span}\left\{T_{\mu_{n}} \xi_{n} \mid 1 \leq n \leq N\right\}($ see $(2.78))$ \\
\hline 3 & $X_{N}^{3} \equiv X_{N}^{0} \oplus \operatorname{span}\left\{u^{\varepsilon}\left(\mu_{n}^{\prime}\right) \mid 1 \leq n \leq N\right\}($ see $(2.79))$ \\
\hline
\end{tabular}

Table 4.1.: Options to construct of the reduced basis approximation space $X_{N}$ for the primal variable, where the reduced basis approximation space $Y_{N}$ for the Lagrange multiplier is given as in (4.41).

We recall (see §4.3.2) that for all options considered in Table 4.1, the problem (4.14) is uniquely solvable and we obtain valid approximations $u_{N}^{\varepsilon}(\mu)$ and $p_{N}^{\varepsilon}(\mu)$ for $u^{\varepsilon}(\mu)$ and $p^{\varepsilon}(\mu)$, respectively. In contrast to the situation described in $\S 2.5$, we emphasize that also Option 0 no longer disqualifies itself a priori but may possibly provide useful results.

\subsection{Offline-Online Computational Procedure}

Again, we exploit the parameter-affine dependence (2.7) of the problem to invoke an efficient Offline-Online computational strategy.

\subsubsection{Reduced Basis Approximation}

Noting that for $\varepsilon>0$ and $\mu \in \mathcal{D}$, we can expand $u_{N}^{\varepsilon}(\mu) \in X_{N}$ and $p_{N}^{\varepsilon}(\mu) \in Y_{N}$ as

$$
u_{N}^{\varepsilon}(\mu)=\sum_{m=1}^{N_{X}} u_{N m}^{\varepsilon}(\mu) \phi_{m}, \quad p_{N}^{\varepsilon}(\mu)=\sum_{n=1}^{N_{Y}} p_{N n}^{\varepsilon}(\mu) \xi_{n}
$$

the reduced basis system (4.14) may be written as

$$
\sum_{m=1}^{N_{X}} \sum_{k=1}^{Q_{a}} \Theta_{a}^{k}(\mu) A_{N i m}^{k} u_{N m}^{\varepsilon}(\mu)+\sum_{n=1}^{N_{Y}} \sum_{k=1}^{Q_{b}} \Theta_{b}^{k}(\mu) B_{N n i}^{k} p_{N n}^{\varepsilon}(\mu)=\sum_{k=1}^{Q_{f}} \Theta_{f}^{k}(\mu) f_{N i}^{k}
$$


for all $1 \leq i \leq N_{X}$, and

$$
\sum_{m=1}^{N_{X}} \sum_{k=1}^{Q_{b}} \Theta_{b}^{k}(\mu) B_{N j m}^{k} u_{N m}^{\varepsilon}(\mu)-\varepsilon \sum_{n=1}^{N_{Y}} \sum_{k=1}^{Q_{c}} \Theta_{c}^{k}(\mu) C_{N j n}^{k} p_{N n}^{\varepsilon}(\mu)=\sum_{k=1}^{Q_{g}} \Theta_{g}^{k}(\mu) g_{N j}^{k},
$$

for all $1 \leq j \leq N_{Y}$; here, the $\mu$-independent quantities $A_{N i m}^{k}, B_{N n i}^{k}, f_{N i}^{k}$, and $g_{N j}^{k}$ are given as in $\S 2.6 .1$ and

$$
C_{N i j}^{k} \equiv c^{k}\left(\xi_{j}, \xi_{i}\right), \quad 1 \leq i, j \leq N_{Y}, 1 \leq k \leq Q_{c} .
$$

Again, the expensive Offline stage where we form and store all parameter-independent quantities enables the highly efficient Online evaluation of the reduced basis approximation for any parameter query; computational costs essentially remain the same as listed in §2.6.1.

\subsubsection{A Posteriori Error Bounds}

The computation of the a posteriori error bounds $\Delta_{N}^{\varepsilon}(\mu)$ again consists of two components: the calculation of the residual dual norms (4.30), and the calculation of the required lower (and upper) bounds (4.29) to the coercivity constants $\alpha_{a}(\mu)$ and $\alpha_{c}(\mu)$.

As in $\S 2.6 .2$, we use the $\mu$-affine expansions (2.7) and (4.42) to express the residual $r_{N}^{1, \varepsilon}(\cdot ; \mu)$ defined in (4.31) as

$$
\begin{aligned}
r_{N}^{1, \varepsilon}(v ; \mu)=\sum_{k=1}^{Q_{f}} \Theta_{f}^{k}(\mu) f^{k}(v) & -\sum_{m=1}^{N_{X}} \sum_{k=1}^{Q_{a}} u_{N m}^{\varepsilon}(\mu) \Theta_{a}^{k}(\mu) a^{k}\left(\phi_{m}, v\right) \\
& -\sum_{n=1}^{N_{Y}} \sum_{k=1}^{Q_{b}} p_{N n}^{\varepsilon}(\mu) \Theta_{b}^{k}(\mu) b^{k}\left(v, \xi_{n}\right), \quad \forall v \in X,
\end{aligned}
$$

and the residual $r_{N}^{2, \varepsilon}(\cdot ; \mu)$ defined in $(4.32)$ as

$$
\begin{aligned}
r_{N}^{2, \varepsilon}(q ; \mu)=\sum_{k=1}^{Q_{g}} \Theta_{g}^{k}(\mu) g^{k}(q) & -\sum_{m=1}^{N_{X}} \sum_{k=1}^{Q_{b}} u_{N m}^{\varepsilon}(\mu) \Theta_{b}^{k}(\mu) b^{k}\left(\phi_{m}, q\right) \\
& +\varepsilon \sum_{n=1}^{N_{Y}} \sum_{k=1}^{Q_{c}} p_{N n}^{\varepsilon}(\mu) \Theta_{c}^{k}(\mu) c^{k}\left(\xi_{n}, q\right), \quad \forall q \in Y .
\end{aligned}
$$

Setting $Q_{1} \equiv Q_{f}+N_{X} Q_{a}+N_{Y} Q_{b}$ and $Q_{2}^{\varepsilon} \equiv Q_{g}+N_{X} Q_{b}+N_{Y} Q_{c}$, this can be written succinctly as

$$
\begin{aligned}
& r_{N}^{1, \varepsilon}(v ; \mu)=\sum_{k=1}^{Q_{1}} \Theta_{N}^{1, \varepsilon, k}(\mu) r_{N}^{1, \varepsilon, k}(v), \quad \forall v \in X, \\
& r_{N}^{2, \varepsilon}(q ; \mu)=\sum_{k=1}^{Q_{2}^{\varepsilon}} \Theta_{N}^{2, \varepsilon, k}(\mu) r_{N}^{2, \varepsilon, k}(q), \quad \forall q \in Y,
\end{aligned}
$$

where the coefficient functions $\Theta_{N}^{1, \varepsilon, k}(\mu), 1 \leq k \leq Q_{1}$, and $\Theta_{N}^{2, \varepsilon, k}(\mu), 1 \leq k \leq Q_{2}^{\varepsilon}$, depend on $\mu$ explicitly through the coefficient functions $\Theta_{\mathcal{I}}^{k}(\mu), 1 \leq k \leq Q_{\mathcal{I}}, \mathcal{I}=a, b, c, f, g$, but 
also implicitly through $u_{N}^{\varepsilon}(\mu)$ and $p_{N}^{\varepsilon}(\mu) ; r_{N}^{1, \varepsilon, k}(\cdot) \in X^{\prime}, 1 \leq k \leq Q_{1}$, and $r_{N}^{2, \varepsilon, k}(\cdot) \in Y^{\prime}$, $1 \leq k \leq Q_{2}^{\varepsilon}$, are parameter-independent linear functionals.

We denote by $\hat{e}_{N}^{1, \varepsilon, k} \in X$ and $\hat{e}_{N}^{2, \varepsilon, k} \in Y$ the Riesz representers associated with $r_{N}^{1, \varepsilon, k}(\cdot)$ and $r_{N}^{2, \varepsilon, k}(\cdot)$, respectively, which are obtained as the solutions to the $\mu$-independent linear problems

$$
\begin{aligned}
& \left(\hat{e}_{N}^{1, \varepsilon, k}, v\right)_{X}=r_{N}^{1, \varepsilon, k}(v), \quad \forall v \in X, \quad \forall 1 \leq k \leq Q_{1}, \\
& \left(\hat{e}_{N}^{2, \varepsilon, k}, q\right)_{Y}=r_{N}^{2, \varepsilon, k}(q), \quad \forall q \in Y, \quad \forall 1 \leq k \leq Q_{2}^{\varepsilon} .
\end{aligned}
$$

By (4.43) and (4.44), the Riesz representers $\hat{e}_{N}^{1, \varepsilon}(\mu) \in X$ and $\hat{e}_{N}^{2, \varepsilon}(\mu) \in Y$ associated with the residuals $r_{N}^{1, \varepsilon}(\cdot ; \mu)$ and $r_{N}^{2, \varepsilon}(\cdot ; \mu)$ are then given by

$$
\hat{e}_{N}^{1, \varepsilon}(\mu)=\sum_{k=1}^{Q_{1}} \Theta_{N}^{1, \varepsilon, k}(\mu) \hat{e}_{N}^{1, \varepsilon, k}, \quad \hat{e}_{N}^{2, \varepsilon}(\mu)=\sum_{k=1}^{Q_{2}^{\varepsilon}} \Theta_{N}^{2, \varepsilon, k}(\mu) \hat{e}_{N}^{2, \varepsilon, k},
$$

and it finally follows that

$$
\begin{gathered}
\left\|r_{N}^{1, \varepsilon}(\cdot ; \mu)\right\|_{X^{\prime}}^{2}=\left(\hat{e}_{N}^{1, \varepsilon}(\mu), \hat{e}_{N}^{1, \varepsilon}(\mu)\right)_{X}=\sum_{k, l=1}^{Q_{1}} \Theta_{N}^{1, \varepsilon, k}(\mu) \Theta_{N}^{1, \varepsilon, l}(\mu)\left(\hat{e}_{N}^{1, \varepsilon, k}, \hat{e}_{N}^{1, \varepsilon, l}\right)_{X} \\
\left\|r_{N}^{2, \varepsilon}(\cdot ; \mu)\right\|_{Y^{\prime}}^{2}=\left(\hat{e}_{N}^{2, \varepsilon}(\mu), \hat{e}_{N}^{2, \varepsilon}(\mu)\right)_{Y}=\sum_{k, l=1}^{Q_{2}^{\varepsilon}} \Theta_{N}^{2, \varepsilon, k}(\mu) \Theta_{N}^{2, \varepsilon, l}(\mu)\left(\hat{e}_{N}^{2, \varepsilon, k}, \hat{e}_{N}^{2, \varepsilon, l}\right)_{Y}
\end{gathered}
$$

We may now summarize the Offline-Online decomposition.

In the Offline stage, we find the Riesz representers $\hat{e}_{N}^{1, \varepsilon, k}, 1 \leq k \leq Q_{1}$, and $\hat{e}_{N}^{2, \varepsilon, k}, 1 \leq k \leq$ $Q_{2}^{\varepsilon}$, and form the associated inner products $\left(\hat{e}_{N}^{1, \varepsilon, k}, \hat{e}_{N}^{1, \varepsilon, l}\right)_{X}, 1 \leq k, l \leq Q_{1}$, and $\left(\hat{e}_{N}^{2, \varepsilon, k}, \hat{e}_{N}^{2, \varepsilon, l}\right)_{Y}$, $1 \leq k, l \leq Q_{2}^{\varepsilon}$. Again (see $\$ 2.6 .2$ ), these computations clearly depend on $\mathcal{N}$ : We have to solve $Q_{1}$ and $Q_{2}^{\varepsilon}$ linear problems of type (4.45) and (4.46), respectively, and to compute $\left(Q_{1}\right)^{2}$ and $\left(Q_{2}^{\varepsilon}\right)^{2}$ inner products in $X$ and $Y$. In the Online stage, given the reduced basis coefficients $u_{N m}^{\varepsilon}(\mu), 1 \leq m \leq N_{X}$, and $p_{N n}^{\varepsilon}(\mu), 1 \leq n \leq N_{Y}$, we then compute the coefficient functions $\Theta_{N}^{1, \varepsilon, k}(\mu), 1 \leq k \leq Q_{1}$, and $\Theta_{N}^{2, \varepsilon, k}(\mu), 1 \leq k \leq Q_{2}^{\varepsilon}$, and assemble the sums (4.47) and (4.48) at $O\left(\left(Q_{1}\right)^{2}\right)=O\left(\left(Q_{f}+N_{X} Q_{a}+N_{Y} Q_{b}\right)^{2}\right)$ and $O\left(\left(Q_{2}^{\varepsilon}\right)^{2}\right)=$ $O\left(\left(Q_{g}+N_{X} Q_{b}+N_{Y} Q_{c}\right)^{2}\right)$ operations, respectively. As desired, the operation count for the Online stage is thus independent of $\mathcal{N}$.

We obtain (Online-)efficient lower and upper bounds (4.29) to the coercivity constants $\alpha_{a}(\mu)$ and $\alpha_{c}(\mu)$ by the SCM methodology described in $\S 2.6 .2$.

\subsection{Adaptive Sampling Procedures}

We discussed adaptive sampling procedures constructing reduced basis approximation spaces $\left(X_{N}, Y_{N}\right), N \in \mathbb{N}_{\max }$, that are computationally efficient in $\S 2.7$. Again, we consider the standard greedy sampling process combined with Option 2 (see §2.7, Algorithm 1) and the sampling procedures Algorithm 2 and Algorithm 3 where the need for stabilization is recognized adaptively. In addition, according to our observations in $\S 4.5$, a standard greedy 
approach (see §2.7) based on Option 0 (see Table 4.1) cannot categorically be excluded from further considerations but may provide useful results; the exact sampling procedure is therefore summarized in Algorithm 4. In contrast to the other sampling processes, we here do not pursue additional stabilization techniques but construct $X_{N}$ and $Y_{N}$ based solely on the respective truth solutions associated with the greedily selected parameter snapshots. However, we bear in mind that for penalty parameters $\varepsilon$ tending to zero, a priori error estimates in $\S 4.3 .3$ suggest poor convergence for approximation spaces $X_{N}, Y_{N}$ that exhibit small inf-sup constants $\beta_{N}(\mu)$ (see (2.17)).

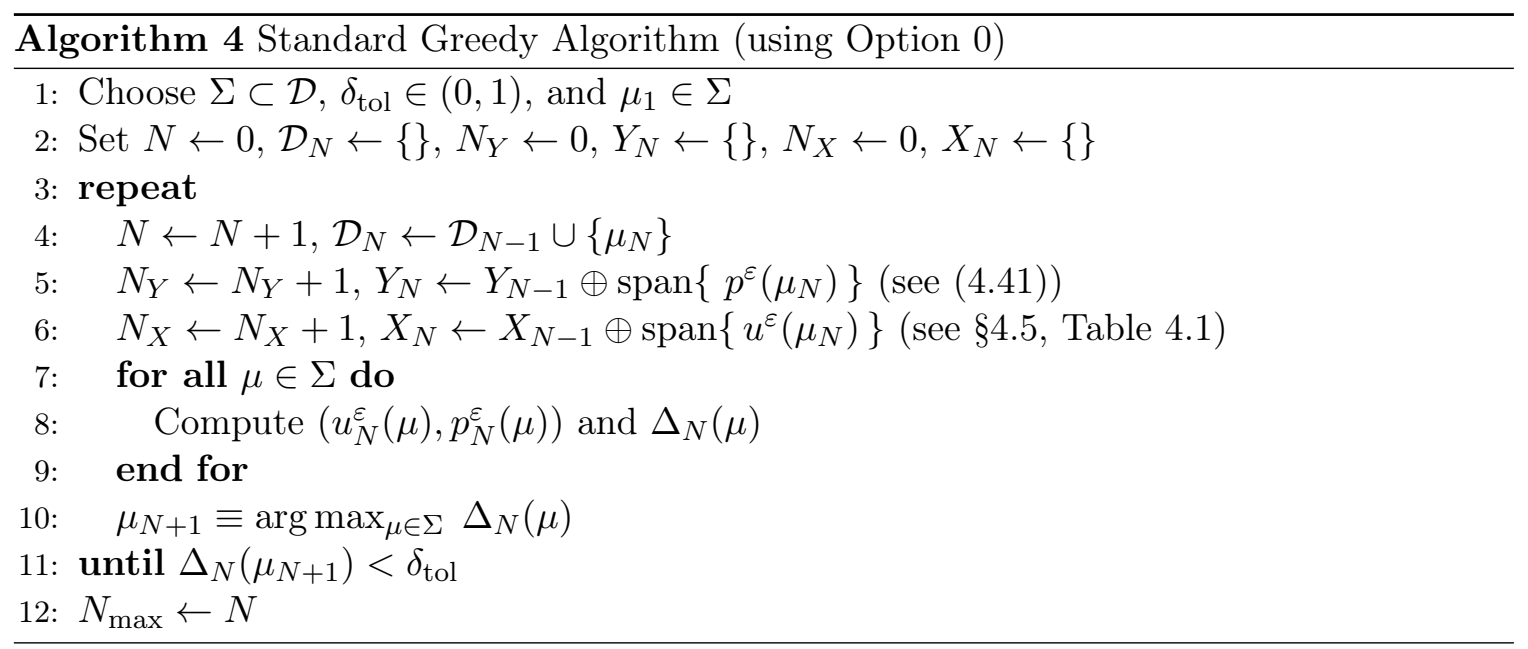

Remark 4.7.1. We here note that Algorithm 2 and Algorithm 3 involve the (Online-)efficient estimation of the truth Brezzi inf-sup constants $\beta_{\mathrm{Br}}(\mu)$ and are therefore not optimally suited to the penalty context where we aim to avoid such computations. However, the estimated truth Brezzi inf-sup constants are only used as reference values for the reduced basis inf-sup constants $\beta_{N}(\mu)$ (see (2.101)). In the penalty context, such a reference could for example also be given by $\beta_{\mathrm{Br}}\left(\mu_{\text {ref }}\right)$ for a chosen reference parameter $\mu_{\text {ref }} \in \mathcal{D}$.

\subsection{Concluding Remarks}

We present in this chapter the abstract framework of a new reduced basis method for saddle point problems based on a penalty formulation. At the expense of a less accurate truth approximation, the proposed approach enables the circumvention of the inf-sup condition. The analysis demonstrates how this affects the construction of reduced basis approximation spaces as well as the development of a posteriori error bounds.

Through the introduction of the penalty term, fulfillment of the inf-sup condition (2.17) is no longer a compulsory requirement for the reduced basis approximation spaces. In case of small inf-sup constants, a priori error estimates predict poor convergence for penalty parameters tending to zero; however, computationally efficient construction techniques where we do not enrich the reduced basis approximation space for the primal variable cannot categorically be excluded from further considerations but may possibly provide useful results. 
The developed rigorous a posteriori error bounds are independent of inf-sup stability constants and can therefore be computed very easily. However, they do depend on the penalty parameter $\varepsilon$ : As $\varepsilon$ decreases and we approach the nonpenalized problem, the error bounds become increasingly pessimistic. Nevertheless, with associated effectivities growing by an order of $O\left(\frac{1}{\sqrt{\varepsilon}}\right)$, these effects are relatively benign. 



\section{Chapter 5}

\section{Approximation of the Stokes Equations by Regularization or Penalty}

\subsection{Introduction}

First introduced in the context of constrained optimization, the penalty method gained popularity as a numerical solution method for the Stokes and Navier-Stokes equations in the 1980s and 1990s (see, for example, $[9,20,52,81,82]$ and the review papers $[51,91]$ ). Combined with reduced integration techniques [112], it allows the elimination of the pressure and circumvention of the Babuška-Brezzi inf-sup condition [9, 13].

In the previous chapter, we presented an abstract framework of how the penalty formulation may be employed in the reduced basis context. With the increase in speed and memory capacity of computing devices, the penalty approach lost popularity in the finite element community after the 1990s; in the parametric reduced basis setting, it still provides a reduction of computational effort that is significant. In this chapter, we apply the method developed in Chapter 4 to a perturbed Stokes flow in a parametrized domain. Considering the model problem introduced in $\S 3.2$, numerical results demonstrate the effects of the penalty term in practice.

The chapter is organized as follows: In $\S 5.2$, we derive the penalty formulation for the Stokes model problem introduced in $§ 3.2$. In $\S 5.3$, we then apply the reduced basis approach discussed in $\S 4$ : Numerical results illustrate the effects of the penalty term on (i) the perturbed truth velocity and pressure approximations, (ii) convergence of the reduced basis approximations, (iii) computational efficiency, and (iv) error bound effectivity.

\subsection{Model Problem}

We again consider a Stokes flow through a two-dimensional microchannel with a parametrized rectangular obstacle as described in $\S 3.2$. We now introduce a penalty term to the continuity equation (3.2),

$$
\nabla \cdot \tilde{u}_{\mathrm{e}, \text { inh }}^{\varepsilon}=-\varepsilon \tilde{p}_{\mathrm{e}}^{\varepsilon} \quad \text { in } \tilde{\Omega},
$$

for some small penalty parameter $\varepsilon>0$. We recall that (5.1) essentially approximates the fluid as nearly incompressible. From (3.1), (5.1), and (3.3), the governing equations for the 
velocity $\tilde{u}_{\mathrm{e}, \mathrm{inh}}^{\varepsilon}$ and the pressure $\tilde{p}_{\mathrm{e}}^{\varepsilon}$ are then

$$
\begin{aligned}
-\tilde{\Delta} \tilde{u}_{\mathrm{e}, \mathrm{inh}}^{\varepsilon}+\tilde{\nabla} \tilde{p}_{\mathrm{e}}^{\varepsilon}=0 & \text { in } \tilde{\Omega}, \\
\tilde{\nabla} \cdot \tilde{u}_{\mathrm{e}, \mathrm{inh}}^{\varepsilon}+\varepsilon \tilde{p}_{\mathrm{e}}^{\varepsilon}=0 & \text { in } \tilde{\Omega},
\end{aligned}
$$

with boundary conditions

$$
\tilde{u}_{\mathrm{e}, \text { inh }}^{\varepsilon}=h \quad \text { on } \Gamma_{\mathrm{in}}, \quad \tilde{u}_{\mathrm{e}, \text { inh }}^{\varepsilon}=0 \quad \text { on } \tilde{\Gamma}_{0}, \quad \frac{\partial \tilde{u}_{\mathrm{e}, \text { inh }}^{\varepsilon}}{\partial \tilde{n}}=\tilde{p}_{\mathrm{e}}^{\varepsilon} \tilde{n} \quad \text { on } \tilde{\Gamma}_{\text {out }} ;
$$

here, $\tilde{\Delta}$ and $\tilde{\nabla}$ again denote the Laplacian and gradient operator over the physical domain $\tilde{\Omega}$ (see Appendix A), respectively, $\tilde{n}$ is the unit outward normal, and $h: \mathbb{R}^{2} \rightarrow \mathbb{R}^{2}$ is given by $h(x)=h\left(x_{1}, x_{2}\right) \equiv\left(4 x_{2}\left(1-x_{2}\right), 0\right)$ for all $x=\left(x_{1}, x_{2}\right) \in \mathbb{R}^{2}$.

Following the steps discussed in $\S 3.2 .2$ and $\S 3.2 .3$, the problem (5.2)-(5.4) may be written as a parametrized saddle point problem of the form (4.3). Here, for any $\mu \in \mathcal{D}$, the bilinear forms $a(\cdot, \cdot ; \mu), b(\cdot, \cdot ; \mu)$ and the linear functionals $f(\cdot ; \mu), g(\cdot ; \mu)$ are given as in (3.8)-(3.11); accordingly, the bilinear form $c(\cdot, \cdot ; \mu): Y_{\mathrm{e}} \times Y_{\mathrm{e}} \rightarrow \mathbb{R}$ represents the $L^{2}$-inner product over the physical domain $\tilde{\Omega}$ formulated on the reference domain $\Omega$ (see §3.2.3),

$$
c(p, q ; \mu)=\sum_{s=1}^{S} \frac{1}{\left|\operatorname{det}\left(A^{s}(\mu)\right)\right|} \int_{\Omega^{s}} p q d x, \quad \forall p, q \in Y_{\mathrm{e}} .
$$

The bilinear forms $a(\cdot, \cdot ; \mu), b(\cdot, \cdot ; \mu)$, and $c(\cdot, \cdot ; \mu)$ then satisfy the assumptions (2.1)-(2.4), (4.1), and (4.2), and we thus find ourselves in the setting introduced in $\S 4.2 .1$. We particularly have $Q_{a}=10, Q_{b}=6, Q_{f}=Q_{g}=1$, and $Q_{c}=5$ in the respective $\mu$-affine expansions (2.7). Choosing again the truth approximation spaces $X$ and $Y$ as the standard conforming $\mathbb{P}_{2}-\mathbb{P}_{1}$ Taylor-Hood finite element approximation subspaces [100] over the regular triangulation $\mathcal{T}_{\Omega}$, we ensure that also (2.11) is satisfied and therefore recover the situation described in $\S 4.2 .2$.

\subsection{Numerical Results}

We now apply the reduced basis methodology developed in $\S 4.3-\S 4.7$ to our model problem. In this section, all numerical results are attained using the open source software rb00mit [65], an implementation of the reduced basis framework within the $\mathrm{C}++$ parallel finite element library libMesh [62].

\subsubsection{Truth Approximation}

First, we compare our perturbed truth approximations $u^{\varepsilon}(\mu)$ and $p^{\varepsilon}(\mu)$ with the solutions $u(\mu)$ and $p(\mu)$ of the nonpenalized Stokes equations $(\varepsilon=0)$.

Again based on a fine mesh with 16,602 elements, the truth system (4.13) has a dimension of $\mathcal{N}=72,076$. Figure 5.1 shows the velocity streamlines computed using $\varepsilon=$ $10^{-2}, 10^{-3}, 10^{-4}$, and $10^{-5}$; for comparison, they are also plotted for $\varepsilon=0$. We observe that our penalized truth approximation is certainly less-than-perfect, but for most engineering applications, the approximation is sufficiently accurate even for reasonably large values 
(a) $\varepsilon=10^{-2}$

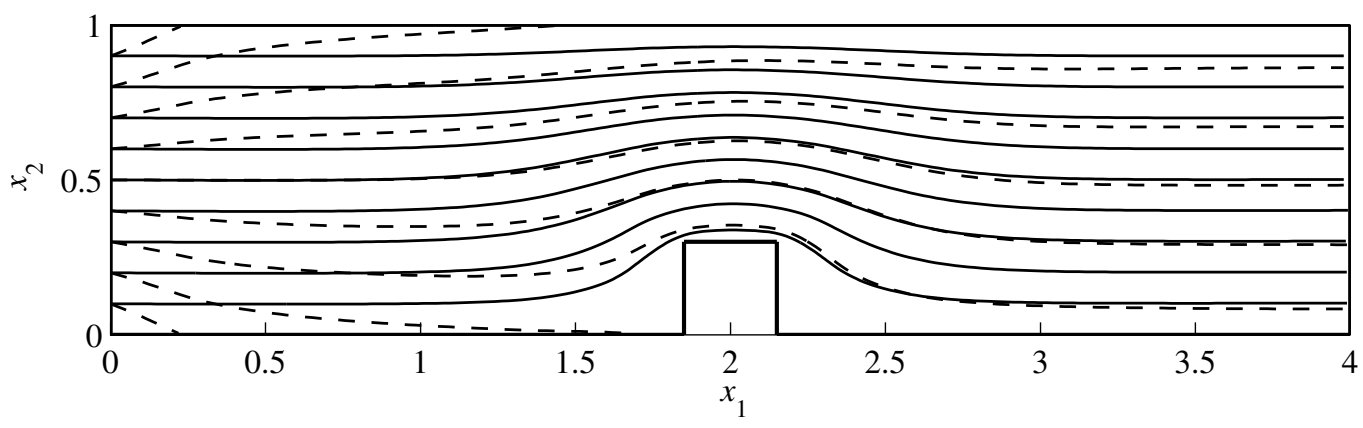

(b) $\varepsilon=10^{-3}$

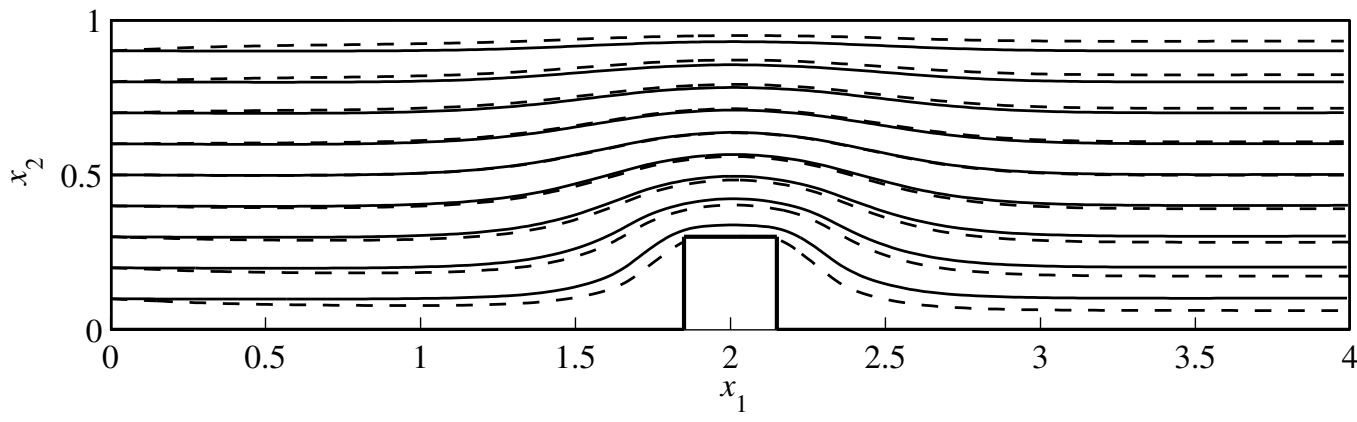

(c) $\varepsilon=10^{-4}$

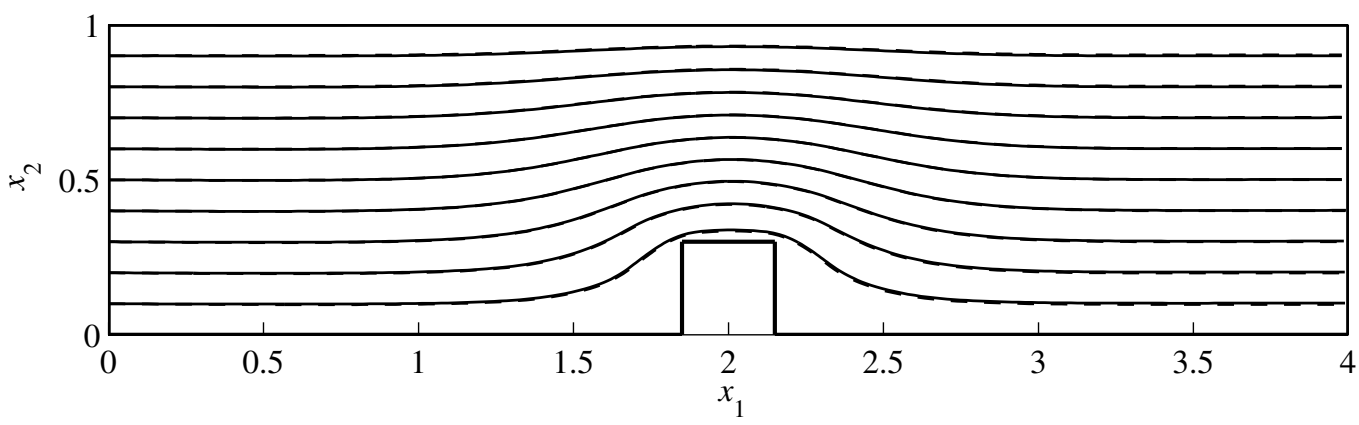

(d) $\varepsilon=10^{-5}$

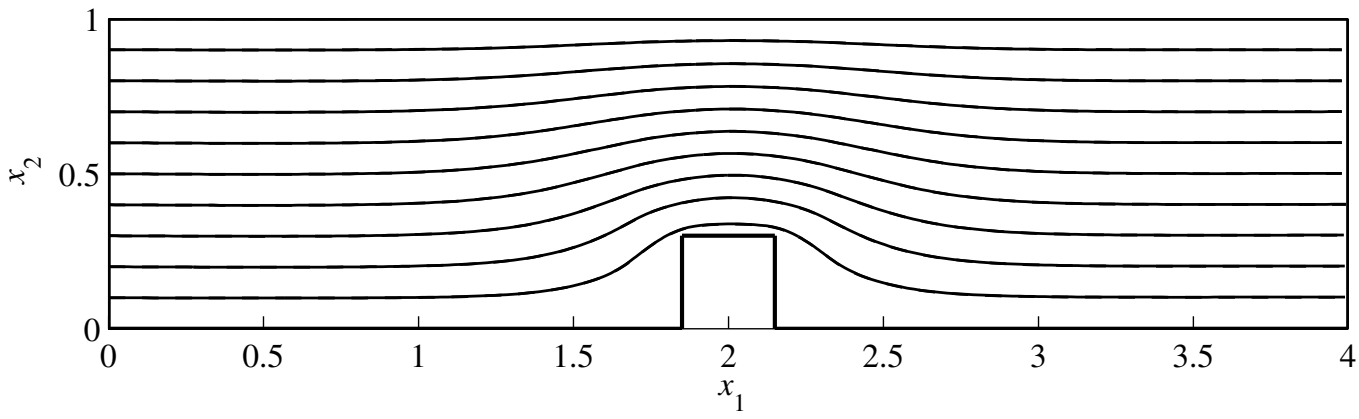

Figure 5.1.: Velocity streamlines computed using (a) $\varepsilon=10^{-2}$, (b) $\varepsilon=10^{-3}$, (c) $\varepsilon=10^{-4}$, and (d) $\varepsilon=10^{-5}$; comparison with the solution for $\varepsilon=0$ (solid). 


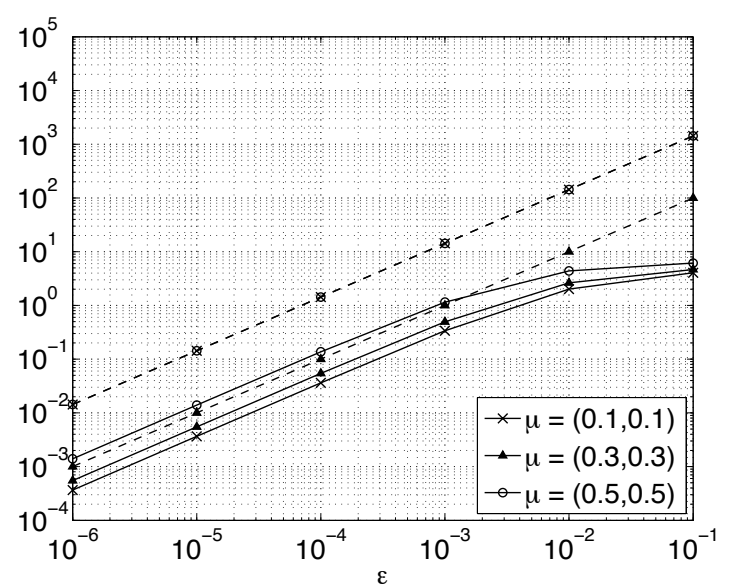

(a)

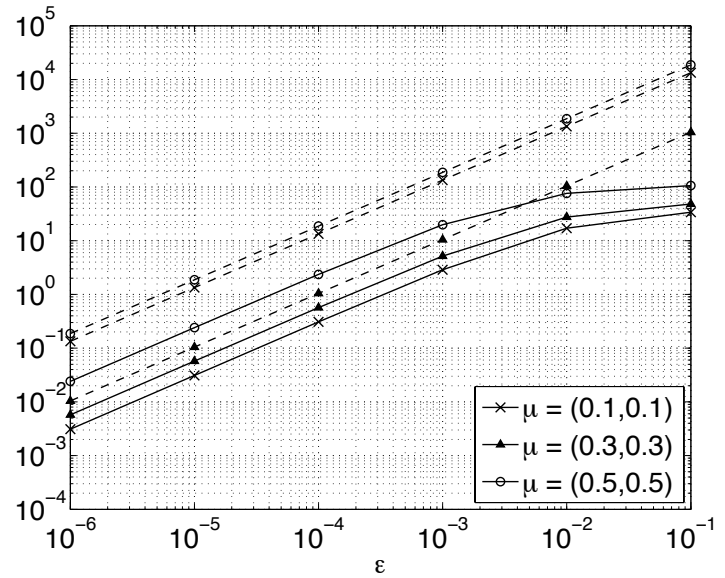

(b)

Figure 5.2.: Errors in the penalized truth approximations and associated upper bounds (see Proposition 4.2.4) as functions of the penalty parameter $\varepsilon$ for different parameter values $\mu$ : (a) $\left\|u(\mu)-u^{\varepsilon}(\mu)\right\|_{X}$ (solid) and $\left(C_{2}^{\text {sym }}(\mu) / \sqrt{\alpha_{a}(\mu)}\right) \varepsilon$ (dashed), (b) $\left\|p(\mu)-p^{\varepsilon}(\mu)\right\|_{Y}$ (solid) and $\left(\sqrt{\gamma_{a}(\mu)} / \beta_{\mathrm{Br}}(\mu)\right) C_{2}^{\mathrm{sym}}(\mu) \varepsilon$ (dashed).

of the penalty parameter $\varepsilon$. This is further illustrated in Fig. 5.2, which presents the error introduced by the penalty term as a function of $\varepsilon$. Moreover, the results in Fig. 5.2 clearly manifest the $O(\varepsilon)$-dependence of the error predicted by Proposition 4.2.3 and Proposition 4.2.4.

\subsubsection{Reduced Basis Approximation Spaces}

We now turn to the reduced basis approximation. We here present numerical results for the different options to construct the reduced basis approximation spaces $X_{N}$ and $Y_{N}$. As discussed in $\S 4.5$ and $\S 4.7$, we now build $X_{N}$ and $Y_{N}$ by using either Algorithm 1, Algorithm 2, Algorithm 3, or Algorithm 4. All sampling procedures are based on an exhaustive random sample $\Sigma \subset \mathcal{D}$ of size $|\Sigma|=4,900$ and the relative error bound $\Delta_{N}(\mu) \equiv \Delta_{N}^{\varepsilon}(\mu) /\left\|e_{N}^{\varepsilon}(\mu)\right\|_{Z, \varepsilon, \mu}$ (see (4.33)); in Algorithm 2 and Algorithm 3, we further set $\delta_{\text {tol }}^{\beta}=0.1$ (see $\S 2.7$ ).

We first compare the resulting reduced basis approximation spaces $X_{N}, Y_{N}$ with respect to stability. We recall that in Algorithm 1, Algorithm 2, and Algorithm 3, the spaces are stabilized through an additional enrichment of the reduced basis approximation space $X_{N}$ for the primal variable; in Algorithm 4, we abandon any special stabilization techniques. Table 5.1 now provides minimum values of the inf-sup constants $\beta_{N}(\mu)$ (see $(2.17)$ ) for different values of the penalty parameter $\varepsilon$. In case of Algorithm 1, Algorithm 2, and Algorithm 3, the inf-sup constants $\beta_{N}(\mu)$ are essentially constant in $N$ with values of approximately $8 \cdot 10^{-2}$ (see Table 5.1(a), (b), and (c)); using Algorithm 4, we obtain much lower values that range from $1.5 \cdot 10^{-2}$ to $7 \cdot 10^{-5}$ (see Table 5.1(d)). Furthermore, we note that the constants $\beta_{N}(\mu)$ show in neither case a noticeable dependence on the penalty parameter $\varepsilon$. 
(a) Algorithm 1

\begin{tabular}{c|c|c|c|c|c}
\hline$\varepsilon$ & $\beta_{5}(\mu)$ & $\beta_{10}(\mu)$ & $\beta_{20}(\mu)$ & $\beta_{30}(\mu)$ & $\beta_{40}(\mu)$ \\
\hline $10^{-2}$ & $8.574 \cdot 10^{-2}$ & $8.529 \cdot 10^{-2}$ & $8.471 \cdot 10^{-2}$ & $8.370 \cdot 10^{-2}$ & $8.283 \cdot 10^{-2}$ \\
$10^{-3}$ & $7.894 \cdot 10^{-2}$ & $7.899 \cdot 10^{-2}$ & $7.897 \cdot 10^{-2}$ & $7.901 \cdot 10^{-2}$ & $7.899 \cdot 10^{-2}$ \\
$10^{-4}$ & $7.850 \cdot 10^{-2}$ & $7.853 \cdot 10^{-2}$ & $7.848 \cdot 10^{-2}$ & $7.856 \cdot 10^{-2}$ & $7.860 \cdot 10^{-2}$ \\
$10^{-5}$ & $7.846 \cdot 10^{-2}$ & $7.848 \cdot 10^{-2}$ & $7.844 \cdot 10^{-2}$ & $7.851 \cdot 10^{-2}$ & $7.855 \cdot 10^{-2}$ \\
\hline
\end{tabular}

(b) Algorithm 2

\begin{tabular}{c|c|c|c|c|c}
\hline$\varepsilon$ & $\beta_{5}(\mu)$ & $\beta_{10}(\mu)$ & $\beta_{20}(\mu)$ & $\beta_{30}(\mu)$ & $\beta_{40}(\mu)$ \\
\hline $10^{-2}$ & $7.846 \cdot 10^{-2}$ & $8.061 \cdot 10^{-2}$ & $7.941 \cdot 10^{-2}$ & $7.848 \cdot 10^{-2}$ & $7.732 \cdot 10^{-2}$ \\
$10^{-3}$ & $7.781 \cdot 10^{-2}$ & $7.772 \cdot 10^{-2}$ & $7.773 \cdot 10^{-2}$ & $7.705 \cdot 10^{-2}$ & $7.771 \cdot 10^{-2}$ \\
$10^{-4}$ & $7.757 \cdot 10^{-2}$ & $7.747 \cdot 10^{-2}$ & $7.738 \cdot 10^{-2}$ & $7.755 \cdot 10^{-2}$ & $7.764 \cdot 10^{-2}$ \\
$10^{-5}$ & $7.754 \cdot 10^{-2}$ & $7.745 \cdot 10^{-2}$ & $7.735 \cdot 10^{-2}$ & $7.752 \cdot 10^{-2}$ & $7.761 \cdot 10^{-2}$ \\
\hline
\end{tabular}

(c) Algorithm 3

\begin{tabular}{c|c|c|c|c|c}
\hline$\varepsilon$ & $\beta_{5}(\mu)$ & $\beta_{10}(\mu)$ & $\beta_{20}(\mu)$ & $\beta_{30}(\mu)$ & $\beta_{40}(\mu)$ \\
\hline $10^{-2}$ & $8.089 \cdot 10^{-2}$ & $8.059 \cdot 10^{-2}$ & $7.960 \cdot 10^{-2}$ & $7.801 \cdot 10^{-2}$ & $7.786 \cdot 10^{-2}$ \\
$10^{-3}$ & $7.610 \cdot 10^{-2}$ & $7.793 \cdot 10^{-2}$ & $7.715 \cdot 10^{-2}$ & $7.688 \cdot 10^{-2}$ & $7.606 \cdot 10^{-2}$ \\
$10^{-4}$ & $7.656 \cdot 10^{-2}$ & $7.761 \cdot 10^{-2}$ & $7.699 \cdot 10^{-2}$ & $7.723 \cdot 10^{-2}$ & $7.644 \cdot 10^{-2}$ \\
$10^{-5}$ & $7.682 \cdot 10^{-2}$ & $7.767 \cdot 10^{-2}$ & $7.742 \cdot 10^{-2}$ & $7.739 \cdot 10^{-2}$ & $7.725 \cdot 10^{-2}$ \\
\hline
\end{tabular}

(d) Algorithm 4

\begin{tabular}{c|c|c|c|c|c}
\hline$\varepsilon$ & $\beta_{5}(\mu)$ & $\beta_{10}(\mu)$ & $\beta_{20}(\mu)$ & $\beta_{30}(\mu)$ & $\beta_{40}(\mu)$ \\
\hline $10^{-2}$ & $2.605 \cdot 10^{-3}$ & $2.733 \cdot 10^{-4}$ & $9.048 \cdot 10^{-4}$ & $2.456 \cdot 10^{-4}$ & $7.420 \cdot 10^{-5}$ \\
$10^{-3}$ & $3.315 \cdot 10^{-4}$ & $1.569 \cdot 10^{-2}$ & $4.616 \cdot 10^{-3}$ & $3.591 \cdot 10^{-3}$ & $1.072 \cdot 10^{-3}$ \\
$10^{-4}$ & $7.513 \cdot 10^{-4}$ & $2.369 \cdot 10^{-2}$ & $1.571 \cdot 10^{-3}$ & $9.581 \cdot 10^{-5}$ & $1.722 \cdot 10^{-4}$ \\
$10^{-5}$ & $8.031 \cdot 10^{-4}$ & $1.478 \cdot 10^{-2}$ & $1.076 \cdot 10^{-3}$ & $2.061 \cdot 10^{-4}$ & $6.661 \cdot 10^{-4}$ \\
\hline
\end{tabular}

Table 5.1.: Minimum inf-sup constants $\beta_{N}(\mu)$ (see (2.17)) evaluated using (a) Algorithm 1, (b) Algorithm 2, (c) Algorithm 3, and (d) Algorithm 4 (see $\$ 2.7$ and §4.7) for different values of $N$ and $\varepsilon$; the minimum is taken over 25 parameter values. 

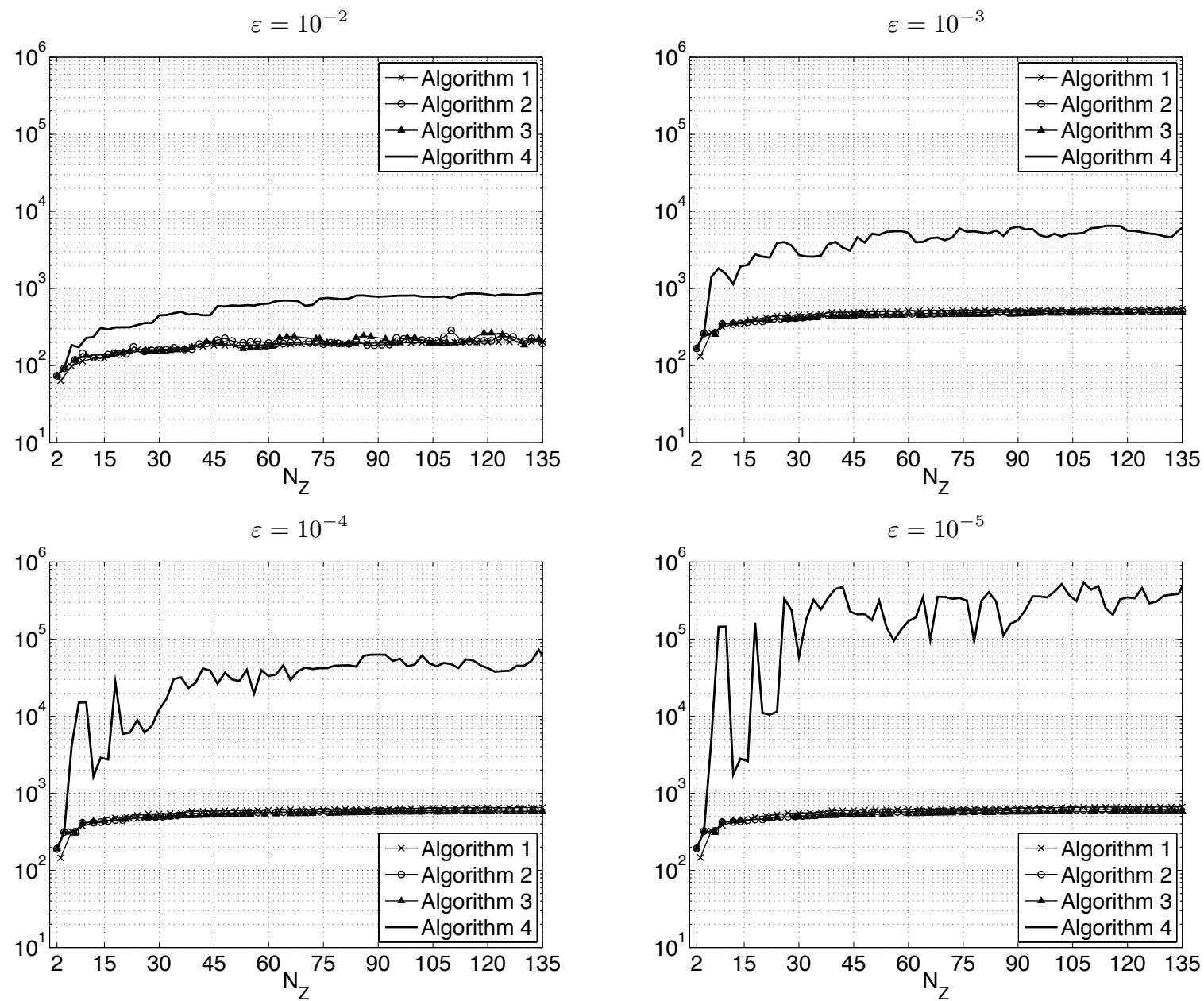

Figure 5.3.: Maximum condition number $\kappa_{N}^{\varepsilon}(\mu)$ (see (5.6)) of the reduced basis system matrix as a function of $N_{Z}$ for different values of $\varepsilon$ evaluated using Algorithm 1, Algorithm 2, Algorithm 3, and Algorithm 4 (see $\S 2.7$ and $\S 4.7$ ); the maximum is taken over 25 parameter values. 
In addition to the inf-sup constants $\beta_{N}(\mu)$, we also consider the condition number

$$
\kappa_{N}^{\varepsilon}(\mu) \equiv \frac{\sigma_{N}^{\varepsilon, \max }(\mu)}{\sigma_{N}^{\varepsilon, \min }(\mu)}, \quad \varepsilon>0, \quad \forall \mu \in \mathcal{D}, N \in \mathbb{N}_{\max }
$$

associated with the reduced basis system $(4.15),(4.16)$; here, $\sigma_{N}^{\varepsilon, \max }(\mu)$ and $\sigma_{N}^{\varepsilon, \min }(\mu)$ denote the maximum and minimum singular values of the corresponding system matrix (see also Appendix A). Figure 5.3 presents maximum values of $\kappa_{N}^{\varepsilon}(\mu)$ as a function of $N_{Z}$ for different values of $\varepsilon$. We observe that the penalty term has a significant impact on the condition number of the reduced basis system matrix: As $\varepsilon$ decreases and we approach the nonpenalized problem, values of $\kappa_{N}^{\varepsilon}(\mu)$ increase. In case of Algorithm 1, Algorithm 2, and Algorithm 3, this correlation is fairly weak and condition numbers seem to level off at a maximum of roughly 700. In case of Algorithm 4, the situation is different: Here, values of $\kappa_{N}^{\varepsilon}(\mu)$ explode and the reduced basis system becomes ill-conditioned as the penalty parameter $\varepsilon$ tends towards zero.

Accordingly reflected in our reduced basis approximations, this behavior manifests in practice what has been predicted by a priori stability estimates in $\S 4.3 .3$. We present in Fig. 5.4 the maximum relative errors $\left\|e_{N}^{u, \varepsilon}(\mu)\right\|_{X} /\left\|u^{\varepsilon}(\mu)\right\|_{X}$ and $\left\|e_{N}^{p, \varepsilon}(\mu)\right\|_{Y} /\left\|p^{\varepsilon}(\mu)\right\|_{Y}$ (see (4.18)) in the reduced basis velocity and pressure approximations using Algorithm 4 for different values of the penalty parameter $\varepsilon$; a direct comparison with the other sampling procedures is then given in Fig. 5.5 and Fig. 5.6. In case of Algorithm 1, Algorithm 2, and Algorithm 3, the velocity approximation spaces are rich enough to ensure stable reduced basis approximations whose convergence is not affected by the penalty parameter $\varepsilon$; for different values of $\varepsilon$, we obtain approximations $u_{N}^{\varepsilon}(\mu)$ for $u^{\varepsilon}(\mu)$ (see Fig. 5.5) and $p_{N}^{\varepsilon}(\mu)$ for $p^{\varepsilon}(\mu)$ (see Fig. 5.6) that are equally accurate and rapidly convergent. In case of Algorithm 4

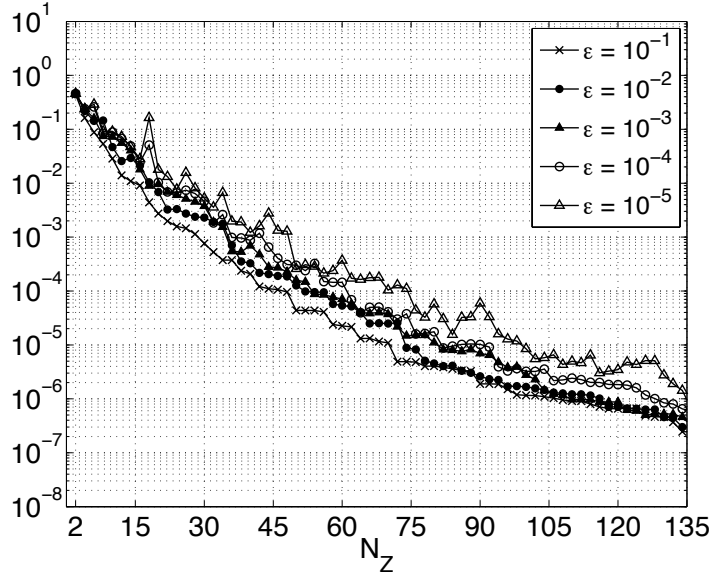

(a)

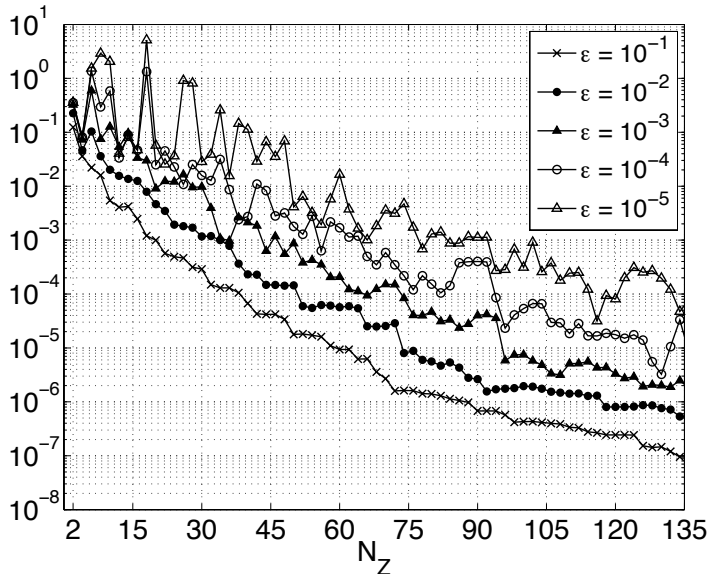

(b)

Figure 5.4.: Numerical results for Algorithm 4 (see $\S 4.7)$ for different values of $\varepsilon$ : Maximum relative errors (a) $\left\|e_{N}^{u, \varepsilon}(\mu)\right\|_{X} /\left\|u^{\varepsilon}(\mu)\right\|_{X}$ and (b) $\left\|e_{N}^{p, \varepsilon}(\mu)\right\|_{Y} /\left\|p^{\varepsilon}(\mu)\right\|_{Y}$ (see (4.18)) are shown as functions of $N_{Z}$; the maximum is taken over 25 parameter values. 

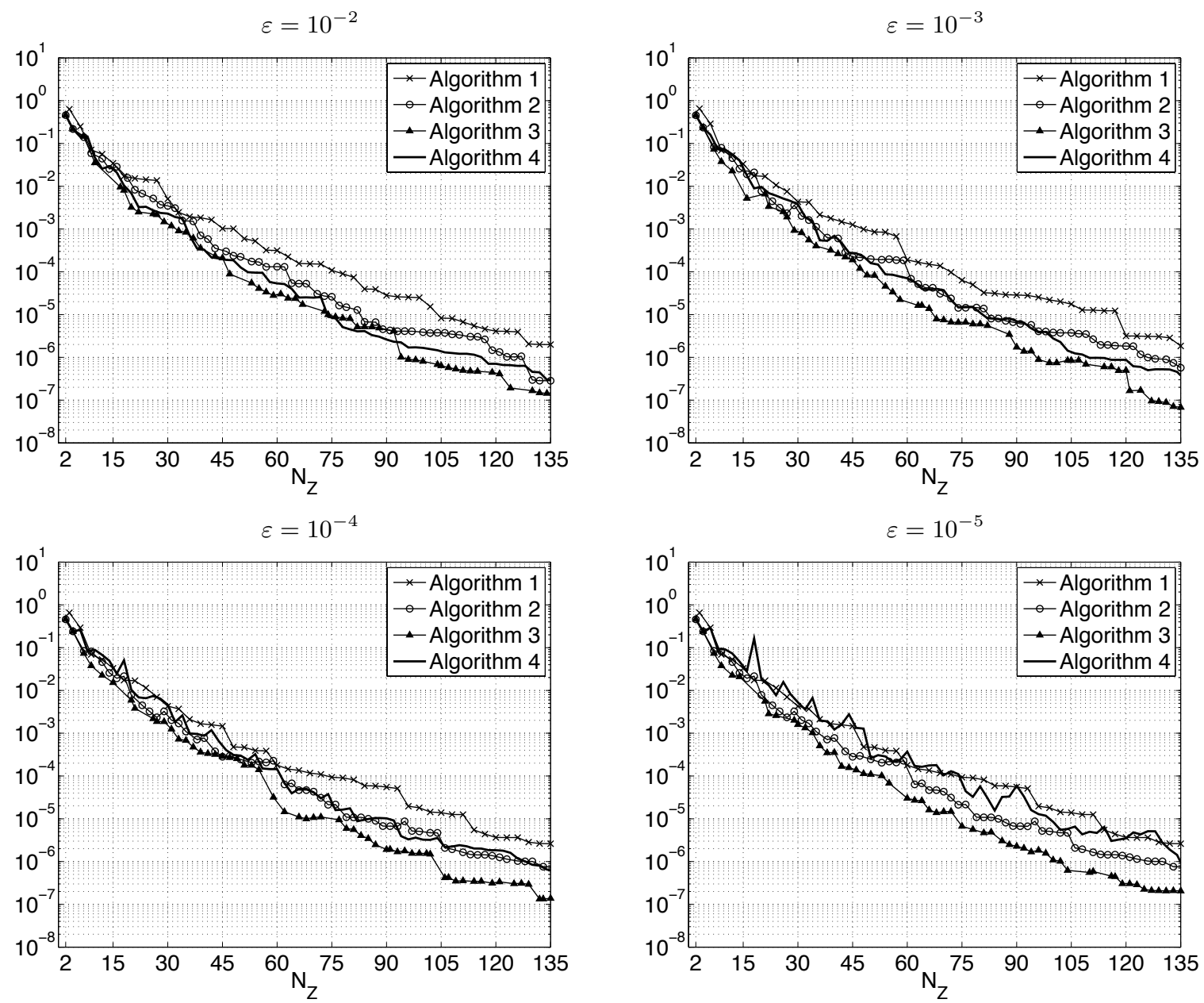

Figure 5.5.: Maximum relative error $\left\|e_{N}^{u, \varepsilon}(\mu)\right\|_{X} /\left\|u^{\varepsilon}(\mu)\right\|_{X}$ (see (4.18)) as a function of $N_{Z}$ for different values of $\varepsilon$ evaluated using Algorithm 1, Algorithm 2, Algorithm 3, and Algorithm 4 (see $\S 2.7$ and $\S 4.7$ ); the maximum is taken over 25 parameter values. 

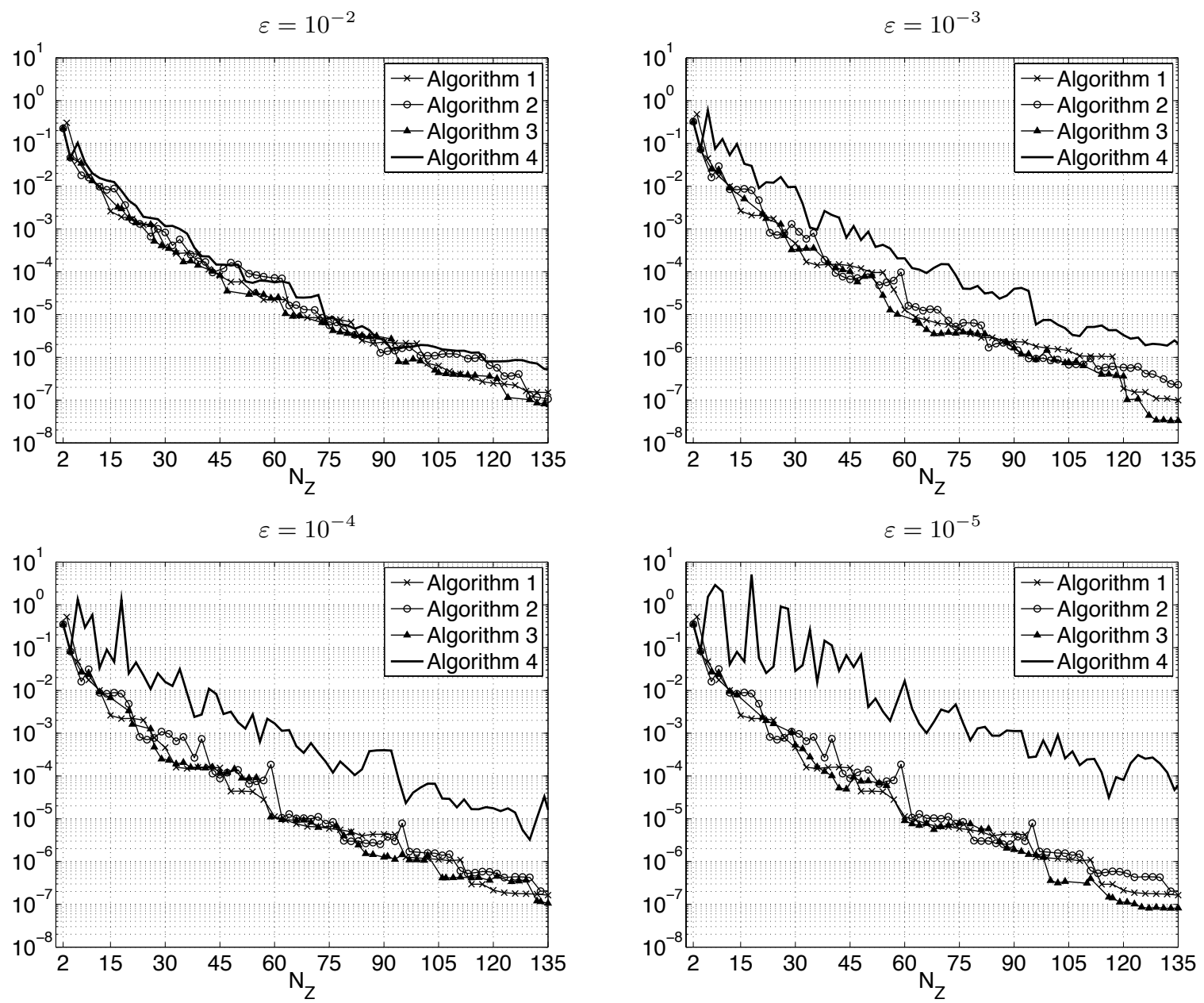

Figure 5.6.: Maximum relative error $\left\|e_{N}^{p, \varepsilon}(\mu)\right\|_{Y} /\left\|p^{\varepsilon}(\mu)\right\|_{Y}$ (see (4.18)) as a function of $N_{Z}$ for different values of $\varepsilon$ evaluated using Algorithm 1, Algorithm 2, Algorithm 3, and Algorithm 4 (see $\S 2.7$ and $\S 4.7$ ); the maximum is taken over 25 parameter values. 
where the inf-sup constants $\beta_{N}(\mu)$ associated with $X_{N}$ and $Y_{N}$ are comparatively small, convergence of the reduced basis approximations is remarkably good in the case of $\varepsilon \geq 10^{-2}$ but deteriorates for penalty parameters approaching zero (see Fig. 5.4). However, we note that this behavior is in practice much more prominent for the pressure than for the velocity approximations: For $\varepsilon \leq 10^{-3}$, errors in the approximations $p_{N}^{\varepsilon}(\mu)$ are much larger than in case of the other sampling procedures (see Fig. 5.6) whereas the approximations $u_{N}^{\varepsilon}(\mu)$ are still very accurate (see Fig. 5.4(a) and Fig. 5.5).

Again, we observe that stabilizing adaptively pays off: Compared to the standard greedy procedure in Algorithm 1, Algorithm 2 and Algorithm 3 achieve reduced basis approximations that are for the pressure equally well (see Fig. 5.6) but for the velocity noticeably better (see Fig. 5.5).

\subsubsection{A Posteriori Error Bounds}

We now investigate how the error bounds $\Delta_{N}^{\varepsilon}(\mu)$ developed in $\S 4.4$ perform in practice.

The SCM (see $\$ 4.6 .2$ and §2.6.2) enables the (Online-)efficient estimation of the coercivity constants $\alpha_{a}(\mu)$ and $\alpha_{c}(\mu)$ (see (2.10) and (4.12)). We here apply the method for $M_{\alpha}=\infty$ and an exhaustive sample $\Xi \subset \mathcal{D}$ of size $|\Xi|=4,225$. Setting further $\delta_{\text {tol }}^{\mathrm{SCM}}=0.01$, we obtain $K_{\max }=35$ for the coercivity constants $\alpha_{a}(\mu)$, and $K_{\max }=4$ for the coercivity constants $\alpha_{c}(\mu)$. We receive very accurate (Online-)efficient lower bounds $\alpha_{a}^{\mathrm{LB}}(\mu)$ and $\alpha_{c}^{\mathrm{LB}}(\mu)$ (see Fig. 3.14 in $\S 3.3 .4$ and Fig. 5.7), providing a posteriori error bounds $\Delta_{N}^{\varepsilon}(\mu)$ that essentially coincide with their values based on the evaluation of the exact constants $\alpha_{a}(\mu)$ and $\alpha_{c}(\mu)$.

In Fig. 5.8, Fig. 5.9, and Fig. 5.10, we now show the error $\left\|e_{N}^{\varepsilon}(\mu)\right\|_{Z, \varepsilon, \mu}$ (see (4.18), (4.19)) and the associated reduced basis a posteriori error bound $\Delta_{N}^{\varepsilon}(\mu)$ (see (4.33)) normalized relative to $\left\|\left(u^{\varepsilon}(\mu), p^{\varepsilon}(\mu)\right)\right\|_{Z, \varepsilon, \mu}$ evaluated using Algorithm 1, Algorithm 2, and Algorithm 3, respectively, for different values of the penalty parameter $\varepsilon$. We see that for $\varepsilon=10^{-2}$, the error bounds are tight and the effectivities are relatively small; however, the associated truth
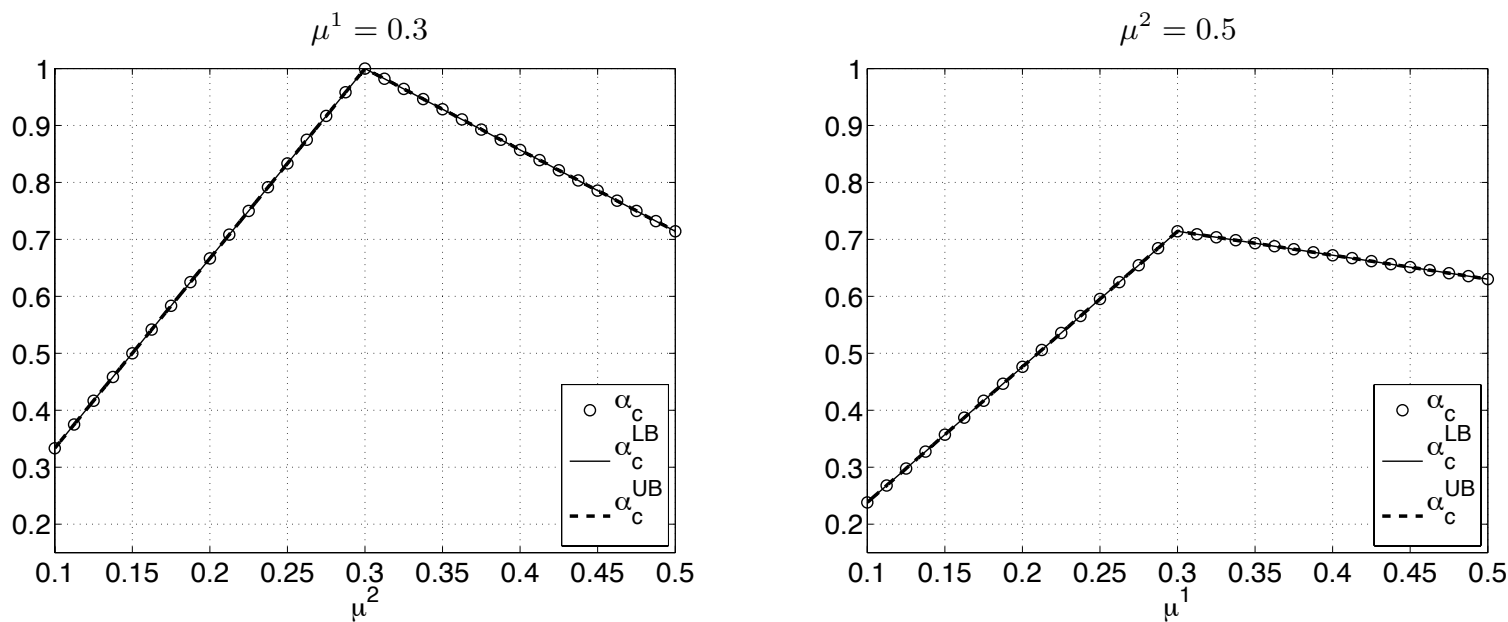

Figure 5.7.: SCM lower and upper bounds $\alpha_{c}^{\mathrm{LB}}(\mu)$ and $\alpha_{c}^{\mathrm{UB}}(\mu)$ for the coercivity constants $\alpha_{c}(\mu)$ (see $\left.(4.12),(4.29)\right)$ where $|\Xi|=4,225, M_{\alpha}=\infty, \delta_{\text {tol }}^{\mathrm{SCM}}=0.01$, and $K_{\max }=4$. 

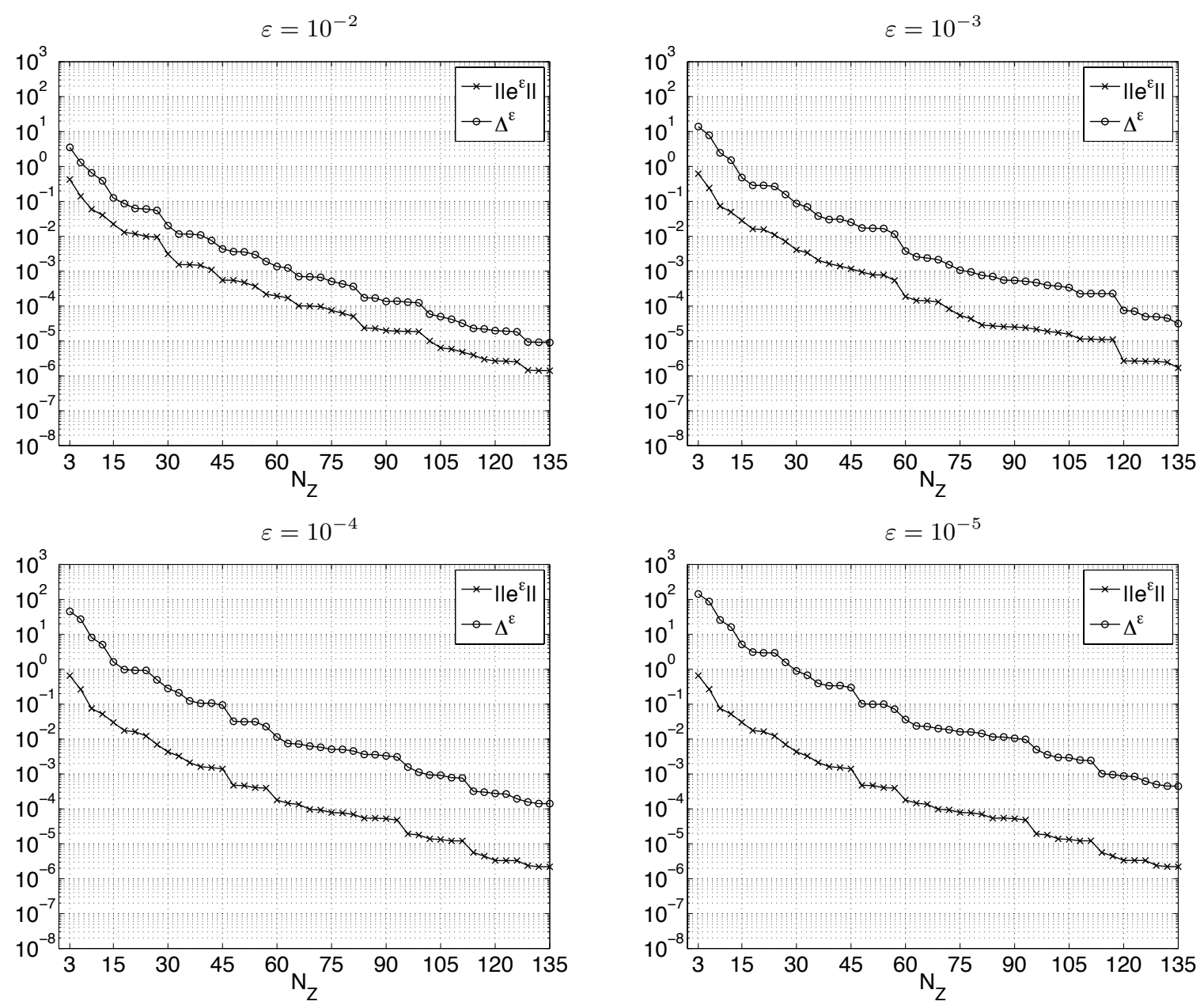

Figure 5.8.: Maximum relative error $\left\|e_{N}^{\varepsilon}(\mu)\right\|_{Z, \varepsilon, \mu} /\left\|\left(u^{\varepsilon}(\mu), p^{\varepsilon}(\mu)\right)\right\|_{Z, \varepsilon, \mu}$ (see (4.18), (4.19)) and maximum relative error bound $\Delta_{N}^{\varepsilon}(\mu) /\left\|\left(u^{\varepsilon}(\mu), p^{\varepsilon}(\mu)\right)\right\|_{Z, \varepsilon, \mu}$ (see (4.33)) as functions of $N_{Z}$ for different values of $\varepsilon$ evaluated using Algorithm 1 (see §2.7); the maximum is taken over 25 parameter values. 

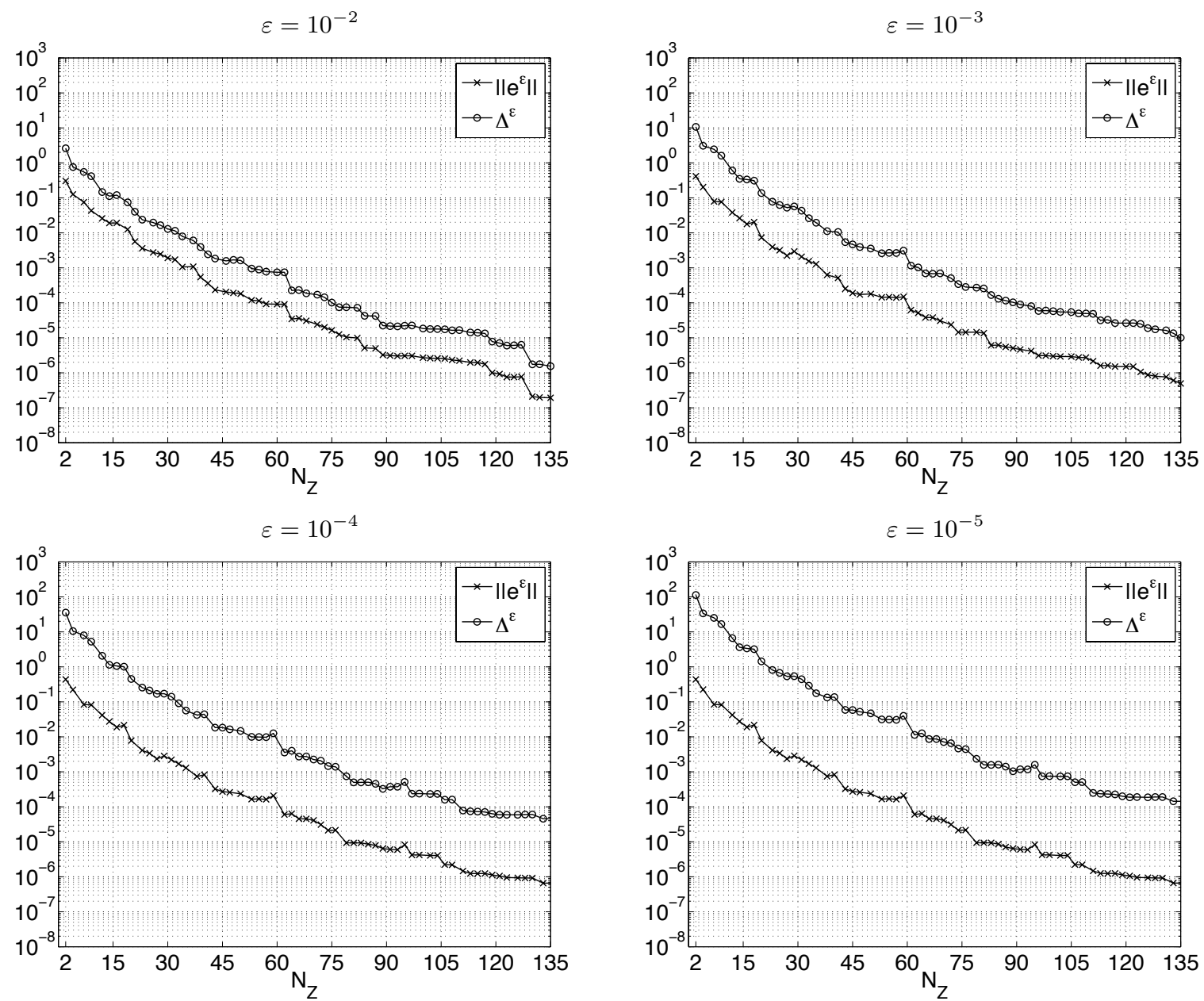

Figure 5.9.: Maximum relative error $\left\|e_{N}^{\varepsilon}(\mu)\right\|_{Z, \varepsilon, \mu} /\left\|\left(u^{\varepsilon}(\mu), p^{\varepsilon}(\mu)\right)\right\|_{Z, \varepsilon, \mu}$ (see (4.18), (4.19)) and maximum relative error bound $\Delta_{N}^{\varepsilon}(\mu) /\left\|\left(u^{\varepsilon}(\mu), p^{\varepsilon}(\mu)\right)\right\|_{Z, \varepsilon, \mu}$ (see (4.33)) as functions of $N_{Z}$ for different values of $\varepsilon$ evaluated using Algorithm 2 (see §2.7); the maximum is taken over 25 parameter values. 

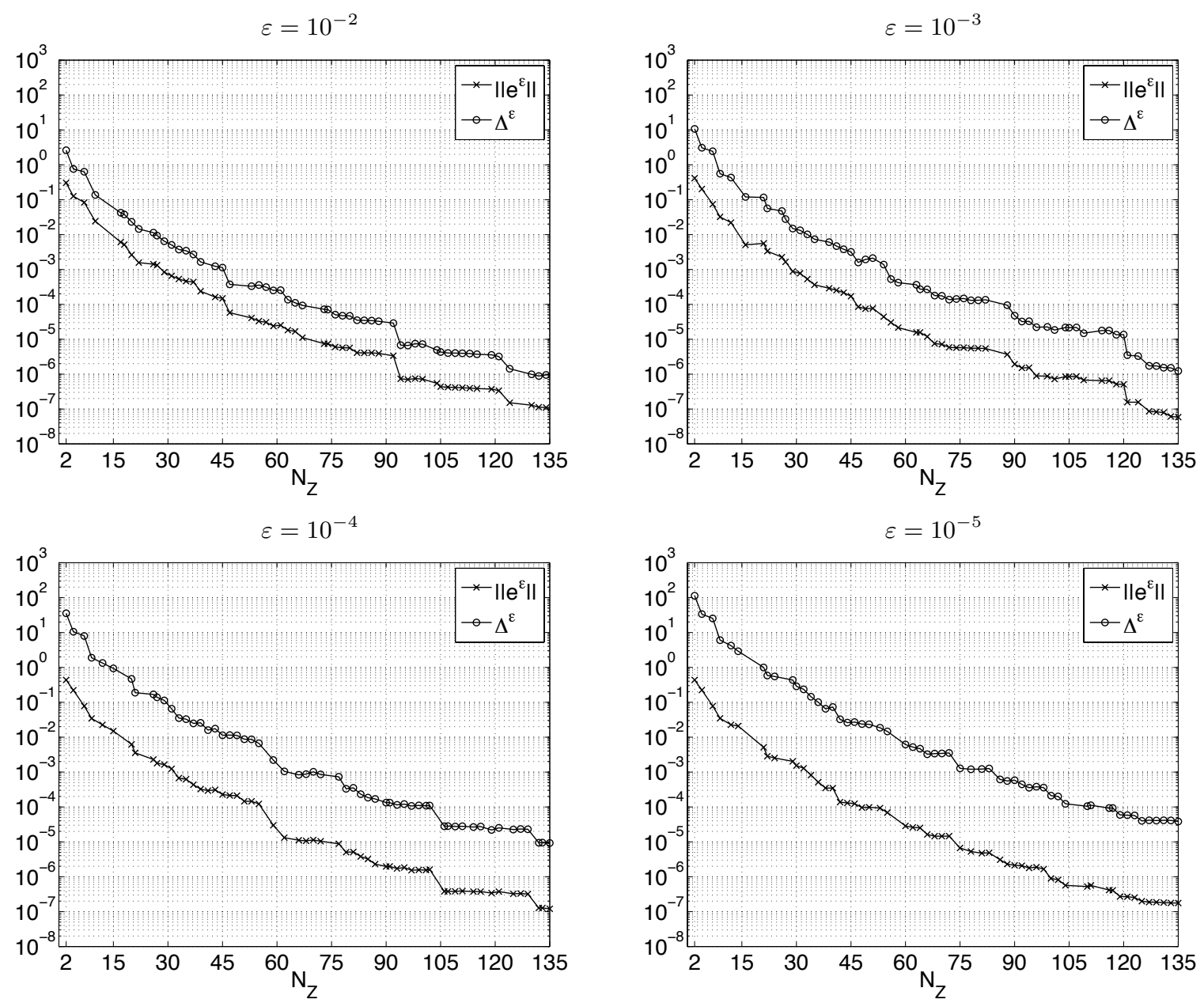

Figure 5.10.: Maximum relative error $\left\|e_{N}^{\varepsilon}(\mu)\right\|_{Z, \varepsilon, \mu} /\left\|\left(u^{\varepsilon}(\mu), p^{\varepsilon}(\mu)\right)\right\|_{Z, \varepsilon, \mu}$ (see (4.18), (4.19)) and maximum relative error bound $\Delta_{N}^{\varepsilon}(\mu) /\left\|\left(u^{\varepsilon}(\mu), p^{\varepsilon}(\mu)\right)\right\|_{Z, \varepsilon, \mu}$ (see (4.33)) as functions of $N_{Z}$ for different values of $\varepsilon$ evaluated using Algorithm 3 (see §2.7); the maximum is taken over 25 parameter values. 
(a) Algorithm 1

\begin{tabular}{c|c|c|c|c}
\hline$N$ & $\varepsilon=10^{-2}$ & $\varepsilon=10^{-3}$ & $\varepsilon=10^{-4}$ & $\varepsilon=10^{-5}$ \\
\hline 5 & $1.163 \cdot 10^{1}$ & $3.357 \cdot 10^{1}$ & $1.035 \cdot 10^{2}$ & $3.273 \cdot 10^{2}$ \\
10 & $1.149 \cdot 10^{1}$ & $3.407 \cdot 10^{1}$ & $1.065 \cdot 10^{2}$ & $3.368 \cdot 10^{2}$ \\
15 & $1.150 \cdot 10^{1}$ & $3.353 \cdot 10^{1}$ & $1.027 \cdot 10^{2}$ & $3.248 \cdot 10^{2}$ \\
20 & $1.030 \cdot 10^{1}$ & $3.515 \cdot 10^{1}$ & $1.111 \cdot 10^{2}$ & $3.511 \cdot 10^{2}$ \\
25 & $1.300 \cdot 10^{1}$ & $3.224 \cdot 10^{1}$ & $1.108 \cdot 10^{2}$ & $3.504 \cdot 10^{2}$ \\
30 & $1.171 \cdot 10^{1}$ & $3.145 \cdot 10^{1}$ & $9.657 \cdot 10^{1}$ & $3.053 \cdot 10^{2}$ \\
35 & $1.041 \cdot 10^{1}$ & $3.435 \cdot 10^{1}$ & $1.129 \cdot 10^{2}$ & $3.572 \cdot 10^{2}$ \\
40 & $1.068 \cdot 10^{1}$ & $3.456 \cdot 10^{1}$ & $1.017 \cdot 10^{2}$ & $3.217 \cdot 10^{2}$ \\
\hline
\end{tabular}

(b) Algorithm 2

\begin{tabular}{c|c|c|c|c}
\hline$N$ & $\varepsilon=10^{-2}$ & $\varepsilon=10^{-3}$ & $\varepsilon=10^{-4}$ & $\varepsilon=10^{-5}$ \\
\hline 5 & $1.167 \cdot 10^{1}$ & $3.277 \cdot 10^{1}$ & $1.040 \cdot 10^{2}$ & $3.288 \cdot 10^{2}$ \\
10 & $9.699 \cdot 10^{0}$ & $3.176 \cdot 10^{1}$ & $9.979 \cdot 10^{1}$ & $3.153 \cdot 10^{2}$ \\
15 & $9.903 \cdot 10^{0}$ & $3.259 \cdot 10^{1}$ & $1.046 \cdot 10^{2}$ & $3.308 \cdot 10^{2}$ \\
20 & $9.982 \cdot 10^{0}$ & $3.076 \cdot 10^{1}$ & $9.646 \cdot 10^{1}$ & $3.049 \cdot 10^{2}$ \\
25 & $9.710 \cdot 10^{0}$ & $3.110 \cdot 10^{1}$ & $9.811 \cdot 10^{1}$ & $3.107 \cdot 10^{2}$ \\
30 & $9.771 \cdot 10^{0}$ & $3.727 \cdot 10^{1}$ & $1.260 \cdot 10^{2}$ & $3.977 \cdot 10^{2}$ \\
35 & $1.070 \cdot 10^{1}$ & $2.899 \cdot 10^{1}$ & $8.831 \cdot 10^{1}$ & $2.790 \cdot 10^{2}$ \\
40 & $1.072 \cdot 10^{1}$ & $2.911 \cdot 10^{1}$ & $9.391 \cdot 10^{1}$ & $2.964 \cdot 10^{2}$ \\
\hline
\end{tabular}

(c) Algorithm 3

\begin{tabular}{c|c|c|c|c}
\hline$N$ & $\varepsilon=10^{-2}$ & $\varepsilon=10^{-3}$ & $\varepsilon=10^{-4}$ & $\varepsilon=10^{-5}$ \\
\hline 5 & $1.209 \cdot 10^{1}$ & $3.486 \cdot 10^{1}$ & $1.051 \cdot 10^{2}$ & $3.313 \cdot 10^{2}$ \\
10 & $1.249 \cdot 10^{1}$ & $4.018 \cdot 10^{1}$ & $1.215 \cdot 10^{2}$ & $3.155 \cdot 10^{2}$ \\
15 & $1.191 \cdot 10^{1}$ & $3.548 \cdot 10^{1}$ & $9.780 \cdot 10^{1}$ & $3.327 \cdot 10^{2}$ \\
20 & $1.207 \cdot 10^{1}$ & $3.281 \cdot 10^{1}$ & $1.121 \cdot 10^{2}$ & $3.229 \cdot 10^{2}$ \\
25 & $1.083 \cdot 10^{1}$ & $3.473 \cdot 10^{1}$ & $1.094 \cdot 10^{2}$ & $3.539 \cdot 10^{2}$ \\
30 & $1.160 \cdot 10^{1}$ & $3.322 \cdot 10^{1}$ & $1.102 \cdot 10^{2}$ & $3.623 \cdot 10^{2}$ \\
35 & $1.146 \cdot 10^{1}$ & $3.073 \cdot 10^{1}$ & $1.061 \cdot 10^{2}$ & $4.799 \cdot 10^{2}$ \\
40 & $1.056 \cdot 10^{1}$ & $3.629 \cdot 10^{1}$ & $9.906 \cdot 10^{1}$ & $3.742 \cdot 10^{2}$ \\
\hline
\end{tabular}

Table 5.2.: Maximum effectivities $\eta_{N}^{\varepsilon}(\mu)$ (see (4.37)) for several values of $N$ and $\varepsilon$ evaluated using (a) Algorithm 1, (b) Algorithm 2, and (c) Algorithm 3 (see §2.7); the maximum is taken over 25 parameter values.

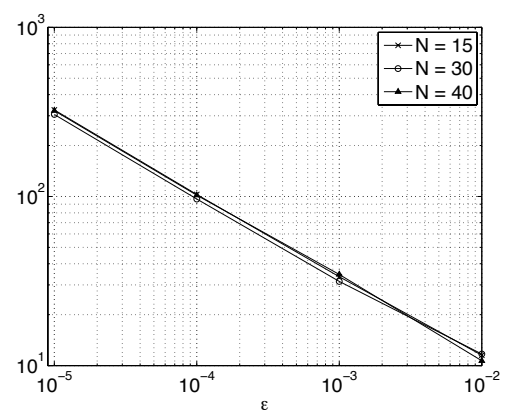

(a)

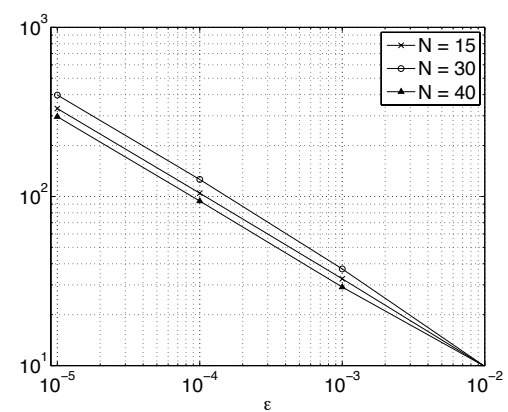

(b)

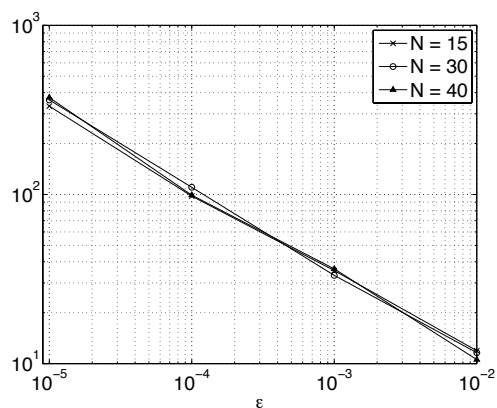

(c)

Figure 5.11.: Maximum effectivities $\eta_{N}^{\varepsilon}(\mu)$ (see (4.37)) as a function of $\varepsilon$ for different values of $N$ evaluated using (a) Algorithm 1, (b) Algorithm 2, and (c) Algorithm 3 (see §2.7); the maximum is taken over 25 parameter values. 
finite element approximation may not be sufficiently accurate (see Fig. 5.2). As we decrease $\varepsilon$, we obtain a more accurate truth approximation, but the reduced basis error bounds become less sharp. Nevertheless, these results show that even for relatively small values of the penalty parameter, the effectivities remain reasonably small, and the dependence on $\varepsilon$ is relatively weak as predicted by Corollary 4.4.2 and Corollary 4.4.3. To further quantify this statement, we present in Table 5.2(a), (b), and (c) the effectivities associated with $\Delta_{N}^{\varepsilon}(\mu)$ for several values of $N$ and $\varepsilon$ evaluated using Algorithm 1, Algorithm 2, and Algorithm 3, respectively. We note that for all three methods, the effectivities remain more or less constant with $N$. Furthermore, we show in Fig. 5.11 the dependence of the effectivities on the penalty parameter; we clearly observe the $O\left(\frac{1}{\sqrt{\varepsilon}}\right)$-dependence predicted by Corollary 4.4.2 and Corollary 4.4.3. The effects of the penalty parameter on the effectivities are thus relatively benign, and we obtain meaningful bounds for reasonably small values of $\varepsilon$. In particular, even at larger effectivities, the error bounds reflect the behavior of the actual error (see Fig. 5.8, Fig. 5.9, and Fig. 5.10), and our greedy sampling processes perform successfully as they are able to choose the parameter snapshots properly (i.e., where the error is largest).

\subsubsection{Computation Times}

We now discuss the Online computation times. The following timing tests were performed on a $2.66 \mathrm{GHz}$ Intel Core 2 Duo processor. For comparison, once the $\mu$-independent parts in (2.7) have been formed, direct computation of the truth approximation $\left(u^{\varepsilon}(\mu), p^{\varepsilon}(\mu)\right)$ (i.e., assembly and solution of (2.13)) takes on average 5.7 seconds. Again, the rigorous and efficient error bounds $\Delta_{N}^{\varepsilon}(\mu)$ allow us to choose the reduced basis system dimension $N_{Z}$ just large enough to obtain a desired accuracy.

For the penalty parameter $\varepsilon=10^{-2}$, the error bounds are sharp with effectivities of approximately 11 (see Table 5.2). If we construct the reduced basis approximations spaces by using the standard greedy procedure given in Algorithm 1, we then need $N_{Z}=42$ basis functions to achieve a prescribed accuracy of roughly $1 \%$ or better in the reduced basis approximations $\left(u_{N}^{\varepsilon}(\mu), p_{N}^{\varepsilon}(\mu)\right)$ (see Fig. 5.8). Once the database has been loaded, the Online calculation of $\left(u_{N}^{\varepsilon}(\mu), p_{N}^{\varepsilon}(\mu)\right)$ (i.e., assembly and solution of (4.14)) and $\Delta_{N}^{\varepsilon}(\mu)$ for any new value of $\mu \in \mathcal{D}$ takes on average 0.21 and 8.94 milliseconds, respectively, which is in total roughly 600 times faster than direct computation of the truth approximation. In case of Algorithm 3, the same accuracy is achieved for $N_{Z}=27$ (see Fig. 5.10); the Online calculation of $\left(u_{N}^{\varepsilon}(\mu), p_{N}^{\varepsilon}(\mu)\right)$ and $\Delta_{N}^{\varepsilon}(\mu)$ then takes on average 0.1 and 3.87 milliseconds, respectively, and is thus roughly 1,400 times faster than direct computation of the truth approximation.

We now set $\varepsilon=10^{-5}$. As they become more pessimistic with decreasing values of $\varepsilon$, the error bounds here dictate larger system dimensions at which they guarantee the same order of accuracy. Using Algorithm 1, we need $N_{Z}=93$ to achieve an accuracy of roughly $1 \%$ or better in the reduced basis approximations $\left(u_{N}^{\varepsilon}(\mu), p_{N}^{\varepsilon}(\mu)\right)$; the Online calculation of $\left(u_{N}^{\varepsilon}(\mu), p_{N}^{\varepsilon}(\mu)\right)$ and $\Delta_{N}^{\varepsilon}(\mu)$ for any new value of $\mu \in \mathcal{D}$ then takes on average 1.12 and 42.1 milliseconds, respectively, which is in total roughly 130 times faster than direct computation of the truth approximation. Using Algorithm 3, we need $N_{Z}=60$; the Online calculation of $\left(u_{N}^{\varepsilon}(\mu), p_{N}^{\varepsilon}(\mu)\right)$ and $\Delta_{N}^{\varepsilon}(\mu)$ then takes on average 0.4 and 16.76 milliseconds, 
respectively, which is in total roughly 330 times faster than direct computation of the truth approximation. Therefore, even for small penalty parameters $\varepsilon$, accurate approximations are guaranteed at significant Online savings.

Detailed computation times for different values of $\varepsilon$ are given in Table 5.3.

(a) $\varepsilon=10^{-2}$

\begin{tabular}{c|c|c|c|c|c}
\hline Method & $N_{Z}$ & $N$ & $\left(u_{N}^{\varepsilon}(\mu), p_{N}^{\varepsilon}(\mu)\right)$ & $\Delta_{N}^{\varepsilon}(\mu)$ & Total \\
\hline Algorithm 1 & $42(66)$ & $14(22)$ & $0.21(0.54)$ & $8.94(21.53)$ & $9.14(22.07)$ \\
\hline Algorithm 2 & $34(53)$ & $15(23)$ & $0.13(0.30)$ & $5.43(12.67)$ & $5.56(12.97)$ \\
\hline Algorithm 3 & $27(47)$ & $10(19)$ & $0.10(0.24)$ & $3.87(10.36)$ & $3.97(10.59)$ \\
\hline
\end{tabular}

(b) $\varepsilon=10^{-3}$

\begin{tabular}{c|c|c|c|c|c}
\hline Method & $N_{Z}$ & $N$ & $\left(u_{N}^{\varepsilon}(\mu), p_{N}^{\varepsilon}(\mu)\right)$ & $\Delta_{N}^{\varepsilon}(\mu)$ & Total \\
\hline Algorithm 1 & $60(78)$ & $20(26)$ & $0.42(0.78)$ & $17.84(29.90)$ & $18.26(30.69)$ \\
\hline Algorithm 2 & $43(65)$ & $19(29)$ & $0.19(0.47)$ & $8.39(18.77)$ & $8.58(19.25)$ \\
\hline Algorithm 3 & $35(56)$ & $14(23)$ & $0.14(0.35)$ & $5.96(14.39)$ & $6.09(14.74)$ \\
\hline
\end{tabular}

(c) $\varepsilon=10^{-4}$

\begin{tabular}{c|c|c|c|c|c}
\hline Method & $N_{Z}$ & $N$ & $\left(u_{N}^{\varepsilon}(\mu), p_{N}^{\varepsilon}(\mu)\right)$ & $\Delta_{N}^{\varepsilon}(\mu)$ & Total \\
\hline Algorithm 1 & $63(102)$ & $21(34)$ & $0.48(1.38)$ & $19.60(50.55)$ & $20.08(51.92)$ \\
\hline Algorithm 2 & $53(79)$ & $23(35)$ & $0.30(0.74)$ & $12.68(27.54)$ & $12.98(28.28)$ \\
\hline Algorithm 3 & $51(66)$ & $22(27)$ & $0.28(0.50)$ & $11.81(20.04)$ & $12.09(20.54)$ \\
\hline
\end{tabular}

(d) $\varepsilon=10^{-5}$

\begin{tabular}{c|c|c|c|c|c}
\hline Method & $N_{Z}$ & $N$ & $\left(u_{N}^{\varepsilon}(\mu), p_{N}^{\varepsilon}(\mu)\right)$ & $\Delta_{N}^{\varepsilon}(\mu)$ & Total \\
\hline Algorithm 1 & $93(117)$ & $31(39)$ & $1.12(1.79)$ & $42.10(66.10)$ & $43.22(67.89)$ \\
\hline Algorithm 2 & $66(97)$ & $29(44)$ & $0.48(1.12)$ & $19.44(40.69)$ & $19.92(41.80)$ \\
\hline Algorithm 3 & $60(86)$ & $24(35)$ & $0.40(0.91)$ & $16.76(33.63)$ & $17.16(34.53)$ \\
\hline
\end{tabular}

Table 5.3.: Average computation times in milliseconds for the Online evaluation of $\left(u_{N}^{\varepsilon}(\mu), p_{N}^{\varepsilon}(\mu)\right)$ (assembly and solution of (4.14)) and the error bounds $\Delta_{N}^{\varepsilon}(\mu)$ (see (4.33)): For (a) $\varepsilon=10^{-2}$, (b) $\varepsilon=10^{-3}$, (c) $\varepsilon=10^{-4}$, and (d) $\varepsilon=10^{-5}$, times are measured using either Algorithm 1, Algorithm 2, or Algorithm 3 (see $\S 2.7$ ) with a prescribed accuracy of at least $1 \%$ (resp., $0.1 \%$ ) for the reduced basis approximations $\left(u_{N}^{\varepsilon}(\mu), p_{N}^{\varepsilon}(\mu)\right)$.

\subsection{Concluding Remarks}

We present in this chapter numerical results for the reduced basis approach based on a penalty formulation proposed in Chapter 4. The method is applied to a perturbed Stokes flow in a parametrized microchannel.

As the computation of (Online-)efficient lower bounds to inf-sup stability constants is avoided, we obtain reduced basis approximations and rigorous a posteriori error bounds that are computed very easily. Numerical results show that the approximations for both the velocity and the pressure converge rapidly and that the error bounds are reasonably sharp. While the computation of inf-sup constants may be manageable for the Stokes problem, it becomes computationally even more intensive in case of the nonlinear Navier-Stokes 
equations. The penalty approach enables us to consider flow problems in parametrized domains with significantly less effort and thus provides a convenient way to also handle more complex geometric variations.

Clearly, the method also comes with several disadvantages. First, the introduced penalty term causes an additional error in the truth velocity and pressure approximations. For engineering problems of interest, these errors are generally acceptable but choosing an appropriate value of the penalty parameter $\varepsilon$ is a nontrivial issue. Second, the penalty term's regularizing effect is manifested in a significant impact on the condition number of the reduced basis system; yet, stable convergence cannot be guaranteed for reduced basis approximation spaces with small inf-sup constants (2.17). The problem can be resolved by an additional enrichment of the reduced basis approximation space for the primal variable: In this case, we obtain stable approximations whose convergence is not affected by the penalty parameter. Third, our a posteriori error bounds become more pessimistic as the penalty parameter $\varepsilon$ decreases and our truth approximations become more accurate. However, theoretical and numerical results show that these effects are relatively benign and we obtain meaningful and useful error bounds even for reasonably small values of $\varepsilon$.

Compared to former approaches, sampling procedures that recognize the need for stabilization adaptively again construct reduced basis approximation spaces that are computationally much more efficient. Even though the procedures used are not optimally suited for the penalty context (see Remark 4.7.1), they demonstrate the computational savings that are generally achievable; in the case of our Stokes model problem, we obtain computational savings of up to $40 \%$. Numerical results show that in the penalty context, the reduced basis inf-sup constants $\beta_{N}(\mu)$ are not necessarily appropriate indicators for an ill-conditioned system; well-suited adaptive sampling procedures should include the condition number $\kappa_{N}^{\varepsilon}(\mu)$. 



\section{Chapter 6}

\section{Approximation of the Instationary Stokes Equations}

\subsection{Introduction}

In this chapter, we provide an outlook on how the techniques presented in the previous part of this thesis may be extended to the time-dependent setting. To this end, we consider the instationary Stokes equations. We shall discuss both a method based on the standard formulation as well as a method based on a penalty approach, which combine approaches developed in $\S 2, \S 3$ and $\S 4, \S 5$ with current reduced basis techniques for parabolic problems (see, e.g., [37, 41, 47] and also [63, 64]). The analysis then shows how time integration affects the development of reduced basis a posteriori error bounds as well as the construction of computationally efficient reduced basis approximation spaces.

Starting from the standard mixed formulation of the instationary Stokes equations, we develop rigorous a posteriori error bounds for the reduced basis velocity approximations. As in the stationary case presented in $\S 2$ and $\S 3$, they involve the (Online-)estimation of coercivity, continuity, and inf-sup stability constants associated with the diffusion term and incompressibility constraint; in addition, they now also depend on continuity constants associated with the mass term. Employing a penalty formulation, we obtain rigorous upper bounds for the errors in both the velocity and pressure approximations. As in the stationary case presented in $\S 4$ and $\S 5$, they are computationally very efficient since they do not involve the estimation of inf-sup constants but only depend on coercivity constants associated with the diffusion and penalty terms; however, they again also depend on the penalty parameter such that associated effectivities increase as we approach the nonpenalized problem. To construct efficient reduced basis approximation spaces, we consider a POD greedy procedure (see $[37,46,47])$ that is coupled with adaptive stabilization techniques developed in $\S 2.7$. To demonstrate their performance in practice, the methods are then applied to a Stokes flow in a parametrized domain where evolution in time is induced by a time-dependent velocity profile on the inflow boundary.

The chapter is organized as follows: In $\S 6.2$, we introduce the general problem formulation and its "truth" approximation. We start from a time-discrete framework already that allows us to directly recover the settings discussed in $\S 2.2$ and $\S 4.2$; now, we have a saddle point problem associated with each time step. The time discretization scheme is here given by a 
backward Euler method. Section 6.3 defines our reduced basis approximation as the Galerkin projection onto a low-dimensional reduced basis approximation space. We develop rigorous a posteriori error bounds in $\S 6.4$. Again, reduced basis approximations and error bounds can be computed Online-efficiently as summarized in $§ 6.5$. This enables us to engage adaptive sampling processes for constructing computationally efficient reduced basis approximation spaces, which shall be discussed in $§ 6.6$. In $\S 6.7$, we introduce our instationary Stokes model problem. Numerical results in $\S 6.8$ then illustrate (i) the rapid convergence of reduced basis approximations, (ii) the performance of a posteriori error bounds with respect to sharpness, and (iii) computational efficiency. Finally, in $§ 6.9$, we give some concluding remarks.

\subsection{General Problem Statement}

\subsubsection{Formulation}

We assume the setting as introduced in $\S 2.2 .1$ and $\S 4.2 .1$. Furthermore, for any parameter $\mu \in \mathcal{D}$, let $m(\cdot, \cdot ; \mu): X_{\mathrm{e}} \times X_{\mathrm{e}} \rightarrow \mathbb{R}$ be a bilinear form that is symmetric, continuous, and positive definite,

$$
\begin{gathered}
\gamma_{m}^{\mathrm{e}}(\mu) \equiv \sup _{u \in X_{\mathrm{e}}} \sup _{v \in X_{\mathrm{e}}} \frac{m(u, v ; \mu)}{\|u\|_{X_{\mathrm{e}}}\|v\|_{X_{\mathrm{e}}}}<\infty, \quad \forall \mu \in \mathcal{D}, \\
m(v, v ; \mu)>0, \quad \forall 0 \neq v \in X_{\mathrm{e}}, \quad \forall \mu \in \mathcal{D},
\end{gathered}
$$

and which exhibits a $\mu$-affine representation analogous to $(2.7)$. For any $\mu \in \mathcal{D}, m(\cdot, \cdot ; \mu)$ then defines an inner product on $X_{\mathrm{e}}$; the associated norm shall be denoted by $\|\cdot\|_{\mu} \equiv \sqrt{m(\cdot, \cdot ; \mu)}$.

We further assume that we are given a time interval $[0, T], T>0$, and linear functionals $f(\cdot ; \mu) \in C^{0}\left(0, T ; X_{\mathrm{e}}^{\prime}\right)$ and $g(\cdot ; \mu) \in C^{0}\left(0, T ; Y_{\mathrm{e}}^{\prime}\right)$ for all $\mu \in \mathcal{D}$; for a vector space $V, C^{0}(0, T ; V)$ here denotes the space of $V$-valued functions of class $C^{0}$ with respect to $t \in[0, T]$. Throughout this thesis, we directly consider a time-discrete framework: We divide the time interval $[0, T]$ into $K$ subintervals of equal length $\Delta t \equiv T / K$, and define $t^{k} \equiv k \Delta t$ for all $k=0, \ldots, K$; for notational convenience, we also introduce $\mathbb{K} \equiv\{1, \ldots, K\}$ and $\mathbb{K}_{0} \equiv \mathbb{K} \cup\{0\}$. We then set $f^{k}(\cdot ; \mu) \equiv f\left(t^{k} ; \mu\right) \in X_{\mathrm{e}}^{\prime}$ and $g^{k}(\cdot ; \mu) \equiv g\left(t^{k} ; \mu\right) \in Y_{\mathrm{e}}^{\prime}$ for all $k \in \mathbb{K}_{0}, \mu \in \mathcal{D}$.

For $\varepsilon \geq 0$, we now consider the following "exact" - more precisely, semi-discrete problem resulting from a backward Euler method (see, e.g., [27, 42, 89, 102]): For any given $\mu \in \mathcal{D}$, we find $u_{\mathrm{e}}^{\varepsilon, k}(\mu) \in X_{\mathrm{e}}$ and $p_{\mathrm{e}}^{\varepsilon, k}(\mu) \in Y_{\mathrm{e}}, k \in \mathbb{K}$, such that $u_{\mathrm{e}}^{\varepsilon, 0}(\mu)=0^{1}$ and

$$
\begin{aligned}
\frac{1}{\Delta t} m\left(u_{\mathrm{e}}^{\varepsilon, k}(\mu)-u_{\mathrm{e}}^{\varepsilon, k-1}(\mu), v ; \mu\right) & \\
+a\left(u_{\mathrm{e}}^{\varepsilon, k}(\mu), v ; \mu\right)+b\left(v, p_{\mathrm{e}}^{\varepsilon, k}(\mu) ; \mu\right) & =f^{k}(v ; \mu), \quad \forall v \in X_{\mathrm{e}}, \quad k \in \mathbb{K} . \\
b\left(u_{\mathrm{e}}^{\varepsilon, k}(\mu), q ; \mu\right)-\varepsilon c\left(p_{\mathrm{e}}^{\varepsilon, k}(\mu), q ; \mu\right) & =g^{k}(q ; \mu), \quad \forall q \in Y_{\mathrm{e}},
\end{aligned}
$$

Even though we here use a common notation for simplicity in exposition, we point out that (6.3) states very different problems for $\varepsilon=0$ and $\varepsilon>0$, respectively. For $\varepsilon=0$, we also denote $u_{\mathrm{e}}^{k}(\mu) \equiv u_{\mathrm{e}}^{0, k}(\mu), k \in \mathbb{K}_{0}$, and $p_{\mathrm{e}}^{k}(\mu) \equiv p_{\mathrm{e}}^{0, k}(\mu), k \in \mathbb{K}$, for all $\mu \in \mathcal{D}$. For $\varepsilon>0$,

\footnotetext{
${ }^{1}$ We here assume zero initial conditions for simplicity; note that nonzero initial conditions can be handled as well without much difficulty (see [41]).
} 
corresponding to our discussions in Chapter 4, (6.3) can be considered as a perturbed or regularized version of the problem associated with $\varepsilon=0$; in this case, we therefore call $\left(u_{\mathrm{e}}^{\varepsilon, k}(\mu), p_{\mathrm{e}}^{\varepsilon, k}(\mu)\right), k \in \mathbb{K}$, also the penalty solution. Since these problems differ considerably in their general nature (see $\S 2$ and $\S 4$ ), we shall often treat them separately in the following analysis and explicitly distinguish between the two cases $\varepsilon=0$ and $\varepsilon>0$. From (6.2) and (2.3), the bilinear form $\frac{1}{\Delta t} m(\cdot, \cdot ; \mu)+a(\cdot, \cdot ; \mu)$ is coercive on $X_{\mathrm{e}}$ for any $\mu \in \mathcal{D}$. The problem (6.3) is thus uniquely solvable for $\left(u_{\mathrm{e}}^{k}(\mu), p_{\mathrm{e}}^{k}(\mu)\right), k \in \mathbb{K}$, and $\left(u_{\mathrm{e}}^{\varepsilon, k}(\mu), p_{\mathrm{e}}^{\varepsilon, k}(\mu)\right), k \in \mathbb{K}$, as a saddle point problem according to $\S 2.2 .1$ and $\S 4.2 .1$, respectively.

\subsubsection{Truth Approximation}

As in $\S 2.2 .2$ and $\S 4.2 .2$, we denote by $X$ and $Y$ finite-dimensional subspaces of $X_{\mathrm{e}}$ and $Y_{\mathrm{e}}$ satisfying the assumptions (2.8)-(2.11), (4.11), and (4.12). These in particular also inherit the inner product $m(\cdot, \cdot ; \mu)$ and associated norm $\|\cdot\|_{\mu}$,

$$
\begin{aligned}
\gamma_{m}(\mu) & \equiv \sup _{u \in X} \sup _{v \in X} \frac{m(u, v ; \mu)}{\|u\|_{X}\|v\|_{X}}=\sup _{v \in X} \frac{m(v, v ; \mu)}{\|v\|_{X}^{2}}<\infty, \\
m(v, v ; \mu) & >0, \quad \forall 0 \neq v \in X, \quad \forall \mu \in \mathcal{D} .
\end{aligned}
$$

Again, we denote by $\mathcal{N}$ the large dimension of the approximation space $Z=X \times Y$.

Our high-fidelity "truth" discretization for (6.3) now reads as follows: For $\varepsilon \geq 0$ and any given $\mu \in \mathcal{D}$, we find $u^{\varepsilon, k}(\mu) \in X$ and $p^{\varepsilon, k}(\mu) \in Y, k \in \mathbb{K}$, such that $u^{\varepsilon, 0}(\mu)=0$ and

$$
\begin{aligned}
\frac{1}{\Delta t} m\left(u^{\varepsilon, k}(\mu)-u^{\varepsilon, k-1}(\mu), v ; \mu\right) & \\
+a\left(u^{\varepsilon, k}(\mu), v ; \mu\right)+b\left(v, p^{\varepsilon, k}(\mu) ; \mu\right)=f^{k}(v ; \mu), & \forall v \in X, \\
b\left(u^{\varepsilon, k}(\mu), q ; \mu\right)-\varepsilon c\left(p^{\varepsilon, k}(\mu), q ; \mu\right)=g^{k}(q ; \mu), & \forall q \in Y,
\end{aligned}
$$

In case of $\varepsilon=0$, we also denote $u^{k}(\mu) \equiv u^{0, k}(\mu), k \in \mathbb{K}_{0}$, and $p^{k}(\mu) \equiv p^{0, k}(\mu), k \in \mathbb{K}$. As the exact problem in $\S 6.2 .1$, the problem (6.6) is uniquely solvable for $\left(u^{k}(\mu), p^{k}(\mu)\right), k \in \mathbb{K}$, and $\left(u^{\varepsilon, k}(\mu), p^{\varepsilon, k}(\mu)\right), k \in \mathbb{K}$, according to $\S 2.2 .2$ and $\S 4.2 .2$, respectively.

Let $A(\mu), B(\mu), B(\mu)^{t}$, and $C(\mu)$ be the linear operators associated with the bilinear forms $a(\cdot, \cdot ; \mu), b(\cdot, \cdot ; \mu)$, and $c(\cdot, \cdot ; \mu)$ as introduced in $\S 2.2 .2$ and $\S 4.2 .2$; accordingly, we also define the bounded linear operator $M(\mu): X \rightarrow X^{\prime}$ by

$$
\langle M(\mu) u, v\rangle=m(u, v ; \mu), \quad \forall u, v \in X .
$$

The system (6.6) may then be equivalently written as

$$
\begin{gathered}
M(\mu) \frac{u^{\varepsilon, k}(\mu)-u^{\varepsilon, k-1}(\mu)}{\Delta t}+A(\mu) u^{\varepsilon, k}(\mu)+B(\mu)^{t} p^{\varepsilon, k}(\mu)=f^{k}(\mu) \\
B(\mu) u^{\varepsilon, k}(\mu)-\varepsilon C(\mu) p^{\varepsilon, k}(\mu)=g^{k}(\mu) \quad \text { in } Y^{\prime}, \quad k \in \mathbb{K},
\end{gathered}
$$

where $\left.f^{k}(\mu) \equiv f^{k}(\cdot ; \mu)\right|_{X} \in X^{\prime}$ and $\left.g^{k}(\mu) \equiv g^{k}(\cdot ; \mu)\right|_{Y} \in Y^{\prime}$ for all $k \in \mathbb{K}_{0}$. 


\subsection{Reduced Basis Approximation}

We now turn to the reduced basis method, discussing the approximation procedure, rigorous a posteriori error estimators, and the construction of stable approximation spaces that capture the causality associated with the parameter dependence as well as with evolution in time.

Suppose that we are given a set of nested, low-dimensional reduced basis approximation subspaces $X_{N} \subset X_{N+1} \subset X$ and $Y_{N} \subset Y_{N+1} \subset Y, N \in \mathbb{N}_{\max } \equiv\left\{1, \ldots, N_{\max }\right\}$, then the reduced basis approximation is defined as the Galerkin projection with respect to the truth problem (6.6) onto these low-dimensional subspaces: For $\varepsilon \geq 0$ and any given $\mu \in \mathcal{D}$, we find $u_{N}^{\varepsilon, k}(\mu) \in X_{N}$ and $p_{N}^{\varepsilon, k}(\mu) \in Y_{N}, k \in \mathbb{K}$, such that $u_{N}^{\varepsilon, 0}(\mu)=0$ and

$$
\begin{aligned}
\frac{1}{\Delta t} m\left(u_{N}^{\varepsilon, k}(\mu)-u_{N}^{\varepsilon, k-1}(\mu), v_{N} ; \mu\right) & \\
+a\left(u_{N}^{\varepsilon, k}(\mu), v_{N} ; \mu\right)+b\left(v_{N}, p_{N}^{\varepsilon, k}(\mu) ; \mu\right)=f^{k}\left(v_{N} ; \mu\right), & \forall v_{N} \in X_{N}, \quad k \in \mathbb{K} . \\
b\left(u_{N}^{\varepsilon, k}(\mu), q_{N} ; \mu\right)-\varepsilon c\left(p_{N}^{\varepsilon, k}(\mu), q_{N} ; \mu\right)=g^{k}\left(q_{N} ; \mu\right), & \forall q_{N} \in Y_{N},
\end{aligned}
$$

Again, we denote $u_{N}^{k}(\mu) \equiv u_{N}^{0, k}(\mu), k \in \mathbb{K}_{0}$, and $p_{N}^{k}(\mu) \equiv p_{N}^{0, k}(\mu), k \in \mathbb{K}$. Written in operator notation, the discrete system (6.7) reads

$$
\begin{aligned}
\frac{1}{\Delta t} M_{N}(\mu) & \left(u_{N}^{\varepsilon, k}(\mu)-u_{N}^{\varepsilon, k-1}(\mu)\right) \\
+ & A_{N}(\mu) u_{N}^{\varepsilon, k}(\mu)+B_{N}(\mu)^{t} p_{N}^{\varepsilon, k}(\mu)=f_{N}^{k}(\mu) \text { in } X_{N}^{\prime}, \quad k \in \mathbb{K}, \\
B_{N}(\mu) u_{N}^{\varepsilon, k}(\mu)-\varepsilon C_{N}(\mu) p_{N}^{\varepsilon, k}(\mu)=g_{N}^{k}(\mu) \text { in } Y_{N}^{\prime}, &
\end{aligned}
$$

where $A_{N}(\mu), B_{N}(\mu), B_{N}(\mu)^{t}$, and $C_{N}(\mu)$ are the linear operators defined in $\S 2.3 .1$ and $\S 4.3 .1$, and $M_{N}(\mu): X_{N} \rightarrow X_{N}^{\prime}$ is given by

$$
\left\langle M_{N}(\mu) u_{N}, v_{N}\right\rangle=m\left(u_{N}, v_{N} ; \mu\right), \quad \forall u_{N}, v_{N} \in X_{N}, \quad \forall \mu \in \mathcal{D} ;
$$

moreover, $\left.f_{N}^{k}(\mu) \equiv f^{k}(\cdot ; \mu)\right|_{X_{N}} \in X_{N}^{\prime}$ and $\left.g_{N}^{k}(\mu) \equiv g^{k}(\cdot ; \mu)\right|_{Y_{N}} \in Y_{N}^{\prime}$ for all $k \in \mathbb{K}_{0}$.

The discrete reduced basis system now essentially behaves as in the stationary case: In case of $\varepsilon=0,(6.7)$ is uniquely solvable for $\left(u_{N}^{k}(\mu), p_{N}^{k}(\mu)\right), k \in \mathbb{K}$, if and only if the reduced basis approximation spaces $X_{N}, Y_{N}$ are stable (as defined in $\S 2.3 .2$ ); in case of $\varepsilon>0,(6.7)$ is uniquely solvable for $\left(u_{N}^{\varepsilon, k}(\mu), p_{N}^{\varepsilon, k}(\mu)\right), k \in \mathbb{K}$, for any choice of $X_{N}, Y_{N}$ (see $\S 4.3 .2$ ).

\subsection{A Posteriori Error Estimation}

We now develop upper bounds for the errors in our reduced basis approximations that are rigorous, sharp, and computationally efficient. As before, the errors shall be measured relative to the respective truth approximations.

In this section, we assume that the low-dimensional reduced basis spaces $X_{N}, Y_{N}$ are constructed such that for any given parameter $\mu \in \mathcal{D}$, a solution $\left(u_{N}^{\varepsilon, k}(\mu), p_{N}^{\varepsilon, k}(\mu)\right) \in X_{N} \times Y_{N}$, 
$k \in \mathbb{K}$, to (6.7) exists. For $\mu \in \mathcal{D}$, we then consider the errors

$$
\begin{aligned}
& e_{N}^{u}(\mu) \equiv\left(e_{N}^{u, k}(\mu)\right)_{k \in \mathbb{K}}, \text { where } e_{N}^{u, k}(\mu) \equiv u^{\varepsilon, k}(\mu)-u_{N}^{\varepsilon, k}(\mu) \in X, k \in \mathbb{K}, \\
& e_{N}^{p}(\mu) \equiv\left(e_{N}^{p, k}(\mu)\right)_{k \in \mathbb{K}}, \text { where } e_{N}^{p, k}(\mu) \equiv p^{\varepsilon, k}(\mu)-p_{N}^{\varepsilon, k}(\mu) \in Y, k \in \mathbb{K}, \\
& e_{N}^{\varepsilon}(\mu) \equiv\left(e_{N}^{\varepsilon, k}(\mu)\right)_{k \in \mathbb{K}}, \text { where } e_{N}^{\varepsilon, k}(\mu) \equiv\left(e_{N}^{u, k}(\mu), e_{N}^{p, k}(\mu)\right) \in Z, k \in \mathbb{K},
\end{aligned}
$$

in the reduced basis approximations $\left(u_{N}^{\varepsilon, k}(\mu), p_{N}^{\varepsilon, k}(\mu)\right), k \in \mathbb{K}$, with respect to the truth solution $\left(u^{\varepsilon, k}(\mu), p^{\varepsilon, k}(\mu)\right), k \in \mathbb{K}$; we note that in particular $e_{N}^{u, 0}(\mu) \equiv u^{\varepsilon, 0}(\mu)-u_{N}^{\varepsilon, 0}(\mu)=0$ from our initial conditions.

To formulate our reduced basis a posteriori error bounds, we again rely on the residuals associated with the reduced basis approximation $\left(u_{N}^{\varepsilon, k}(\mu), p_{N}^{\varepsilon, k}(\mu)\right), k \in \mathbb{K}$,

$$
\begin{aligned}
& r_{N}^{1, k}(\cdot ; \mu) \equiv f^{k}(\mu)-M(\mu) \frac{u_{N}^{\varepsilon, k}(\mu)-u_{N}^{\varepsilon, k-1}(\mu)}{\Delta t}-A(\mu) u_{N}^{\varepsilon, k}(\mu)-B(\mu)^{t} p_{N}^{\varepsilon, k}(\mu) \in X^{\prime}, \\
& r_{N}^{2, k}(\cdot ; \mu) \equiv g^{k}(\mu)-B(\mu) u_{N}^{\varepsilon, k}(\mu)+\varepsilon C(\mu) p_{N}^{\varepsilon, k}(\mu) \in Y^{\prime}
\end{aligned}
$$

for $k \in \mathbb{K}$ and $\mu \in \mathcal{D}$.

In the following analysis, we distinguish between the cases $\varepsilon=0$ and $\varepsilon>0$.

\subsection{1 $\varepsilon=0$}

We here derive rigorous upper bounds for the error $e_{N}^{u}(\mu)$ measured in the "spatio-temporal" energy norm

$$
\left\|\left(v^{j}\right)_{j \in \mathbb{K}}\right\|_{\ell^{2}(0, k ; X)} \equiv\left(\left\|v^{k}\right\|_{\mu}^{2}+\Delta t \sum_{j=1}^{k}\left\|v^{j}\right\|_{X, \mu}^{2}\right)^{1 / 2}, \quad\left(v^{j}\right)_{j \in \mathbb{K}} \subseteq X, \quad k \in \mathbb{K} .
$$

As before, our reduced basis a posteriori error bounds shall be formulated in terms of the dual norms of the residuals (6.10) and (6.11), and (Online-)efficient lower and upper bounds to the truth continuity, coercivity, and inf-sup stability constants (2.8), (2.10), and (2.11). In addition to (2.29) and (2.30), we also introduce computationally (Online-)efficient (lower and) upper bounds to the truth continuity constants (6.4),

$$
\gamma_{m}^{\mathrm{LB}}(\mu) \leq \gamma_{m}(\mu) \leq \gamma_{m}^{\mathrm{UB}}(\mu), \quad \forall \mu \in \mathcal{D} .
$$

We can now state the following result.

Proposition 6.4.1. For any given $\mu \in \mathcal{D}, N \in \mathbb{N}_{\max }, k \in \mathbb{K}$, and $\alpha_{a}^{\mathrm{LB}}(\mu), \gamma_{a}^{\mathrm{UB}}(\mu), \beta_{\mathrm{Br}}^{\mathrm{LB}}(\mu)$, $\gamma_{m}^{\mathrm{UB}}(\mu)$ satisfying (2.29), (2.30), and (6.13), we define

$$
\begin{aligned}
\Delta_{N}^{k}(\mu) \equiv\left[\Delta t \sum_{j=1}^{k} \frac{\left\|r_{N}^{1, j}(\cdot ; \mu)\right\|_{X^{\prime}}^{2}}{\alpha_{a}^{\mathrm{LB}}(\mu)}+\frac{2}{\beta_{\mathrm{Br}}^{\mathrm{LB}}(\mu)}\left(1+\frac{\gamma_{a}^{\mathrm{UB}}(\mu)}{\alpha_{a}^{\mathrm{LB}}(\mu)}\right)\left\|r_{N}^{1, j}(\cdot ; \mu)\right\|_{X^{\prime}}\left\|r_{N}^{2, j}(\cdot ; \mu)\right\|_{Y^{\prime}}\right. \\
\left.+\left(\frac{\gamma_{m}^{\mathrm{UB}}(\mu)}{\Delta t}+\frac{\left(\gamma_{a}^{\mathrm{UB}}(\mu)\right)^{2}}{\alpha_{a}^{\mathrm{LB}}(\mu)}\right) \frac{\left\|r_{N}^{2, j}(\cdot ; \mu)\right\|_{Y^{\prime}}^{2}}{\left(\beta_{\mathrm{Br}}^{\mathrm{LB}}(\mu)\right)^{2}}\right]^{1 / 2}
\end{aligned}
$$


Then, $\Delta_{N}^{k}(\mu)$ represents an upper bound for the error $e_{N}^{u}(\mu)$ measured in the "spatio-temporal" energy norm (6.12),

$$
\left\|e_{N}^{u}(\mu)\right\|_{\ell^{2}(0, k ; X)} \leq \Delta_{N}^{k}(\mu), \quad \forall k \in \mathbb{K}, \mu \in \mathcal{D}, N \in \mathbb{N}_{\max } .
$$

Proof. Let $\mu$ be any parameter in $\mathcal{D}, N \in \mathbb{N}_{\max }$, and $k \in \mathbb{K}$. For clarity of exposition, we suppress the argument $\mu$ in this proof.

Take any $1 \leq j \leq k$. From (6.10), (6.11), and (6.6), the errors $e_{N}^{u, j} \in X$ and $e_{N}^{p, j} \in Y$ satisfy the equations

$$
\begin{aligned}
\frac{1}{\Delta t} m\left(e_{N}^{u, j}-e_{N}^{u, j-1}, v\right)+a\left(e_{N}^{u, j}, v\right)+b\left(v, e_{N}^{p, j}\right) & =r_{N}^{1, j}(v), \quad \forall v \in X, \\
b\left(e_{N}^{u, j}, q\right) & =r_{N}^{2, j}(q), \quad \forall q \in Y .
\end{aligned}
$$

By the LBB inf-sup condition (2.11) and (6.16), we have

$$
\begin{aligned}
\beta_{\mathrm{Br}}\left\|e_{N}^{p, j}\right\|_{Y} & \leq \sup _{v \in X} \frac{b\left(v, e_{N}^{p, j}\right)}{\|v\|_{X}}=\sup _{v \in X} \frac{r_{N}^{1, j}(v)-a\left(e_{N}^{u, j}, v\right)-\frac{1}{\Delta t} m\left(e_{N}^{u, j}-e_{N}^{u, j-1}, v\right)}{\|v\|_{X}} \\
& \leq\left\|r_{N}^{1, j}\right\|_{X^{\prime}}+\gamma_{a}\left\|e_{N}^{u, j}\right\|_{X}+\frac{\sqrt{\gamma_{m}}}{\Delta t}\left\|e_{N}^{u, j}-e_{N}^{u, j-1}\right\|_{\mu},
\end{aligned}
$$

where the last inequality follows from the Cauchy-Schwarz inequality for the inner product $m(\cdot, \cdot),(2.8)$, and (6.4). We then set $v=e_{N}^{u, j}, q=e_{N}^{p, j}$ in (6.16), (6.17) and subtract the second from the first equation such that

$$
\begin{aligned}
\frac{1}{\Delta t} m\left(e_{N}^{u, j}-e_{N}^{u, j-1}, e_{N}^{u, j}\right)+\left\|e_{N}^{u, j}\right\|_{X, \mu}^{2} & =r_{N}^{1, j}\left(e_{N}^{u, j}\right)-r_{N}^{2, j}\left(e_{N}^{p, j}\right) \\
& \leq\left\|r_{N}^{1, j}\right\|_{X^{\prime}}\left\|e_{N}^{u, j}\right\|_{X}+\left\|r_{N}^{2, j}\right\|_{Y^{\prime}}\left\|e_{N}^{p, j}\right\|_{Y} .
\end{aligned}
$$

Applying now (6.18) and (2.10) yields

$$
\begin{aligned}
\frac{1}{\Delta t} m\left(e_{N}^{u, j}-e_{N}^{u, j-1}, e_{N}^{u, j}\right)+\left\|e_{N}^{u, j}\right\|_{X, \mu}^{2} & \\
\leq \frac{1}{\beta_{\mathrm{Br}}}\left\|r_{N}^{1, j}\right\|_{X^{\prime}}\left\|r_{N}^{2, j}\right\|_{Y^{\prime}}+\left(\left\|r_{N}^{1, j}\right\|_{X^{\prime}}\right. & \left.+\frac{\gamma_{a}}{\beta_{\mathrm{Br}}}\left\|r_{N}^{2, j}\right\|_{Y^{\prime}}\right) \frac{\left\|e_{N}^{u, j}\right\|_{X, \mu}}{\sqrt{\alpha_{a}}} \\
& +\frac{1}{\Delta t} \frac{\sqrt{\gamma_{m}}}{\beta_{\mathrm{Br}}}\left\|r_{N}^{2, j}\right\|_{Y^{\prime}}\left\|e_{N}^{u, j}-e_{N}^{u, j-1}\right\|_{\mu},
\end{aligned}
$$

which can be further bounded from Young's inequality by

$$
\begin{aligned}
\leq \frac{1}{\beta_{\mathrm{Br}}}\left\|r_{N}^{1, j}\right\|_{X^{\prime}}\left\|r_{N}^{2, j}\right\|_{Y^{\prime}}+\frac{1}{2 \alpha_{a}}\left(\left\|r_{N}^{1, j}\right\|_{X^{\prime}}+\frac{\gamma_{a}}{\beta_{\mathrm{Br}}}\left\|r_{N}^{2, j}\right\|_{Y^{\prime}}\right)^{2}+\frac{1}{2}\left\|e_{N}^{u, j}\right\|_{X, \mu}^{2} \\
+\frac{1}{2 \Delta t} \frac{\gamma_{m}}{\beta_{\mathrm{Br}}^{2}}\left\|r_{N}^{2, j}\right\|_{Y^{\prime}}^{2}+\frac{1}{2 \Delta t}\left\|e_{N}^{u, j}-e_{N}^{u, j-1}\right\|_{\mu}^{2} .
\end{aligned}
$$

Rearranging terms, the inequality now reads

$$
\begin{aligned}
& \frac{1}{\Delta t}\left(\left\|e_{N}^{u, j}\right\|_{\mu}^{2}-\left\|e_{N}^{u, j-1}\right\|_{\mu}^{2}\right)+\left\|e_{N}^{u, j}\right\|_{X, \mu}^{2} \\
& \quad \leq \frac{\left\|r_{N}^{1, j}\right\|_{X^{\prime}}^{2}}{\alpha_{a}}+\frac{2}{\beta_{\mathrm{Br}}}\left(1+\frac{\gamma_{a}}{\alpha_{a}}\right)\left\|r_{N}^{1, j}\right\|_{X^{\prime}}\left\|r_{N}^{2, j}\right\|_{Y^{\prime}}+\left(\frac{\gamma_{m}}{\Delta t}+\frac{\gamma_{a}^{2}}{\alpha_{a}}\right) \frac{\left\|r_{N}^{2, j}\right\|_{Y^{\prime}}^{2}}{\beta_{\mathrm{Br}}^{2}},
\end{aligned}
$$

and the result follows from applying the sum $\sum_{j=1}^{k}, e_{N}^{u, 0}=0$, and (2.29), (2.30), (6.13). 
Again, in the special case of a symmetric problem, the error bounds given in Proposition 6.4.1 can be improved. We may then derive the following result.

Proposition 6.4.2. Let $a(\cdot, \cdot ; \mu)$ be symmetric for all $\mu \in \mathcal{D}$. For any given $\mu \in \mathcal{D}, N \in$ $\mathbb{N}_{\max }, k \in \mathbb{K}$, and $\alpha_{a}^{\mathrm{LB}}(\mu), \gamma_{a}^{\mathrm{UB}}(\mu), \beta_{\mathrm{Br}}^{\mathrm{LB}}(\mu), \gamma_{m}^{\mathrm{UB}}(\mu)$ satisfying (2.29), (2.30), and (6.13), we define

$$
\begin{aligned}
\Delta_{N}^{\mathrm{sym}, k}(\mu) \equiv\left[\Delta t \sum_{j=1}^{k} \frac{\left\|r_{N}^{1, j}(\cdot ; \mu)\right\|_{X^{\prime}}^{2}}{\alpha_{a}^{\mathrm{LB}}(\mu)}+\frac{2}{\beta_{\mathrm{Br}}^{\mathrm{LB}}(\mu)}\left(1+\sqrt{\frac{\gamma_{a}^{\mathrm{UB}}(\mu)}{\alpha_{a}^{\mathrm{LB}}(\mu)}}\right)\left\|r_{N}^{1, j}(\cdot ; \mu)\right\|_{X^{\prime}}\left\|r_{N}^{2, j}(\cdot ; \mu)\right\|_{Y^{\prime}}\right. \\
\left.+\left(\frac{\gamma_{m}^{\mathrm{UB}}(\mu)}{\Delta t}+\gamma_{a}^{\mathrm{UB}}(\mu)\right) \frac{\left\|r_{N}^{2, j}(\cdot ; \mu)\right\|_{Y^{\prime}}^{2}}{\left(\beta_{\mathrm{Br}}^{\mathrm{LB}}(\mu)\right)^{2}}\right]^{1 / 2} \cdot(6.19)
\end{aligned}
$$

Then, $\Delta_{N}^{\mathrm{sym}, k}(\mu)$ represents an upper bound for the error $e_{N}^{u}(\mu)$ measured in the "spatiotemporal" energy norm (6.12),

$$
\left\|e_{N}^{u}(\mu)\right\|_{\ell^{2}(0, k ; X)} \leq \Delta_{N}^{\mathrm{sym}, k}(\mu), \quad \forall k \in \mathbb{K}, \mu \in \mathcal{D}, N \in \mathbb{N}_{\max } .
$$

Proof. Following the lines of the previous proof, we may now apply the Cauchy-Schwarz inequality for the inner product $a(\cdot, \cdot)$ to obtain

$$
\beta_{\mathrm{Br}}\left\|e_{N}^{p, j}\right\|_{Y} \leq\left\|r_{N}^{1, j}\right\|_{X^{\prime}}+\sqrt{\gamma_{a}}\left\|e_{N}^{u, j}\right\|_{X, \mu}+\frac{\sqrt{\gamma_{m}}}{\Delta t}\left\|e_{N}^{u, j}-e_{N}^{u, j-1}\right\|_{\mu}
$$

instead of (6.18). Proceeding as before, this yields

$$
\begin{aligned}
\frac{1}{\Delta t} m\left(e_{N}^{u, j}-e_{N}^{u, j-1}, e_{N}^{u, j}\right)+\left\|e_{N}^{u, j}\right\|_{X, \mu}^{2} & \\
\leq \frac{1}{\beta_{\mathrm{Br}}}\left\|r_{N}^{1, j}\right\|_{X^{\prime}}\left\|r_{N}^{2, j}\right\|_{Y^{\prime}}+\left(\frac{\left\|r_{N}^{1, j}\right\|_{X^{\prime}}}{\sqrt{\alpha_{a}}}+\right. & \left.\frac{\sqrt{\gamma_{a}}}{\beta_{\mathrm{Br}}}\left\|r_{N}^{2, j}\right\|_{Y^{\prime}}\right)\left\|e_{N}^{u, j}\right\|_{X, \mu} \\
& \quad+\frac{1}{\Delta t} \frac{\sqrt{\gamma_{m}}}{\beta_{\mathrm{Br}}}\left\|r_{N}^{2, j}\right\|_{Y^{\prime}}\left\|e_{N}^{u, j}-e_{N}^{u, j-1}\right\|_{\mu},
\end{aligned}
$$

and the statement again follows from applying Young's inequality, the sum $\sum_{j=1}^{k}, e_{N}^{u, 0}=0$, and (2.29), (2.30), (6.13).

\subsection{2 $\varepsilon>0$}

We here derive rigorous upper bounds for the error $e_{N}^{\varepsilon}(\mu)$ measured in the "spatio-temporal" energy norm

$$
\left\|\left(v^{j}, q^{j}\right)_{j \in \mathbb{K}}\right\|_{\ell^{2}(0, k ; Z)} \equiv\left(\left\|v^{k}\right\|_{\mu}^{2}+\Delta t \sum_{j=1}^{k}\left\|v^{j}\right\|_{X, \mu}^{2}+\varepsilon\left\|q^{j}\right\|_{Y, \mu}^{2}\right)^{1 / 2}
$$

where $\left(v^{j}, q^{j}\right)_{j \in \mathbb{K}} \subseteq Z, k \in \mathbb{K}$.

In addition to the dual norms of the residuals (6.10) and (6.11), we again also rely on (Online-)efficient lower (and upper) bounds (4.29) to the truth coercivity constants (2.10) and (4.12) to formulate our reduced basis a posteriori error bounds.

We can then formulate the following result. 
Proposition 6.4.3. For any given $\mu \in \mathcal{D}, N \in \mathbb{N}_{\max }, k \in \mathbb{K}$, and $\alpha_{a}^{\mathrm{LB}}(\mu), \alpha_{c}^{\mathrm{LB}}(\mu)$ satisfying (4.29), we define

$$
\Delta_{N}^{\varepsilon, k}(\mu) \equiv\left(\Delta t \sum_{j=1}^{k} \frac{\left\|r_{N}^{1, j}(\cdot ; \mu)\right\|_{X^{\prime}}^{2}}{\alpha_{a}^{\mathrm{LB}}(\mu)}+\frac{\left\|r_{N}^{2, j}(\cdot ; \mu)\right\|_{Y^{\prime}}^{2}}{\varepsilon \alpha_{c}^{\mathrm{LB}}(\mu)}\right)^{1 / 2}
$$

Then, $\Delta_{N}^{\varepsilon, k}(\mu)$ represents an upper bound for the error $e_{N}^{\varepsilon}(\mu)$ measured in the "spatiotemporal" energy norm (6.21),

$$
\left\|e_{N}^{\varepsilon}(\mu)\right\|_{\ell^{2}(0, k ; Z)} \leq \Delta_{N}^{\varepsilon, k}(\mu), \quad \forall k \in \mathbb{K}, \mu \in \mathcal{D}, N \in \mathbb{N}_{\max } .
$$

Proof. Let $\mu$ be any parameter in $\mathcal{D}, N \in \mathbb{N}_{\max }$, and $k \in \mathbb{K}$. For clarity of exposition, we suppress the argument $\mu$ in this proof.

Take any $1 \leq j \leq k$. From (6.10), (6.11), and (6.6), the errors $e_{N}^{u, j} \in X$ and $e_{N}^{p, j} \in Y$ satisfy the equations

$$
\begin{aligned}
& \frac{1}{\Delta t} m\left(e_{N}^{u, j}-e_{N}^{u, j-1}, v\right)+a\left(e_{N}^{u, j}, v\right)+b\left(v, e_{N}^{p, j}\right)=r_{N}^{1, j}(v), \quad \forall v \in X, \\
& b\left(e_{N}^{u, j}, q\right)-\varepsilon c\left(e_{N}^{p, j}, q\right)=r_{N}^{2, j}(q), \quad \forall q \in Y .
\end{aligned}
$$

Setting here $v=e_{N}^{u, j}, q=e_{N}^{p, j}$ and subtracting the second from the first equation, we obtain

$$
\begin{aligned}
\frac{1}{\Delta t} m\left(e_{N}^{u, j}-e_{N}^{u, j-1}, e_{N}^{u, j}\right)+\left\|e_{N}^{u, j}\right\|_{X, \mu}^{2}+\varepsilon\left\|e_{N}^{p, j}\right\|_{Y, \mu}^{2}=r_{N}^{1, j}\left(e_{N}^{u, j}\right)-r_{N}^{2, j}\left(e_{N}^{p, j}\right) \\
\leq\left\|r_{N}^{1, j}\right\|_{X^{\prime}}\left\|e_{N}^{u, j}\right\|_{X}+\left\|r_{N}^{2, j}\right\|_{Y^{\prime}}\left\|e_{N}^{p, j}\right\|_{Y} .
\end{aligned}
$$

On the right-hand side, we now use (2.10), (4.12), and Young's inequality so that

$$
\begin{aligned}
\left\|r_{N}^{1, j}\right\|_{X^{\prime}}\left\|e_{N}^{u, j}\right\|_{X}+\left\|r_{N}^{2, j}\right\|_{Y^{\prime}}\left\|e_{N}^{p, j}\right\|_{Y} & \leq \frac{\left\|r_{N}^{1, j}\right\|_{X^{\prime}}}{\sqrt{\alpha_{a}}}\left\|e_{N}^{u, j}\right\|_{X, \mu}+\frac{\left\|r_{N}^{2, j}\right\|_{Y^{\prime}}}{\sqrt{\alpha_{c}}}\left\|e_{N}^{p, j}\right\|_{Y, \mu} \\
& \leq \frac{1}{2}\left(\frac{\left\|r_{N}^{1, j}\right\|_{X^{\prime}}^{2}}{\alpha_{a}}+\left\|e_{N}^{u, j}\right\|_{X, \mu}^{2}+\frac{\left\|r_{N}^{2, j}\right\|_{Y^{\prime}}^{2}}{\varepsilon \alpha_{c}}+\varepsilon\left\|e_{N}^{p, j}\right\|_{Y, \mu}^{2}\right)
\end{aligned}
$$

on the left-hand side, we use the Cauchy-Schwarz inequality for the inner product $m(\cdot, \cdot)$ followed by Young's inequality so that

$$
m\left(e_{N}^{u, j}-e_{N}^{u, j-1}, e_{N}^{u, j}\right) \geq\left\|e_{N}^{u, j}\right\|_{\mu}^{2}-\left\|e_{N}^{u, j-1}\right\|_{\mu}\left\|e_{N}^{u, j}\right\|_{\mu} \geq \frac{1}{2}\left(\left\|e_{N}^{u, j}\right\|_{\mu}^{2}-\left\|e_{N}^{u, j-1}\right\|_{\mu}^{2}\right) .
$$

Rearranging terms, the inequality (6.24) finally reads

$$
\frac{1}{\Delta t}\left(\left\|e_{N}^{u, j}\right\|_{\mu}^{2}-\left\|e_{N}^{u, j-1}\right\|_{\mu}^{2}\right)+\left\|e_{N}^{u, j}\right\|_{X, \mu}^{2}+\varepsilon\left\|e_{N}^{p, j}\right\|_{Y, \mu}^{2} \leq \frac{\left\|r_{N}^{1, j}\right\|_{X^{\prime}}^{2}}{\alpha_{a}}+\frac{\left\|r_{N}^{2, j}\right\|_{Y^{\prime}}^{2}}{\varepsilon \alpha_{c}}
$$

and the statement follows from applying the sum $\sum_{j=1}^{k}, e_{N}^{u, 0}=0$, and (4.29). 


\subsection{Offline-Online Computational Procedure}

Again, the basic strategy lies in the $\mu$-affine dependence of the involved operators. All $\mu$ independent quantities in (2.7) can be formed and stored within a computationally expensive Offline stage, which is performed only once and the cost of which depends on the large finite element dimension $\mathcal{N}$. For any given parameter $\mu \in \mathcal{D}$, the reduced basis approximation $\left(u_{N}^{\varepsilon, k}(\mu), p_{N}^{\varepsilon, k}(\mu)\right), k \in \mathbb{K}$, is then computed within a highly efficient Online stage; the cost does not depend on $\mathcal{N}$ but only on the much smaller dimension of the reduced basis approximation space. The computation of the a posteriori error bounds again consists of two components: the calculation of the residual dual norms $\left\|r_{N}^{1, k}(\cdot ; \mu)\right\|_{X^{\prime}},\left\|r_{N}^{2, k}(\cdot ; \mu)\right\|_{Y^{\prime}}, k \in \mathbb{K}$, and the calculation of the required lower and upper bounds (2.29), (2.30), (6.13) and (4.29), respectively, to the involved constants. Except for the additional mass term, the exact computational procedure does not significantly differ from the stationary case; therefore, we do not give further explanations at this point but refer the reader to $22.6, \S 4.6$, and [37].

\subsection{Construction of Reduced Basis Approximation Spaces}

We now turn to the construction of the reduced basis approximation spaces $X_{N}, Y_{N}, N \in$ $\mathbb{N}_{\max }$. According to the reduced basis idea (see $\S 2.5$ ), the low-dimensional spaces $X_{N}, Y_{N}$ are again constructed by exploiting the parametric structure of the problem: Basis functions are essentially given by truth solutions associated with several chosen parameter snapshots. To provide accurate approximations $\left(u_{N}^{\varepsilon, k}(\mu), p_{N}^{\varepsilon, k}(\mu)\right)$ for $\left(u^{\varepsilon, k}(\mu), p^{\varepsilon, k}(\mu)\right), k \in \mathbb{K}$, for any parameter query, $X_{N}$ and $Y_{N}$ here not only have to appropriately represent the manifold $\mathcal{M}$ induced by the parametric dependence (see $\S 2.5$ ), but also need to capture the causality associated with evolution in time. Again, keeping computational cost to a minimum, we aim to achieve this with as few basis functions as possible.

The POD greedy procedure represents an adaptive sampling process for parabolic problems that properly accounts for temporal and parametric causality: It combines the proper orthogonal decomposition (POD) method in $k$ (see $[68,69]$ ) and also $§ 1.2)$ with the greedy procedure in $\mu$ (see $\S 2.7,[10,15]$ ). To begin with, we briefly recall the optimality property of the POD as described in $[68,69]$. For a given finite set $\mathcal{X}_{\mathcal{I}} \equiv\left\{\chi_{1}, \ldots, \chi_{\mathcal{I}}\right\} \subseteq X$ and $M_{X} \leq \operatorname{dim}\left(\operatorname{span}\left(\mathcal{X}_{\mathcal{I}}\right)\right)$, the POD basis of rank $M_{X}$ consists of $M_{X}(\cdot, \cdot)_{X}$-orthonormal basis functions that approximate $\mathcal{X}_{\mathcal{I}}$ best in the sense that

$$
\operatorname{span}\left(\operatorname{POD}_{X}\left(\mathcal{X}_{\mathcal{I}}, M_{X}\right)\right)=\arg \inf _{\substack{\mathcal{X} \subseteq \operatorname{span}\left(\mathcal{X}_{\mathcal{I}}\right) \\ \operatorname{dim}(\mathcal{X})=M_{X}}}\left(\frac{1}{\mathcal{I}} \sum_{i=1}^{\mathcal{I}} \inf _{\chi \in \mathcal{X}}\left\|\chi_{i}-\chi\right\|_{X}^{2}\right)^{1 / 2} ;
$$

analogously, we denote by $\operatorname{POD}_{Y}\left(\mathcal{Y}_{\mathcal{I}}, M_{Y}\right)$ the POD basis of rank $M_{Y}$ for a finite set $\mathcal{Y}_{\mathcal{I}} \subseteq Y$, $M_{Y} \leq \operatorname{dim}\left(\operatorname{span}\left(\mathcal{Y}_{\mathcal{I}}\right)\right)$. Assuming that we are given a current pair $\left(X_{N-1}, Y_{N-1}\right)$ of reduced basis approximation spaces, the POD greedy algorithm now proceeds as follows: In compliance with the greedy approach, it detects the parameter $\mu_{N}$ for which the (Online-)efficient reduced basis error bound attains its maximum over an exhaustive sample $\Sigma \subset \mathcal{D}$. For a prescribed $\Delta N \in \mathbb{K}$, we then compute the POD bases of rank $\Delta N$ associated with the truth solutions $u^{\varepsilon, k}\left(\mu_{N}\right)$ and $p^{\varepsilon, k}\left(\mu_{N}\right), k \in \mathbb{K}$; more specifically, we compute $\operatorname{POD}_{X}\left(E^{u}, \Delta N\right)$ and 
$\operatorname{POD}_{Y}\left(E^{p}, \Delta N\right)$ for

$$
\begin{aligned}
E^{u} & \equiv\left\{u^{\varepsilon, k}\left(\mu_{N}\right)-\Pi_{X_{N-1}} u^{\varepsilon, k}\left(\mu_{N}\right) \mid k \in \mathbb{K}\right\}, \\
E^{p} & \equiv\left\{p^{\varepsilon, k}\left(\mu_{N}\right)-\Pi_{Y_{N-1}} p^{\varepsilon, k}\left(\mu_{N}\right) \mid k \in \mathbb{K}\right\},
\end{aligned}
$$

where $\Pi_{X_{N-1}}$ and $\Pi_{Y_{N-1}}$ refer to the $(\cdot, \cdot)_{X^{-}}$and $(\cdot, \cdot)_{Y}$-orthogonal projections on the current reduced basis approximation spaces $X_{N-1}$ and $Y_{N-1}$, respectively. Finally, the $\Delta N$ POD basis functions are appended to $X_{N-1}$ and $Y_{N-1}$, and we obtain a subsequent pair $\left(X_{N}, Y_{N}\right)$. As before, this process is then repeated until a prescribed error tolerance is satisfied. We refer the reader to $[37,46,47]$ for a detailed discussion of the POD greedy procedure, and to $[63,64]$ for an application to the Boussinesq and Fokker-Planck equations.

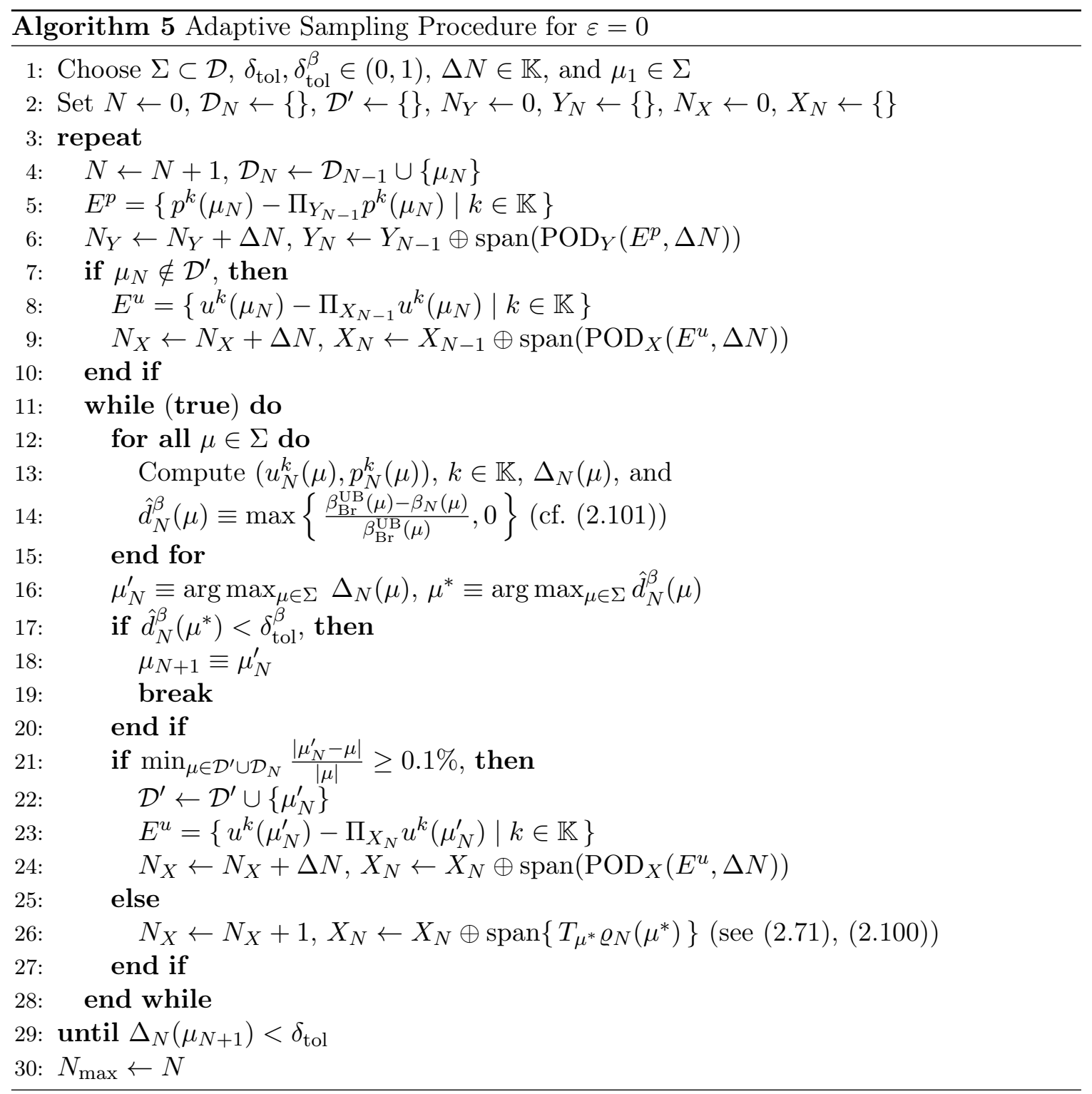


For our saddle point problems, we now couple the above procedure with stabilization techniques developed in $\S 2.7$. In $\S 3.3 .3$ and $\S 5.3 .2$, best convergence results were achieved by Algorithm 3 where we aim to stabilize through an enrichment of the primal reduced basis approximation space with additional truth solutions. According to these observations, we here apply the sampling procedures presented in Algorithm 5 and Algorithm 6. In case of $\varepsilon=0$, we again use the distance $d_{N}^{\beta}(\mu)$ (see (2.101)) of the inf-sup constants $\beta_{N}(\mu)$ to the truth Brezzi inf-sup constants $\beta_{\mathrm{Br}}(\mu)$ as an indicator whether a current pair of reduced basis approximation spaces needs to be stabilized; the exact procedure is given in Algorithm 5 . In case of $\varepsilon>0$, numerical results in $\S 5.3 .2$ showed that the inf-sup constants $\beta_{N}(\mu)$ may not be appropriate indicators for an ill-conditioned system but an adaptive sampling process should be based rather on the condition number $\kappa_{N}^{\varepsilon}(\mu)$ (see (5.6)); Algorithm 6 now presents a possibility how this could be realized.

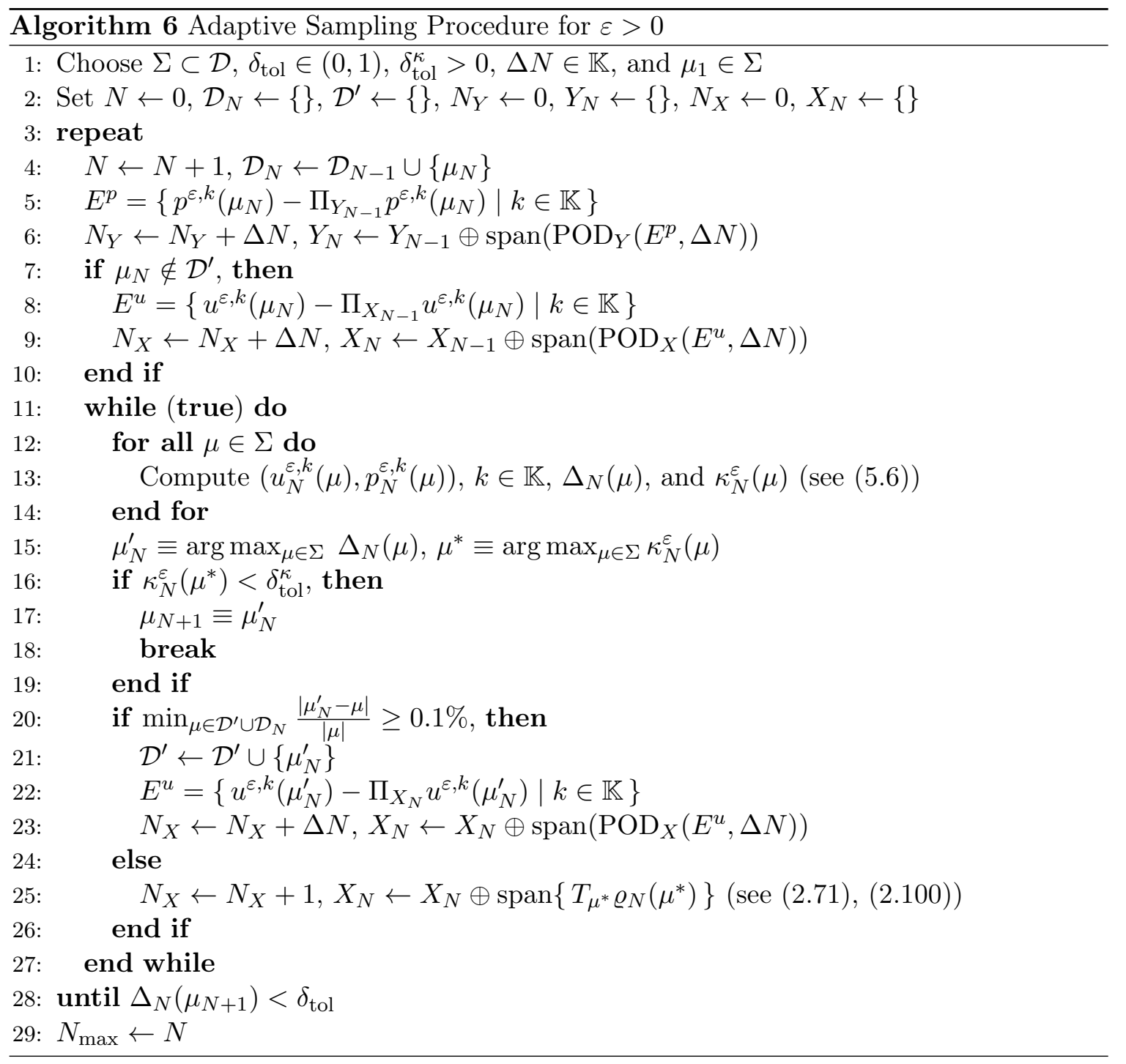




\subsection{Model Problem}

We again consider a Stokes flow in a two-dimensional microchannel with an obstacle as introduced in $\S 3.2$; evolution in time is now induced by a time-dependent velocity profile on the inflow boundary.

Let $\mu$ be any parameter in $\mathcal{D}$. For the physical domain $\tilde{\Omega}$ and a given time interval $[0, T]$, $T>0$, we now seek to find the (inhomogeneous) velocity $\tilde{u}_{\mathrm{e}, \text { inh }}: \tilde{\Omega} \times(0, T) \rightarrow \mathbb{R}^{2}$ and the pressure $\tilde{p}_{\mathrm{e}}: \tilde{\Omega} \times(0, T) \rightarrow \mathbb{R}$ satisfying

$$
\begin{aligned}
\frac{\partial \tilde{u}_{\mathrm{e}, \mathrm{inh}}}{\partial t}-\tilde{\Delta} \tilde{u}_{\mathrm{e}, \mathrm{inh}}+\tilde{\nabla} \tilde{p}_{\mathrm{e}}=0 & \text { in } \tilde{\Omega} \times(0, T), \\
\tilde{\nabla} \cdot \tilde{u}_{\mathrm{e}, \mathrm{inh}}=0 & \text { in } \tilde{\Omega} \times(0, T),
\end{aligned}
$$

subject to initial conditions $\tilde{u}_{\mathrm{e}, \mathrm{inh}}(\cdot, 0)=0$ and with boundary conditions

$$
\begin{gathered}
\tilde{u}_{\mathrm{e}, \mathrm{inh}}(\tilde{x}, t)=H(t) h(\tilde{x}) \quad \text { on } \Gamma_{\mathrm{in}} \times(0, T), \quad \tilde{u}_{\mathrm{e}, \text { inh }}=0 \quad \text { on } \tilde{\Gamma}_{0} \times(0, T), \\
\frac{\partial \tilde{u}_{\mathrm{e}, \text { inh }}}{\partial \tilde{n}}=\tilde{p}_{\mathrm{e}} \tilde{n} \quad \text { on } \Gamma_{\text {out }} \times(0, T) ;
\end{gathered}
$$

here, $\tilde{\Delta}$ and $\tilde{\nabla}$ again denote the Laplacian and gradient operator over the physical domain $\tilde{\Omega}$ (see Appendix A), $\tilde{n}$ is the unit outward normal, $h: \mathbb{R}^{2} \rightarrow \mathbb{R}^{2}$ is given as in $\S 3.2 .1$ by $h(x) \equiv\left(4 x_{2}\left(1-x_{2}\right), 0\right)$ for all $x=\left(x_{1}, x_{2}\right) \in \mathbb{R}^{2}$, and we choose $H:[0, T] \rightarrow \mathbb{R}$ with $H(t) \equiv t(\sin (2 \pi t)+1)$ for all $t \in[0, T]$. According to the setting introduced in $\S 5.2$, we also consider the following perturbation of the problem (6.25)-(6.27): For a sufficiently small $\varepsilon>0$, we again introduce a penalty term to the continuity equation (6.26) such that

$$
\tilde{\nabla} \cdot \tilde{u}_{\mathrm{e}, \mathrm{inh}}^{\varepsilon}=-\varepsilon p_{\mathrm{e}}^{\varepsilon} \quad \text { in } \tilde{\Omega} \times(0, T) .
$$

We now follow the steps discussed in $§ 3.2 .2, \S 3.2 .3$, and $\S 5.2$ : We choose the lifting function $\tilde{u}_{\mathrm{L}}^{H} \equiv H \tilde{u}_{\mathrm{L}}$ where $\tilde{u}_{\mathrm{L}}$ is defined as in (3.4), and transform the problem statement for the homogeneous velocity $\tilde{u}_{\mathrm{e}}^{\varepsilon} \equiv \tilde{u}_{\mathrm{e}, \text { inh }}^{\varepsilon}-\tilde{u}_{\mathrm{L}}^{H}$ to an equivalent problem posed over the reference domain $\Omega$. Furthermore, as required for the time-discrete setting introduced in $\S 6.2$, we divide the time interval $[0, T]$ into $K$ subintervals of equal length $\Delta t \equiv T / K$, and consider a backward Euler method for time integration. The problems (6.25)-(6.27) and (6.25), (6.28), (6.27) may thus be written as a parametrized saddle point problem of the form (6.3). Here, for any $\mu \in \mathcal{D}$, the bilinear forms $a(\cdot, \cdot ; \mu), b(\cdot, \cdot ; \mu)$, and $c(\cdot, \cdot ; \mu)$ are given as in (3.8), (3.9), and (5.5), respectively; accordingly, the bilinear form $m(\cdot, \cdot ; \mu): X_{\mathrm{e}} \times X_{\mathrm{e}} \rightarrow \mathbb{R}$ represents the $L^{2}$-inner product for vector functions over the physical domain $\tilde{\Omega}$ formulated on the reference domain $\Omega$ (see $\S 3.2 .3$ ),

$$
m(u, v ; \mu)=\sum_{s=1}^{S} \frac{1}{\left|\operatorname{det}\left(A^{s}(\mu)\right)\right|} \int_{\Omega^{s}} u \cdot v d x, \quad \forall u, v \in X_{\mathrm{e}},
$$

and the linear functionals $f(\cdot ; \mu)$ and $g(\cdot ; \mu)$ are given by

$$
\begin{aligned}
& f(v, t ; \mu)=f(v, t)=-H^{\prime}(t) \int_{\Omega_{\mathrm{L}}} u_{\mathrm{L}} \cdot v d x-H(t) \int_{\Omega_{\mathrm{L}}} \frac{\partial u_{\mathrm{L} i}}{\partial x_{j}} \frac{\partial v_{i}}{\partial x_{j}} d x, \quad \forall v \in X_{\mathrm{e}}, t \in[0, T], \\
& g(q, t ; \mu)=g(q, t)=H(t) \int_{\Omega_{\mathrm{L}}} q \frac{\partial u_{\mathrm{L} i}}{\partial x_{i}} d x, \quad \forall q \in Y_{\mathrm{e}}, t \in[0, T] .
\end{aligned}
$$


We recall that the bilinear forms $a(\cdot, \cdot ; \mu), b(\cdot, \cdot ; \mu)$, and $c(\cdot, \cdot ; \mu)$ then satisfy the assumptions (2.1)-(2.4), (4.1), and (4.2). For all $\mu \in \mathcal{D}, m(\cdot, \cdot ; \mu)$ defines an inner product on $X_{\mathrm{e}}$ such that (6.2) holds true; moreover, there exists a constant $C_{4}^{\mathrm{e}}(\mu)>0$ from the Poincare inequality (see, e.g., [89]) such that

$$
m(v, v ; \mu) \leq C_{4}^{\mathrm{e}}(\mu) a(v, v ; \mu), \quad \forall v \in X_{\mathrm{e}}, \quad \forall \mu \in \mathcal{D},
$$

and thus (6.1) is satisfied with

$$
\gamma_{m}^{\mathrm{e}}(\mu) \equiv \sup _{u \in X_{\mathrm{e}}} \sup _{v \in X_{\mathrm{e}}} \frac{m(u, v ; \mu)}{\|u\|_{X_{\mathrm{e}}}\|v\|_{X_{\mathrm{e}}}} \leq C_{4}^{\mathrm{e}}(\mu) \gamma_{a}^{\mathrm{e}}(\mu)<\infty, \quad \forall \mu \in \mathcal{D} .
$$

We particularly have $Q_{a}=10, Q_{b}=6, Q_{f}=Q_{g}=1$, and $Q_{m}=Q_{c}=5$ in the respective $\mu$-affine expansions (2.7). Choosing again the truth approximation spaces $X$ and $Y$ as the standard conforming $\mathbb{P}_{2}-\mathbb{P}_{1}$ Taylor-Hood finite element approximation subspaces [100] over the regular triangulation $\mathcal{T}_{\Omega}$, we ensure that also (2.11) is satisfied and therefore recover the situation described in $\S 6.2 .2$.

\subsection{Numerical Results}

We now apply the reduced basis methodology developed in $\S 6.3-\S 6.6$ to our model problem. We set $T=1$ and consider a constant time step size $\Delta t$ corresponding to $K=100$ time levels. The truth discretization is based on a fine mesh with a total of $\mathcal{N}=72,076$ velocity and pressure degrees of freedom. In this section, all numerical results are attained using the open source software rbOOmit [65], an implementation of the reduced basis framework within the $\mathrm{C}++$ parallel finite element library libMesh [62].

\subsection{1 $\varepsilon=0$}

We first turn to the coercivity, continuity, and inf-sup constants required for our reduced basis procedure. We again obtain (Online-)efficient lower and upper bounds to $\alpha_{a}(\mu), \gamma_{a}(\mu)$, and $\beta_{\mathrm{Br}}(\mu)$ by using the SCM (see $\S 2.6 .2$ ) with the configurations specified in $\S 3.3 .4$. To estimate the continuity constants $\gamma_{m}(\mu)$, we apply the method for $M_{\alpha}=\infty$, an exhaustive sample $\Xi \subset \mathcal{D}$ of size $|\Xi|=4,225$, and $\delta_{\text {tol }}^{\mathrm{SCM}}=0.01$. We then obtain $K_{\max }=5$, and Fig. 6.1 presents the resulting accurate (Online-)efficient lower and upper bounds $\gamma_{m}^{\mathrm{LB}}(\mu)$ and $\gamma_{m}^{\mathrm{UB}}(\mu)$.

We now turn to the reduced basis approximation. To build our low-dimensional reduced basis approximation spaces $X_{N}, Y_{N}, N \in \mathbb{N}_{\max }$, we apply the POD greedy procedure described in Algorithm 5 (see §6.6). The sampling process is based on an exhaustive random sample $\Sigma \subset \mathcal{D}$ of size $|\Sigma|=4,900, \Delta N=2$, and $\delta_{\text {tol }}^{\beta}=0.1$; since our Stokes model problem is clearly symmetric, we here in particular use the relative reduced basis a posteriori error bound $\Delta_{N}(\mu) \equiv \Delta_{N}^{\text {sym, } K}(\mu) /\left\|\left(u_{N}^{j}(\mu)\right)_{j \in \mathbb{K}}\right\|_{\ell^{2}(0, K ; X)}$ (see (6.12), (6.19)). Figure 6.2 now shows the maximum error $\left\|e_{N}^{u}(\mu)\right\|_{\ell^{2}(0, K ; X)}$ (see (6.9)) in the reduced basis velocity approximations and associated error bounds $\Delta_{N}^{\text {sym, } K}(\mu)$ and $\Delta_{N}^{K}(\mu)$ (see (6.14)) as functions of the dimension $N_{Z}$; Figure 6.3 presents the maximum error $\left\|e_{N}^{u}(\mu)\right\|_{\ell^{2}(0, k ; X)}$ and associated error bounds $\Delta_{N}^{\mathrm{sym}, k}(\mu), \Delta_{N}^{k}(\mu)$ as functions of $k \in \mathbb{K}$ for several values of $N_{Z}$. First, we observe 

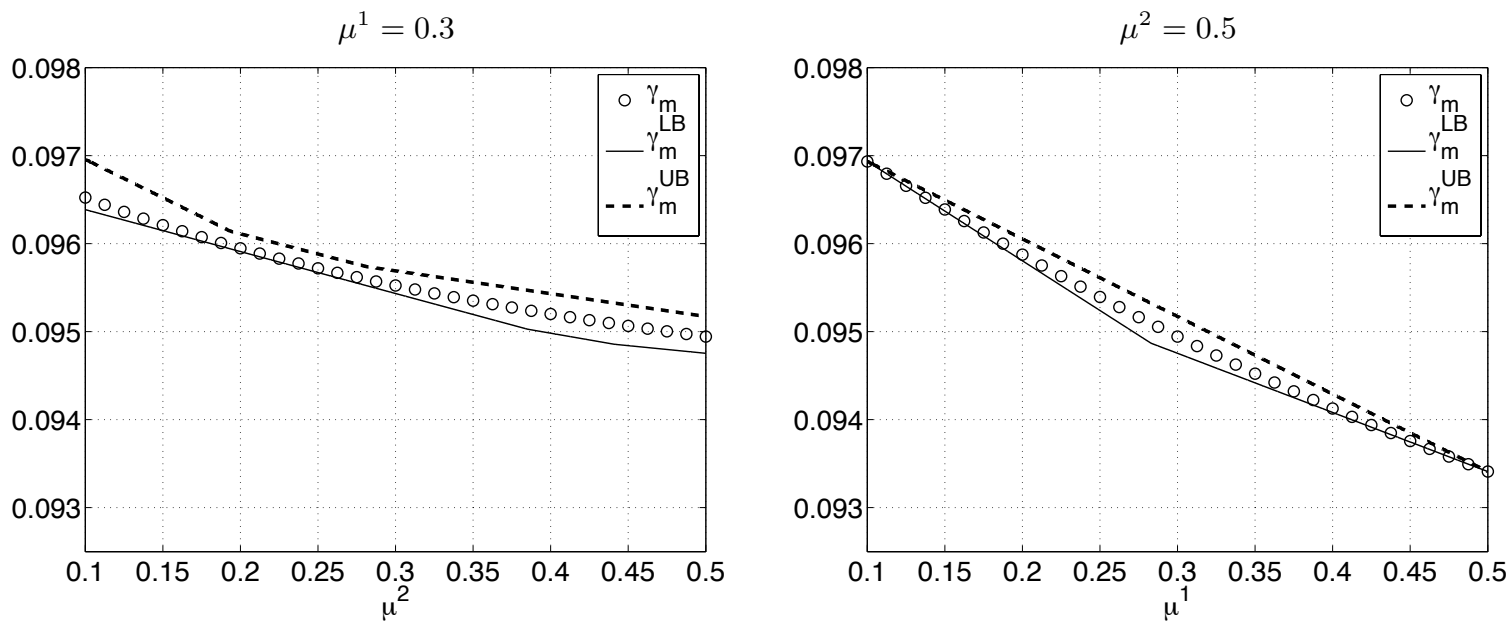

Figure 6.1.: SCM lower and upper bounds $\gamma_{m}^{\mathrm{LB}}(\mu)$ and $\gamma_{m}^{\mathrm{UB}}(\mu)$ for the continuity constants $\gamma_{m}(\mu)$ (see (6.4), (6.13)) where $|\Xi|=4,225, M_{\alpha}=\infty, \delta_{\text {tol }}^{\mathrm{SCM}}=0.01$, and $K_{\max }=5$.

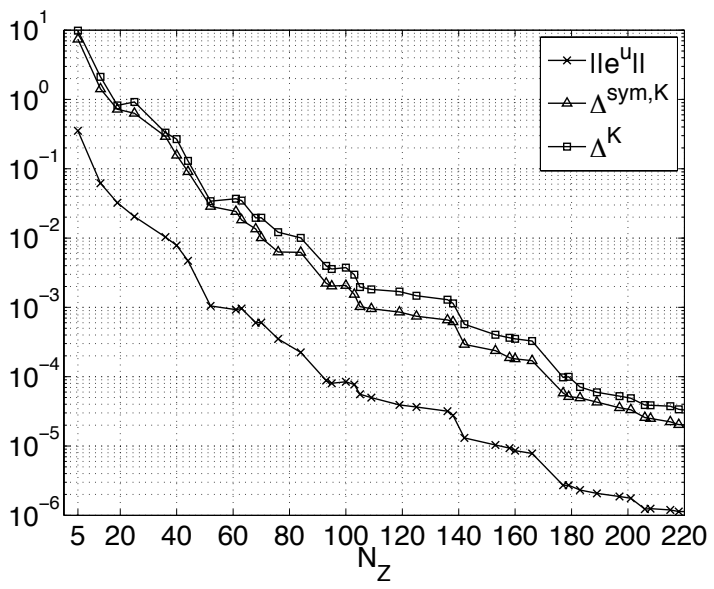

Figure 6.2.: Maximum error $\left\|e_{N}^{u}(\mu)\right\|_{\ell^{2}(0, K ; X)}$ (see (6.9), (6.12)) and maximum error bounds $\Delta_{N}^{\mathrm{sym}, K}(\mu)$ and $\Delta_{N}^{K}(\mu)$ (see (6.19) and (6.14)) normalized with respect to $\left\|\left(u^{j}(\mu)\right)_{j \in \mathbb{K}}\right\|_{\ell^{2}(0, K ; X)}$ shown as functions of $N_{Z}$; the maximum is taken over 25 parameter values. 

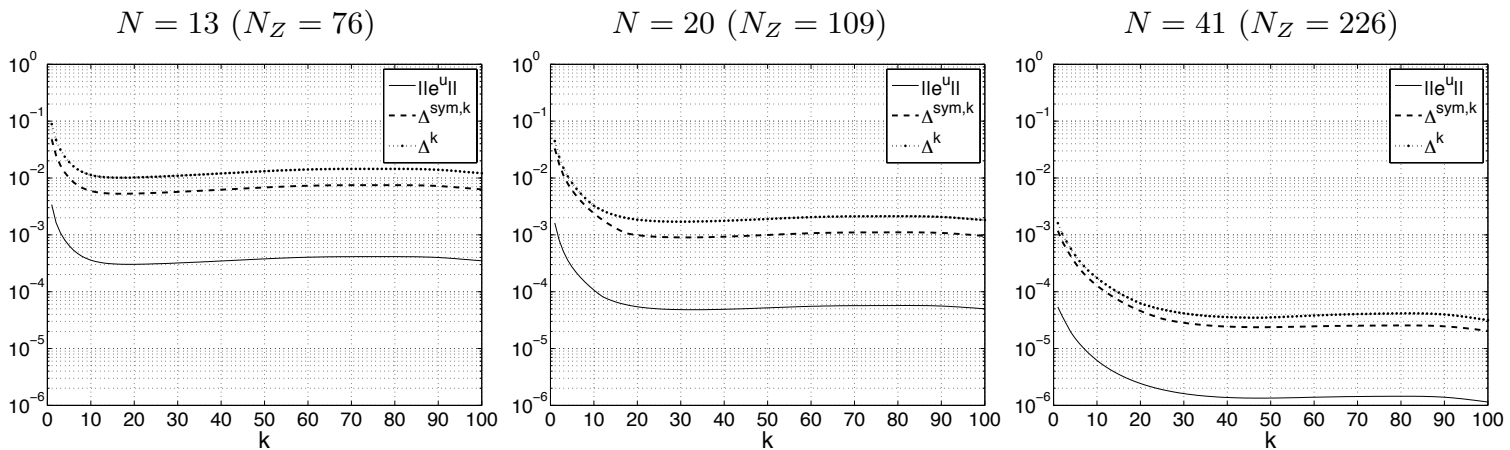

Figure 6.3.: Maximum error $\left\|e_{N}^{u}(\mu)\right\|_{\ell^{2}(0, k ; X)}$ (see (6.9), (6.12)) and maximum error bounds $\Delta_{N}^{\mathrm{sym}, k}(\mu)$ and $\Delta_{N}^{k}(\mu)$ (see (6.19) and (6.14)) normalized with respect to $\left\|\left(u^{j}(\mu)\right)_{j \in \mathbb{K}}\right\|_{\ell^{2}(0, k ; X)}$ shown as functions of $k \in \mathbb{K}$ for several values of $N$; the maximum is taken over 25 parameter values.

(a) Effectivities $\eta_{N}^{\mathrm{sym}, k}(\mu)$ associated with $\Delta_{N}^{\mathrm{sym}, k}(\mu)$

\begin{tabular}{c|c|c|c|c|c|c|c|c|c|c|c}
\hline$N$ & $N_{Z}$ & $k=10$ & $k=20$ & $k=30$ & $k=40$ & $k=50$ & $k=60$ & $k=70$ & $k=80$ & $k=90$ & $k=100$ \\
\hline 5 & 36 & 28.90 & 30.38 & 30.96 & 31.30 & 31.51 & 31.61 & 31.63 & 31.63 & 31.52 & 31.13 \\
10 & 63 & 30.41 & 31.35 & 31.82 & 32.20 & 32.35 & 32.37 & 32.29 & 32.16 & 32.01 & 31.91 \\
15 & 93 & 25.83 & 28.05 & 29.12 & 29.39 & 29.48 & 29.51 & 29.49 & 29.44 & 29.40 & 29.38 \\
20 & 109 & 23.31 & 24.25 & 26.10 & 26.79 & 27.08 & 27.23 & 27.22 & 27.08 & 27.00 & 27.21 \\
25 & 142 & 25.29 & 28.15 & 29.20 & 29.54 & 29.68 & 29.73 & 29.72 & 29.66 & 29.60 & 29.64 \\
30 & 177 & 26.28 & 26.05 & 27.18 & 28.77 & 30.06 & 30.58 & 30.72 & 30.70 & 30.59 & 30.60 \\
35 & 201 & 24.77 & 24.86 & 25.70 & 26.68 & 27.18 & 27.36 & 27.45 & 27.51 & 27.50 & 27.81 \\
40 & 222 & 24.18 & 23.96 & 24.04 & 24.19 & 24.65 & 25.03 & 25.37 & 25.51 & 25.50 & 25.54 \\
\hline
\end{tabular}

(b) Effectivities $\eta_{N}^{k}(\mu)$ associated with $\Delta_{N}^{k}(\mu)$

\begin{tabular}{c|c|c|c|c|c|c|c|c|c|c|c}
\hline$N$ & $N_{Z}$ & $k=10$ & $k=20$ & $k=30$ & $k=40$ & $k=50$ & $k=60$ & $k=70$ & $k=80$ & $k=90$ & $k=100$ \\
\hline 5 & 36 & 39.58 & 41.51 & 42.30 & 42.76 & 43.05 & 43.20 & 43.22 & 43.22 & 43.06 & 42.53 \\
10 & 63 & 39.90 & 41.66 & 42.46 & 42.82 & 43.08 & 43.21 & 43.16 & 43.00 & 42.91 & 42.90 \\
15 & 93 & 38.58 & 43.21 & 44.23 & 44.64 & 44.93 & 45.13 & 45.17 & 45.14 & 45.18 & 45.07 \\
20 & 109 & 32.59 & 34.10 & 35.30 & 37.11 & 37.61 & 37.60 & 37.34 & 37.17 & 36.88 & 37.19 \\
25 & 142 & 35.52 & 39.26 & 42.22 & 42.93 & 42.87 & 42.73 & 42.71 & 42.63 & 42.67 & 43.55 \\
30 & 177 & 34.31 & 34.27 & 34.61 & 36.41 & 37.23 & 37.31 & 38.27 & 39.67 & 40.42 & 40.64 \\
35 & 201 & 32.86 & 33.59 & 35.05 & 36.52 & 37.33 & 37.39 & 36.98 & 36.65 & 36.77 & 37.54 \\
40 & 222 & 33.67 & 33.76 & 34.45 & 35.25 & 35.59 & 35.42 & 35.01 & 34.78 & 34.87 & 35.17 \\
\hline
\end{tabular}

Table 6.1.: Maximum effectivities (a) $\eta_{N}^{\mathrm{sym}, k}(\mu) \equiv \Delta_{N}^{\mathrm{sym}, k}(\mu) /\left\|e_{N}^{u}(\mu)\right\|_{\ell^{2}(0, k ; X)}$ (see (6.20)) and (b) $\eta_{N}^{k}(\mu) \equiv \Delta_{N}^{k}(\mu) /\left\|e_{N}^{u}(\mu)\right\|_{\ell^{2}(0, k ; X)}$ (see (6.15)) for several values of $k \in \mathbb{K}$ and $N$; the maximum is taken over 25 parameter values. 
that the reduced basis error and error bounds are roughly uniform in time (see Fig. 6.3) and decrease rapidly as $N_{Z}$ increases (see Fig. 6.2). We obtain stable, rapidly convergent reduced basis approximations, and rigorous a posteriori error bounds that reflect the behavior of the error very accurately. Second, the error bounds are tight. To quantify this statement, we present in Table 6.1 maximum effectivities associated with $\Delta_{N}^{\mathrm{sym}, k}(\mu)$ and $\Delta_{N}^{k}(\mu)$ for several values of $k$ and $N$. We notice that their values remain more or less constant with $k$. Moreover, as in the stationary case (see §3.3.4), we again benefit from exploiting the symmetry of the problem: Effectivities range from 33 to 45 in case of $\Delta_{N}^{k}(\mu)$ (see Table 6.1(b)) and improve in case of $\Delta_{N}^{\mathrm{sym}, k}(\mu)$ by roughly 10 (see Table 6.1(a)). We emphasize at this point that the error bound formulations in (6.14) and (6.19) in fact suggest a growth in time. In practice, this behavior seems rather weak (see Table 6.1) but may be investigated in greater detail within future work.

We now discuss the Online computation times for the proposed method. For comparison, once the $\mu$-independent parts in (2.7) have been formed, direct computation of the truth approximation $\left(u^{k}(\mu), p^{k}(\mu)\right), k \in \mathbb{K}$, (i.e., assembly and solution of (6.6)) requires roughly 30 seconds on a $2.66 \mathrm{GHz}$ Intel Core 2 Duo processor. We initially take a total reduced basis dimension of $N_{Z}=226$. Once the database has been loaded, the Online calculation of $\left(u_{N}^{k}(\mu), p_{N}^{k}(\mu)\right), k \in \mathbb{K}$, (i.e., assembly and solution of $\left.(6.7)\right)$ and $\Delta_{N}^{\mathrm{sym}, k}(\mu), k \in \mathbb{K}$, for any new value of $\mu \in \mathcal{D}$ takes on average 27.97 and 80.76 milliseconds, respectively, which is in total roughly 270 times faster than direct computation of the truth approximation. Thus, even for this large value of $N_{Z}$, we obtain significant Online savings. In practice, however, we quite often need not take such a large value of $N_{Z}$ - our rigorous and inexpensive error bounds $\Delta_{N}^{\mathrm{sym}, k}(\mu), k \in \mathbb{K}$, allow us to choose the reduced basis dimension just large enough to obtain a desired accuracy. To achieve a prescribed accuracy of at least $1 \%$ (resp., 0.1\%) in the reduced basis approximations $u_{N}^{k}(\mu), k \in \mathbb{K}$, we need $N_{Z}=76$ (resp., $N_{Z}=109$ ) (see Fig. 6.2). Again, once the database has been loaded, the Online calculation of $\left(u_{N}^{k}(\mu), p_{N}^{k}(\mu)\right), k \in \mathbb{K}$, and $\Delta_{N}^{\text {sym }, k}(\mu), k \in \mathbb{K}$, for any new value of $\mu \in \mathcal{D}$ takes on average 4.41 (resp., 7.62 ) and 24.47 (resp., 33.75) milliseconds, respectively, which is in total roughly 1,000 times (resp., 700 times) faster than direct computation of the truth approximation.

\subsection{2 $\varepsilon>0$}

Again, the SCM (see §2.6.2) enables the (Online-)efficient estimation of the coercivity constants $\alpha_{a}(\mu)$ and $\alpha_{c}(\mu)$; as we here use the same configurations, we refer to $\S 3.3 .4$ and $\S 5.3 .3$ for details in this context.

To build our low-dimensional reduced basis approximation spaces $X_{N}, Y_{N}, N \in \mathbb{N}_{\max }$, we apply the POD greedy procedure described in Algorithm 6 (see §6.6). The sampling process is based on an exhaustive random sample $\Sigma \subset \mathcal{D}$ of size $|\Sigma|=4,900, \Delta N=2, \delta_{\text {tol }}^{\kappa}=10^{3}$, and the relative reduced basis a posteriori error bound $\Delta_{N}(\mu)=\Delta_{N}^{\varepsilon, K}(\mu) /\left\|\left(u_{N}^{\varepsilon, j}(\mu)\right)_{j \in \mathbb{K}}\right\|_{\ell^{2}(0, K ; Z)}$ (see (6.21), (6.22)). Figure 6.4 now shows the maximum error $\left\|e_{N}^{\varepsilon}(\mu)\right\|_{\ell^{2}(0, K ; Z)}($ see $(6.9))$ in the reduced basis velocity and pressure approximations together with the associated error bound $\Delta_{N}^{\varepsilon, K}(\mu)$ as functions of the dimension $N_{Z}$ for different values of $\varepsilon$. Figure 6.5 then presents the maximum error $\left\|e_{N}^{\varepsilon}(\mu)\right\|_{\ell^{2}(0, k ; Z)}$ and associated error bound $\Delta_{N}^{\varepsilon, k}(\mu)$ as functions of $k \in \mathbb{K}$ for several values of $N$; note that the latter are chosen as the values for which the error bounds $\Delta_{N}^{\varepsilon, K}(\mu)$ guarantee a prescribed accuracy of at least $1 \%$ and 

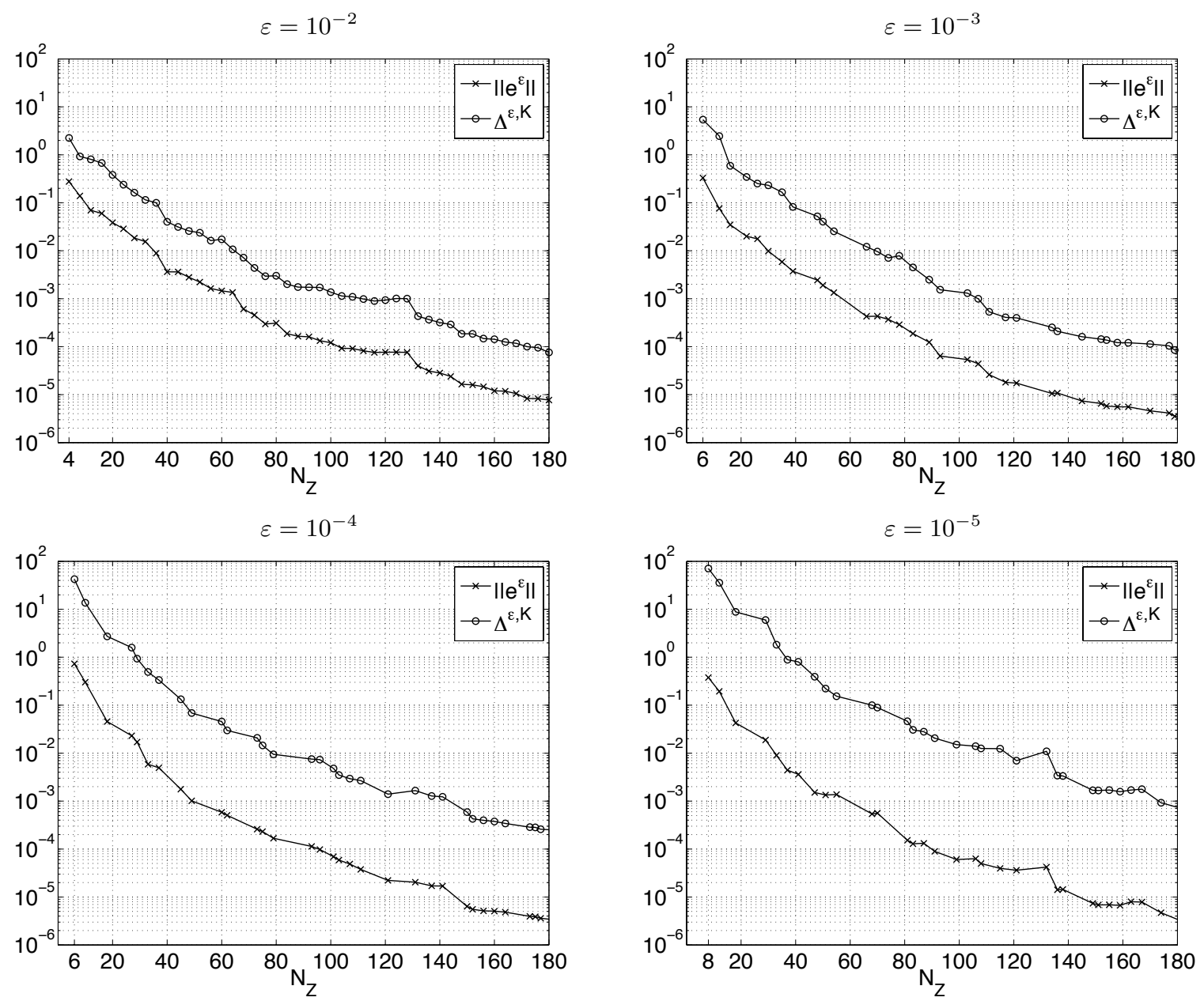

Figure 6.4.: Maximum error $\left\|e_{N}^{\varepsilon}(\mu)\right\|_{\ell^{2}(0, K ; Z)}$ (see (6.9), (6.21)) and maximum error bound $\Delta_{N}^{\varepsilon, K}(\mu)$ (see (6.22)) normalized with respect to $\left\|\left(u^{\varepsilon, j}(\mu), p^{\varepsilon, j}(\mu)\right)_{j \in \mathbb{K}}\right\|_{\ell^{2}(0, K ; Z)}$ shown as functions of $N_{Z}$ for different values of $\varepsilon$; the maximum is taken over 25 parameter values. 
(a) $\varepsilon=10^{-2}$

(b) $\varepsilon=10^{-3}$

(c) $\varepsilon=10^{-4}$

(d) $\varepsilon=10^{-5}$
$N=17\left(N_{Z}=68\right)$

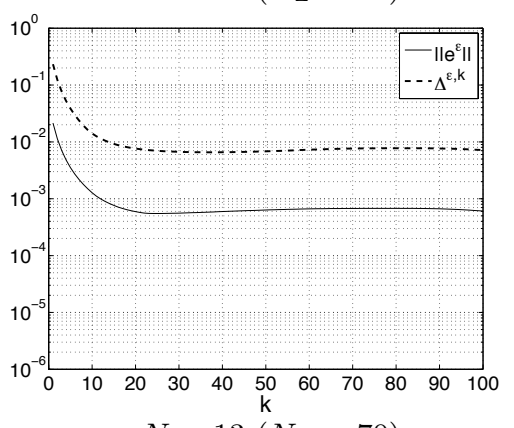

$$
N=13\left(N_{Z}=70\right)
$$

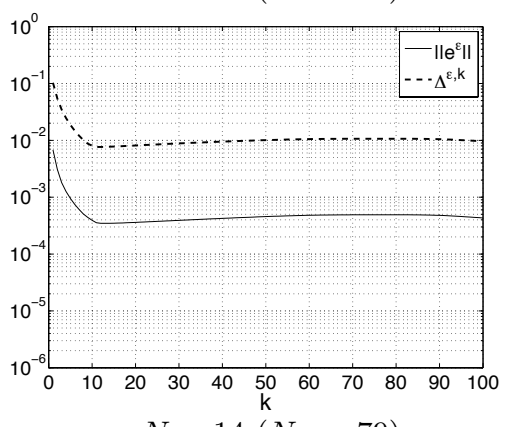

$N=14\left(N_{Z}=79\right)$

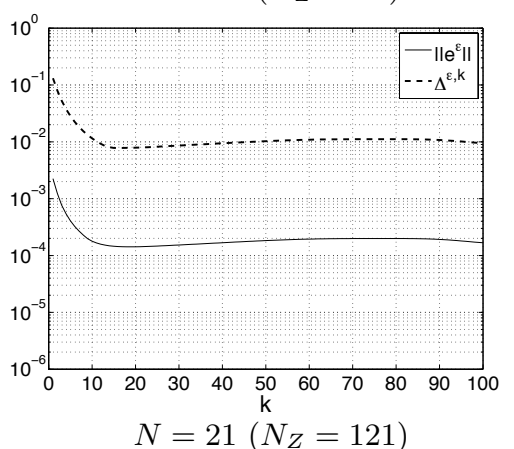

$N=21\left(N_{Z}=121\right)$

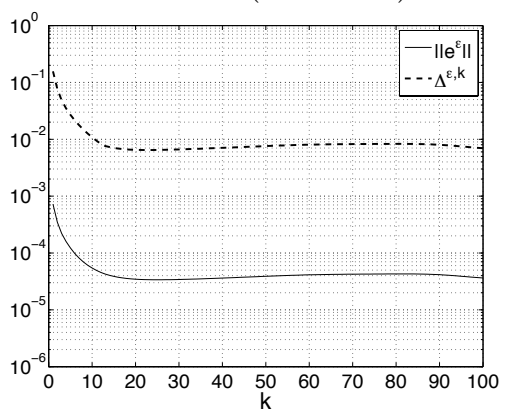

$N=28\left(N_{Z}=112\right)$

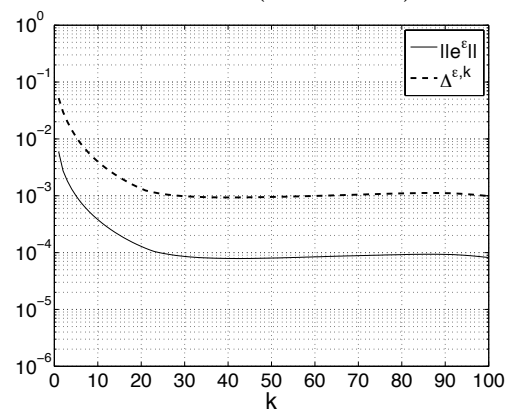

$N=20\left(N_{Z}=107\right)$

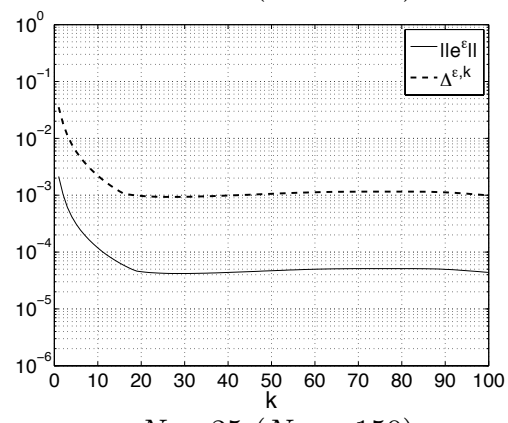

$N=25\left(N_{Z}=150\right)$
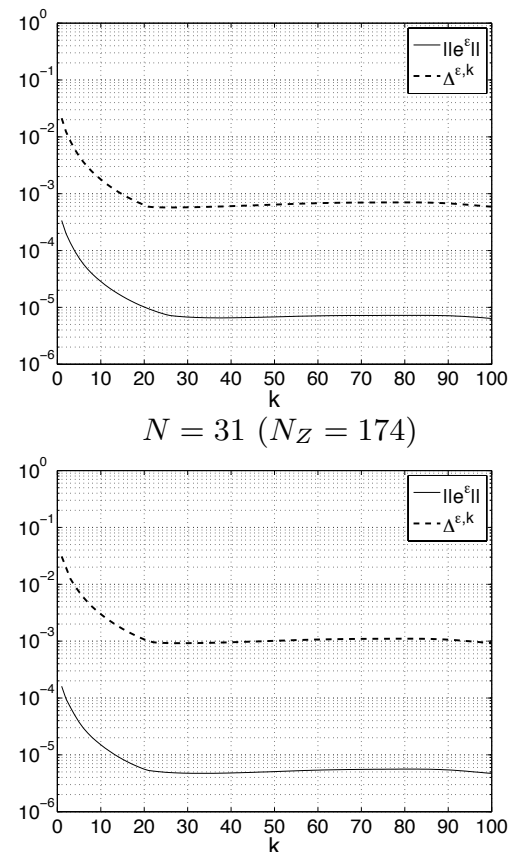

Figure 6.5.: Maximum error $\left\|e_{N}^{\varepsilon}(\mu)\right\|_{\ell^{2}(0, k ; Z)}$ (see (6.9), (6.21)) and maximum error bound $\Delta_{N}^{\varepsilon, k}(\mu)$ (see (6.22)) normalized with respect to $\left\|\left(u^{\varepsilon, j}(\mu), p^{\varepsilon, j}(\mu)\right)_{j \in \mathbb{K}}\right\|_{\ell^{2}(0, k ; Z)}$ shown as functions of $k \in \mathbb{K}$ for several values of $N$ for (a) $\varepsilon=10^{-2}$, (b) $\varepsilon=$ $10^{-3}$, (c) $\varepsilon=10^{-4}$, and (d) $\varepsilon=10^{-5}$; the maximum is taken over 25 parameter values. 
(a) $\varepsilon=10^{-2}$

\begin{tabular}{c|c|c|c|c|c|c|c}
\hline$N$ & $N_{Z}$ & $k=10$ & $k=20$ & $k=40$ & $k=60$ & $k=80$ & $k=100$ \\
\hline 5 & 20 & $1.145 \cdot 10^{1}$ & $1.282 \cdot 10^{1}$ & $1.297 \cdot 10^{1}$ & $1.296 \cdot 10^{1}$ & $1.295 \cdot 10^{1}$ & $1.293 \cdot 10^{1}$ \\
10 & 40 & $1.154 \cdot 10^{1}$ & $1.373 \cdot 10^{1}$ & $1.480 \cdot 10^{1}$ & $1.479 \cdot 10^{1}$ & $1.476 \cdot 10^{1}$ & $1.466 \cdot 10^{1}$ \\
15 & 60 & $1.202 \cdot 10^{1}$ & $1.251 \cdot 10^{1}$ & $1.292 \cdot 10^{1}$ & $1.284 \cdot 10^{1}$ & $1.281 \cdot 10^{1}$ & $1.289 \cdot 10^{1}$ \\
20 & 80 & $1.119 \cdot 10^{1}$ & $1.129 \cdot 10^{1}$ & $1.281 \cdot 10^{1}$ & $1.314 \cdot 10^{1}$ & $1.268 \cdot 10^{1}$ & $1.268 \cdot 10^{1}$ \\
25 & 100 & $1.154 \cdot 10^{1}$ & $1.154 \cdot 10^{1}$ & $1.235 \cdot 10^{1}$ & $1.248 \cdot 10^{1}$ & $1.239 \cdot 10^{1}$ & $1.227 \cdot 10^{1}$ \\
30 & 120 & $1.256 \cdot 10^{1}$ & $1.272 \cdot 10^{1}$ & $1.256 \cdot 10^{1}$ & $1.269 \cdot 10^{1}$ & $1.268 \cdot 10^{1}$ & $1.254 \cdot 10^{1}$ \\
35 & 140 & $1.132 \cdot 10^{1}$ & $1.126 \cdot 10^{1}$ & $1.163 \cdot 10^{1}$ & $1.171 \cdot 10^{1}$ & $1.159 \cdot 10^{1}$ & $1.159 \cdot 10^{1}$ \\
40 & 160 & $1.252 \cdot 10^{1}$ & $1.240 \cdot 10^{1}$ & $1.223 \cdot 10^{1}$ & $1.222 \cdot 10^{1}$ & $1.202 \cdot 10^{1}$ & $1.212 \cdot 10^{1}$ \\
45 & 180 & $1.241 \cdot 10^{1}$ & $1.235 \cdot 10^{1}$ & $1.226 \cdot 10^{1}$ & $1.206 \cdot 10^{1}$ & $1.201 \cdot 10^{1}$ & $1.194 \cdot 10^{1}$ \\
\hline
\end{tabular}

(b) $\varepsilon=10^{-3}$

\begin{tabular}{c|c|c|c|c|c|c|c}
\hline$N$ & $N_{Z}$ & $k=10$ & $k=20$ & $k=40$ & $k=60$ & $k=80$ & $k=100$ \\
\hline 4 & 22 & $2.691 \cdot 10^{1}$ & $3.139 \cdot 10^{1}$ & $3.273 \cdot 10^{1}$ & $3.293 \cdot 10^{1}$ & $3.275 \cdot 10^{1}$ & $3.227 \cdot 10^{1}$ \\
8 & 39 & $2.326 \cdot 10^{1}$ & $2.499 \cdot 10^{1}$ & $2.545 \cdot 10^{1}$ & $2.560 \cdot 10^{1}$ & $2.535 \cdot 10^{1}$ & $2.486 \cdot 10^{1}$ \\
12 & 66 & $2.725 \cdot 10^{1}$ & $2.969 \cdot 10^{1}$ & $3.071 \cdot 10^{1}$ & $3.166 \cdot 10^{1}$ & $3.155 \cdot 10^{1}$ & $3.101 \cdot 10^{1}$ \\
16 & 83 & $2.176 \cdot 10^{1}$ & $2.759 \cdot 10^{1}$ & $2.949 \cdot 10^{1}$ & $3.005 \cdot 10^{1}$ & $3.007 \cdot 10^{1}$ & $2.991 \cdot 10^{1}$ \\
19 & 103 & $2.358 \cdot 10^{1}$ & $2.367 \cdot 10^{1}$ & $2.593 \cdot 10^{1}$ & $2.641 \cdot 10^{1}$ & $2.645 \cdot 10^{1}$ & $2.710 \cdot 10^{1}$ \\
23 & 121 & $2.499 \cdot 10^{1}$ & $2.530 \cdot 10^{1}$ & $2.746 \cdot 10^{1}$ & $2.814 \cdot 10^{1}$ & $2.833 \cdot 10^{1}$ & $2.947 \cdot 10^{1}$ \\
26 & 145 & $2.965 \cdot 10^{1}$ & $2.933 \cdot 10^{1}$ & $2.945 \cdot 10^{1}$ & $2.973 \cdot 10^{1}$ & $2.960 \cdot 10^{1}$ & $2.983 \cdot 10^{1}$ \\
30 & 162 & $2.663 \cdot 10^{1}$ & $2.648 \cdot 10^{1}$ & $2.654 \cdot 10^{1}$ & $2.759 \cdot 10^{1}$ & $2.790 \cdot 10^{1}$ & $2.792 \cdot 10^{1}$ \\
34 & 183 & $2.983 \cdot 10^{1}$ & $2.902 \cdot 10^{1}$ & $2.903 \cdot 10^{1}$ & $2.900 \cdot 10^{1}$ & $2.850 \cdot 10^{1}$ & $2.826 \cdot 10^{1}$ \\
\hline
\end{tabular}

(c) $\varepsilon=10^{-4}$

\begin{tabular}{c|c|c|c|c|c|c|c}
\hline$N$ & $N_{Z}$ & $k=10$ & $k=20$ & $k=40$ & $k=60$ & $k=80$ & $k=100$ \\
\hline 3 & 18 & $8.804 \cdot 10^{1}$ & $8.882 \cdot 10^{1}$ & $8.986 \cdot 10^{1}$ & $9.150 \cdot 10^{1}$ & $9.159 \cdot 10^{1}$ & $9.113 \cdot 10^{1}$ \\
7 & 37 & $7.081 \cdot 10^{1}$ & $7.651 \cdot 10^{1}$ & $7.921 \cdot 10^{1}$ & $8.026 \cdot 10^{1}$ & $8.014 \cdot 10^{1}$ & $7.921 \cdot 10^{1}$ \\
10 & 60 & $6.726 \cdot 10^{1}$ & $8.104 \cdot 10^{1}$ & $9.668 \cdot 10^{1}$ & $9.737 \cdot 10^{1}$ & $9.639 \cdot 10^{1}$ & $9.608 \cdot 10^{1}$ \\
14 & 79 & $7.970 \cdot 10^{1}$ & $9.903 \cdot 10^{1}$ & $1.165 \cdot 10^{2}$ & $1.166 \cdot 10^{2}$ & $1.142 \cdot 10^{2}$ & $1.145 \cdot 10^{2}$ \\
17 & 101 & $9.326 \cdot 10^{1}$ & $1.010 \cdot 10^{2}$ & $1.074 \cdot 10^{2}$ & $1.055 \cdot 10^{2}$ & $1.053 \cdot 10^{2}$ & $1.072 \cdot 10^{2}$ \\
21 & 121 & $1.123 \cdot 10^{2}$ & $1.117 \cdot 10^{2}$ & $1.106 \cdot 10^{2}$ & $1.107 \cdot 10^{2}$ & $1.071 \cdot 10^{2}$ & $1.076 \cdot 10^{2}$ \\
24 & 141 & $1.058 \cdot 10^{2}$ & $1.055 \cdot 10^{2}$ & $1.043 \cdot 10^{2}$ & $1.033 \cdot 10^{2}$ & $9.825 \cdot 10^{1}$ & $9.822 \cdot 10^{1}$ \\
28 & 160 & $9.951 \cdot 10^{1}$ & $9.855 \cdot 10^{1}$ & $9.974 \cdot 10^{1}$ & $9.988 \cdot 10^{1}$ & $9.868 \cdot 10^{1}$ & $9.903 \cdot 10^{1}$ \\
33 & 181 & $8.304 \cdot 10^{1}$ & $8.290 \cdot 10^{1}$ & $8.592 \cdot 10^{1}$ & $8.635 \cdot 10^{1}$ & $8.573 \cdot 10^{1}$ & $8.706 \cdot 10^{1}$ \\
\hline
\end{tabular}

(d) $\varepsilon=10^{-5}$

\begin{tabular}{c|c|c|c|c|c|c|c}
\hline$N$ & $N_{Z}$ & $k=10$ & $k=20$ & $k=40$ & $k=60$ & $k=80$ & $k=100$ \\
\hline 3 & 18 & $2.706 \cdot 10^{2}$ & $2.919 \cdot 10^{2}$ & $3.311 \cdot 10^{2}$ & $3.365 \cdot 10^{2}$ & $3.199 \cdot 10^{2}$ & $3.198 \cdot 10^{2}$ \\
7 & 41 & $2.736 \cdot 10^{2}$ & $2.899 \cdot 10^{2}$ & $2.965 \cdot 10^{2}$ & $2.984 \cdot 10^{2}$ & $2.984 \cdot 10^{2}$ & $2.952 \cdot 10^{2}$ \\
10 & 55 & $2.240 \cdot 10^{2}$ & $2.510 \cdot 10^{2}$ & $2.684 \cdot 10^{2}$ & $2.699 \cdot 10^{2}$ & $2.698 \cdot 10^{2}$ & $2.701 \cdot 10^{2}$ \\
13 & 81 & $3.073 \cdot 10^{2}$ & $3.524 \cdot 10^{2}$ & $3.603 \cdot 10^{2}$ & $3.615 \cdot 10^{2}$ & $3.609 \cdot 10^{2}$ & $3.617 \cdot 10^{2}$ \\
17 & 99 & $2.696 \cdot 10^{2}$ & $2.860 \cdot 10^{2}$ & $3.103 \cdot 10^{2}$ & $3.115 \cdot 10^{2}$ & $3.107 \cdot 10^{2}$ & $3.129 \cdot 10^{2}$ \\
21 & 121 & $3.355 \cdot 10^{2}$ & $3.347 \cdot 10^{2}$ & $3.391 \cdot 10^{2}$ & $3.415 \cdot 10^{2}$ & $3.396 \cdot 10^{2}$ & $3.371 \cdot 10^{2}$ \\
24 & 138 & $2.563 \cdot 10^{2}$ & $2.950 \cdot 10^{2}$ & $3.214 \cdot 10^{2}$ & $3.206 \cdot 10^{2}$ & $3.147 \cdot 10^{2}$ & $3.210 \cdot 10^{2}$ \\
28 & 159 & $2.690 \cdot 10^{2}$ & $2.698 \cdot 10^{2}$ & $2.935 \cdot 10^{2}$ & $2.993 \cdot 10^{2}$ & $2.893 \cdot 10^{2}$ & $3.020 \cdot 10^{2}$ \\
32 & 183 & $2.786 \cdot 10^{2}$ & $2.765 \cdot 10^{2}$ & $3.206 \cdot 10^{2}$ & $3.324 \cdot 10^{2}$ & $3.306 \cdot 10^{2}$ & $3.368 \cdot 10^{2}$ \\
\hline
\end{tabular}

Table 6.2.: Maximum effectivities $\eta_{N}^{\varepsilon, k}(\mu) \equiv \Delta_{N}^{\varepsilon, k}(\mu) /\left\|e_{N}^{\varepsilon}(\mu)\right\|_{\ell^{2}(0, k ; Z)}$ (see (6.23)) for several values of $k \in \mathbb{K}$ and $N$ for (a) $\varepsilon=10^{-2}$, (b) $\varepsilon=10^{-3}$, (c) $\varepsilon=10^{-4}$, and (d) $\varepsilon=10^{-5}$; the maximum is taken over 25 parameter values. 


\begin{tabular}{c|c|c|c|c|c}
\hline$\varepsilon$ & $N_{Z}$ & $N$ & $\left(u_{N}^{\varepsilon, k}(\mu), p_{N}^{\varepsilon, k}(\mu)\right), k \in \mathbb{K}$ & $\Delta_{N}^{\varepsilon, k}(\mu), k \in \mathbb{K}$ & Total \\
\hline $10^{-2}$ & $68(112)$ & $17(28)$ & $3.71(7.65)$ & $14.53(26.95)$ & $18.25(34.60)$ \\
\hline $10^{-3}$ & $70(107)$ & $13(20)$ & $3.99(7.43)$ & $17.19(28.25)$ & $21.18(35.67)$ \\
\hline $10^{-4}$ & $79(150)$ & $14(25)$ & $4.73(13.59)$ & $20.28(44.70)$ & $25.01(58.29)$ \\
\hline $10^{-5}$ & $121(174)$ & $21(31)$ & $9.19(17.47)$ & $33.81(54.08)$ & $43.01(71.55)$ \\
\hline
\end{tabular}

Table 6.3.: Average computation times in milliseconds for the Online evaluation of $\left(u_{N}^{\varepsilon, k}(\mu), p_{N}^{\varepsilon, k}(\mu)\right), k \in \mathbb{K}$, (assembly and solution of (6.7)) and the error bounds $\Delta_{N}^{\varepsilon, k}(\mu), k \in \mathbb{K}$, (see (6.22)) for different values of $\varepsilon$ with a prescribed accuracy of at least $1 \%$ (resp., $0.1 \%$ ) for the reduced basis approximations $\left(u_{N}^{\varepsilon, k}(\mu), p_{N}^{\varepsilon, k}(\mu)\right), k \in \mathbb{K}$.

$0.1 \%$ in the reduced basis approximations. First, we again observe that the reduced basis error and error bounds are roughly uniform in time (see Fig. 6.5) and decrease rapidly as $N_{Z}$ increases (see Fig. 6.4). We obtain stable reduced basis approximations whose rapid convergence is not affected by the penalty parameter, and a posteriori error bounds that are meaningful and rigorous. Second, using the condition numbers $\kappa_{N}^{\varepsilon}(\mu)$ as an indicator for an ill-conditioned system, Algorithm 6 guarantees stability by properly accounting for the effects of the penalty term: For $\varepsilon=10^{-2}$, the sampling process recognizes that the reduced basis approximation spaces $X_{N}, Y_{N}$ do not have to be stabilized to provide accurate approximations; taking smaller values of $\varepsilon$ and thus approaching the nonpenalized problem, an additional enrichment of the reduced basis approximation space for the velocity then becomes more and more necessary. Third, we see that the error bounds are tight for $\varepsilon=10^{-2}$ but become less sharp as we decrease $\varepsilon$ and our perturbed truth approximation becomes more accurate. However, effectivities exhibit a similar $O\left(\frac{1}{\sqrt{\varepsilon}}\right)$-dependence on the penalty parameter as observed in the stationary case (see §5.3.3) and remain reasonably small for relatively small values of $\varepsilon$. To further quantify this statement, we present in Table 6.2 the effectivities associated with $\Delta_{N}^{\varepsilon, k}(\mu)$ for different values of $k, N$, and $\varepsilon$. We note that their values are fairly constant with $k$ and $N$ and confirm the $O\left(\frac{1}{\sqrt{\varepsilon}}\right)$-dependence indicated by Fig. 6.4 and Fig. 6.5. As before, the effects of the penalty parameter on the effectivities are thus relatively benign and we obtain useful bounds for reasonably small values of $\varepsilon$.

We close this section by discussing the Online computation times. For comparison, once the $\mu$-independent parts in (2.7) have been formed, direct computation of the truth approximation $\left(u^{\varepsilon, k}(\mu), p^{\varepsilon, k}(\mu)\right), k \in \mathbb{K}$, (i.e., assembly and solution of (6.6)) requires roughly 23 seconds on a $2.66 \mathrm{GHz}$ Intel Core 2 Duo processor. Again, our rigorous and inexpensive reduced basis a posteriori error bounds enable us to choose the reduced basis dimension just large enough to obtain a desired accuracy. Choosing $\varepsilon=10^{-2}$, the error bounds $\Delta_{N}^{\varepsilon, k}(\mu)$ are sharp with effectivities of approximately 12 (see Table 6.2(a)) and prescribe a dimension of $N_{Z}=68$ to achieve an accuracy of at least $1 \%$ in the reduced basis approximations $\left(u_{N}^{\varepsilon, k}(\mu), p_{N}^{\varepsilon, k}(\mu)\right), k \in \mathbb{K}$ (see Fig. 6.4). Once the database has been loaded, the Online calculation of $\left(u_{N}^{\varepsilon, k}(\mu), p_{N}^{\varepsilon, k}(\mu)\right), k \in \mathbb{K}$, (i.e., assembly and solution of $\left.(6.7)\right)$ and $\Delta_{N}^{\varepsilon, k}(\mu), k \in \mathbb{K}$, for any new value of $\mu \in \mathcal{D}$ then takes on average 3.71 and 14.53 milliseconds, respectively, which is in total roughly 1,200 times faster than direct computation of the truth approximation. Choosing smaller values for $\varepsilon$, the error bounds become more pessimistic and thus 
dictate a larger system dimension at which they guarantee the same order of accuracy. For $\varepsilon=10^{-5}$, we need $N_{Z}=121$ to achieve a prescribed accuracy of at least $1 \%$ in the reduced basis approximations (see Fig. 6.4); the Online calculation of $\left(u_{N}^{\varepsilon, k}(\mu), p_{N}^{\varepsilon, k}(\mu)\right), k \in \mathbb{K}$, and $\Delta_{N}^{\varepsilon, k}(\mu), k \in \mathbb{K}$, then takes on average 9.19 and 33.81 milliseconds, respectively, which is in total roughly 500 times faster than direct computation of the truth approximation. Thus, even for small penalty parameters $\varepsilon$, accurate approximations are guaranteed at significant Online savings. Detailed computation times for different values of $\varepsilon$ are given in Table 6.3.

\subsection{Concluding Remarks}

In this chapter, we present new reduced basis methods for the instationary Stokes equations.

Combining techniques developed in Chapter 2 with current reduced basis approaches for parabolic problems, we derive new rigorous a posteriori bounds for the errors in the reduced basis velocity approximations and a POD greedy procedure that properly accounts for temporal and parametric causality as well as stability. The method provides rapidly convergent reduced basis approximations that are highly efficient and whose accuracy is certified by sharp and inexpensive a posteriori error bounds.

An approximation by penalty or regularization again allows for significant Offline savings at the expense of a less accurate truth approximation. Due to the introduced penalty term, an additional enrichment of the reduced basis velocity approximation space is not always necessary to obtain stable approximations; moreover, we obtain a posteriori error bounds that do not involve the expensive computation of inf-sup stability constants. As in the stationary case (see Chapter 5), the method provides reduced basis approximations and meaningful a posteriori error bounds that are computed very easily; nevertheless, drawbacks such as the disadvantageous dependence of the error bounds on the penalty parameter remain.

Time integration is achieved through a backward Euler method. Clearly, also other time integration schemes may be used. Using a Crank-Nicolson method, often preferred in practice due to its second-order accuracy, we may develop a penalty approach that is very similar to the one presented in this chapter and which is therefore included in Appendix C; in case of $\varepsilon=0$, useful reduced basis a posteriori error bounds could not yet been derived and may therefore be - as well as a posteriori error bounds for the reduced basis pressure approximations - part of future work. 



\section{References}

[1] R. A. Adams And J. J. F. Fournier, Sobolev Spaces, Pure Appl. Math. 140, Elsevier, Oxford, 2nd ed., 2003.

[2] B. O. Almroth, P. Stern, And F. A. Brogan, Automatic choice of global shape functions in structural analysis, AIAA J., 16 (1978), pp. 525-528.

[3] A. C. Antoulas And D. C. Sorensen, Approximation of large-scale dynamical systems: An overview, tech. report, Rice University, Houston, TX, 2001.

[4] A. C. Antoulas, D. C. Sorensen, and S. Gugercin, A survey of model reduction methods for large-scale systems, Contemp. Math., 280 (2001), pp. 193-219.

[5] I. BABUŠKA, Error bound for finite element method, Numer. Math., 16 (1971), pp. 322 333.

[6] Z. BAI, Krylov subspace techniques for reduced-order modeling of large-scale dynamical systems, Appl. Numer. Math., 43 (2002), pp. 9-44.

[7] M. Barrault, N. C. Nguyen, Y. Maday, and A. T. Patera, An "empirical interpolation" method: Application to efficient reduced-basis discretization of partial differential equations, C. R. Acad. Sci. Paris, Ser. I 339 (2004), pp. 667-672.

[8] P. Benner, Numerical linear algebra for model reduction in control and simulation, tech. report, Technische Universität Chemnitz, Chemnitz, 2005.

[9] M. Bercovier, Perturbation of a mixed variational problem. Application to mixed finite element methods, R.A.I.R.O. Anal. Numer., 12 (1978), pp. 211-236.

[10] P. Binev, A. Cohen, W. Dahmen, R. DeVore, G. Petrova, and P. WojTASZCZYK, Convergence rates for greedy algorithms in reduced basis methods, submitted.

[11] S. Boyaval, C. Le Bris, T. Lelièvre, Y. Maday, N.-C. Nguyen, and A. T. PAterA, Reduced basis techniques for stochastic problems, Arch. Comput. Methods Eng., 17 (2012), pp. 435-454.

[12] F. BREzZI, On the existence, uniqueness and approximation of saddle-point problems arising from Lagrangian multipliers, R.A.I.R.O. Anal. Numer., 8 (1974), pp. 129-151. 
[13] F. Brezzi and M. Fortin, Mixed and Hybrid Finite Element Methods, Springer Ser. Comput. Math. 15, Springer-Verlag, New York, 1991.

[14] H. Bruus, Theoretical Microfluidics, Oxf. Master Ser. Condens. Matter Phys., Oxford Univ. Press, Oxford, 2008.

[15] A. Buffa, Y. Maday, A. T. Patera, C. Prud'homme, and G. Turinici, A priori convergence of the greedy algorithm for the parametrized reduced basis, M2AN Math. Model. Numer. Anal., 46 (2012), pp. 595-603.

[16] T. Bui-Thanh, M. Damodaran, and K. Willcox, Proper orthogonal decomposition extensions for parametric applications in transonic aerodynamics (AIAA Paper 2003-4213), in Proceedings of the 15th AIAA Computational Fluid Dynamics Conference, 2003.

[17] T. Bui-Thanh, K. Willcox, And O. Ghattas, Model reduction for large-scale systems with high-dimensional parametric input space, SIAM J. Sci. Comput., 30 (2008), pp. 3270-3288.

[18] C. J. Campbell and B. A. Grzybowski, Microfluidic mixers: From microfabricated to self-assembling devices, Philos. Trans. R. Soc. Lond. Ser. A Math. Phys. Eng. Sci., (2004), pp. 1069-1086.

[19] C. Canuto, T. Tonn, and K. Unban, A-posteriori error analysis of the reduced basis method for non-affine parametrized nonlinear PDE's, SIAM J. Numer. Anal., 47 (2009), pp. 2001-2022.

[20] G. F. Carey and R. Krishnan, Penalty approximation of Stokes flow, Comp. Meth. Appl. Mech. Eng., 35 (1982), pp. 169-206.

[21] A. ChatterJee, An introduction to the proper orthogonal decomposition, Current Sci., 78 (2000), pp. 808-817.

[22] Y. Chen, J. S. Hesthaven, Y. Maday, and J. Rodriguez, Certified reduced basis methods and output bounds for the harmonic Maxwell's equations, SIAM J. Sci. Comput., 32 (2012), pp. 970-996.

[23] W. Dahmen, C. Plesken, and G. Welper, Double greedy algorithms: Reduced basis methods for transport dominated problems, in preparation.

[24] L. DEDÈ, Reduced basis method and a posteriori error estimation for parametrized linear-quadratic optimal control problems, SIAM J. Sci. Comput., 32 (2010), pp. 9971019.

[25] S. Deparis And G. RozzA, Reduced basis method for multi-parameter dependent steady Navier-Stokes equations: Applications to natural convection in a cavity, J. Comput. Phys., 228 (2009), pp. 4359-4378.

[26] J. L. Eftang, M. A. Grepl, and A.T. Patera, A posteriori error bounds for the empirical interpolation method, C. R. Acad. Sci. Paris, Ser. I 348 (2012), pp. 575-579. 
[27] A. Ern And J.-L. Guermond, Theory and Practice of Finite Elements, Appl. Math. Sci. 159, Springer-Verlag, New York, 2004.

[28] J. P. Fink And W. C. Rheinboldt, On the error behavior of the reduced basis technique for nonlinear finite element approximations, ZAMM Z. Angew. Math. Mech., 63 (1983), pp. 21-28.

[29] — Local error estimates for parametrized nonlinear equations, SIAM J. Numer. Anal., 22 (1985), pp. 729-735.

[30] R. W. Freund, Model reduction methods based on Krylov subspaces, Acta Numer., 12 (2003), pp. 267-319.

[31] A.-L. Gerner And K. Veroy, Certified reduced basis methods for parametrized saddle point problems, SIAM J. Sci. Comput., accepted.

[32] — Certified reduced basis methods for the instationary Stokes equations, submitted.

[33] __ Reduced basis a posteriori error bounds for symmetric parametrized saddle point problems, submitted.

[34] _ Reduced basis a posteriori error bounds for the instationary Stokes equations: A penalty approach, in MATHMOD 2012 Conference Proceedings, accepted.

[35] —_ Reduced basis a posteriori error bounds for the Stokes equations in parametrized domains: A penalty approach, Math. Models Methods Appl. Sci., 21 (2011), pp. 21032134 .

[36] V. Girault And P.-A. Raviart, Finite Element Methods for Navier-Stokes Equations: Theory and Algorithms, Springer Ser. Comput. Math. 5, Springer-Verlag, Berlin, 1986.

[37] M. A. Grepl, Reduced-Basis Approximations and A Posteriori Error Estimation for Parabolic Partial Differential Equations, PhD thesis, Massachusetts Institute of Technology, Cambridge, MA, 2005.

[38] _ Certified reduced basis methods for nonaffine linear time-varying and nonlinear parabolic partial differential equations, Math. Models Methods Appl. Sci., 22 (2012).

[39] M. A. GRepl AND M. KÄRCHeR, Reduced basis a posteriori error bounds for parametrized linear-quadratic elliptic optimal control problems, C. R. Acad. Sci. Paris, Ser. I 349 (2011), pp. 873-877.

[40] M. A. Grepl, Y. Maday, N.-C. NGuyen, and A. T. Patera, Efficient reducedbasis treatment of nonaffine and nonlinear partial differential equations, M2AN Math. Model. Numer. Anal., 41 (2007), pp. 575-605.

[41] M. A. Grepl and A. T. PATera, A posteriori error bounds for reduced-basis approximations of parametrized parabolic partial differential equations, M2AN Math. Model. Numer. Anal., 39 (2005), pp. 157-181. 
[42] S. Gross And A. Reusken, Numerical Methods for Two-phase Incompressible Flows, Springer Ser. Comput. Math. 40, Springer-Verlag, Berlin, 2011.

[43] S. Gugercin And A. C. Antoulas, A survey of model reduction by balanced truncation and some new results, Internat. J. Control, 8 (2004), pp. 748-766.

[44] M. Gunzburger, J. Peterson, And J. Shadid, Reduced-order modeling of timedependent PDEs with multiple parameters in the boundary data, Comput. Methods Appl. Mech. Engrg., 196 (2007), pp. 1030-1047.

[45] M. D. Gunzburger, Finite Element Methods for Viscous Incompressible Flows: A Guide to Theory, Practice, and Algorithms, Academic Press, Boston, 1989.

[46] B. HaAsdonk, Convergence rates for the POD-greedy method, M2AN Math. Model. Numer. Anal., accepted.

[47] B. HaAsdonk And M. Ohlberger, Reduced basis method for finite volume approximations of parametrized linear evolution equations, M2AN Math. Model. Numer. Anal., 42 (2008), pp. 277-302.

[48] B. HaAsdonk, J. Salomon, and B. Wohlmuth, A reduced basis method for parametrized variational inequalities, SIAM J. Numer. Anal., accepted.

[49] B. HaAsdonk, K. URban, And B. Wieland, Reduced basis methods for parametrized partial differential equations with stochastic influences using the Karhunen-Loève expansion, submitted.

[50] J. Happel and H. Brenner, Low Reynolds number hydrodynamics: With special applications to particulate media, Martinus Nijhoff Publishers, The Hague, 1983.

[51] J. C. Heinrich And C. A. Vionnet, The penalty method for the Navier-Stokes equations, Arch. Comput. Methods Eng., 2 (1995), pp. 51-65.

[52] T. J. R. Hughes, W. K. Liu, And A. Brooks, Finite element analysis of incompressible viscous flows by the penalty function formulation, J. Comput. Phys., 30 (1979), pp. 1-60.

[53] D. B. P. Huynh, D. J. Knezevic, Y. Chen, J. S. Hesthaven, and A. T. PatERA, A natural-norm successive constraint method for inf-sup lower bounds, Comput. Methods Appl. Mech. Engrg., 199 (2010), pp. 1963-1975.

[54] D. B. P. Huynh, D. J. Knezevic, And A. T. PAtera, A static condensation reduced basis element method: Approximation and a posteriori error estimation, M2AN Math. Model. Numer. Anal., accepted.

[55] D. B. P. Huynh, G. Rozza, S. Sen, and A. T. Patera, A successive constraint linear optimization method for lower bounds of parametric coercivity and inf-sup stability constants, C. R. Acad. Sci. Paris, Ser. I 345 (2007), pp. 473-478. 
[56] K. Ito And S. S. Ravindran, A reduced basis method for control problems governed by PDEs, in Control and Estimation of Distributed Parameter Systems, Internat. Ser. Numer. Math. 126, Birkhäuser, 1998, pp. 153-168.

[57] — A reduced-order method for simulation and control of fluid flows, J. Comput. Phys., 143 (1998), pp. 403-425.

[58] _ _ Reduced basis method for optimal control of unsteady viscous flows, Int. J. Comput. Fluid Dyn., 15 (2001), pp. 97-113.

[59] N. Jung, Error Estimation for Parametric Model Order Reduction and its Application, PhD thesis, Technische Universität München, Munich, 2012.

[60] N. Jung, A. T. Patera, B. HaAsdonk, And B. Lohmann, Model order reduction and error estimation with an application to the parameter-dependent Eddy Current equation, Math. Comput. Model. Dyn. Syst., 17 (2011), pp. 561-582.

[61] M. Kärcher And M. A. Grepl, A certified reduced basis method for parametrized elliptic optimal control problems, submitted.

[62] B. S. Kirk, J. W. Peterson, R. H. Stogner, and G. F. Carey, libMesh: A $C++$ library for parallel adaptive mesh refinement/coarsening simulations, Engineering with Computers, 22 (2006), pp. 237-254.

[63] D. J. Knezevic, N.-C. NGuyen, And A. T. Patera, Reduced basis approximation and a posteriori error estimation for the parametrized unsteady Boussinesq equations, Math. Models Methods Appl. Sci., 21 (2011), pp. 1415-1442.

[64] D. J. Knezevic And A. T. Patera, A certified reduced basis method for the FokkerPlanck equation of dilute polymeric fluids: FENE dumbbells in extensional flow, SIAM J. Sci. Comput., 32 (2010), pp. 793-817.

[65] D. J. Knezevic and J. W. Peterson, A high-performance parallel implementation of the certified reduced basis method, Comput. Methods Appl. Mech. Engrg., 200 (2011), pp. 1455-1466.

[66] K. Kunisch And S. Volkwein, Augmented Lagrangian-SQP techniques and their approximations, in Optimization Methods in Partial Differential Equations, Contemp. Math. 209, Amer. Math. Soc., 1997, pp. 147-159.

[67] _ Control of the Burger's equation by a reduced-order approach using proper orthogonal decomposition, J. Optim. Theory Appl., 102 (1999), pp. 345-371.

[68] —_ Galerkin proper orthogonal decomposition methods for parabolic problems, Numer. Math., 90 (2001), pp. 117-148.

[69] - Galerkin proper orthogonal decomposition methods for a general equation in fluid dynamics, SIAM J. Numer. Anal., 40 (2002), pp. 492-515. 
[70] A. E. Løvgren, Y. Maday, and E. M. Rønquist, The reduced basis element method for fluid flows, in Analysis and Simulation of Fluid Dynamics, Adv. Math. Fluid Mech., Birkhäuser, 2006, pp. 129-154.

[71] L. Machiels, Y. Maday, I. B. Oliveira, A. T. Patera, and D. Rovas, Output bounds for reduced-basis approximations of symmetric positive definite eigenvalue problems, C. R. Acad. Sci. Paris, Ser. I 331 (2000), pp. 153-158.

[72] Y. Maday, A. T. Patera, And D. V. Rovas, A blackbox reduced-basis output bound method for noncoercive linear problems, in Nonlinear Partial Differential Equations and their Applications - Collège de France Seminar Volume XIV, Studies in Mathematics and its Applications 31, Elsevier, 2002, pp. 533-569.

[73] Y. Maday, A. T. Patera, and G. Turinici, A priori convergence theory for approximation of single-parameter elliptic partial differential equations, C.R. Acad. Sci. Paris, Ser. I 335 (2002), pp. 289-294.

[74] Y. Maday and E. Rønquist, A reduced-basis element method, J. Sci. Comput., 17 (2002), pp. 447-459.

[75] A. Manzoni, A. Quarteroni, And G. Rozza, Shape optimization for viscous flows by reduced basis methods and free-form deformation, Internat. J. Numer. Methods Fluids, in press.

[76] - Model reduction techniques for fast blood flow simulation in parametrized geometries, Int. J. Numer. Methods Biomed. Eng., 28 (2012), pp. 604-625.

[77] F. Negri, Reduced Basis Method for Parametrized Optimal Control Problems Governed by PDEs, master's thesis, Politecnico di Milano, Milano, 2011.

[78] N.-C. NGuyen, G. Rozza, And A. T. Patera, Reduced basis approximation and a posteriori error estimation for the time-dependent viscous Burgers' equation, Calcolo, 46 (2009), pp. 157-185.

[79] N.-C. Nguyen, K. Veroy, And A. T. Patera, Certified real-time solution of parametrized partial differential equations, in Handbook of Materials Modeling, Springer-Verlag, 2005, pp. 1523-1558.

[80] A. K. Noor And J. M. Peters, Reduced basis technique for nonlinear analysis of structures, AIAA Journal, 18 (1980), pp. 455-462.

[81] J. T. Oden And O. Jacquotte, Stable and unstable RIP/perturbed Lagrangian methods for two-dimensional viscous flow problems, in Finite Elements in Fluids V, John Wiley and Sons, 1984.

[82] J. T. Oden, N. Kikuchi, And Y. J. Song, Penalty finite element methods for Stokesian flows, Comput. Methods Appl. Mech. Engrg., 31 (1982), pp. 297-329.

[83] A. T. Patera And G. Rozza, Reduced Basis Methods and A Posteriori Error Estimation for Parametrized Partial Differential Equations, MIT Pappalardo Graduate Monographs in Mechanical Engineering, Cambridge, MA, 2006-2008. 
[84] J. S. PETERson, The reduced basis method for incompressible viscous flow calculations, SIAM J. Sci. Stat. Comput., 10 (1989), pp. 777-786.

[85] T. A. Porsching, Estimation of the error in the reduced basis method solution of nonlinear equations, Math. Comp., 45 (1985), pp. 487-496.

[86] T. A. Porsching And M. Lin Lee, The reduced basis method for initial value problems, SIAM J. Numer. Anal., 24 (1987), pp. 1277-1287.

[87] C. Prud'homme, D. Rovas, K. Veroy, Y. Maday, A. T. Patera, and G. TURINICI, Reliable real-time solution of parametrized partial differential equations: Reduced-basis output bound methods, J. Fluids Engineering, 124 (2002), pp. 70-80.

[88] A. Quarteroni And G. Rozza, Numerical solution of parametrized Navier-Stokes equations by reduced basis methods, Numer. Methods Partial Differential Equations, 23 (2007), pp. 923-948.

[89] A. Quarteroni And A. Valli, Numerical Approximation of Partial Differential Equations, Springer Ser. Comput. Math. 23, Springer-Verlag, Berlin, 2008.

[90] B. RAHn, A balanced truncation primer, tech. report, California Institute of Technology, Pasadena, CA, 2001.

[91] J. N. REDDY, On penalty function methods in the finite-element analysis of flow problems, Internat. J. Numer. Methods Fluids, 2 (1982), pp. 151-171.

[92] D. V. Rovas, Reduced-Basis Output Bound Methods for Parametrized Partial Differential Equations, PhD thesis, Massachusetts Institute of Technology, Cambridge, MA, 2003.

[93] C. W. Rowley, Model reduction for fluids, using balanced proper orthogonal decomposition, Internat. J. Bifur. Chaos Appl. Sci. Engrg., 15 (2005), pp. 997-1013.

[94] G. RozzA, Reduced basis methods for Stokes equations in domains with non-affine parameter dependence, Comput. Vis. Sci., 12 (2009), pp. 23-35.

[95] G. Rozza, D. B. P. Huynh, And A. T. PAterA, Reduced basis approximation and a posteriori error estimation for affinely parametrized elliptic coercive partial differential equations, Arch. Comput. Methods Eng., 15 (2008), pp. 229-275.

[96] G. Rozza And K. Veroy, On the stability of the reduced basis method for Stokes equations in parametrized domains, Comput. Methods Appl. Mech. Engrg., 196 (2007), pp. $1244-1260$.

[97] S. Sen, K. Veroy, D. B. P. Huynh, S. Deparis, N.-C. Nguyen, and A. T. PaTERA, "Natural norm" a posteriori error estimators for reduced basis approximations, J. Comput. Phys., 217 (2006), pp. 37-62.

[98] T. M. Squires And S. R. Quake, Microfluidics: Fluid physics at the nanoliter scale, Rev. Modern Phys., 77 (2005), pp. 977-1026. 
[99] H. A. Stone, A. D. Stroock, And A. Ajdari, Engineering flows in small devices: Microfluidics toward a lab-on-a-chip, Annu. Rev. Fluid Mech., 36 (2004), pp. 381-411.

[100] C. TAYlor And P. Hood, A numerical solution of the Navier-Stokes equations using the finite element technique, Comput. \& Fluids, 1 (1973), pp. 73-100.

[101] R. Temam, Navier-Stokes Equations: Theory and Numerical Analysis, Studies in Mathematics and its Applications 2, North-Holland, Amsterdam, rev. ed., 1979.

[102] V. Thomée, Galerkin Finite Element Methods for Parabolic Problems, Springer Ser. Comput. Math. 25, Springer-Verlag, Berlin, 1997.

[103] T. Tonn, K. Urban, And S. Volkwein, Comparison of the reduced-basis and POD a-posteriori error estimators for an elliptic linear-quadratic optimal control problem, Math. Comput. Model. Dyn. Syst., 17 (2011), pp. 355-369.

[104] K. Veroy And A. T. Patera, Certified real-time solution of the parametrized steady incompressible Navier-Stokes equations: Rigorous reduced-basis a posteriori error bounds, Internat. J. Numer. Methods Fluids, 47 (2005), pp. 773-788.

[105] K. Veroy, C. Prud'homme, and A. T. Patera, Reduced-basis approximation of the viscous Burgers equation: Rigorous a posteriori error bounds, C. R. Acad. Sci. Paris, Ser. I 337 (2003), pp. 619-624.

[106] K. Veroy, C. Prud'homme, D. V. Rovas, and A. T. Patera, A posteriori error bounds for reduced-basis approximation of parametrized noncoercive and nonlinear elliptic partial differential equations (AIAA Paper 2003-3847), in Proceedings of the 16th AIAA Computational Fluid Dynamics Conference, 2003.

[107] G. Vossen And S. Volkwein, Model reduction techniques with a-posteriori error analysis for linear-quadratic optimal control problems, Numerical Algebra, Control and Optimization (NACO), 2 (2012), pp. 465-485.

[108] K. Willcox And J. Peraire, Balanced model reduction via the proper orthogonal decomposition, AIAA Journal, 40 (2002), pp. 2323-2330.

[109] J. Xu And L. Zikatanov, Some observations on Babuška and Brezzi theories, Numer. Math., 94 (2003), pp. 195-202.

[110] M. Yano, K. Urban, And A. T. Patera, A space-time certified reduced basis method for Burgers' equation, submitted.

[111] L. Zanon, Reduced-Basis Approximation and A Posteriori Error Estimation for Saddle-Point Problems, master's thesis, Politecnico di Torino, Torino, 2010.

[112] O. C. Zienkewicz, R. L. Taylor, and J. M. Too, Reduced integration technique in general analysis of plates and shells, Internat. J. Numer. Methods Engrg., 3 (1971), pp. 275-290. 


\section{Appendix A}

\section{Notations}

In this section, we collect some basic notation that is used throughout this work.

\section{Vectors and Matrices}

The standard sets of numbers are denoted by $\mathbb{N}, \mathbb{N}_{0} \equiv \mathbb{N} \cup\{0\}$, and $\mathbb{R}$. Let $n, m \in \mathbb{N}$. For a vector $x \in \mathbb{R}^{n},|x|=\sqrt{x \cdot x}$ denotes the Euclidean norm. For a matrix $A=\left(A_{i j}\right) \in \mathbb{R}^{m \times n}$, $\operatorname{det}(A)$ refers to the determinant and $\sigma^{\max }(A), \sigma^{\min }(A)$ to the maximum and minimum singular values, respectively. The matrix norms $\|\cdot\|_{2},\|\cdot\|_{1}$, and $\|\cdot\|_{\infty}$ are defined as

$$
\|A\|_{2} \equiv \sup _{x \in \mathbb{R}^{n}} \frac{|A x|}{|x|}=\sigma^{\max }(A), \quad\|A\|_{1} \equiv \max _{j=1, \ldots, n} \sum_{i=1}^{m}\left|A_{i j}\right|, \quad\|A\|_{\infty} \equiv \max _{i=1, \ldots, m} \sum_{j=1}^{n} A_{i j} ;
$$

here and throughout this thesis, we suppress the obvious requirement of nonzero elements in the denominators for clarity of exposition. Note that in particular $\|A\|_{2} \leq \sqrt{\|A\|_{1}\|A\|_{\infty}}$ holds true. For a nonsingular matrix, we also consider the condition number $\kappa(A)$,

$$
\kappa(A) \equiv\|A\|_{2}\left\|A^{-1}\right\|_{2}=\frac{\sigma^{\max }(A)}{\sigma^{\min }(A)} .
$$

\section{Functions}

Let $\Omega \subseteq \mathbb{R}^{d}, d \in \mathbb{N}$, be a bounded domain. Then, its boundary is denoted by $\Gamma \equiv \partial \Omega$ and $\bar{\Omega} \equiv \Omega \cup \Gamma$ refers to the closure of $\Omega$. For a scalar function $p: \Omega \rightarrow \mathbb{R}, \frac{\partial p}{\partial x_{i}}$ denotes the partial derivative with respect to the variable $x_{i}, i=1, \ldots, d$. The gradient, normal derivative, and Laplacian are then given by

$$
\nabla p \equiv\left(\frac{\partial p}{\partial x_{i}}\right)_{i=1}^{d}, \quad \frac{\partial p}{\partial n} \equiv \nabla p \cdot n, \quad \Delta p \equiv \frac{\partial^{2} p}{\partial x_{i} \partial x_{i}}
$$

respectively, where $n$ denotes the unit outward normal to $\Gamma$; here and throughout this thesis, repeated indices imply summation. For a vector function $u=\left(u_{1}, \ldots, u_{d}\right): \Omega \rightarrow \mathbb{R}^{d}$, partial derivatives are defined componentwise, i.e.,

$$
\frac{\partial u}{\partial x_{i}} \equiv\left(\frac{\partial u_{j}}{\partial x_{i}}\right)_{j=1}^{d}, i=1, \ldots, d, \quad \frac{\partial u}{\partial n} \equiv\left(\frac{\partial u_{j}}{\partial n}\right)_{j=1}^{d}, \quad \Delta u \equiv\left(\Delta u_{j}\right)_{j=1}^{d},
$$


and we further consider the divergence operator

$$
\operatorname{div}(u) \equiv \nabla \cdot u \equiv \frac{\partial u_{i}}{\partial x_{i}}
$$

In this work, $\tilde{\Omega} \equiv \Omega(\mu)$ typically refers to a domain whose exact geometric configuration is specified by a parameter $\mu \in \mathbb{R}^{n}, n \in \mathbb{N}$; its boundary is then denoted by $\tilde{\Gamma} \equiv \partial \tilde{\Omega}$ with unit outward normal $\tilde{n}$. According to the above notations, $\tilde{\nabla}, \frac{\partial}{\partial \tilde{n}}$, and $\tilde{\Delta}$ refer to the gradient, normal derivative, and Laplace operator over $\tilde{\Omega}$,

$$
\begin{aligned}
& \tilde{\nabla} \tilde{p} \equiv\left(\frac{\partial \tilde{p}}{\partial \tilde{x}_{i}}\right)_{i=1}^{d}, \quad \frac{\partial \tilde{p}}{\partial \tilde{n}}=\tilde{\nabla} \tilde{p} \cdot \tilde{n}, \quad \tilde{\Delta} \tilde{p} \equiv \frac{\partial^{2} \tilde{p}}{\partial \tilde{x}_{i} \partial \tilde{x}_{i}}, \\
& \frac{\partial \tilde{u}}{\partial \tilde{n}} \equiv\left(\frac{\partial \tilde{u}_{j}}{\partial \tilde{n}}\right)_{j=1}^{d}, \quad \tilde{\Delta} \tilde{u}=\left(\tilde{\Delta} \tilde{u}_{j}\right)_{j=1}^{d}, \quad \tilde{\nabla} \cdot \tilde{u} \equiv \frac{\partial \tilde{u}_{i}}{\partial \tilde{x}_{i}},
\end{aligned}
$$

for scalar and vector functions $\tilde{p}: \tilde{\Omega} \rightarrow \mathbb{R}$ and $\tilde{u}=\left(\tilde{u}_{1}, \ldots, \tilde{u}_{d}\right): \tilde{\Omega} \rightarrow \mathbb{R}^{d}$.

\section{Function Spaces}

The space of continuous functions on $\Omega$ is given by $C(\Omega)$; for $j \in \mathbb{N}, C^{j}(\Omega)$ consists of all functions that are $j$-times continuously differentiable on $\Omega$. We denote by $L^{2}(\Omega)$ the standard Lebesgue spaces of square integrable functions over $\Omega$, and $H^{1}(\Omega) \equiv\left\{v \in L^{2}(\Omega) \mid\right.$ $\left.\nabla v \in\left(L^{2}(\Omega)\right)^{2}\right\}$ defines the associated Sobolev space of first order; for details on Sobolev spaces, we refer the reader to [1].

For a Hilbert space $V$, the associated inner product and norm are denoted by $(\cdot, \cdot)_{V}$ and $\|\cdot\|_{V} \equiv \sqrt{(\cdot, \cdot)_{V}}$, respectively. A second norm $\|\cdot\|$ on $V$ is called equivalent to $\|\cdot\|_{V}$ if there exist constants $c, C>0$ such that

$$
c\|\cdot\|_{V} \leq\|\cdot\| \leq C\|\cdot\|_{V} .
$$

The dual space of $V$ is given by $V^{\prime}$ and $\langle\cdot, \cdot\rangle \equiv\langle\cdot, \cdot\rangle_{V^{\prime} \times V}$ denotes by default the associated dual pairing. For an element $f \in V^{\prime}$, we consider the dual norm

$$
\|f\|_{V^{\prime}} \equiv \sup _{v \in V} \frac{f(v)}{\|v\|_{V}}
$$

For a bounded linear operator $B: V \rightarrow V^{\prime}, \operatorname{ker}(B)$ and $\operatorname{im}(B)$ refer to its kernel and range, respectively,

$$
\operatorname{ker}(B) \equiv\{v \in V \mid B v=0\}, \quad \operatorname{im}(B) \equiv\left\{f \in V^{\prime} \mid \exists v \in V: B v=f\right\}
$$

moreover, $\operatorname{ker}(B)^{\perp} \equiv\left\{v \in V \mid\left(v, v_{0}\right)_{V}=0, \forall v_{0} \in \operatorname{ker}(B)\right\}$ denotes the orthogonal complement with respect to the inner product $(\cdot, \cdot)_{V}$. 


\section{Appendix B}

\section{Supplementary Comments}

In this section, we provide some additional comments on arguments from functional analysis used in this thesis.

\section{On the Reduced Basis System (2.14)}

We here comment on the steps outlined in $\S 2.3 .2$ in a little more detail. To this end, let $\mu$ be any parameter in $\mathcal{D}$.

We first show that the system $(2.15),(2.16)$ is solvable if and only if $g_{N}(\mu)$ belongs to the range of the operator $B_{N}(\mu)$. It clearly follows from (2.16) that $g_{N}(\mu) \in \operatorname{im}\left(B_{N}(\mu)\right)$ is a necessary condition for (2.15), (2.16) to be solvable. Furthermore, if we assume that $g_{N}(\mu) \in$ $\operatorname{im}\left(B_{N}(\mu)\right)$, we can without loss of generality find an element $u_{N}^{\perp}(\mu) \in \operatorname{ker}\left(B_{N}(\mu)\right)^{\perp}$ such that $B_{N}(\mu) u_{N}^{\perp}(\mu)=g_{N}(\mu)$ in $Y_{N}^{\prime}$. Due to (2.8) and (2.10), it then follows from the classical Lax-Milgram lemma (see, e.g., [89]) that there exists a unique solution $u_{N}^{0}(\mu) \in \operatorname{ker}\left(B_{N}(\mu)\right)$ such that

$$
a\left(u_{N}^{0}(\mu), v_{N} ; \mu\right)=f\left(v_{N} ; \mu\right)-a\left(u_{N}^{\perp}(\mu), v_{N} ; \mu\right), \quad \forall v_{N} \in \operatorname{ker}\left(B_{N}(\mu)\right) .
$$

We now define $u_{N}(\mu) \equiv u_{N}^{0}(\mu)+u_{N}^{\perp}(\mu) \in X_{N}$. Then, $u_{N}(\mu)$ satisfies (2.16) by construction, and it follows from (B.1) that $f_{N}(\mu)-A_{N}(\mu) u_{N}(\mu)$ belongs to the annihilator

$$
\operatorname{ker}\left(B_{N}(\mu)\right)^{0} \equiv\left\{f \in X_{N}^{\prime} \mid f\left(v_{N}\right)=0, \forall v_{N} \in \operatorname{ker}\left(B_{N}(\mu)\right)\right\}
$$

As $X_{N}$ is a discrete space, $\operatorname{ker}\left(B_{N}(\mu)\right)^{0}$ corresponds to the range of the operator $B_{N}(\mu)^{t}$ (see, e.g., [13, §II.1, Proposition 1.2]); thus, we have $f_{N}(\mu)-A_{N}(\mu) u_{N}(\mu) \in \operatorname{im}\left(B_{N}(\mu)^{t}\right)$ and there exists $p_{N}(\mu) \in Y_{N}$ such that $(2.15)$ is satisfied.

It remains to show that the solution $u_{N}(\mu) \in X_{N}$ is unique. For this purpose, let $\left(\bar{u}_{N}(\mu), \bar{p}_{N}(\mu)\right) \in X_{N} \times Y_{N}$ denote a second solution to (2.15), (2.16). It then follows from (2.15) that

$$
a\left(u_{N}(\mu)-\bar{u}_{N}(\mu), v_{N} ; \mu\right)=0, \quad \forall v_{N} \in \operatorname{ker}\left(B_{N}(\mu)\right) .
$$

Moreover, (2.16) provides that $u_{N}(\mu)-\bar{u}_{N}(\mu) \in \operatorname{ker}\left(B_{N}(\mu)\right)$; inserting this above yields $\left\|u_{N}(\mu)-\bar{u}_{N}(\mu)\right\|_{X, \mu}=0$ and we obtain the desired result. 


\section{On the Residual Dual Norm (2.34)}

We here comment on the dual norm of the combined residual $r_{N}(\cdot ; \mu)$ given in $(2.34)$. Let $\mu$ be any parameter in $\mathcal{D}$. As also used in $\S 2.6$, the Riesz representation theorem provides unique elements $\hat{e}_{N}^{1}(\mu) \in X$ and $\hat{e}_{N}^{2}(\mu) \in Y$ such that

$$
\left(\hat{e}_{N}^{1}(\mu), v\right)_{X}=r_{N}^{1}(v ; \mu), \quad \forall v \in X, \quad\left(\hat{e}_{N}^{2}(\mu), q\right)_{Y}=r_{N}^{2}(q ; \mu), \quad \forall q \in Y,
$$

for the residuals defined in (2.32) and (2.33); in particular, the respective dual norms can then be represented as

$$
\left\|r_{N}^{1}(\cdot ; \mu)\right\|_{X^{\prime}}=\left\|\hat{e}_{N}^{1}(\mu)\right\|_{X}, \quad\left\|r_{N}^{2}(\cdot ; \mu)\right\|_{Y^{\prime}}=\left\|\hat{e}_{N}^{2}(\mu)\right\|_{Y} .
$$

Choosing now $\hat{e}_{N}(\mu) \equiv\left(\hat{e}_{N}^{1}(\mu), \hat{e}_{N}^{2}(\mu)\right) \in Z$, we obtain from (B.2) that

$$
\begin{aligned}
\left(\hat{e}_{N}(\mu),(v, q)\right)_{Z} & =\left(\hat{e}_{N}^{1}(\mu), v\right)_{X}+\left(\hat{e}_{N}^{2}(\mu), q\right)_{Y} \\
& =r_{N}^{1}(v ; \mu)+r_{N}^{2}(q ; \mu)=r_{N}((v, q) ; \mu), \quad \forall(v, q) \in Z,
\end{aligned}
$$

and thus $\hat{e}_{N}(\mu) \in Z$ defines the Riesz representer associated with the combined residual $r_{N}(\cdot ; \mu) \in Z^{\prime}$. Consequently,

$$
\left\|r_{N}(\cdot ; \mu)\right\|_{Z^{\prime}}=\left\|\hat{e}_{N}(\mu)\right\|_{Z}=\sqrt{\left\|\hat{e}_{N}^{1}(\mu)\right\|_{X}^{2}+\left\|\hat{e}_{N}^{2}(\mu)\right\|_{Y}^{2}}
$$

and (2.34) follows from (B.3). 


\section{Appendix C}

\section{Re: Chapter 6 - A Crank-Nicolson Method}

For time-integration, we may also employ a Crank-Nicolson method that is often used in practice due to its second-order accuracy (see, e.g., [27, 42, 89, 102]). We here present the corresponding reduced basis methodology for the perturbed problem.

\section{General Problem Statement}

\section{Problem Formulation}

We assume the setting introduced in $\S 6.2 .1$. In addition, for a given $\theta \in[0,1]$, we now denote $f_{\theta}^{k}(\cdot ; \mu) \equiv \theta f^{k}(\cdot ; \mu)+(1-\theta) f^{k-1}(\cdot ; \mu)$ and $g_{\theta}^{k}(\cdot ; \mu) \equiv \theta g^{k}(\cdot ; \mu)+(1-\theta) g^{k-1}(\cdot ; \mu)$ for all $k \in \mathbb{K}$; accordingly, we also set $v_{\theta}^{k} \equiv \theta v^{k}+(1-\theta) v^{k-1}$ and $q_{\theta}^{k} \equiv \theta q^{k}+(1-\theta) q^{k-1}$ for finite sequences $\left(v^{k}\right)_{k \in \mathbb{K}} \subseteq X_{\mathrm{e}}$ and $\left(q^{k}\right)_{k \in \mathbb{K}} \subseteq Y_{\mathrm{e}}$.

Using a Crank-Nicolson method, our "exact" problem now reads as follows: For $\varepsilon>0$ and any given $\mu \in \mathcal{D}$, we find $u_{\mathrm{e}}^{\varepsilon, k}(\mu) \in X_{\mathrm{e}}$ and $p_{\mathrm{e}}^{\varepsilon, k}(\mu) \in Y_{\mathrm{e}}, k \in \mathbb{K}_{0}$, such that $u_{\mathrm{e}}^{\varepsilon, 0}(\mu)=0$ and

$$
\begin{aligned}
& \frac{1}{\Delta t} m\left(u_{\mathrm{e}}^{\varepsilon, k}(\mu)-u_{\mathrm{e}}^{\varepsilon, k-1}(\mu), v ; \mu\right) \\
&+a\left(u_{\mathrm{e}, \theta}^{\varepsilon, k}(\mu), v ; \mu\right)+b\left(v, p_{\mathrm{e}, \theta}^{\varepsilon, k}(\mu) ; \mu\right)=f_{\theta}^{k}(v ; \mu), \forall v \in X_{\mathrm{e}}, k \in \mathbb{K}, \\
& b\left(u_{\mathrm{e}}^{\varepsilon, k}(\mu), q ; \mu\right)-\varepsilon c\left(p_{\mathrm{e}}^{\varepsilon, k}(\mu), q ; \mu\right)=g^{k}(q ; \mu), \quad \forall q \in Y_{\mathrm{e}}, k \in \mathbb{K}_{0},
\end{aligned}
$$

where $\theta=\frac{1}{2}$. Again, as the bilinear form $\frac{1}{\Delta t} m(\cdot, \cdot ; \mu)+\theta a(\cdot, \cdot ; \mu)$ is coercive on $X_{\mathrm{e}}$ for any $\mu \in \mathcal{D}$ from (6.2) and (2.3), the problem (C.1) is uniquely solvable for $\left(u_{\mathrm{e}}^{\varepsilon, k}(\mu), p_{\mathrm{e}}^{\varepsilon, k}(\mu)\right)$, $k \in \mathbb{K}_{0}$, as a saddle point problem according to $\S 4.2 .1$.

\section{Truth Approximation}

Assuming the setting introduced in $\$ 6.2 .2$, our high-fidelity "truth" discretization for (C.1) now reads as follows: For $\varepsilon>0$ and any given $\mu \in \mathcal{D}$, we find $u^{\varepsilon, k}(\mu) \in X$ and $p^{\varepsilon, k}(\mu) \in Y$, $k \in \mathbb{K}_{0}$, such that $u^{\varepsilon, 0}(\mu)=0$ and

$$
\begin{aligned}
& \frac{1}{\Delta t} m\left(u^{\varepsilon, k}(\mu)-u^{\varepsilon, k-1}(\mu), v ; \mu\right) \\
& +a\left(u_{\theta}^{\varepsilon, k}(\mu), v ; \mu\right)+b\left(v, p_{\theta}^{\varepsilon, k}(\mu) ; \mu\right)=f_{\theta}^{k}(v ; \mu), \quad \forall v \in X, k \in \mathbb{K}, \\
& b\left(u^{\varepsilon, k}(\mu), q ; \mu\right)-\varepsilon c\left(p^{\varepsilon, k}(\mu), q ; \mu\right)=g^{k}(q ; \mu), \quad \forall q \in Y, k \in \mathbb{K}_{0},
\end{aligned}
$$


where $\theta=\frac{1}{2}$. Analogous to the exact problem, the problem (C.2) is uniquely solvable for $\left(u^{\varepsilon, k}(\mu), p^{\varepsilon, k}(\mu)\right), k \in \mathbb{K}_{0}$, according to $\S 4.2 .2$. The system can again be equivalently written in operator notation,

$$
\begin{aligned}
M(\mu) \frac{u^{\varepsilon, k}(\mu)-u^{\varepsilon, k-1}(\mu)}{\Delta t}+A(\mu) u_{\theta}^{\varepsilon, k}(\mu)+B(\mu)^{t} p_{\theta}^{\varepsilon, k}(\mu)=f_{\theta}^{k}(\mu) & \text { in } X^{\prime}, k \in \mathbb{K}, \\
B(\mu) u^{\varepsilon, k}(\mu)-\varepsilon C(\mu) p^{\varepsilon, k}(\mu)=g^{k}(\mu) & \text { in } Y^{\prime}, k \in \mathbb{K}_{0},
\end{aligned}
$$

where $\left.f_{\theta}^{k}(\mu) \equiv f_{\theta}^{k}(\cdot ; \mu)\right|_{X} \in X^{\prime}$ for all $k \in \mathbb{K}$.

\section{Reduced Basis Approximation}

Suppose that we are given a set of nested, low-dimensional reduced basis approximation subspaces $X_{N} \subset X_{N+1} \subset X$ and $Y_{N} \subset Y_{N+1} \subset Y, N \in \mathbb{N}_{\max } \equiv\left\{1, \ldots, N_{\max }\right\}$, then the reduced basis approximation is defined as the Galerkin projection with respect to the truth problem (C.2) onto these low-dimensional subspaces: For $\varepsilon>0$ and any given $\mu \in \mathcal{D}$, we find $u_{N}^{\varepsilon, k}(\mu) \in X_{N}$ and $p_{N}^{\varepsilon, k}(\mu) \in Y_{N}, k \in \mathbb{K}_{0}$, such that $u_{N}^{\varepsilon, 0}(\mu)=0$ and

$$
\begin{aligned}
& \frac{1}{\Delta t} m\left(u_{N}^{\varepsilon, k}(\mu)-u_{N}^{\varepsilon, k-1}(\mu), v_{N} ; \mu\right) \\
&+a\left(u_{N, \theta}^{\varepsilon, k}(\mu), v_{N} ; \mu\right)+b\left(v_{N}, p_{N, \theta}^{\varepsilon, k}(\mu) ; \mu\right)=f_{\theta}^{k}\left(v_{N} ; \mu\right), \forall v_{N} \in X_{N}, k \in \mathbb{K}, \\
& b\left(u_{N}^{\varepsilon, k}(\mu), q_{N} ; \mu\right)-\varepsilon c\left(p_{N}^{\varepsilon, k}(\mu), q_{N} ; \mu\right)=g^{k}\left(q_{N} ; \mu\right), \quad \forall q_{N} \in Y_{N}, k \in \mathbb{K}_{0},
\end{aligned}
$$

where $\theta=\frac{1}{2}$. The problem is uniquely solvable for $\left(u_{N}^{\varepsilon, k}(\mu), p_{N}^{\varepsilon, k}(\mu)\right), k \in \mathbb{K}_{0}$, for any choice of $X_{N}, Y_{N}$ (see $\S 4.3 .2$ ). Written in operator notation, it also reads

$$
\begin{aligned}
\frac{1}{\Delta t} M_{N}(\mu)\left(u_{N}^{\varepsilon, k}(\mu)-u_{N}^{\varepsilon, k-1}(\mu)\right) & \\
+A_{N}(\mu) u_{N, \theta}^{\varepsilon, k}(\mu)+B_{N}(\mu)^{t} p_{N, \theta}^{\varepsilon, k}(\mu) & =f_{N, \theta}^{k}(\mu) \quad \text { in } X_{N}^{\prime}, k \in \mathbb{K}, \\
B_{N}(\mu) u_{N}^{\varepsilon, k}(\mu)-\varepsilon C_{N}(\mu) p_{N}^{\varepsilon, k}(\mu)=g_{N}^{k}(\mu) & \text { in } Y_{N}^{\prime}, k \in \mathbb{K}_{0},
\end{aligned}
$$

where $A_{N}(\mu), B_{N}(\mu), B_{N}(\mu)^{t}, C_{N}(\mu), M_{N}(\mu)$, and $g_{N}^{k}(\mu)$ are defined as in $\S 6.3$ and $\left.f_{N, \theta}^{k}(\mu) \equiv f_{\theta}^{k}(\cdot ; \mu)\right|_{X_{N}} \in X_{N}^{\prime}$ for all $k \in \mathbb{K}$.

\section{A Posteriori Error Estimation}

We now develop upper bounds for the errors in our reduced basis approximations that are rigorous, sharp, and computationally efficient. As before, the errors shall be measured relative to the respective truth approximations.

For $\mu \in \mathcal{D}$, we consider the errors

$$
\begin{aligned}
& e_{N}^{u}(\mu) \equiv\left(e_{N}^{u, k}(\mu)\right)_{k \in \mathbb{K}}, \text { where } e_{N}^{u, k}(\mu) \equiv u^{\varepsilon, k}(\mu)-u_{N}^{\varepsilon, k}(\mu) \in X, k \in \mathbb{K}_{0}, \\
& e_{N}^{p}(\mu) \equiv\left(e_{N}^{p, k}(\mu)\right)_{k \in \mathbb{K}}, \text { where } e_{N}^{p, k}(\mu) \equiv p^{\varepsilon, k}(\mu)-p_{N}^{\varepsilon, k}(\mu) \in Y, k \in \mathbb{K}_{0}, \\
& e_{N}^{\varepsilon}(\mu) \equiv\left(e_{N}^{\varepsilon, k}(\mu)\right)_{k \in \mathbb{K}}, \text { where } e_{N}^{\varepsilon, k}(\mu) \equiv\left(e_{N}^{u, k}(\mu), e_{N}^{p, k}(\mu)\right) \in Z, k \in \mathbb{K}_{0},
\end{aligned}
$$


in the reduced basis approximations $\left(u_{N}^{\varepsilon, k}(\mu), p_{N}^{\varepsilon, k}(\mu)\right), k \in \mathbb{K}_{0}$, with respect to the truth solution $\left(u^{\varepsilon, k}(\mu), p^{\varepsilon, k}(\mu)\right), k \in \mathbb{K}_{0}$; we note that in particular $e_{N}^{u, 0}(\mu)=0$ from our initial conditions. The error in the reduced basis approximation shall then be measured in the "spatio-temporal" energy norm

$$
\left\|\left(v^{j}, q^{j}\right)_{j \in \mathbb{K}}\right\|_{\ell^{2}(0, k ; Z)} \equiv\left(\left\|v^{k}\right\|_{\mu}^{2}+\Delta t \sum_{j=1}^{k}\left\|v_{\theta}^{j}\right\|_{X, \mu}^{2}+\varepsilon\left\|q_{\theta}^{j}\right\|_{Y, \mu}^{2}\right)^{1 / 2},
$$

where $\left(v^{j}, q^{j}\right)_{j \in \mathbb{K}} \subseteq Z, k \in \mathbb{K}$.

To formulate our reduced basis a posteriori error bounds, we again need several ingredients: We rely on (Online-)efficient lower (and upper) bounds (4.29) to the truth coercivity constants (2.10) and (4.12) as well as on the residuals associated with the reduced basis approximation $\left(u_{N}^{\varepsilon, k}(\mu), p_{N}^{\varepsilon, k}(\mu)\right), k \in \mathbb{K}_{0}$,

$$
\begin{aligned}
& r_{N, \theta}^{1, k}(\cdot ; \mu) \equiv f_{\theta}^{k}(\mu)-M(\mu) \frac{u_{N}^{\varepsilon, k}(\mu)-u_{N}^{\varepsilon, k-1}(\mu)}{\Delta t}-A(\mu) u_{N, \theta}^{\varepsilon, k}(\mu)-B(\mu)^{t} p_{N, \theta}^{\varepsilon, k}(\mu) \in X^{\prime} \\
& r_{N, \theta}^{2, k}(\cdot ; \mu) \equiv g_{\theta}^{k}(\mu)-B(\mu) u_{N, \theta}^{\varepsilon, k}(\mu)+\varepsilon C(\mu) p_{N, \theta}^{\varepsilon, k}(\mu) \in Y^{\prime}
\end{aligned}
$$

where $\left.g_{\theta}^{k}(\mu) \equiv g_{\theta}^{k}(\cdot ; \mu)\right|_{X} \in X^{\prime}$ for all $k \in \mathbb{K}$ and $\mu \in \mathcal{D}$.

We can now state the following result.

Proposition C.0.1. For any given $\mu \in \mathcal{D}, N \in \mathbb{N}_{\max }, k \in \mathbb{K}$, and $\alpha_{a}^{\mathrm{LB}}(\mu), \alpha_{c}^{\mathrm{LB}}(\mu)$ satisfying (4.29), we define

$$
\Delta_{N}^{\varepsilon, k}(\mu) \equiv\left(\Delta t \sum_{j=1}^{k} \frac{\left\|r_{N, \theta}^{1, j}(\cdot ; \mu)\right\|_{X^{\prime}}^{2}}{\alpha_{a}^{\mathrm{LB}}(\mu)}+\frac{\left\|r_{N, \theta}^{2, j}(\cdot ; \mu)\right\|_{Y^{\prime}}^{2}}{\varepsilon \alpha_{c}^{\mathrm{LB}}(\mu)}\right)^{1 / 2} .
$$

Then, $\Delta_{N}^{\varepsilon, k}(\mu)$ represents an upper bound for the error $e_{N}^{\varepsilon}(\mu)$ measured in the "spatiotemporal" energy norm (C.6),

$$
\left\|e_{N}^{\varepsilon}(\mu)\right\|_{\ell^{2}(0, k ; Z)} \leq \Delta_{N}^{\varepsilon, k}(\mu), \quad \forall k \in \mathbb{K}, \mu \in \mathcal{D}, N \in \mathbb{N}_{\max }
$$

Proof. Let $\mu$ be any parameter in $\mathcal{D}, N \in \mathbb{N}_{\max }$, and $k \in \mathbb{K}$. For clarity of exposition, we suppress the argument $\mu$ in this proof.

Take any $1 \leq j \leq k$. From (C.7), (C.8), and (C.2), the errors satisfy the equations

$$
\begin{aligned}
\frac{1}{\Delta t} m\left(e_{N}^{u, j}-e_{N}^{u, j-1}, v\right)+a\left(e_{N, \theta}^{u, j}, v\right)+b\left(v, e_{N, \theta}^{p, j}\right) & =r_{N, \theta}^{1, j}(v), \quad \forall v \in X, \\
b\left(e_{N, \theta}^{u, j}, q\right)-\varepsilon c\left(e_{N, \theta}^{p, j}, q\right) & =r_{N, \theta}^{2, j}(q), \quad \forall q \in Y .
\end{aligned}
$$

Setting here $v=e_{N, \theta}^{u, j}, q=e_{N, \theta}^{p, j}$ and subtracting the second from the first equation, we obtain

$$
\begin{array}{r}
\frac{1}{\Delta t} m\left(e_{N}^{u, j}-e_{N}^{u, j-1}, e_{N, \theta}^{u, j}\right)+\left\|e_{N, \theta}^{u, j}\right\|_{X, \mu}^{2}+\varepsilon\left\|e_{N, \theta}^{p, j}\right\|_{Y, \mu}^{2}=r_{N, \theta}^{1, j}\left(e_{N, \theta}^{u, j}\right)-r_{N, \theta}^{2, j}\left(e_{N, \theta}^{p, j}\right) \\
\leq\left\|r_{N, \theta}^{1, j}\right\|_{X^{\prime}}\left\|e_{N, \theta}^{u, j}\right\|_{X}+\left\|r_{N, \theta}^{2, j}\right\|_{Y^{\prime}}\left\|e_{N, \theta}^{p, j}\right\|_{Y} .
\end{array}
$$


On the right-hand side, we may now apply (2.10), (4.12), and Young's inequality such that

$$
\begin{aligned}
& \frac{1}{\Delta t} m\left(e_{N}^{u, j}-e_{N}^{u, j-1}, e_{N, \theta}^{u, j}\right)+\left\|e_{N, \theta}^{u, j}\right\|_{X, \mu}^{2}+\varepsilon\left\|e_{N, \theta}^{p, j}\right\|_{Y, \mu}^{2} \\
& \leq \frac{\left\|r_{N, \theta}^{1, j}\right\|_{X^{\prime}}}{\sqrt{\alpha_{a}}}\left\|e_{N, \theta}^{u, j}\right\|_{X, \mu}+\frac{\left\|r_{N, \theta}^{2, j}\right\|_{Y^{\prime}}}{\sqrt{\alpha_{c}}}\left\|e_{N, \theta}^{p, j}\right\|_{Y, \mu} \\
& \quad \leq \frac{1}{2}\left(\frac{\left\|r_{N, \theta}^{1, j}\right\|_{X^{\prime}}^{2}}{\alpha_{a}}+\left\|e_{N, \theta}^{u, j}\right\|_{X, \mu}^{2}+\frac{\left\|r_{N, \theta}^{2, j}\right\|_{Y^{\prime}}^{2}}{\varepsilon \alpha_{c}}+\varepsilon\left\|e_{N, \theta}^{p, j}\right\|_{Y, \mu}^{2}\right) ;
\end{aligned}
$$

rearranging terms then yields

$$
\frac{2}{\Delta t} m\left(e_{N}^{u, j}-e_{N}^{u, j-1}, e_{N, \theta}^{u, j}\right)+\left\|e_{N, \theta}^{u, j}\right\|_{X, \mu}^{2}+\varepsilon\left\|e_{N, \theta}^{p, j}\right\|_{Y, \mu}^{2} \leq \frac{\left\|r_{N, \theta}^{1, j}\right\|_{X^{\prime}}^{2}}{\alpha_{a}}+\frac{\left\|r_{N, \theta}^{2, j}\right\|_{Y^{\prime}}^{2}}{\varepsilon \alpha_{c}} .
$$

As in particular $\theta=\frac{1}{2}$, the mass term on the left-hand side directly reads

$$
\frac{1}{\Delta t}\left(\left\|e_{N}^{u, j}\right\|_{\mu}^{2}-\left\|e_{N}^{u, j-1}\right\|_{\mu}^{2}\right)+\left\|e_{N, \theta}^{u, j}\right\|_{X, \mu}^{2}+\varepsilon\left\|e_{N, \theta}^{p, j}\right\|_{Y, \mu}^{2} \leq \frac{\left\|r_{N, \theta}^{1, j}\right\|_{X^{\prime}}^{2}}{\alpha_{a}}+\frac{\left\|r_{N, \theta}^{2, j}\right\|_{Y^{\prime}}^{2}}{\varepsilon \alpha_{c}}
$$

and the statement follows from applying the sum $\sum_{j=1}^{k}, e_{N}^{u, 0}=0$, and (4.29).

\section{Numerical Results}

We now apply the developed reduced basis methodology to the model problem described in $\S 6.7$. We set $T=1$ and consider a constant time step size $\Delta t$ corresponding to $K=100$ time levels. The truth discretization is based on a fine mesh with a total of $\mathcal{N}=72,076$ velocity and pressure degrees of freedom. In this section, all numerical results are attained using the open source software rb00mit [65], an implementation of the reduced basis framework within the $\mathrm{C}++$ parallel finite element library libMesh [62].

To build our low-dimensional reduced basis approximation spaces $X_{N}, Y_{N}, N \in \mathbb{N}_{\max }$, we again apply the POD greedy procedure described in Algorithm 6 (see §6.6). The sampling process is based on an exhaustive random sample $\Sigma \subset \mathcal{D}$ of size $|\Sigma|=4,900$, $\Delta N=2, \delta_{\text {tol }}^{\kappa}=10^{3}$, and the relative reduced basis a posteriori error bound $\Delta_{N}(\mu)=$ $\Delta_{N}^{\varepsilon, K}(\mu) /\left\|\left(u_{N}^{\varepsilon, j}(\mu)\right)_{j \in \mathbb{K}}\right\|_{\ell^{2}(0, K ; Z)}($ see $($ C.6), (C.9)).

Using a Crank-Nicolson method, numerical results illustrate the same effects as observed from a backward Euler method; we therefore present the following data without further comments at this point and refer the reader to $\S 6.8$ and $\S 6.9$ for their evaluation.

Computation times for the reduced basis approximations $\left(u_{N}^{\varepsilon, k}(\mu), p_{N}^{\varepsilon, k}(\mu)\right), k \in \mathbb{K}$, and associated a posteriori error bounds $\Delta_{N}^{\varepsilon, k}(\mu), k \in \mathbb{K}$, are given in Table C.1; for comparison, once the $\mu$-independent parts in (2.7) have been formed, direct computation of the truth approximation $\left(u^{\varepsilon, k}(\mu), p^{\varepsilon, k}(\mu)\right), k \in \mathbb{K}$, (i.e., assembly and solution of (C.2)) requires roughly 23 seconds on a $2.66 \mathrm{GHz}$ Intel Core 2 Duo processor. 

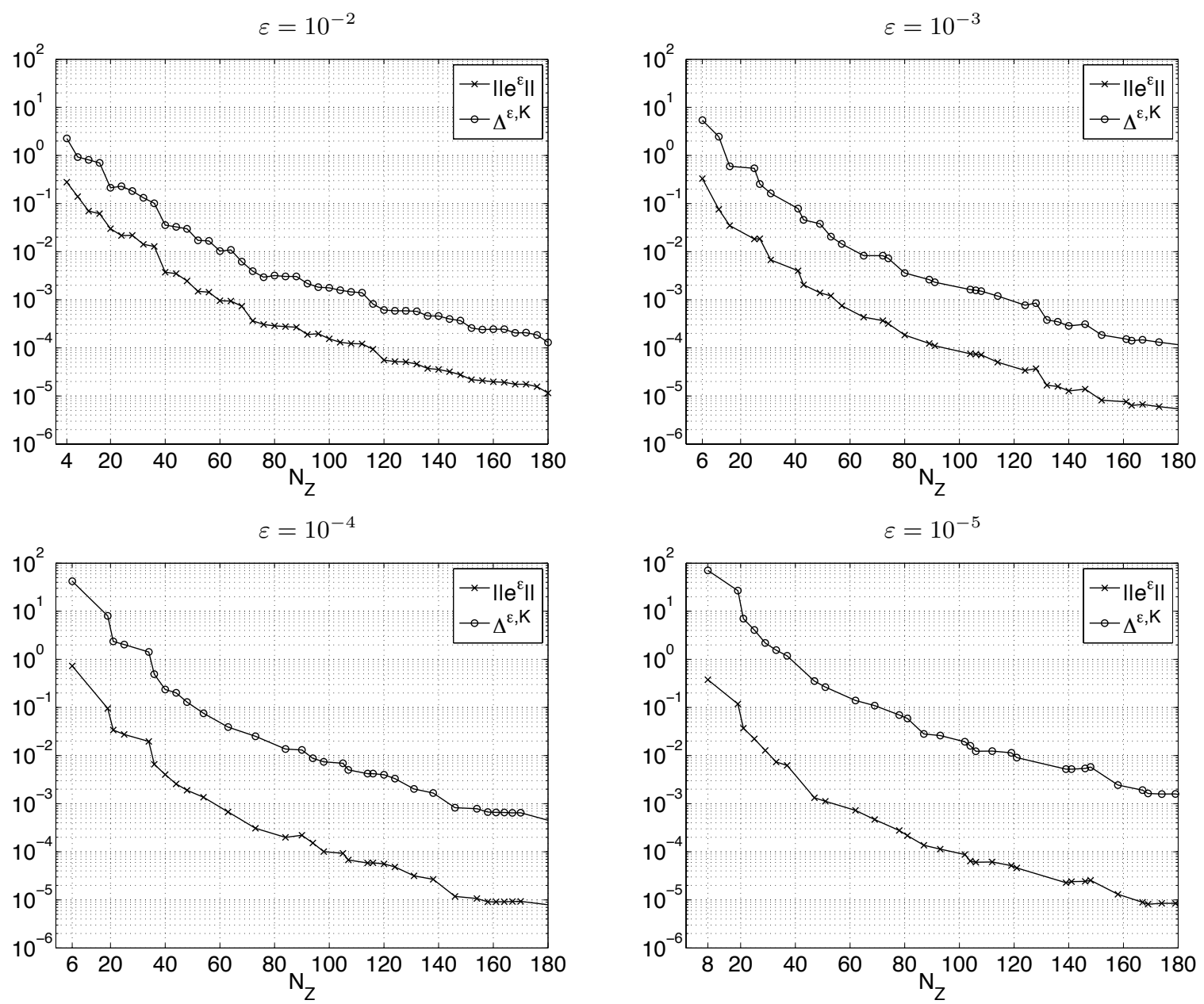

Figure C.1.: Maximum error $\left\|e_{N}^{\varepsilon}(\mu)\right\|_{\ell^{2}(0, K ; Z)}$ (see (C.5), (C.6)) and maximum error bound $\Delta_{N}^{\varepsilon, K}(\mu)$ (see (C.9)) normalized with respect to $\left\|\left(u^{\varepsilon, j}(\mu), p^{\varepsilon, j}(\mu)\right)_{j \in \mathbb{K}}\right\|_{\ell^{2}(0, K ; Z)}$ shown as functions of $N_{Z}$ for different values of $\varepsilon$; the maximum is taken over 25 parameter values.

\begin{tabular}{c|c|c|c|c|c}
\hline$\varepsilon$ & $N_{Z}$ & $N$ & $\left(u_{N}^{\varepsilon, k}(\mu), p_{N}^{\varepsilon, k}(\mu)\right), k \in \mathbb{K}$ & $\Delta_{N}^{\varepsilon, k}(\mu), k \in \mathbb{K}$ & Total \\
\hline $10^{-2}$ & $68(116)$ & $17(29)$ & $3.71(8.11)$ & $14.55(28.39)$ & $18.26(36.50)$ \\
\hline $10^{-3}$ & $65(124)$ & $12(22)$ & $3.60(9.46)$ & $15.64(34.74)$ & $19.24(44.20)$ \\
\hline $10^{-4}$ & $94(146)$ & $15(25)$ & $6.30(13.03)$ & $25.06(43.07)$ & $31.37(56.10)$ \\
\hline $10^{-5}$ & $121(202)$ & $21(33)$ & $9.19(26.62)$ & $33.94(69.19)$ & $43.13(95.81)$ \\
\hline
\end{tabular}

Table C.1.: Average computation times in milliseconds for the Online evaluation of $\left(u_{N}^{\varepsilon, k}(\mu), p_{N}^{\varepsilon, k}(\mu)\right), k \in \mathbb{K}$, (assembly and solution of (C.3)) and the error bounds $\Delta_{N}^{\varepsilon, k}(\mu), k \in \mathbb{K}$, (see (C.9)) for different values of $\varepsilon$ with a prescribed accuracy of at least $1 \%$ (resp., 0.1\%) for the reduced basis approximations $\left(u_{N}^{\varepsilon, k}(\mu), p_{N}^{\varepsilon, k}(\mu)\right), k \in \mathbb{K}$. 
(a) $\varepsilon=10^{-2}$

(b) $\varepsilon=10^{-3}$

(c) $\varepsilon=10^{-4}$

(d) $\varepsilon=10^{-5}$
$N=17\left(N_{Z}=68\right)$

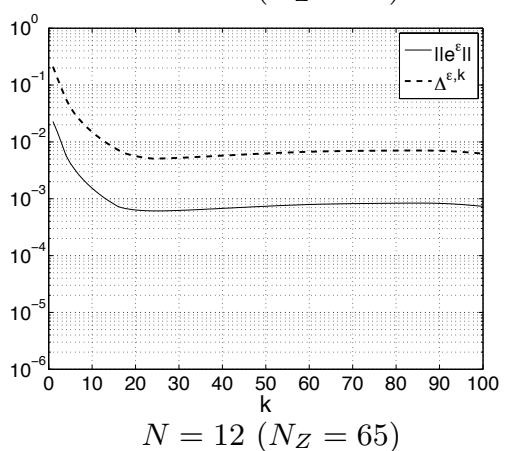

$N=12\left(N_{Z}=65\right)$

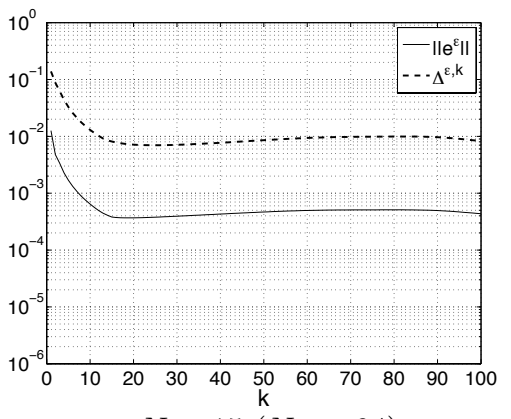

$N=15\left(N_{Z}=94\right)$

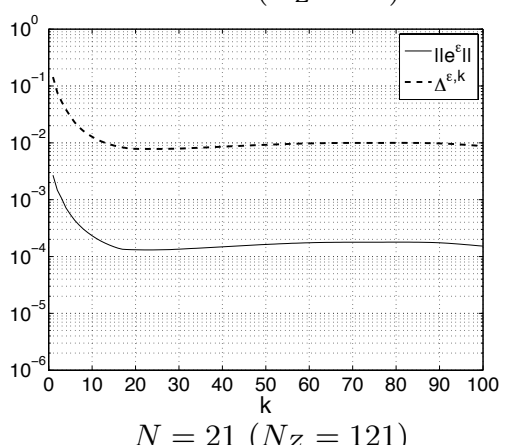

$N=21\left(N_{Z}=121\right)$

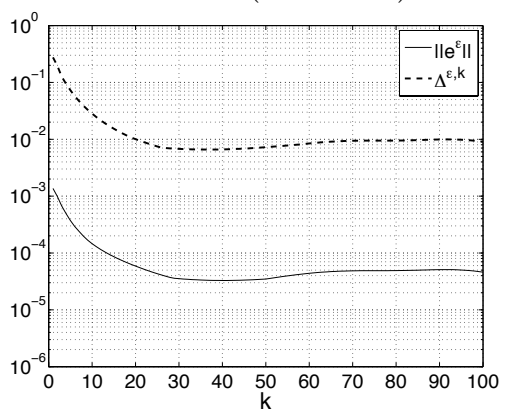

$N=29\left(N_{Z}=116\right)$

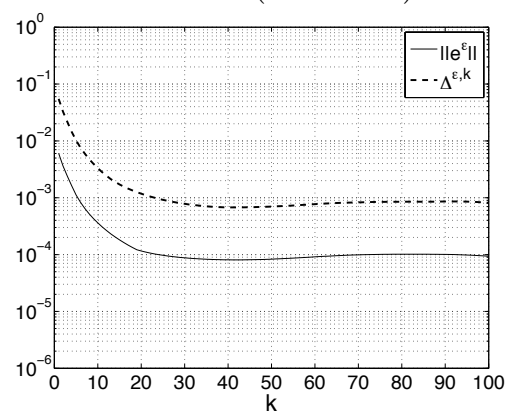

$N=22\left(N_{Z}=124\right)$

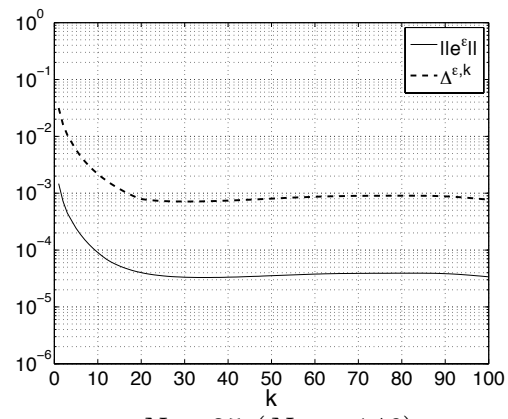

$N=25\left(N_{Z}=146\right)$
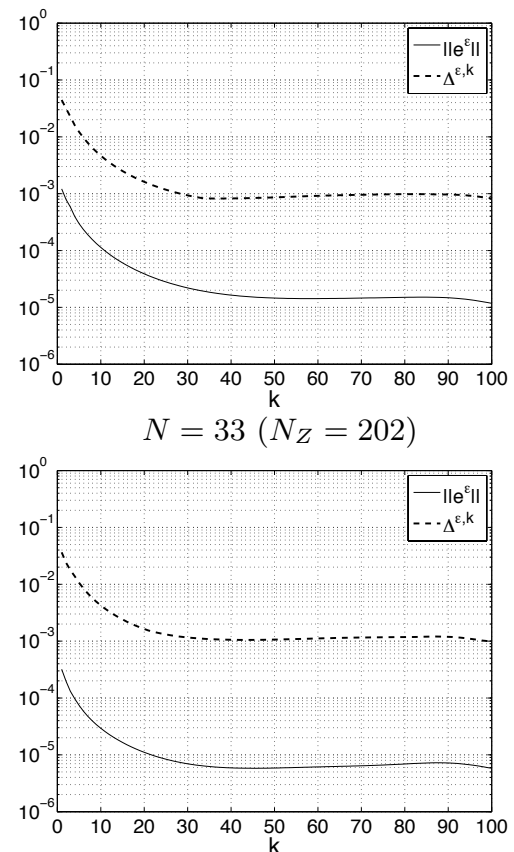

Figure C.2.: Maximum error $\left\|e_{N}^{\varepsilon}(\mu)\right\|_{\ell^{2}(0, k ; Z)}$ (see (C.5), (C.6)) and maximum error bound $\Delta_{N}^{\varepsilon, k}(\mu)$ (see (C.9)) normalized with respect to $\left\|\left(u^{\varepsilon, j}(\mu), p^{\varepsilon, j}(\mu)\right)_{j \in \mathbb{K}}\right\|_{\ell^{2}(0, k ; Z)}$ shown as functions of $k \in \mathbb{K}$ for several values of $N$ for (a) $\varepsilon=10^{-2}$, (b) $\varepsilon=$ $10^{-3}$, (c) $\varepsilon=10^{-4}$, and (d) $\varepsilon=10^{-5}$; the maximum is taken over 25 parameter values. 
(a) $\varepsilon=10^{-2}$

\begin{tabular}{c|c|c|c|c|c|c|c}
\hline$N$ & $N_{Z}$ & $k=10$ & $k=20$ & $k=40$ & $k=60$ & $k=80$ & $k=100$ \\
\hline 5 & 20 & $1.131 \cdot 10^{1}$ & $1.211 \cdot 10^{1}$ & $1.259 \cdot 10^{1}$ & $1.268 \cdot 10^{1}$ & $1.267 \cdot 10^{1}$ & $1.252 \cdot 10^{1}$ \\
10 & 40 & $1.115 \cdot 10^{1}$ & $1.111 \cdot 10^{1}$ & $1.133 \cdot 10^{1}$ & $1.131 \cdot 10^{1}$ & $1.126 \cdot 10^{1}$ & $1.128 \cdot 10^{1}$ \\
15 & 60 & $1.123 \cdot 10^{1}$ & $1.168 \cdot 10^{1}$ & $1.247 \cdot 10^{1}$ & $1.253 \cdot 10^{1}$ & $1.250 \cdot 10^{1}$ & $1.248 \cdot 10^{1}$ \\
20 & 80 & $1.025 \cdot 10^{1}$ & $1.056 \cdot 10^{1}$ & $1.152 \cdot 10^{1}$ & $1.193 \cdot 10^{1}$ & $1.186 \cdot 10^{1}$ & $1.180 \cdot 10^{1}$ \\
25 & 100 & $1.260 \cdot 10^{1}$ & $1.224 \cdot 10^{1}$ & $1.154 \cdot 10^{1}$ & $1.160 \cdot 10^{1}$ & $1.162 \cdot 10^{1}$ & $1.149 \cdot 10^{1}$ \\
30 & 120 & $1.135 \cdot 10^{1}$ & $1.175 \cdot 10^{1}$ & $1.193 \cdot 10^{1}$ & $1.260 \cdot 10^{1}$ & $1.232 \cdot 10^{1}$ & $1.215 \cdot 10^{1}$ \\
35 & 140 & $1.139 \cdot 10^{1}$ & $1.193 \cdot 10^{1}$ & $1.248 \cdot 10^{1}$ & $1.274 \cdot 10^{1}$ & $1.283 \cdot 10^{1}$ & $1.294 \cdot 10^{1}$ \\
40 & 160 & $1.135 \cdot 10^{1}$ & $1.133 \cdot 10^{1}$ & $1.210 \cdot 10^{1}$ & $1.261 \cdot 10^{1}$ & $1.259 \cdot 10^{1}$ & $1.244 \cdot 10^{1}$ \\
45 & 180 & $1.137 \cdot 10^{1}$ & $1.145 \cdot 10^{1}$ & $1.150 \cdot 10^{1}$ & $1.190 \cdot 10^{1}$ & $1.203 \cdot 10^{1}$ & $1.224 \cdot 10^{1}$ \\
\hline
\end{tabular}

(b) $\varepsilon=10^{-3}$

\begin{tabular}{c|c|c|c|c|c|c|c}
\hline$N$ & $N_{Z}$ & $k=10$ & $k=20$ & $k=40$ & $k=60$ & $k=80$ & $k=100$ \\
\hline 3 & 16 & $2.432 \cdot 10^{1}$ & $3.144 \cdot 10^{1}$ & $3.393 \cdot 10^{1}$ & $3.479 \cdot 10^{1}$ & $3.442 \cdot 10^{1}$ & $3.365 \cdot 10^{1}$ \\
7 & 41 & $3.403 \cdot 10^{1}$ & $3.606 \cdot 10^{1}$ & $3.809 \cdot 10^{1}$ & $4.015 \cdot 10^{1}$ & $3.835 \cdot 10^{1}$ & $3.970 \cdot 10^{1}$ \\
11 & 57 & $2.316 \cdot 10^{1}$ & $2.354 \cdot 10^{1}$ & $2.715 \cdot 10^{1}$ & $2.868 \cdot 10^{1}$ & $2.881 \cdot 10^{1}$ & $2.810 \cdot 10^{1}$ \\
15 & 80 & $2.243 \cdot 10^{1}$ & $2.583 \cdot 10^{1}$ & $3.045 \cdot 10^{1}$ & $3.177 \cdot 10^{1}$ & $3.209 \cdot 10^{1}$ & $3.186 \cdot 10^{1}$ \\
18 & 104 & $2.484 \cdot 10^{1}$ & $2.543 \cdot 10^{1}$ & $2.758 \cdot 10^{1}$ & $2.794 \cdot 10^{1}$ & $2.741 \cdot 10^{1}$ & $2.816 \cdot 10^{1}$ \\
22 & 124 & $2.409 \cdot 10^{1}$ & $2.414 \cdot 10^{1}$ & $2.538 \cdot 10^{1}$ & $2.791 \cdot 10^{1}$ & $2.730 \cdot 10^{1}$ & $2.703 \cdot 10^{1}$ \\
26 & 140 & $2.526 \cdot 10^{1}$ & $2.538 \cdot 10^{1}$ & $2.589 \cdot 10^{1}$ & $2.587 \cdot 10^{1}$ & $2.542 \cdot 10^{1}$ & $2.591 \cdot 10^{1}$ \\
29 & 161 & $2.392 \cdot 10^{1}$ & $2.412 \cdot 10^{1}$ & $2.530 \cdot 10^{1}$ & $2.562 \cdot 10^{1}$ & $2.548 \cdot 10^{1}$ & $2.614 \cdot 10^{1}$ \\
33 & 184 & $2.574 \cdot 10^{1}$ & $2.580 \cdot 10^{1}$ & $2.696 \cdot 10^{1}$ & $2.790 \cdot 10^{1}$ & $2.741 \cdot 10^{1}$ & $2.727 \cdot 10^{1}$ \\
\hline
\end{tabular}

(c) $\varepsilon=10^{-4}$

\begin{tabular}{c|c|c|c|c|c|c|c}
\hline$N$ & $N_{Z}$ & $k=10$ & $k=20$ & $k=40$ & $k=60$ & $k=80$ & $k=100$ \\
\hline 2 & 19 & $1.112 \cdot 10^{2}$ & $1.250 \cdot 10^{2}$ & $1.226 \cdot 10^{2}$ & $1.280 \cdot 10^{2}$ & $1.300 \cdot 10^{2}$ & $1.273 \cdot 10^{2}$ \\
7 & 40 & $9.345 \cdot 10^{1}$ & $9.363 \cdot 10^{1}$ & $1.176 \cdot 10^{2}$ & $1.191 \cdot 10^{2}$ & $1.167 \cdot 10^{2}$ & $1.123 \cdot 10^{2}$ \\
11 & 63 & $7.780 \cdot 10^{1}$ & $7.809 \cdot 10^{1}$ & $8.898 \cdot 10^{1}$ & $8.997 \cdot 10^{1}$ & $9.095 \cdot 10^{1}$ & $9.200 \cdot 10^{1}$ \\
13 & 84 & $9.159 \cdot 10^{1}$ & $9.247 \cdot 10^{1}$ & $9.431 \cdot 10^{1}$ & $9.236 \cdot 10^{1}$ & $9.097 \cdot 10^{1}$ & $9.132 \cdot 10^{1}$ \\
16 & 98 & $7.357 \cdot 10^{1}$ & $7.571 \cdot 10^{1}$ & $1.034 \cdot 10^{2}$ & $1.074 \cdot 10^{2}$ & $1.067 \cdot 10^{2}$ & $1.044 \cdot 10^{2}$ \\
21 & 120 & $6.504 \cdot 10^{1}$ & $7.179 \cdot 10^{1}$ & $8.352 \cdot 10^{1}$ & $8.453 \cdot 10^{1}$ & $8.345 \cdot 10^{1}$ & $8.272 \cdot 10^{1}$ \\
24 & 138 & $6.587 \cdot 10^{1}$ & $6.658 \cdot 10^{1}$ & $7.325 \cdot 10^{1}$ & $7.924 \cdot 10^{1}$ & $8.110 \cdot 10^{1}$ & $8.037 \cdot 10^{1}$ \\
28 & 161 & $6.715 \cdot 10^{1}$ & $6.829 \cdot 10^{1}$ & $7.325 \cdot 10^{1}$ & $8.367 \cdot 10^{1}$ & $8.448 \cdot 10^{1}$ & $8.577 \cdot 10^{1}$ \\
32 & 181 & $7.303 \cdot 10^{1}$ & $7.364 \cdot 10^{1}$ & $7.573 \cdot 10^{1}$ & $8.103 \cdot 10^{1}$ & $8.291 \cdot 10^{1}$ & $8.372 \cdot 10^{1}$ \\
\hline
\end{tabular}

(d) $\varepsilon=10^{-5}$

\begin{tabular}{c|c|c|c|c|c|c|c}
\hline$N$ & $N_{Z}$ & $k=10$ & $k=20$ & $k=40$ & $k=60$ & $k=80$ & $k=100$ \\
\hline 2 & 19 & $3.584 \cdot 10^{2}$ & $4.002 \cdot 10^{2}$ & $3.782 \cdot 10^{2}$ & $3.938 \cdot 10^{2}$ & $4.090 \cdot 10^{2}$ & $4.029 \cdot 10^{2}$ \\
7 & 37 & $2.557 \cdot 10^{2}$ & $3.281 \cdot 10^{2}$ & $3.393 \cdot 10^{2}$ & $3.369 \cdot 10^{2}$ & $3.348 \cdot 10^{2}$ & $3.335 \cdot 10^{2}$ \\
10 & 62 & $2.730 \cdot 10^{2}$ & $2.891 \cdot 10^{2}$ & $3.212 \cdot 10^{2}$ & $3.096 \cdot 10^{2}$ & $3.087 \cdot 10^{2}$ & $3.165 \cdot 10^{2}$ \\
13 & 81 & $2.729 \cdot 10^{2}$ & $3.452 \cdot 10^{2}$ & $3.700 \cdot 10^{2}$ & $3.714 \cdot 10^{2}$ & $3.709 \cdot 10^{2}$ & $3.730 \cdot 10^{2}$ \\
16 & 102 & $2.616 \cdot 10^{2}$ & $3.130 \cdot 10^{2}$ & $3.189 \cdot 10^{2}$ & $3.135 \cdot 10^{2}$ & $3.124 \cdot 10^{2}$ & $3.202 \cdot 10^{2}$ \\
21 & 121 & $2.092 \cdot 10^{2}$ & $2.249 \cdot 10^{2}$ & $2.791 \cdot 10^{2}$ & $2.969 \cdot 10^{2}$ & $2.976 \cdot 10^{2}$ & $3.033 \cdot 10^{2}$ \\
23 & 141 & $2.572 \cdot 10^{2}$ & $2.785 \cdot 10^{2}$ & $3.404 \cdot 10^{2}$ & $3.487 \cdot 10^{2}$ & $3.485 \cdot 10^{2}$ & $3.466 \cdot 10^{2}$ \\
26 & 158 & $2.168 \cdot 10^{2}$ & $2.240 \cdot 10^{2}$ & $2.477 \cdot 10^{2}$ & $2.531 \cdot 10^{2}$ & $2.594 \cdot 10^{2}$ & $2.657 \cdot 10^{2}$ \\
31 & 181 & $2.479 \cdot 10^{2}$ & $2.476 \cdot 10^{2}$ & $2.641 \cdot 10^{2}$ & $2.757 \cdot 10^{2}$ & $2.739 \cdot 10^{2}$ & $2.951 \cdot 10^{2}$ \\
\hline
\end{tabular}

Table C.2.: Maximum effectivities $\eta_{N}^{\varepsilon, k}(\mu) \equiv \Delta_{N}^{\varepsilon, k}(\mu) /\left\|e_{N}^{\varepsilon}(\mu)\right\|_{\ell^{2}(0, k ; Z)}$ (see (C.10)) for several values of $k \in \mathbb{K}$ and $N$ for (a) $\varepsilon=10^{-2}$, (b) $\varepsilon=10^{-3}$, (c) $\varepsilon=10^{-4}$, and (d) $\varepsilon=10^{-5}$; the maximum is taken over 25 parameter values. 



\title{
Curriculum Vitae
}

\author{
Name: Gerner \\ First name: Anna-Lena \\ Date of birth: June 22, 1984 \\ Place of birth: Hannover, Germany \\ Nationality: German \\ 1994 - 2003 Grammar school, Marienberg Gymnasium in Neuss, Germany \\ 06/2003: 'Abitur' \\ 2003 - 2009 Student of mathematics, RWTH Aachen University, Germany \\ 01/2008 - 06/2008: University of York, United Kingdom \\ 06/2009: 'Diplom' in mathematics \\ $2009-2012$ Doctoral student at AICES Graduate School, \\ RWTH Aachen University, Germany
}

